\title{
Unidades de $\mathbb{Z} C_{p^{n}}$
}

\author{
Patrícia Massae Kitani
}

TESE APRESENTADA

$\mathrm{AO}$

Instituto De Matemática e Estatística

DA

Universidade de São Paulo

PARA

OBTENÇÃO DO TÍTULO

DE

Doutor EM CIÊNCIAS

Programa: Matemática

Orientador: Prof. Dr. Raul Antonio Ferraz

Durante o desenvolvimento deste trabalho o autor recebeu auxílio financeiro

da CAPES e do CNPq

São Paulo, março de 2012 



\section{Unidades de $\mathbb{Z} C_{p^{n}}$}

Este exemplar corresponde à redação final da tese devidamente corrigida e defendida por Patrícia Massae Kitani e aprovada pela Comissão Julgadora.

Banca Examinadora:

- Prof. Dr. Raul Antonio Ferraz (orientador) - IME-USP.

- Prof. Dr. Jairo Zacarias Gonçalves - IME-USP.

- Prof. Dr. Guilherme de La Rocque Leal - UFRJ.

- Profa. Dra. Marinês Guerreiro - UFV.

- Prof. Dr. Thierry Corrêa Petit Lobão - UFBA. 



\title{
Resumo
}

\author{
Unidades de $\mathbb{Z} C_{p^{n}}$
}

Seja $C_{p}$ um grupo cíclico de ordem $p$, onde $p$ é um número primo tal que $S=\{-1, \theta, 1+\theta$, $\left.1+\theta+\theta^{2}, \cdots, 1+\theta+\cdots+\theta^{\frac{p-3}{2}}\right\}$ gera o grupo das unidades de $\mathbb{Z}[\theta]$ e $\theta$ é uma raiz $p$-ésima primitiva da unidade sobre $\mathbb{Q}$. No artigo "Units of $\mathbb{Z} C_{p}$ ", Ferraz apresentou um modo simples de encontrar um conjunto de geradores independentes para o grupo das unidades do anel de grupo $\mathbb{Z} C_{p}$ sobre os inteiros. Nós estendemos este resultado para $\mathbb{Z} C_{p^{n}}$, considerando que um conjunto similar a $S$ gera o grupo das unidades de $\mathbb{Z}[\theta]$. Isto ocorre, por exemplo, quando $\phi\left(p^{n}\right) \leq 66$. Descrevemos o grupo das unidades de $\mathbb{Z} C_{p^{n}}$ como o produto $\pm \operatorname{ker}\left(\bar{\pi}_{1}\right) \times \operatorname{Im}\left(\bar{\pi}_{1}\right)$, onde $\bar{\pi}_{1}$ é um homomorfismo de grupos. Além disso, explicitamos as bases de $\operatorname{ker}\left(\bar{\pi}_{1}\right)$ e $\operatorname{Im}\left(\bar{\pi}_{1}\right)$.

Palavras-chave: anéis de grupo, grupos cíclicos, unidades de anéis de grupo sobre os inteiros, unidades simétricas normalizadas e unidades ciclotômicas. 



\section{Abstract}

\section{Units of $\mathbb{Z} C_{p^{n}}$}

Let $C_{p}$ be a cyclic group of order $p$, where $p$ is a prime integer such that $S=\{-1, \theta, 1+\theta$, $\left.1+\theta+\theta^{2}, \cdots, 1+\theta+\cdots+\theta^{\frac{p-3}{2}}\right\}$ generates the group of units of $\mathbb{Z}[\theta]$ and $\theta$ is a primitive $p^{\text {th }}$ root of 1 over $\mathbb{Q}$. In the article "Units of $\mathbb{Z} C_{p}$ ", Ferraz gave an easy way to find a set of multiplicatively independent generators of the group of units of the integral group ring $\mathbb{Z} C_{p}$. We extended this result for $\mathbb{Z} C_{p^{n}}$, provided that a set similar to $S$ generates the group of units of $\mathbb{Z}[\theta]$. This occurs, for example, when $\phi\left(p^{n}\right) \leq 66$. We described the group of units of $\mathbb{Z} C_{p^{n}}$ as the product $\pm \operatorname{ker}\left(\bar{\pi}_{1}\right) \times \operatorname{Im}\left(\bar{\pi}_{1}\right)$, where $\bar{\pi}_{1}$ is a group homomorphism. Moreover, we explicited a basis of $\operatorname{ker}\left(\bar{\pi}_{1}\right)$ and $\operatorname{Im}\left(\bar{\pi}_{1}\right)$.

Keywords: group rings, cyclic groups, units of integral group rings, normalized symmetric units and cyclotomic units. 



\section{Índice}

Introdução

1 Conceitos básicos $\quad 5$





1.3 Anel dos Inteiros Algébricos . . . . . . . . . . . . . . . . . 9

1.4 Anéis de Grupo . . . . . . . . . . . . . . . . . . . 13

2 Unidades de anéis de grupo modular $\quad 23$

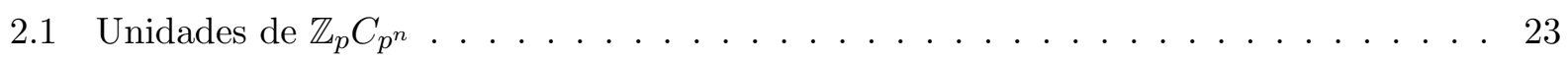

3 Unidades de $\mathbb{Z}[\theta] \quad 31$

3.1 Unidades Ciclotômicas . . . . . . . . . . . . . . . . . . . 31



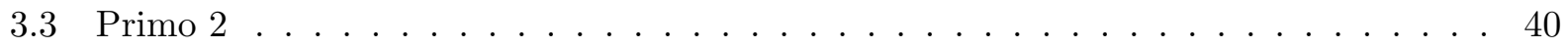

4 Unidades de $\mathbb{Z} C_{p^{n}} \quad 45$

4.1 Uma caracterização para $U_{1}\left(\mathbb{Z} C_{p^{n}}\right) \ldots \ldots \ldots \ldots \ldots \ldots$

4.2 Geradores Independentes para $\operatorname{ker}\left(\bar{\pi}_{1}\right) \ldots \ldots \ldots \ldots$

4.3 Validade da hipótese da Proposição $4.2 \ldots \ldots$. . . . . . . . . . . . . . 68

$\begin{array}{ll}\text { Considerações finais } & 71\end{array}$

$\begin{array}{ll}\text { Apêndice } & 73\end{array}$

$\begin{array}{lr}\text { Bibliografia } & 97\end{array}$ 



\section{Introdução}

Este trabalho consiste em procurar uma base para o grupo das unidades de certos anéis de grupo sobre os inteiros.

O estudo dos anéis de grupo teve início de uma forma implícita no artigo [11] de A. Cayley em 1854, mas explicitamente este assunto foi definido por T. Molien em 1897. Mais tarde E. Noether, R. Brauer e I. Schur contribuiram para o início do desenvolvimento da Teoria de Anéis de Grupo, estabelecendo uma relação entre a Teoria de Estrutura de Anéis e Álgebras e a Teoria de Representações de Grupos Finitos. Desde então, estes anéis têm sido objeto de pesquisa em áreas como Teoria de Grupos e Teoria dos Números. Particularmente, a Teoria de Números desempenha um papel importante no estudo de unidades de anéis de grupo sobre os inteiros.

Muitos pesquisadores tem se dedicado ao estudo do grupo das unidades de um anel de grupo. Citamos por exemplo R. Z. Aleev [1], [2], S. D. Berman [4], A. Bovdi [7], [8], [9], [10], R. Ferraz [14], [15], A. Giambruno, [16] J. Gonçalves [17], [18], [19], [20], G. Higman [22], E. Jespers [23], [24], [25], [26], M. Parmenter [34], [35], [36], D. S. Passman [18], C. Polcino [37], [38], [40], J. Ritter [41], [42], [43], S. Sehgal [45], [46], dentre outros.

Na verdade descrever o grupo das unidades $U(\mathbb{Z} G)$ do anel de grupo $\mathbb{Z} G$ sobre os inteiros, para um grupo finito $G$ é um problema clássico e difícil. A maioria das descrições de $U(\mathbb{Z} G)$ na literatura dão uma descrição explícita das unidades, ou a estrutura geral de $U(\mathbb{Z} G)$, ou um subgrupo de $U(\mathbb{Z} G)$ de índice finito. Tais resultados em sua maioria foram obtidos através de técnicas da Teoria de Representações e da Teoria Algébrica dos Números.

Neste trabalho estamos interessados em encontrar o conjunto de geradores independentes para $U\left(\mathbb{Z} C_{p^{n}}\right)$, onde $C_{p^{n}}$ denota o grupo cíclico de ordem $p^{n}$. Aqui, $p$ é um primo que deve satisfazer a seguinte condição: 


$$
S_{\theta}:=\left\{ \pm \theta, \mu_{t} \mid \mu_{t}=1+\theta+\theta^{2}+\cdots+\theta^{t-1}, 1<t<p^{n} / 2 \text { e }\left(t, p^{n}\right)=1\right\}
$$

gera $U(\mathbb{Z}[\theta])$, onde $\theta$ é uma raiz $p^{n}$-ésima primitiva da unidade.

O elemento $\mu_{t}$ é uma unidade no anel dos inteiros $\mathbb{Z}[\theta]$ e é conhecida como unidade ciclotômica. Em 1851, Kummer estabeleceu o seguinte resultado:

$$
S_{\theta} \text { gera } U(\mathbb{Z}[\theta]) \Longleftrightarrow h_{p^{n}}^{+}=1
$$

onde $h_{p^{n}}^{+}$é o número de classe de $\mathbb{Q}\left(\theta+\theta^{-1}\right)$. Assim, para determinar se $S_{\theta}$ gera $U(\mathbb{Z}[\theta])$ bastaria encontrar as potências de primos tais que $h_{p^{n}}^{+}=1$. No entanto, encontrar estes números tem se mostrado um problema muito difícil. Até onde sabemos, poucos casos foram obtidos, como por exemplo para $p$ tal que $\phi\left(p^{n}\right) \leq 66$.

Neste trabalho, tomamos $p$ satisfazendo a condição $(*)$, pois baseamos nossa pesquisa no artigo de Ferraz [15], onde os primos considerados neste artigo são os compreendidos entre os números 5 e 70. Conseguimos estender os resultados obtidos por Ferraz para as potências $p^{n}$ tais que $\phi\left(p^{n}\right) \leq 66$.

A tese está apresentada da seguinte forma:

No primeiro capítulo, enunciamos os conceitos básicos que necessitamos na tese. Citamos brevemente definições e resultados sobre grupos, polinômios ciclotômicos, anel dos inteiros algébricos e anéis de grupo. Como estamos interessados em descrever uma base para as unidades de $\mathbb{Z} C_{p^{n}}$, citamos várias unidades clássicas neste capítulo, mas uma unidade em especial foi de grande relevância neste desenvolvimento: a unidade de Hoechsmann.

Definição 0.1. Sejam $C_{n}$ um grupo cíclico de ordem $n$ e $C_{n}=\langle g\rangle$. O elemento $u_{b c}$ abaixo é uma unidade de $\mathbb{Z} C_{n}$ chamada unidade de Hoechsmann:

$$
u_{b c}=\left(1+g^{b}+g^{2 b}+\cdots+g^{b(a-1)}\right)\left(1+g^{c}+g^{2 c}+\cdots+g^{c(b-1)}\right)+\frac{1-a b}{n} \widehat{g}
$$

onde $a, b, c \in \mathbb{Z}$ tais que $\operatorname{mdc}(b, n)=\operatorname{mdc}(c, n)=1$ e $a b \equiv 1(\bmod n)$.

O segundo capítulo é sucinto e nele descrevemos as unidades do anel de grupo modular, para o grupo cíclico de ordem $p^{n}$. Este capítulo basicamente se deve ao fato de utilizarmos o seguinte diagrama comutativo:

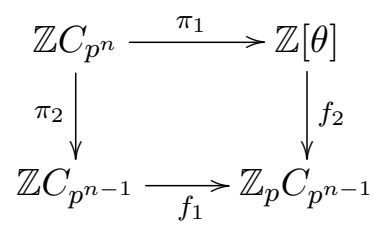


Se $C_{p^{n}}=\langle g\rangle$ e $C_{p^{n-1}}=\langle h\rangle$, então os homomorfismos do diagrama estão definidos do seguinte modo: $\pi_{1}(g)=\theta, \pi_{2}(g)=h, f_{1}(h)=h$ e $f_{2}(\theta)=h$.

No terceiro capítulo, iniciamos o trabalho, estabelecendo resultados a respeito das unidades do anel $\mathbb{Z}[\theta]$. Todos os resultados neste capítulos são análogos aos do artigo [15], com algumas hipótese modificadas. Em geral, a hipótese adicional é de que o conjunto $S_{\theta}$, mencionado em $(*)$, gera $U(\mathbb{Z}[\theta])$, que $t$ é um inteiro tal que $U\left(\mathbb{Z}_{p^{n}}\right)=\langle\bar{t}\rangle$, no caso de $p$ ser ímpar, e $t=3$, no caso $p=2$.

Por fim, no quarto capítulo descrevemos as unidades que procuramos. Provamos o isomorfismo

$$
U_{1}\left(\mathbb{Z} C_{p^{n}}\right) \simeq \operatorname{ker}\left(\bar{\pi}_{1}\right) \times\left\langle\operatorname{Im}\left(\bar{\pi}_{1}\right)\right\rangle
$$

onde $\bar{\pi}_{1}$ é o homomorfismo de grupos proveniente do homomorfismo de anéis $\pi_{1}: \mathbb{Z} C_{p^{n}} \rightarrow \mathbb{Z}[\theta]$. Os geradores independentes para $\operatorname{Im}\left(\bar{\pi}_{1}\right)$ foram encontradas de modo geral, no entanto, não conseguimos um método geral para encontrar os geradores independentes do núcleo de $\bar{\pi}_{1}$. Mostramos caso a caso para os primos que satistazem a nossa condição $(*)$. 



Iniciamos este capítulo com conceitos básicos que foram utilizados durante o trabalho. Como o nosso foco é unidades de anéis de grupo, é necessário uma fundamentação nas Teorias de Grupo ou Anel, uma vez que todos os resultados em anéis de grupo estão relacionados com os resultados provenientes de ambas as teorias.

Alguns resultados serão assumidos sem as provas, mas neste caso as devidas referências bibliográficas serão indicadas e as notações apresentadas neste capítulo serão, em geral, as mesmas das utilizadas no decorrer do trabalho.

As referências utilizadas neste capítulo foram as seguintes: [27], [28], [29],[31], [32], [33], [39], $[45]$ e $[48]$.

\subsection{Grupos}

Nesta seção citaremos alguns resultados bastante conhecidos da Teoria de Grupos. Para a operação de um grupo abeliano, utilizaremos a notação multiplicativa, apesar de muitas vezes, na literatura, ser utilizado a notação aditiva. Denotaremos, de modo geral, um grupo por $G$, a menos de menção em contrário.

Definição 1.1. Um grupo abeliano $G$ é finitamente gerado se existirem $g_{1}, g_{2}, \cdots, g_{m}$ em $G$ tais que

$$
g=g_{1}^{n_{1}} \cdot g_{2}^{n_{2}} \cdots g_{m}^{n_{m}} \quad n_{j} \in \mathbb{Z}
$$

para todo $g \in G$. 
Denotamos por $G=<g_{1}, g_{2}, \cdots, g_{m}>$ o grupo $G$ gerado por $g_{1}, g_{2}, \cdots, g_{m}$.

Definição 1.2. Dizemos que $g_{1}, g_{2}, \cdots, g_{m} \in G$ são multiplicativamente independentes sobre $\mathbb{Z}$ se a equação

$$
g_{1}^{n_{1}} \cdot g_{2}^{n_{2}} \cdots \cdot g_{m}^{n_{m}}=1
$$

com $n_{j} \in \mathbb{Z}$ implica $n_{j}=0$, para todo $1 \leq j \leq m$. Um conjunto de elementos multiplicativamente independentes e que gera $G$, é chamado de base de $G$.

Definição 1.3. Um grupo abeliano que possuir uma base com $n$ elementos é chamado grupo abeliano livre de posto $n$.

Sabemos que se $G$ é um grupo abeliano que possui base, então duas bases quaisquer de $G$ possuem o mesmo número de elementos.

Utilizamos o teorema a seguir para encontrar o índice de um subgrupo no qual estamos interessados. Em nosso caso, o índice nos auxilia a indicar se um subgrupo é ou não gerado por um certo conjunto.

Teorema 1.1. Se $G$ é um grupo abeliano livre de posto $n$ e $H$ um subgrupo de $G$, então $G / H$ é finito se, e somente se, $H$ tiver posto $n$. Nesse caso, se $\left\{x_{1}, \cdots, x_{n}\right\}$ e $\left\{y_{1}, \cdots, y_{n}\right\}$ são bases de $G$ e $H$, respectivamente, com

$$
y_{i}=\prod_{j}^{n} x_{j}^{a_{i j}},
$$

então

$$
|G: H|=\left|\operatorname{det}\left(a_{i j}\right)\right|
$$

Um outro resultado bastante conhecido é o grupo dos restos módulo $n$, geralmente denotado por $\mathbb{Z} / n \mathbb{Z}$ ou $\mathbb{Z}_{n}$. O que nos interessa aqui é o grupo multiplicativo das unidades de $\mathbb{Z}_{n}$, denotado por $U\left(\mathbb{Z}_{n}\right)$. Mais especificamente o que queremos são os casos em que este grupo tem a estrutura de um grupo cíclico. Para isto, temos o seguinte teorema:

Teorema 1.2. O grupo $U\left(\mathbb{Z}_{n}\right)$ é cíclico se, e somente se, $n=1,2,4, p^{t}$ ou $2 p^{t}$, onde p é um número primo impar.

Para $n=2^{t}$, com $t \geq 3$, o grupo $\mathbb{Z}_{n}$ não é cíclico. Neste caso, temos uma outra estrutura que daremos logo a seguir. 
Definição 1.4. Um inteiro $t$ é chamado de raiz primitiva módulo $n$ se $\bar{t}$ for um gerador do grupo $U\left(\mathbb{Z}_{n}\right)$.

Definição 1.5. Sejam $m$ um número inteiro positivo e z um inteiro qualquer tais que $m d c(m, z)=$ 1. Seja $h$ o menor número inteiro positivo tal que $z^{h} \equiv 1(\bmod m)$. Dizemos que a ordem de $z$ módulo $m$ é $h$, ou que z tem ordem $h(\bmod m)$.

Denotaremos por $C_{n}$ o grupo cíclico de ordem $n$.

Teorema 1.3. Se $t \geq 3$, então $U\left(\mathbb{Z}_{2^{t}}\right) \cong C_{2} \times C_{2^{t-2}}$. Além disso, a ordem de 5 módulo $2^{t}$ é $2^{t-2}$ e os números $\pm 5, \pm 5^{2}, \pm 5^{3} \cdots, \pm 5^{2^{t-2}}$ formam um conjunto de representantes de classes de $U\left(\mathbb{Z}_{2^{t}}\right)$. Se $x$ for ímpar, então existem $i, j$ tais que $x \equiv(-1)^{i} 5^{j}\left(\bmod 2^{t}\right)$. Os valores de $i$ e $j$ são univocamente determinados $(\bmod 2)$ e $\left(\bmod 2^{t}\right)$ respectivamente.

No Teorema 1.3, podemos também tomar como representantes das classes de $U\left(\mathbb{Z}_{2^{t}}\right)$ os números $\pm 3, \pm 3^{2}, \pm 3^{3} \cdots, \pm 3^{2^{t-2}}$.

Assim, no Capítulo 3 usaremos a seguinte caracterização:

$$
U\left(\mathbb{Z}_{2^{t}}\right) \cong C_{2} \times C_{2^{t-2}} \cong\left\langle 2^{t}-1\right\rangle \times\langle 3\rangle
$$

\subsection{Polinômio Ciclotômico}

Vamos relembrar brevemente alguns conceitos de raízes da unidade e polinômios ciclotômicos. Voltaremos a utilizar este polinômio mais adiante para definirmos um importante homomorfismo neste trabalho.

Definição 1.6. Seja $\mathbb{L}$ um corpo e $n \in \mathbb{Z}, n \geq 1$. Um elemento $\zeta \in \mathbb{L}$ tal que $\zeta^{n}=1$ é chamado uma raiz $n$-ésima da unidade.

Seja $n>1$ um inteiro não divisível pela característica do corpo $\mathbb{L}$. O polinômio $x^{n}-1 \in \mathbb{L}[x]$ é separável, pois sua derivada é $n x^{n-1} \neq 0$, e a única raiz da derivada é 0 , logo todas as raízes de $x^{n}-1$ são simples. Consequentemente, no fecho algébrico de $\mathbb{L}$, o polinômio $x^{n}-1$ possui $n$ raízes distintas, que são as raízes $n$-ésimas da unidade. Estas raízes formam um grupo multiplicativo e como todo grupo multiplicativo finito de um corpo é cíclico, segue que o grupo das raízes $n$-ésimas da unidade é cíclico. Um gerador para este grupo é chamado raiz $n$-ésima primitiva da unidade e denotaremos de agora em diante esta raiz por $\zeta_{n}$. 
Vamos analisar a fatoração do polinômio $x^{n}-1 \in \mathbb{Q}[x]$. Na realidade, os resultados a seguir valem para um corpo qualquer tal que a característica não divida $n$, no entanto restringiremos para o caso do conjunto dos números dos racionais $\mathbb{Q}$.

Temos $x^{n}-1=\prod_{\zeta}(x-\zeta)$, onde $\zeta$ são todas as raízes $n$-ésimas da unidade. Agora reunindo todos os termos de $x^{n}-1=\prod_{\zeta}(x-\zeta)$ tais que as raízes da unidade possuem a mesma ordem, obtemos um polinômio que denotaremos por

$$
\Phi_{d}(x)=\prod_{\text {ordem } \zeta=d}(x-\zeta)
$$

Logo

$$
x^{n}-1=\prod_{d \mid n} \Phi_{d}(x) .
$$

Decorre disto que $\Phi_{n}(x)=\frac{x^{n}-1}{\prod_{d} \Phi_{d}(x)}$, para $0<d<n$.

Definição 1.7. Chamamos $\Phi_{n}(x)$ de n-ésimo polinômio ciclotômico.

Proposição 1.4. Seja $p$ um número primo e $r \geq 1$ um inteiro. Então

$$
\Phi_{p}(x)=\frac{x^{p}-1}{x-1}=x^{p-1}+x^{p-2}+\cdots+x^{2}+x+1
$$

$e$

$$
\Phi_{p^{r}}(x)=\Phi_{p}\left(x^{p^{r-1}}\right)
$$

\section{Prova:}

Provemos esta última fórmula:

$$
\Phi_{p^{r}}(x)=\frac{x^{p^{r}}-1}{\prod_{d \mid p^{r}} \Phi_{d}(x)}=\frac{\left(x^{p^{r-1}}-1\right)\left(x^{(p-1) p^{r-1}}+x^{(p-2) p^{r-1}}+\cdots+x^{p^{r-1}}+1\right)}{\prod_{d \mid p^{r}} \Phi_{d}(x)}, \operatorname{com} d<p^{r}
$$

$\operatorname{Mas} \prod_{d \mid p^{r}} \Phi_{d}(x)=x^{p^{r-1}}-1$, para $d<p^{r}, \log \mathrm{O}$

$$
\Phi_{p^{r}}(x)=\left(x^{(p-1) p^{r-1}}+x^{(p-2) p^{r-1}}+\cdots+x^{p^{r-1}}+1\right)=\Phi_{p}\left(x^{r-1}\right) . \mathbf{x}
$$


Lema 1.5. Sejam $E \subseteq F$ uma extensão de corpos e $\alpha \in E$ um elemento algébrico sobre F. Se $\alpha$ é raiz de um polinômio mônico irredutivel $f(x)$ sobre $F$, então $f(x)$ é o polinômio minimal de $\alpha$.

\section{Prova:}

Seja $p(x)$ o polinômio minimal de $\alpha$ sobre $F$, logo mônico. Como $\alpha$ é raiz de $f(x)$ e $p(x)$ é polinômio minimal de $\alpha$ segue que $p(x)$ divide $f(x)$, ou seja, $f(x)=p(x) h(x)$, com $h(x) \in F[x]$. Como $f(x)$ é irredutível sobre $F$ temos $f(x)=b \cdot p(x), b \in F$ e como $f(x)$ e $p(x)$ são mônicos, devemos ter $b=1$, isto é, $f(x)=p(x)$. Portanto, $f(x)$ é o polinômio minimal de $\alpha$. ¿z

Lema 1.6. Seja $n \geq 1$ um número inteiro. Então o n-ésimo polinômio ciclotômico é irredutível sobre $\mathbb{Z}$.

Este resultado se deve a Gauss, a prova não é trivial e pode ser encontrada em [31].

Como consequência destes lemas temos que $\Phi_{n}(x)$ é um polinômio minimal de $\zeta_{n}$ sobre $\mathbb{Z}$.

\subsection{Anel dos Inteiros Algébricos}

Veremos agora alguns conceitos sobre os anéis dos inteiros algébricos de $\mathbb{Q}\left(\zeta_{n}\right)$. O grupo das unidades de $\mathbb{Z}\left[\zeta_{n}\right]$ tem a sua importância em nosso problema, pois a partir deste grupo obtemos alguns resultados relevantes neste trabalho. Na verdade, este resultado está intimamente ligado com os resultados sobre as unidades de $\mathbb{Z}\left[\zeta_{n}\right]$.

Definição 1.8. O corpo dos números algébricos ou corpo numérico é uma extensão finita do corpo $\mathbb{Q}$ dos números racionais. Se $K$ é um corpo de números algébricos, o fecho integral de $\mathbb{Z}$ em $K$ é também chamado de anel dos inteiros algébricos de $K$.

Definição 1.9. Um corpo ciclotômico é um corpo gerado por uma raiz n-ésima da unidade.

O corpo ciclotômico $\mathbb{Q}\left(\zeta_{n}\right)$ é um corpo numérico obtido pela adjunção de $\zeta_{n}$ o corpo dos racionais $\mathbb{Q}$.

Teorema 1.7. $\mathbb{Z}\left[\zeta_{n}\right]$ é o anel dos inteiros algébricos no corpo ciclotômico $\mathbb{Q}\left(\zeta_{n}\right)$.

Temos ainda $\left|\mathbb{Z}\left[\zeta_{n}\right]: \mathbb{Z}\right|=\left|\mathbb{Q}\left(\zeta_{n}\right): \mathbb{Q}\right|=\phi(n)$, onde $\phi$ é a função de Euler.

Agora seja $K=\mathbb{Q}\left(\zeta_{n}\right)$ e $f(x) \in \mathbb{Q}[X]$ o polinômio minimal de $\zeta_{n}$. Denote por $\alpha_{1}, \alpha_{2}, \cdots, \alpha_{r_{1}}$ $\left(\left\{\beta_{1}, \bar{\beta}_{1}\right\}, \cdots\left\{\beta_{r_{2}}, \bar{\beta}_{r_{2}}\right\}\right.$, respectivamente) todas as raízes reais (todos os pares das raízes complexas 
conjugadas, respectivamente) de $f(x)$. Então $f(x)$ decompõe-se em $r_{1}+r_{2}$ fatores irredutíveis sobre os reais $\mathbb{R}$ e $\left|\mathbb{Q}\left(\zeta_{n}\right): \mathbb{Q}\right|=r_{1}+2 r_{2}$. O par $\left(r_{1}, r_{2}\right)$ é chamado assinatura de $K$.

O teorema seguinte determina o posto do grupo das unidades do anel dos inteiros algébricos de um corpo numérico. Este resultado foi estabelecido por Dirichlet, em 1840, e uma versão mais geral foi provada por Chevalley e Hasse, um século depois. Uma prova do teorema pode ser encontrada em [28] na página 24.

Teorema 1.8 (Teorema 2.8.2, [39] - Unidades de Dirichlet). Sejam $R$ o anel dos inteiros de um corpo de números algébricos $K$ e $\left(r_{1}, r_{2}\right)$ a assinatura de $K$. Então o grupo das unidades de $R$ é um produto direto de um grupo cíclico de ordem finita e um grupo abeliano de posto $r_{1}+r_{2}-1$.

Observação 1.1. Seja $R$ um anel. Denotamos por $U(R)$ o grupo multiplicativo das unidades de $R$, isto é, $U(R)=\{x \in R: \exists y \in$ Rtal que $x y=y x=1\}$

Corolário 1.9. Seja $\zeta_{n}$ uma raiz n-ésima primitiva da unidade sobre $\mathbb{Q}$, onde $n>2$. Então

$$
U\left(\mathbb{Z}\left[\zeta_{n}\right]\right)= \begin{cases}\left\langle\zeta_{n}\right\rangle \times A & \text { se n é par } \\ \left\langle-\zeta_{n}\right\rangle \times A & \text { se n é ímpar }\end{cases}
$$

onde A é um grupo abeliano livre de posto $\frac{\phi(n)}{2}-1$.

\section{Prova:}

Pelo Teorema 1.7 sabemos que $\mathbb{Z}\left[\zeta_{n}\right]$ é o anel dos inteiros de $\mathbb{Q}\left(\zeta_{n}\right)$ e $\phi(n)$ é o grau de $\mathbb{Q}\left(\zeta_{n}\right)$ sobre $\mathbb{Q}$. Como $n>2$, nenhuma raiz $n$-ésima primitiva de 1 é real em $\mathbb{Q}\left(\zeta_{n}\right)$. Logo $r_{1}=0, r_{2}=\frac{\phi(n)}{2}$ e, portanto, $r_{1}+r_{2}-1=\frac{\phi(n)}{2}-1$. Além disso, o subgrupo de torção do grupo multiplicativo de $\mathbb{Q}\left(\zeta_{n}\right)$ é $\left\langle\zeta_{n}\right\rangle$, se $n$ é par, e $\left\langle-\zeta_{n}\right\rangle$, se $n$ é ímpar. ¿̀

Como consequência deste corolário, exitem $r=\frac{1}{2} \phi(n)-1$ unidades $\mu_{1}, \mu_{2}, \cdots, \mu_{r}$ de $\mathbb{Z}\left[\zeta_{n}\right]$ tais que cada unidade $u \in \mathbb{Z}\left[\zeta_{n}\right]$ pode ser expressa unicamente da forma $u=\delta \mu_{1}^{n_{1}} \mu_{2}^{n_{2}} \cdots \mu_{r}^{n_{r}}$, com $n_{i} \in \mathbb{Z}$ e $\delta \in\left\langle \pm \zeta_{n}\right\rangle$, o subgrupo de torção de $U\left(\mathbb{Z}\left[\zeta_{n}\right]\right)$.

Definição 1.10. O conjunto $\left\{\mu_{1}, \mu_{2}, \cdots, \mu_{r}\right\}$ acima é chamado de sistema fundamental das unidades de $U\left(\mathbb{Z}\left[\zeta_{n}\right]\right)$.

Proposição 1.10. Suponha $r$ e s inteiros tais que $(n, r s)=1$. Então $\frac{\zeta_{n}^{r}-1}{\zeta_{n}^{s}-1}$ é uma unidade de $\mathbb{Z}\left[\zeta_{n}\right]$. 


\section{Prova:}

Como $m d c(n, r s)=1$, temos $m d c(n, r)=m d c(n, s)=1$. Portanto,

$$
r \equiv s t(\bmod n) \text { e } s \equiv r l(\bmod n)
$$

para $\operatorname{algum} t, l \in \mathbb{Z}$. Então

$$
u=\frac{\zeta_{n}^{r}-1}{\zeta_{n}^{s}-1}=\frac{\zeta_{n}^{s t}-1}{\zeta_{n}^{s}-1}=1+\zeta_{n}+\cdots+\zeta_{n}^{s(t-1)} \in \mathbb{Z}\left[\zeta_{n}\right]
$$

e

$$
u^{-1}=\frac{\zeta_{n}^{s}-1}{\zeta_{n}^{r}-1}=\frac{\zeta_{n}^{r l}-1}{\zeta_{n}^{r}-1}=1+\zeta_{n}+\cdots+\zeta_{n}^{r(l-1)} \in \mathbb{Z}\left[\zeta_{n}\right]
$$

e isto completa a prova. is

As unidades $\mu_{a}=\frac{\zeta_{n}^{a}-1}{\zeta_{n}-1} \operatorname{com} m d c(a, n)=1$ são chamadas unidades ciclotômicas e são importantes neste trabalho. De agora em diante, $\mu_{a}$ sempre denotará uma unidade ciclotômica.

É possível construir $\frac{1}{2} \phi(n)-1$ unidades independentes de $U\left(\mathbb{Z}\left[\zeta_{n}\right]\right)$ no caso em que $n$ é uma potência de primos. Para tanto, devemos estabelecer uma relação entre $U\left(\mathbb{Z}\left[\zeta_{n}\right]\right)$ e o grupo das unidades do anel dos inteiros do subcorpo real maximal $\mathbb{Q}\left(\zeta_{n}+\zeta_{n}^{-1}\right)$.

Observe que se $\alpha \in \mathbb{Q}\left(\zeta_{n}\right) \subseteq \mathbb{C}$, então o complexo conjugado $\bar{\alpha}$ também pertence a $\mathbb{Q}\left(\zeta_{n}\right)$ pois $\bar{\zeta}_{n}=\zeta_{n}^{-1}$. Portanto, a conjugação complexa induz um automorfismo do corpo $\mathbb{Q}\left(\zeta_{n}\right)$.

Teorema 1.11. Seja $C=\left\{\alpha \in \mathbb{Q}\left(\zeta_{n}\right): \bar{\alpha}=\alpha\right\}$ o conjunto dos elementos de $\mathbb{Q}\left(\zeta_{n}\right)$ fixos pela conjugação complexa. Então

$$
\mathbb{Q}\left(\zeta_{n}+\zeta_{n}^{-1}\right)=\left\{\alpha \in \mathbb{Q}\left(\zeta_{n}\right): \bar{\alpha}=\alpha\right\}
$$

\section{$\mathbb{Q}\left(\zeta_{n}+\zeta_{n}^{-1}\right)$ é chamado subcorpo real maximal de $\mathbb{Q}\left(\zeta_{n}\right)$.}

Determinar o sistema fundamental das unidades de $U\left(\mathbb{Z}\left[\zeta_{n}\right]\right)$ tem sido um problema difícil e até onde sabemos, foram determinados somente para alguns valores de $n$, como veremos mais a diante. Na verdade, a dificuldade tem sido em encontrar o número de classe de $\mathbb{Q}\left(\zeta_{n}+\zeta_{n}^{-1}\right)$, geralmente denotada por $h^{+}$.

\section{Lema 1.12.}

(i) $\mathbb{Q}\left(\zeta_{n}+\zeta_{n}^{-1}\right)=\mathbb{Q}\left(\zeta_{n}\right) \cap \mathbb{R}=\mathbb{Q}(\cos (2 \pi / n))$.

(ii) A extensão $\mathbb{Q}\left(\zeta_{n}\right) / \mathbb{Q}\left(\zeta_{n}+\zeta_{n}^{-1}\right)$ é de grau 2 . 
(iii) $\mathbb{Z}\left[\zeta_{n}+\zeta_{n}^{-1}\right]$ é o anel dos inteiros de $\mathbb{Q}\left(\zeta_{n}+\zeta_{n}^{-1}\right)$.

\section{Prova:}

Observe que $(i)$ segue de $(i i)$, que por sua vez é consequência do fato de $\zeta_{n}$ ser uma raiz de $x^{2}-\left(\zeta_{n}+\zeta_{n}^{-1}\right) x+1$.

Para a prova de $($ iii $)$, seja $R$ o anel dos inteiros de $\mathbb{Q}\left(\zeta_{n}+\zeta_{n}^{-1}\right)$. Claro que $\mathbb{Z}\left[\zeta_{n}+\zeta_{n}^{-1}\right] \subseteq R$. Para provar a outra inclusão, suponha $\alpha=a_{0}+a_{1}\left(\zeta_{n}+\zeta_{n}^{-1}\right)+\cdots+a_{s}\left(\zeta_{n}+\zeta_{n}^{-1}\right)^{s} \in R$, com $s \leq \frac{1}{2} \phi(n)-1$ e $a_{i} \in \mathbb{Q}$. Removendo os termos $a_{i} \in \mathbb{Z}$, podemos assumir $a_{s} \notin \mathbb{Z}$. Multiplicando $\alpha$ por $\zeta_{n}^{s}$ e expandindo o resultado como um polinômio em $\zeta_{n}$, obtemos $\zeta_{n}^{s} \alpha=a_{s}+\cdots+a_{s} \zeta_{n}^{2 s}$ que é um inteiro algébrico em $\mathbb{Q}\left(\zeta_{n}\right)$ e, portanto, está em $\mathbb{Z}\left[\zeta_{n}\right]$. Mas como $2 s \leq \phi(n)-2 \leq \phi(n)-1$, segue que $\left\{1, \zeta_{n}, \cdots \zeta_{n}{ }^{2 s}\right\}$ é um subconjunto de uma $\mathbb{Z}$-base para o anel $\mathbb{Z}\left[\zeta_{n}\right]$. Logo $a_{s} \in \mathbb{Z}$, o que é um absurdo. E isto completa a prova de (iii). is

Pelo lema anterior temos $U\left(\mathbb{Z}\left[\zeta_{n}+\zeta_{n}^{-1}\right]\right)=\mathbb{R} \cap U\left(\mathbb{Z}\left[\zeta_{n}\right]\right)$.

Antes de prosseguir com o principal resultado desta seção, estabelecemos algumas notações:

$$
\begin{aligned}
\mu_{a} & =\left(1-\zeta_{n}{ }^{a}\right) /\left(1-\zeta_{n}\right), \operatorname{mdc}(n, a)=1 \\
\mu_{a}{ }^{+} & =\zeta_{n}{ }^{(1-a) / 2} \mu_{a} \\
B_{n} & =\left\langle \pm 1, \zeta_{n}, \mu_{a} \mid 1<a<n / 2, \operatorname{mdc}(a, n)=1\right\rangle \\
B_{n}^{+} & =\left\langle \pm 1, \zeta_{n}, \mu_{a}^{+} \mid 1<a<n / 2, \operatorname{mdc}(a, n)=1\right\rangle
\end{aligned}
$$

Teorema 1.13 (Teorema 3.3.3, [28] - Kummer 1851). Seja $n=p^{n}$ uma potência de um número primo p. Então:

(i) As unidades ciclotômicas $\mu_{a}, 1<a<n / 2$, com $m d c(a, n)=1$, são independentes e geram um subgrupo de indice finito em $U\left(\mathbb{Z}\left[\zeta_{n}\right]\right)$.

(ii) $\left|U\left(\mathbb{Z}\left[\zeta_{n}\right]\right): B_{n}\right|=\left|U\left(\mathbb{Z}\left[\zeta_{n}+\zeta_{n}^{-1}\right]\right): B_{n}^{+}\right|=h_{n}^{+}$.

Não é verdade que, para qualquer número inteiro $n$, o conjunto das unidades ciclotômicas $\mu_{a}$ é independente. Este fato foi provado por Ramachandra em 1966, para o caso $n=55$, no artigo "On the units of cyclotomic fields".

Corolário 1.14. Se n é uma potência de um número primo, então as seguintes condições são equivalentes:

(i) $B_{n}$ é um sistema fundamental das unidades de $U\left(\mathbb{Z}\left[\zeta_{n}\right]\right)$;

(ii) $B_{n}^{+}$é um sistema fundamental das unidades de $U\left(\mathbb{Z}\left[\zeta_{n}+\zeta_{n}^{-1}\right]\right)$; 
(iii) $h_{n}^{+}=1$.

Até onde sabemos, somente para poucos casos de $n$ tem-se $h_{n}^{+}=1$.

Há cerca de 2 séculos atrás, Gauss estabeleceu a seguinte conjectura:

"Existem infinitos corpos quadráticos reais cujo número de classe vale 1."

Esta conjectura ainda permanece aberta e baseada nela, Weber considerou os seguintes problemas que também continuam sem soluções:

"Existem infinitos corpos numéricos com número de classe 1."

$$
\text { " } h^{+}=\text {o número de classes de } \mathbb{Q}\left(\cos \left(2 \pi / 2^{m}\right)\right)=1 . "
$$

Sabemos que se $n$ é uma potência de primo tal que $\phi(n) \leq 66$, então $h_{n}^{+}=1$ (ver [48]). Com esta informação e o corolário anterior segue imediatamente o seguinte resultado:

Corolário 1.15. Seja $n$ uma potência de um número primo com $\phi(n) \leq 66$. Então $B_{n}$ é um sistema fundamental das unidades de $U\left(\mathbb{Z}\left[\zeta_{n}\right]\right)$.

É claro que se a segunda conjectura de Weber acima fosse verdadeira, $B_{2^{m}}$ também seria um sistema fundamental de $U\left(\mathbb{Z}\left[\zeta_{2^{m}}\right]\right)$.

Este último corolário foi o ponto de partida para este trabalho. Em [15], Ferraz verificou para os primos entre 5 e 67 . No entanto, percebendo que este conjunto poderia se estender para potências de primos nas condições do corolário acima, resolvemos verificar se os resultados do artigo poderiam ser válidos também para este caso. Veremos que sim, com algumas modificações.

\subsection{Anéis de Grupo}

Aqui citaremos alguns dos resultados básicos voltados para o grupo das unidades dos anéis de grupo. Estamos interessados em encontrar um conjunto de geradores independentes das unidades do anel de grupo sobre os inteiros, por isso os principais resultados nesta seção são sobre $U(\mathbb{Z} G)$.

Definição 1.11. Seja $G$ um grupo e seja $R$ um anel com unidade. Um anel de grupo representado por $R G$ é o conjunto de todas as somas formais do tipo $\sum_{g \in G} a_{g} g$, onde $a_{g} \in R$ são quase todos nulos, 
ou seja, apenas um número finito de elementos $a_{g}$ são distintos de zero, munido das seguintes operações:

(i) $\sum_{g \in G} a_{g} g+\sum_{g \in G} b_{g} g:=\sum_{g \in G}\left(a_{g}+b_{g}\right) g$;

(ii) $\left(\sum_{g \in G} a_{g} g\right) \cdot\left(\sum_{h \in G} b_{h} h\right):=\left(\sum_{g \in G} \sum_{h \in G}\left(a_{g} b_{h}\right) g h\right)$.

É fácil verificar que $(R G,+, \cdot)$ é um anel.

Podemos também definir a multiplicação de elementos de $R G$ por elementos do anel $R$ como segue:

(iii) $\lambda \cdot\left(\sum_{g \in G} a_{g} g\right):=\sum_{g \in G}\left(\lambda a_{g}\right) g$.

Desta forma, $R G$ admite uma estrutura de $R$-módulo.

E se $R$ for um anel comutativo, então $R G$ é uma álgebra sobre $R$, chamada de álgebra de grupo de $G$ sobre $R$.

A partir de agora $R$ denotará um anel e $G$ um grupo.

Definição 1.12. Sejam $R G$ um anel de grupo. O conjunto $\{x \in R G:(\exists y \in R G) x y=1=y x\}$ é chamado conjunto das unidades de $R G$ e será denotado por $U(R G)$.

Vejamos alguns exemplos de unidades de anel de grupo $\mathbb{R} G$, dos quais alguns são antigos e outros elementares.

Exemplo 1.1 (Unidades Triviais). Um elemento da forma $r g$, onde $r \in U(R)$ e $g \in G$ tem inverso $r^{-1} g^{-1}$. Elementos desta forma são chamados unidades triviais de $R G$.

Exemplo 1.2 (Unidades Unipotentes). Seja $\eta \in R$ tal que $\eta^{2}=0$. Então $(1+\eta)(1-\eta)=1$, ou seja, $(1+\eta)$ e $(1-\eta)$ são unidades de $R$. Do mesmo modo, se $\eta \in R$ tal que $\eta^{k}=0$, para algum inteiro positivo $k$, então temos

$$
\begin{gathered}
(1-\eta)\left(1+\eta+\eta^{2}+\cdots+\eta^{k-1}\right)=1 \\
(1+\eta)\left(1-\eta+\eta^{2} \cdots \pm \eta^{k-1}\right)=1
\end{gathered}
$$

Logo $1 \pm \eta$ são unidades de $R$, chamadas de unidades unipotentes de $R$. 
Exemplo 1.3 (Unidades Bicíclicas). Sejam $a \in G$ um elemento de ordem finita $n$ em $G$ e $b$ um elemento qualquer de $G$. Então $(a-1)\left(1+a+a^{2}+\cdots+a^{n-1}\right)=0$. O elemento $\mu_{a, b}=1+(a-1) b \widehat{a}$, com $\widehat{a}=1+a+a^{2}+\cdots+a^{n-1}$, é uma unidade de $\mathbb{Z} G$ chamada unidade bicíclica, onde a inversa é o elemento $\mu_{a, b}^{-1}=1-(a-1) b \widehat{a}$.

Observe que se $b a=a b$, então $\mu_{a, b}=1$ pois neste caso $(a-1) b \widehat{a}=(a-1) \widehat{a} b=(\widehat{a}-\widehat{a}) b=0$. De um modo mais geral, se $b$ não pertence ao normalizador de $\langle a\rangle$, então $\mu_{a, b} \neq 1$.

Exemplo 1.4 (Unidade Cíclica de Bass). Seja g um elemento de ordem n em G. Uma unidade cíclica de Bass é um elemento de $\mathbb{Z} G$ da forma

$$
\mu_{i}=\left(1+g+\cdots+g^{i-1}\right)^{\phi(n)}+\frac{1-i^{\phi(n)}}{n} \widehat{g}
$$

onde $i \in \mathbb{Z}$ é tal que $1<i<n-1, m d c(n, i)=1, \widehat{g}=1+g+g^{2}+\cdots+g^{n-1}$ e $\phi$ a função de Euler.

Exemplo 1.5 (Unidades de Hoechsmann). Seja $C_{n}=\langle g\rangle$ um grupo cíclico de ordem $n$. O seguinte elemento é uma unidade de $\mathbb{Z} C_{n}$ chamada unidade de Hoechsmann:

$$
u_{b c}=\left(1+g^{b}+g^{2 b}+\cdots+g^{b(a-1)}\right)\left(1+g^{c}+g^{2 c}+\cdots+g^{c(b-1)}\right)+\frac{1-a b}{n} \widehat{g}
$$

onde $a, b, c \in \mathbb{Z}$ são tais que $\operatorname{mdc}(b, n)=\operatorname{mdc}(c, n)=1$ e $a b \equiv 1(\bmod n)$

A unidade de Hoechsmann, em particular, é muito importante para os resultados desta tese e por isso verificaremos que $u_{b c}$ definida em $(* *)$ é uma unidade de $\mathbb{Z} C_{n}$.

Proposição 1.16. Sejam $C_{n}$ um grupo cíclico de ordem $n$ e $C_{n}=\langle g\rangle$. Então $u_{b c}$ definida acima é uma unidade de $\mathbb{Z} C_{n}$.

\section{Prova:}

Seja $x$ o menor inteiro positivo tal que $(b c) x \equiv 1(\bmod n)$, que existe pois $\operatorname{mdc}(b c, n)=1$. Afirmamos que

$$
v=\left(1+g+g^{2}+\cdots+g^{b-1}\right)\left(1+g+g^{2}+\cdots+g^{c-1}\right)\left(1+g^{b c}+g^{2 b c}+\cdots+g^{(x-1) b c}\right)+\frac{1-b c x}{n} \widehat{g}
$$

é o inverso de $u_{b c}$. De fato, observe que

$$
\left(1+g+g^{2}+\cdots+g^{b-1}\right) \cdot\left(1+g^{b}+g^{2 b}+\cdots+g^{b(a-1)}\right)=\left(1+g+g^{2}+\cdots+g^{a b-1}\right)=1+\frac{a b-1}{n} \widehat{g}
$$


e

$\left(1+g+g^{2}+\cdots+g^{c-1}\right)\left(1+g^{b c}+g^{2 b c}+\cdots+g^{(x-1) b c}\right) \cdot\left(1+g^{c}+g^{2 c}+\cdots+g^{(b-1) c}\right)=1+\frac{b c x-1}{n} \widehat{g}$

Assim

$v \cdot u_{b c}=\left(1+\frac{a b-1}{n} \widehat{g}\right) \cdot\left(1+\frac{b c x-1}{n} \widehat{g}\right)+b c x \frac{(1-a b)}{n} \widehat{g}+a b \frac{(1-b c x)}{n} \widehat{g}+\frac{(1-b c x)}{n}(1-a b) \widehat{g}=1$

Logo $v=\left(u_{b c}\right)^{-1}$.女子

Em livros, dependendo do autor, podemos também encontrar esta unidade descrita pela seguinte identidade:

$$
u_{b c}=\frac{1+g^{c}+g^{2 c}+\cdots+g^{c(b-1)}}{1+g+g^{2}+\cdots+g^{b-1}},
$$

onde $b, c \in \mathbb{Z}$ tais que $m d c(b c, n)=1$.

Proposição 1.17. Sejam $C_{n}$ um grupo cíclico de ordem $n$ e $C_{n}=\langle g\rangle$. Então vale a igualdade

$u_{b c}=\left(1+g^{b}+g^{2 b}+\cdots+g^{b(a-1)}\right)\left(1+g^{c}+g^{2 c}+\cdots+g^{c(b-1)}\right)+\frac{1-a b}{n} \widehat{g}=\frac{1+g^{c}+g^{2 c}+\cdots+g^{c(b-1)}}{1+g+g^{2}+\cdots+g^{b-1}}$

onde $a, b, c \in \mathbb{Z}$ são tais que $\operatorname{mdc}(b c, n)=1$ e $a b \equiv 1(\bmod n)$.

\section{Prova:}

Primeiramente, verificamos que se $m d c(n, b)=1$, então o elemento $1+g+g^{2}+\cdots+g^{b-1}$ tem inverso em $\mathbb{Q} C_{n}$. Seja $a \in \mathbb{Z}$ tal que $b a \equiv 1(\bmod n)$, digamos que $b a=1+k n$. Assim

$$
\begin{aligned}
& \left(1+g+g^{2}+\cdots+g^{b-1}\right) \cdot\left[1+g^{b}+g^{2 b}+\cdots+g^{b(a-1)}-\frac{k}{b} \widehat{g}\right]= \\
& =1+g+g^{2}+\cdots+g^{b-1}+g^{b}+\cdots+g^{b(a-1)}+\cdots+g^{b a-1}-\left(1+g+g^{2}+\cdots+g^{b-1}\right) \cdot \frac{k}{b} \widehat{g}= \\
& =1+g+g^{2}+\cdots+g^{b-1}+g^{b}+\cdots+g^{b(a-1)}+\cdots+g^{k n}-b \cdot \frac{k}{b} \widehat{g}=1+k \widehat{g}-k \widehat{g}=1 .
\end{aligned}
$$

$\operatorname{Logo}\left(1+g+g^{2}+\cdots+g^{b-1}\right)^{-1}=\left[1+g^{b}+g^{2 b}+\cdots+g^{b(a-1)}-\frac{k}{b} \widehat{g}\right]$. 
Agora

$$
\begin{aligned}
\frac{1+g^{c}+g^{2 c}+\cdots+g^{c(b-1)}}{1+g+g^{2}+\cdots+g^{b-1}} & =\left(1+g^{c}+\cdots+g^{c(b-1)}\right) \cdot\left(1+g+g^{2}+\cdots+g^{b-1}\right)^{-1} \\
& =\left(1+g^{c}+\cdots g^{c(b-1)}\right) \cdot\left[\left(1+g^{b}+\cdots+g^{b(a-1)}\right)-\frac{k}{b} \widehat{g}\right] \\
& =\left(1+g^{c}+\cdots g^{c(b-1)}\right) \cdot\left(1+g^{b}+\cdots+g^{b(a-1)}\right)-b \frac{k}{b} \widehat{g} \\
& =\left(1+g^{c}+\cdots g^{c(b-1)}\right) \cdot\left(1+g^{b}+\cdots+g^{b(a-1)}\right)-k \widehat{g} .
\end{aligned}
$$

Portanto, $\frac{1+g^{c}+g^{2 c}+\cdots+g^{c(b-1)}}{1+g+g^{2}+\cdots+g^{b-1}}=\left(1+g^{c}+\cdots g^{c(b-1)}\right) \cdot\left(1+g^{b}+\cdots+g^{b(a-1)}\right)-k \widehat{g}$. ¿ t

Vamos agora prosseguir com outros resultados conhecidos de unidades de anéis de grupo.

Definição 1.13. Seja $R G$ um anel de grupo. Considere o homomorfismo de anéis

$$
\begin{gathered}
R G \quad \stackrel{\epsilon}{\longrightarrow} \quad R \\
\left(\sum_{g \in G} a_{g} g\right) \stackrel{\longmapsto}{\longmapsto} \sum_{g \in G} a_{g}
\end{gathered}
$$

Tal homomorfismo é chamado de função de aumento de $R G$. Seu núcleo, representado por $\Delta(G)$, é chamado de ideal de aumento de $R G$.

Denotamos por $U_{1}(R G)$ o subgrupo das unidades de aumento 1 em $U(R G)$, isto é,

$$
U_{1}(R G):=\{u \in U(R G): \epsilon(u)=1\}
$$

O seguinte teorema estabelece uma relação entre $U(R G)$ e $U_{1}(R G)$.

Proposição 1.18. Seja $R G$ um anel de grupo. Então $U_{1}(R G)$ é um subgrupo normal de $U(R G)$ tal que

$$
U(R G)=U(R) \times U_{1}(R G)
$$

\section{Prova:}

O subgrupo $U_{1}(R G)$ é normal em $U(R G)$ pelo fato de $\epsilon: R G \longrightarrow R$ ser um homomorfismo. Evidentemente, $U(R) \cap U_{1}(R G)=1$. Observe também que, para qualquer $u \in U(R G), r=\epsilon(u)$ é uma unidade tal que $\epsilon\left(r^{-1} u\right)=1$. Logo a igualdade $u=r\left(r^{-1} u\right)$ implica em $U(R G)=U(R)$. $U_{1}(R G)$, concluindo a prova. is 
Em particular, como $U(\mathbb{Z})=\{-1,1\}$, então $U(\mathbb{Z} G)= \pm U_{1}(\mathbb{Z} G)$.

Para qualquer grupo abeliano $G$, existe uma fórmula para calcular o posto do subgrupo livre de torção de $U(\mathbb{Z} G)$. Este é um resultado bem conhecido que enunciaremos a seguir.

Teorema 1.19 (Teorema 4.5, [27] - Higman, Ayoub-Ayoub, May). Seja G um grupo abeliano arbitrário e seja $G_{0}$ o seu subgrupo de torção. Então

$$
U(\mathbb{Z} G)= \pm G \times F
$$

onde F é um grupo abeliano livre cujo posto é dado do seguinte modo:

$$
\text { posto } F= \begin{cases}\frac{1}{2}\left(\left|G_{0}\right|-2 l+m+1\right) & \text { se }\left|G_{0}\right| \text { é finito } \\ 0 & \text { se } G_{0}^{4}=1 \text { ou } G_{0}^{6}=1 \\ \left|G_{0}\right| & \text { se } G_{0} \text { é infinito, } G_{0}^{4} \neq 1 \text { e } G_{0}^{6} \neq 1\end{cases}
$$

onde $\left.G_{0}^{i}=\left\{g^{i} \mid g \in G_{0}\right)\right\}, m$ (respectivamente, l) é o número de subgrupos cíclicos de $G_{0}$ de ordem 2 (respectivamente, o número de subgrupos cíclicos de $G_{0}$ ).

Exemplo 1.6. Seja $G=C_{p^{n}}$ um grupo cíclico de ordem $p^{n}$, onde $p$ é um número primo ímpar $e$ $0 \neq n \in \mathbb{N}$. Neste caso $m=0$ e $l=n+1$ e, portanto,

$$
\text { posto } U(\mathbb{Z} G)=\frac{1}{2}\left(p^{n}-2(n+1)+1\right) \text {. }
$$

Exemplo 1.7. Se $G=\mathbb{Z}_{p} \times \mathbb{Z}_{p}$, para um primo $p \geq 5$, então $m=0$ e $l=p+2$. Então,

$$
\text { posto } U(\mathbb{Z} G)=\frac{1}{2}(p+1)(p-3) \text {. }
$$

Definição 1.14. Seja $R G$ um anel de grupo. Considere a função $*: R G \rightarrow R G$ definida por $\left(\sum_{g \in G} a_{g} g\right)^{*}=\sum_{g \in G} a_{g} g^{-1}$. Tal função é um anti-automorfismo chamada involução clássica.

Definição 1.15. O conjunto $U_{*}(R G):=\left\{u \in U_{1}(R G): u^{*}=u\right\}$ é chamado conjunto das unidades simétricas normalizadas de $R G$.

Este conjunto nem sempre tem a estrutura de um grupo. No entanto, a proposição abaixo estabelece uma condição que o torna um grupo. 
Proposição 1.20. Sejam $R$ um anel comutativo e $G$ um grupo abeliano. Então $U_{*}(R G)$ é um subgrupo de $U_{1}(R G)$.

\section{Prova:}

Sejam $u_{1}, u_{2} \in U_{*}(R G)$. Então $u_{i}^{*}=u_{i}$, para $i=1,2$. É claro que $\epsilon\left(u_{1} u_{2}\right)=1$. Por $R$ e $G$ serem comutativos, segue que $\left(u_{1} u_{2}\right)^{*}=u_{2}^{*} u_{1}^{*}=u_{2} u_{1}=u_{1} u_{2}$, ou seja, $u_{1}^{*} u_{2}^{*} \in U_{*}(R G)$. Ainda, se $u \in U_{*}(R G)$, então $\left(u^{-1}\right)^{*} u^{*}=\left(u u^{-1}\right)^{*}=1 \mathrm{e}$, portanto, $\left(u^{*}\right)^{-1}=\left(u^{*}\right)^{-1}$. Logo $U_{*}(R G)$ é um subgrupo de $U_{1}(R G)$. \&

Neste trabalho utilizamos as unidades simétricas, pois elas facilitam alguns cálculos. Pudemos observar que os resultados utilizando estas unidades reduziam o tempo de cálculo, comparada a uma unidade não simétrica, em programas como o GAP. Vejamos, então, porque podemos somente trabalhar com as unidades simétricas.

Proposição 1.21. Seja $u \in U(\mathbb{Z} G)$ tal que $u^{*} u=1$. Então $u= \pm g, g \in G$.

\section{Prova:}

Seja $u=\sum_{g \in G} u_{g} g$. Então $u^{*}=\sum_{g \in G} u_{g} g^{-1}$ e temos $1=u^{*} u=\left(\sum_{g \in G} u_{g}^{2}\right) \cdot 1+\sum_{g \neq 1} c_{g} g$, para algum $c_{g} \in \mathbb{Z}$. Logo $\sum_{g \in G} u_{g}^{2}=1$ e deste fato segue que $u_{g_{0}}= \pm 1$, para um único $g_{0} \in G$ e $u_{h}=0$, para todo $h \neq g_{0}$ em $G$. Portanto, provamos que $u= \pm g_{0}$. is

Teorema 1.22. Seja $G$ um grupo abeliano finito de ordem ímpar. Então

$$
U_{1}(\mathbb{Z} G)=G \times U_{*}(R G)
$$

\section{Prova:}

Seja $u \in U_{1}(\mathbb{Z} G)$. Observe que $\left(u^{-1} u^{*}\right)^{*}=u\left(u^{-1}\right)^{*}=\left(u^{-1}\right)^{*} u=\left(u^{-1} u^{*}\right)^{-1}$ e, portanto, $\left(u^{-1} u^{*}\right)^{*}\left(u^{-1} u^{*}\right)=1$. Como $u^{-1} u^{*} \in U_{1}(\mathbb{Z} G)$, pela Proposição $1.21, u^{-1} u^{*}=g^{-1}$, para algum $g \in G$, isto é, $u=g u^{*}$. Como $G$ tem ordem ímpar, podemos escrever $g=\left(g^{\frac{o(g)+1}{2}}\right)^{2}$, onde $o(g)$ denota a ordem de $g$. Seja $h=g^{\frac{o(g)+1}{2}}$ e, portanto, $u=h^{2} u^{*}$, ou seja, $h^{-1} u=h u^{*}$. Vejamos que $h u^{*} \in U_{*}(\mathbb{Z} G)$. Temos $\left(h u^{*}\right)^{*}=u h^{*}=u h^{-1}=h^{-1} u=h u^{*}$ e, portanto, $h u^{*} \in U_{*}(\mathbb{Z} G)$. Assim $u=h\left(h u^{*}\right)$, onde $h \in G$ e $h u^{*} \in U_{*}(\mathbb{Z} G)$.

Vejamos agora que $G \cap U_{*}(\mathbb{Z} G)=\{1\}$. Seja $g \in G \cap U_{*}(\mathbb{Z} G)$. Temos $g=g^{*}=g^{-1}$ e daí $g^{2}=1$. Como a ordem de $G$ é ímpar, temos $g=1$. Logo $G \cap U_{*}(\mathbb{Z} G)=(1)$ e, portanto, $U_{1}(\mathbb{Z} G)=G \times U_{*}(R G)$. ¿ $\times$ 
Note que pelos Teoremas 1.18 e 1.22, para encontrar um conjunto de geradores independentes das unidades de $U(\mathbb{Z} G)$, com $G$ abeliano finito de ordem ímpar, basta utilizar as unidades simétricas. No caso de um grupo abeliano finito arbitrário vale o teorema abaixo.

Proposição 1.23 (Proposição 8.3.4, [39]). Seja $G$ um grupo abeliano finito e $g \in G$. Se $(g-1) \in$ $(\Delta G)^{2}$, então $g=1$.

Teorema 1.24. Seja G um grupo abeliano finito. Então

$$
U_{1}(\mathbb{Z} G)=G \times U_{2}(\mathbb{Z} G)
$$

onde $U_{2}(\mathbb{Z} G)=\left\{u \in U(\mathbb{Z} G): u \equiv 1 \bmod (\Delta G)^{2}\right\}$. Mais ainda,

$$
U_{2}(\mathbb{Z} G) \subseteq U_{*}(G)=\left\{u \in U(\mathbb{Z} G): u^{*}=u\right\}
$$

\section{Prova:}

Seja $u \in U_{1}(\mathbb{Z} G)$. Então $u=1+\sum z_{g}(g-1)$, para $g \in G, z_{g} \in \mathbb{Z}$. Para quaisquer $x, y \in G$ podemos usar a identidade

$$
(x y-1)=(x-1)+(y-1)+(x-1)(y-1) \equiv(x-1)+(y-1) \bmod (\Delta G)^{2} .
$$

Indutivamente, mostramos a identidade de um modo mais geral. Se $x_{1}, x_{2}, \cdots, x_{n} \in G$, então

$$
\left(x_{1} \cdot x_{2} \cdots x_{n}-1\right) \equiv\left(x_{1}-1\right)+\left(x_{2}-1\right)+\cdots+\left(x_{n}-1\right) \bmod (\Delta G)^{2} \text {. }
$$

Em particular,

$$
\left(x^{n}-1\right) \equiv n(x-1) \bmod (\Delta G)^{2} .
$$

Como $u=1+\sum z_{g}(g-1)$, então temos $u \equiv 1+\sum\left(g^{z_{g}}-1\right)$ pelo que acabamos de afirmar. Aplicando novamente a identidade acima, temos $\sum\left(g^{z_{g}}-1\right) \equiv\left(\prod g^{z_{g}}\right)-1 \bmod (\Delta G)^{2}$, $\operatorname{logo} u \equiv 1+\left(\prod g^{z_{g}}\right)-1 \equiv \prod g^{z_{g}} \bmod (\Delta G)^{2}$. Seja $\prod g^{z_{g}}=g_{0} \in G$, então podemos escrever $u \equiv g_{0} \bmod (\Delta G)^{2}$.

Assim $u g_{0}^{-1} \equiv 1 \bmod (\Delta G)^{2}$ e, portanto $u g_{0}^{-1} \in U_{2}(\mathbb{Z} G)$. Logo $U_{1}(\mathbb{Z} G)=G \cdot U_{2}(\mathbb{Z} G)$. Resta provar que este é um produto direto. Suponha que temos um elemento $g \in G \operatorname{com} g \in U_{2}(\mathbb{Z} G)$. Então $g-1 \in(\Delta G)^{2}$ e $g=1$, pela Proposição 1.23. Isto completa a primeira parte do teorema. 
Para a segunda parte, seja $u \in U_{2}(\mathbb{Z} G)$. Considere $v=u^{-1} u^{*}$. Então $v^{-1}=\left(u^{-1} u^{*}\right)^{-1}=$ $u\left(u^{*}\right)^{-1}=v^{*}$. Pela Proposição 1.21, $v \in G$. Como $u \in U_{2}(\mathbb{Z} G)$ temos $(v-1) \in(\Delta G)^{2}$ e $v=1$ e, portanto, $u^{*}=u$ como queríamos. is

Proposição 1.25 (Lema 8.6.4, [28]). Sejam $C_{p^{n}}=\langle g\rangle$ um grupo cíclico de ordem $p^{n}$, $p$ um primo impar e $\theta$ uma raiz $p^{n}$-ésima primitiva da unidade. Então a aplicação

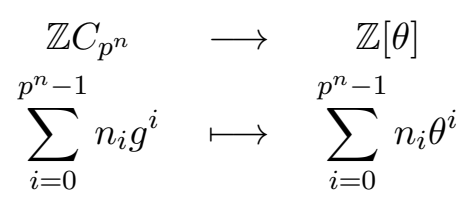

é um homomorfismo sobrejetor, cujo núcleo é um ideal principal gerado por $\sum_{i=0}^{p-1} g^{p^{n-1} i}$.

Proposição 1.26 (Lema 8.6.5, [28]). Sejam $G=\langle g\rangle$ um grupo cíclico de ordem $p^{n-1}, n \geq 1$, p primo impar e $\theta$ uma raiz $p^{n}$-ésima primitiva da unidade. Então a aplicação $\theta \mapsto g$ induz um homomorfismo sobrejetor $\pi: \mathbb{Z}[\theta] \rightarrow \mathbb{F}_{p} G$, onde $\mathbb{F}_{p}$ é um corpo com $p$ elementos. Além disso, o núcleo de $\pi$ é um ideal principal gerado por $\theta^{p^{n-1}}-\theta^{-p^{n-1}}$.

Proposição 1.27 (Lema 8.6.6, [28]). Sejam $A=\langle a\rangle$ e $B=\langle b\rangle$ grupos cíclicos de ordem $p^{n} e$ $p^{n-1}$, respectivamente, onde $n \geq 1, p$ um primo ímpar e $\theta$ uma raiz $p^{n}$-ésima primitiva da unidade. Então a aplicação

$$
\begin{array}{ccc}
\mathbb{Z} A & \longrightarrow & \mathbb{Z}[\theta] \times \mathbb{Z} B \\
\sum_{i=0}^{p^{n}-1} n_{i} a^{i} & \longmapsto & \left(\sum_{i=0}^{p^{n}-1} n_{i} \theta^{i}, \sum_{i=0}^{p^{n}-1} n_{i} b^{i}\right)
\end{array}
$$

induz um isomorfismo de $\mathbb{Z} A$ com o subanel $S$ de $\mathbb{Z}[\theta] \times \mathbb{Z} B$ dado por

$$
S=\{(x, y) \mid \pi(x)=\rho(y)\}
$$

onde o homomorfismo $\pi: \mathbb{Z}[\theta] \rightarrow \mathbb{F}_{p} B$ é definido por $\pi(\theta)=b$ e $\rho: \mathbb{Z} B \rightarrow \mathbb{F}_{p} B$ é o homomorfismo natural.

Proposição 1.28. Sejam $A=\langle a\rangle$ e $B=\langle b\rangle$ grupos cíclicos de ordem $p^{n}$ e $p^{n-1}$, respectivamente, onde $n \geq 1$, p um primo ímpar e $\theta$ uma raiz $p^{n}$-ésima primitiva da unidade. Então

$$
U(\mathbb{Z} A)=\left\{\sum_{i=0}^{p^{n}-1} n_{i} a^{i} \in \mathbb{Z} A: \sum_{i=0}^{p^{n}-1} n_{i} \theta^{i} \in U(\mathbb{Z}[\theta]), \sum_{i=0}^{p^{n}-1} n_{i} b^{i} \in U(\mathbb{Z} B)\right\} .
$$




\section{Prova:}

Sejam $\pi_{1}: \mathbb{Z} A \longrightarrow \mathbb{Z}[\theta]$ e $\pi_{2}: \mathbb{Z} A \longrightarrow \mathbb{Z} B$ homomorfismos de anéis definidos por

$$
\pi_{1}\left(\sum_{i=0}^{p^{n}-1} n_{i} a^{i}\right)=\sum_{i=0}^{p^{n}-1} n_{i} \theta^{i} \text { e } \pi_{2}\left(\sum_{i=0}^{p^{n}-1} n_{i} a^{i}\right)=\sum_{i=0}^{p^{n}-1} n_{i} b^{i}
$$

Então mostraremos que $U(\mathbb{Z} A)=\left\{u \in \mathbb{Z} A: \pi_{1}(u) \in U(\mathbb{Z}[\theta])\right.$ e $\left.\pi_{2}(u) \in U(\mathbb{Z} B)\right\}$.

É claro que $U(\mathbb{Z} A) \subseteq\left\{u \in \mathbb{Z} A: \pi_{1}(u) \in U(\mathbb{Z}[\theta])\right.$ e $\left.\pi_{2}(u) \in U(\mathbb{Z} B)\right\}$ e, portanto, verificaremos a outra inclusão.

Seja $u=\sum_{i=0}^{p^{n}-1} n_{i} a^{i} \in \mathbb{Z} A$ tal que $\pi_{1}(u) \in U(\mathbb{Z}[\theta])$ e $\pi_{2}(u) \in U(\mathbb{Z} B)$. Logo $\pi_{(}\left(\pi_{1}(u)\right)=\rho\left(\pi_{2}(u)\right)$ e daí $\pi\left(\left(\pi_{1}(u)\right)^{-1}\right)=\rho\left(\left(\pi_{2}(u)\right)^{-1}\right)$. Assim $\left(\left(\pi_{1}(u)\right)^{-1},\left(\pi_{2}(u)\right)^{-1}\right) \in S$, ou seja, existe $w \in \mathbb{Z} A$ tal que $\pi_{1}(w)=\left(\pi_{1}(u)\right)^{-1}$ e $\pi_{2}(w)=\left(\pi_{2}(u)\right)^{-1}$. Mostraremos que $w \cdot u=1$.

Observe que

$\pi_{1}(w \cdot u)=\pi_{1}(w) \cdot \pi_{1}(u)=\left(\pi_{1}(u)\right)^{-1} \cdot \pi_{1}(u)=1$ e $\pi_{2}(w \cdot u)=\pi_{2}(w) \cdot \pi_{2}(u)=\left(\pi_{2}(u)\right)^{-1} \cdot \pi_{2}(u)=1$.

Então $\left(\pi_{1}(w \cdot u), \pi_{2}(w \cdot u)\right)=(1,1) \in S$. Como existe uma bijeção entre $S$ e $\mathbb{Z} A$, resulta $w \cdot u=1$. Assim, $u \in U(\mathbb{Z} A)$. ¿s 


\section{Unidades de anéis de grupo modular}

Neste capítulo estudaremos brevemente o grupo das unidades de um anel de grupo modular. Um anel de grupo modular é um anel de um grupo sobre um anel de característica prima. Um dos mais difíceis e importantes problemas sobre álgebras de grupo modular consiste em descrever a estrutura do conjunto das unidades normalizadas e do conjunto dos automorfismos do grupo das unidades normalizadas.

Na álgebra de grupo modular de um p-grupo abeliano finito, o conjunto das unidades normalizadas é um p-grupo finito e uma base é conhecida no caso cíclico. Veremos mais a diante esta caracterização.

A motivação para estudarmos tal anel de grupo se deve ao fato de utilizarmos o seguinte diagrama comutativo:



onde $f_{1}$ é o homomorfismo natural e $f_{2}(\theta)=h$, com $C_{p^{n-1}}=\langle h\rangle$.

As ferramentas deste capítulo serão utilizadas no Capítulo 4, onde o diagrama mencionado será de grande importância.

\subsection{Unidades de $\mathbb{Z}_{p} C_{p^{n}}$}

Resultados a respeito de uma base para $U_{1}(K G)$ apareceram na literatura da $K$-Teoria Algébrica, como por exemplo em [3]. Janusz também encontrou a mesma base citada em [3] para 
$U_{1}(K G)$ no artigo [21]. Para esta tese, o que realmente nos interessa neste capítulo não é encontrar uma base para $U_{1}\left(\mathbb{Z} C_{p^{n}}\right)$, mas ao final do capítulo exibiremos uma tal base pois é um assunto interessante. Estamos sim, à procura de subconjuntos de $U_{1}\left(\mathbb{Z}_{p} C_{p^{n}}\right)$ que são multiplicativamente independentes, para determinarmos uma base de $\operatorname{Im}\left(\left.f_{1}\right|_{U_{1}\left(\mathbb{Z} C_{p^{n-1}}\right)}\right)$. Usaremos as Proposições 2.2 e 2.3 para determinar tais elementos.

Sejam $C_{p^{n}}=\langle g\rangle, u=c_{0}+c_{1} g+c_{2} g^{2}+\cdots+c_{p^{n}-1} g^{p^{n}-1} \in \mathbb{Z}_{p} C_{p^{n}}$ e $x=g-1$.

Sabemos que $x$ é um elemento nilpotente em $\mathbb{Z}_{p} C_{p^{n}}$ com índice de nilpotência $p^{n}$. Substituimos $g$ por $x+1$ em $u$, obtendo

$$
u=c_{0}+c_{1}(x+1)+c_{2}(x+1)^{2}+\cdots+c_{p^{n}-1}(x+1)^{p^{n}-1}
$$

e trabalharemos somente em função do nilpotente $x$. Como os coeficientes $c_{0}, c_{1}, \cdots, c_{p^{n}-1}$ acima são únicos, então existem únicos $a_{1}, a_{1}, \cdots, a_{p^{n}-1} \in \mathbb{Z}$ tais que

$$
u=a_{0}+a_{1} x+a_{2} x^{2}+\cdots+a_{p^{n}-1} x^{p^{n}-1}, a_{i} \in \mathbb{Z}
$$

Quando trabalhamos com as potências de um elemento de $\mathbb{Z}_{p} C_{p^{n}}$, há uma certa vantagem em utilizar $x$ em vez de $g$, pois não precisamos nos importar com os coeficientes dos $x^{i}$, quando $i \geq p^{n}$.

Proposição 2.1. Denote por $u_{i}=1+a_{i, 0} x^{i}+a_{i, 1} x^{i+1}+a_{i, 2} x^{i+2}+\cdots+a_{i, t} x^{p^{n}-1} \in \mathbb{Z}_{p} C_{p^{n}}$, com $1 \leq i \leq p^{n}-1, t=p^{n}-1-i, a_{i, j} \in \mathbb{Z}_{p}, a_{i, 0} \neq 0$ e $x=g-1$. Então valem os seguintes itens:

(i) Seja $c \in \mathbb{N}$ tal que p não divide $c$, então $u_{i}^{c}=1+c a_{i, 0} x^{i}+\beta_{1} x^{i+1}+\beta_{2} x^{i+2}+\cdots+\beta_{p^{n}-i-1} x^{p^{n}-1}$, onde $\beta_{l} \in \mathbb{Z}_{p}$.

(ii) Sejam $i, j \in \mathbb{N}$ com $i<j$, então $u_{i} \cdot u_{j}=1+a_{i, 0} x^{i}+\beta_{1} x^{i+1}+\beta_{2} x^{i+2}+\cdots+\beta_{p^{n}-i-1} x^{p^{n}-1}$, onde $\beta_{l} \in \mathbb{Z}_{p}$.

(iii) Se $p^{r} \leq i<p^{r+1}$ com $r \geq 0$, então a ordem de $u_{i}$ é $p^{n-r}$.

\section{Prova:}

Provaremos somente a propriedade (iii), pois (i) e (ii) são de fácil demonstração. O que queremos ressaltar em (i) é que a menor potência de $x$, diferente de 0 , em $u_{i}^{c}$ é ainda $x^{i}$ e o coeficiente associado a $x^{i}$ é $c \cdot a_{i, 0}$. E na propriedade (ii), a menor potência de $x$ no produto $u_{i} \cdot u_{j}$ é $m=\min \{i, j\}$. 
(iii) Primeiramente provaremos $\left(u_{i}\right)^{p^{n-r}}=1$.

$$
\begin{aligned}
\left(u_{i}\right)^{p^{n-r}} & =\left(1+a_{i, 0} x^{i}+a_{i, 1} x^{i+1}+a_{i, 2} x^{i+2}+\cdots+a_{i, t} x^{i+t}\right)^{p^{n-r}} \\
& =1+a_{i, 0}^{p^{n-r}} x^{i p^{n-r}}+a_{i, 1}^{p^{n-r}} x^{(i+1) p^{n-r}}+a_{i, 2}^{p^{n-r}} x^{(i+2) p^{n-r}}+\cdots+a_{i, t}^{p^{n-r}} x^{(i+t) p^{n-r}} \\
& =1,
\end{aligned}
$$

pois $x^{i p^{n-r}}=0$, já que $p^{r} \leq i<p^{r+1}$.

Como $\left(u_{i}\right)^{p^{n-r}}=1$ para $p^{r} \leq i<p^{r+1}$, então a ordem de $u_{i}$ divide $p^{n-r}$. Se mostrarmos que $\left(u_{i}\right)^{p^{n-r-1}} \neq 1$, teremos provado que a ordem de $u_{i}$ é $p^{n-r}$.

$$
\begin{aligned}
\left(u_{i}\right)^{p^{n-r-1}} & =\left(1+a_{i, 0} x^{i}+a_{i, 1} x^{i+1}+a_{i, 2} x^{i+2}+\cdots+a_{i, t} x^{i+t}\right)^{p^{n-r-1}} \\
& =1+a_{i, 0}^{p^{n-r-1}} x^{i p^{n-r-1}}+a_{i, 1}^{p^{n-r-1}} x^{(i+1) p^{n-r-1}}+\cdots+a_{i, t}^{p^{n-r-1}} x^{(i+t) p^{n-r-1}} \\
& \neq 1,
\end{aligned}
$$

pois $a_{i, 0}^{p^{n-r-1}}=a_{i, 0} \neq 0$, já que $a_{i, 0} \neq 0$, e $x^{i p^{n-r-1}} \neq 0$, pois por hipótese $p^{r} \leq i<p^{r+1}$. Concluímos deste modo a prova de(iii). is

Se $A$ é um grupo (abeliano) finito, todo elemento de $A$ é de torção, logo não poderíamos falar em subconjunto multiplicativamente independente da forma usual. No entanto, se $A$ for um $p$-grupo definiremos um subconjunto multiplicativamente independente do seguinte modo:

Definição 2.1. Um conjunto $\left\{u_{1}, u_{2}, \cdots, u_{k}\right\}$ é multiplicativamente independente num p-grupo abeliano finito se

$$
\left\langle u_{i}\right\rangle \cap\left\langle u_{1}, u_{2}, \cdots, u_{i-1}, u_{i+1}, \cdots, u_{k}\right\rangle=\{1\}, \quad \text { para todo } 1 \leq i \leq k .
$$

Ou, se $u_{1}^{r_{1}} u_{1}^{r_{2}} \cdots u_{k}^{r_{k}}=1$ implicar $u_{j}^{r_{j}}=1$, para todo $1 \leq j \leq k$.

Equivalentemente podemos dizer que $\left\{u_{1}, u_{2}, \cdots, u_{k}\right\}$ é multiplicativamente independente se, e somente se, $\left\langle u_{1}\right\rangle \cdot\left\langle u_{2}\right\rangle \cdots\left\langle u_{k}\right\rangle=\left\langle u_{1}\right\rangle \times\left\langle u_{2}\right\rangle \times \cdots \times\left\langle u_{k}\right\rangle$.

Veremos a seguir elementos que são multiplicativamente independentes em $\mathbb{Z}_{p} C_{p^{n}}$.

Proposição 2.2. Sejam $C_{p^{n}}=\langle g\rangle, x=g-1$ e $u, v \in U_{1}\left(\mathbb{Z}_{p} C_{p^{n}}\right)$ da seguinte forma:

$$
u=1+a_{0} x^{p^{i} q}+a_{1} x^{p^{i} q+1}+a_{2} x^{p^{i} q+2}+\cdots+a_{t} x^{p^{n}-1}, \quad t=p^{n}-1-p^{i} q,
$$




$$
v=1+b_{0} x^{p^{j} r}+b_{1} x^{p^{j} r+1}+b_{2} x^{p^{j} r+2}+\cdots+b_{s} x^{p^{n}-1}, \quad s=p^{n}-1-p^{j} r
$$

onde $a_{0} \not \equiv 0(\bmod p), b_{0} \not \equiv 0(\bmod p)$ e, q e $r$ são inteiros distintos tais que $p \nmid q$ e $p \nmid r$. Então $\langle u\rangle \cap\langle v\rangle=\{1\}$.

\section{Prova:}

Vamos supor $u \neq 1$ e $v \neq 1$, pois senão não há nada a provar. Seja $w \in\langle u\rangle \cap\langle v\rangle$, então existem inteiros $n_{1}, n_{2}$ tais que:

$$
w=u^{n_{1}}=\left(1+a_{0} x^{p^{i} q}+a_{1} x^{p^{i} q+1}+a_{2} x^{p^{i} q+2}+\cdots+a_{t} x^{p^{n}-1}\right)^{n_{1}}
$$

e

$$
w=v^{n_{2}}=\left(1+b_{0} x^{p^{j} r}+b_{1} x^{p^{j} r+1}+b_{2} x^{p^{j} r+2}+\cdots+b_{s} x^{p^{n}-1}\right)^{n_{2}} .
$$

Sejam $n_{1}=p^{k} m_{1}$ e $n_{2}=p^{l} m_{2}$, com $m_{1}$ e $m_{2}$ inteiros tais que $p$ não não divide $m_{1}$ nem $m_{2}$. Logo

$$
\begin{aligned}
w=u^{n_{1}} & =\left(1+a_{0} x^{p^{i} q}+a_{1} x^{p^{i} q+1}+a_{2} x^{p^{i} q+2}+\cdots+a_{t} x^{p^{n}-1}\right)^{n_{1}} \\
& =\left(1+a_{0}^{p^{k}} x^{q p^{i+k}}+a_{1}^{p^{k}} x^{\left(q p^{i}+1\right) p^{k}}+a_{2}^{p^{k}} x^{\left(q p^{i}+2\right) p^{k}}+\cdots+a_{t}^{p^{k}} x^{\left(p^{n}-1\right) p^{k}}\right)^{m_{1}} \\
& =1+m_{1} x^{q p^{i+k}}+c_{1} x^{q p^{i+k}+1}+c_{2} x^{q p^{i+k}+2}+\cdots+c_{t^{\prime}} x^{p^{n}-1} .
\end{aligned}
$$

Por outro lado, temos

$$
\begin{aligned}
w=v^{n_{2}} & =\left(1+b_{0} x^{p^{j} r}+b_{1} x^{p^{j} r+1}+b_{2} x^{p^{j} r+2}+\cdots+b_{s} x^{p^{n}-1}\right)^{n_{2}} \\
& =\left(1+b_{0}^{p^{l}} x^{r p^{j+l}}+b_{1}^{p^{l}} x^{\left(p^{j} r+1\right) p^{l}}+b_{2}^{p^{l}} x^{\left(p^{j} r+2\right) p^{l}}+\cdots+b_{s}^{p^{l}} x^{\left(p^{n}-1\right) p^{l}}\right)^{m_{2}} \\
& =1+m_{2} x^{r p^{j+l}}+\beta_{1} x^{r p^{j+l}+1}+\beta_{2} x^{r p^{j+l}+2}+\cdots+\beta_{s^{\prime}} x^{p^{n}-1} .
\end{aligned}
$$

As igualdades (2.1) e (2.2) são válidas por causa da propriedade (i). Como $q \neq r$ e $p \nmid q$ e $p \nmid r$, então, $q p^{i+k} \neq r p^{j+l}$. Vamos analisar os seguintes casos:

- $q p^{i+k}<p^{n}$ e $r p^{j+l}<p^{n}$

- $q p^{i+k} \geq n$ ou $r p^{j+l} \geq n$.

O primeiro caso resulta em $x^{q p^{i+k}} \neq x^{r p^{k+l}}$. Então das igualdades (2.1) e (2.2) temos $m_{1} x^{q p^{i+k}}=0$ ou $m_{2} x^{r p^{j+l}}=0$. Como $p$ não divide nem $m_{1}$ nem $m_{2}$, devemos ter $x^{q p^{i+k}}=0$ ou $x^{r p^{j+l}}=0$, o que contradiz a hipótese. Logo não podemos ter este caso. 
Assim $q p^{i+k} \geq n$ ou $r p^{j+l} \geq n$ e isto implica $x^{q p^{i+k}}=0$ ou $x^{r p^{j+l}}=0$. Então $w=1$ pelas equações $(2.1)$ e (2.2). Logo $\langle u\rangle \cap\langle v\rangle=\{1\}$. ¿̇

Exemplo 2.1. Sejam $u, v \in \mathbb{Z}_{3} C_{27}$ que tenham a forma

$$
u=\overline{1}+\overline{2} x^{4}+\overline{2} x^{5}+x^{6}+x^{7}+x^{8}
$$

$e$

$$
v=\overline{1}+x^{6}+\overline{2} x^{8}
$$

Pela proposição anterior u e $v$ são multiplicativamente independentes.

De modo semelhante à proposição anterior, provamos o seguinte resultado:

Proposição 2.3. Sejam $I=\left\{i \in \mathbb{N} \mid 0<i<p^{n}\right.$ e $\left.p \nmid i\right\}$ e $u_{i}$ o elemento que definimos na Proposição 2.1. Então $\left\{u_{i} \mid i \in I\right\}$ é um conjunto multiplicativamente independente.

\section{Prova:}

Sabemos que $|I|=\phi\left(p^{n}\right)$, onde $\phi$ é a função de Euler. Vamos denotar a cardinalidade de $I$ por $d$ e $I=\left\{i_{1}, i_{2}, \cdots, i_{d-1}, i_{d}\right\}$. Mostraremos que

$$
\left\langle u_{i_{j}}\right\rangle \cap\left\langle u_{i_{1}}, u_{i_{2}}, \cdots, u_{i_{j-1}}, u_{i_{j+1}}, \cdots, u_{i_{d}}\right\rangle=\{1\} .
$$

Suponha $w \in\left\langle u_{i_{j}}\right\rangle \cap\left\langle u_{i_{1}}, u_{i_{2}}, \cdots, u_{i_{j-1}}, u_{i_{j+1}}, \cdots, u_{i_{d}}\right\rangle$. Então existem inteiros $m_{1}, m_{2}, \cdots, m_{d}$ tais que $w=u_{i_{j}}^{m_{j}}=u_{i_{1}}^{m_{1}} \cdot u_{i_{2}}^{m_{2}} \cdots u_{i_{j-1}}^{m_{j-1}} \cdot u_{i_{j+1}}^{m_{j+1}} \cdots u_{i_{d}}^{m_{d}}$. Decomponha $m_{k}$ do seguinte modo: $m_{k}=p^{l_{k}} c_{k}$, onde $p$ não divide $c_{k}$.

Como $i_{1}, i_{2}, \cdots, i_{d}$ são todos distintos e $p$ não divide $i_{j}$, segue que $i_{1} p^{l_{1}}, i_{2} p^{l_{2}}, \cdots, i_{d} p^{l_{d}}$ também são todos distintos. Logo podemos supor, sem perda de generalidade, que $i_{1} p^{l_{1}}<i_{2} p^{l_{2}}<\cdots<i_{d} p^{l_{d}}$. Assim

$$
\begin{aligned}
u_{i_{j}}^{m_{j}} & =u_{i_{1}}^{m_{1}} \cdot u_{i_{2}}^{m_{2}} \cdots u_{i_{j-1}}^{m_{j-1}} \cdot u_{i_{j+1}}^{m_{j+1}} \cdots u_{i_{d}}^{m_{d}} \\
\left(u_{i_{j}}\right)^{p^{l_{j}} c_{j}} & =\left(u_{i_{1}}\right)^{c_{1} p^{l_{1}}}\left(u_{i_{2}}\right)^{c_{2} p^{l_{2}}} \cdots\left(u_{i_{d}}\right)^{c_{d} p^{l_{d}}} \\
1+c_{j}\left(a_{i_{j}, 0}\right)^{p^{l_{j}}} x^{i_{j} p^{l_{j}}}+\cdots+\beta_{s} x^{p^{n}-1} & =1+c_{1}\left(a_{i_{1}, 0}\right)^{p^{l_{1}}} x^{\left(i_{1} p^{l_{1}}\right)}+\alpha_{1} x^{\left(i_{1} p^{l_{1}}+1\right)}+\cdots+\alpha_{t} x^{p^{n}-1},
\end{aligned}
$$


onde $s=p^{n}-1-i_{j} p^{l_{j}}$ e $t=p^{n}-1-i_{1} p^{l_{1}}$. Esta última igualdade segue da propriedade (i) da Proposição 2.1. Como $i_{1}$ e $i_{j}$ são distintos, então pela unicidade como os elementos são escritos devemos ter:

I) $x^{i_{1} p^{l_{1}}}=0$ e daí $x^{i_{k} p_{k}^{l_{k}}}=0$, para todo $k \geq 1$, pois estamos supondo $i_{1} p^{l_{1}}<i_{2} p^{l_{2}}<\cdots<i_{d} p^{l_{d}}$. $\log 0 w=u_{i_{j}}^{m_{j}}=1 \in\left\langle u_{i_{j}}\right\rangle \cap\left\langle u_{i_{1}}, u_{i_{2}}, \cdots, u_{i_{j-1}}, u_{i_{j+1}}, \cdots, u_{i_{d}}\right\rangle$.

$\mathrm{Ou}$

II) $x^{i_{1} p^{l_{1}}} \neq 0$ e daí $c_{1} \equiv c_{1}\left(a_{i_{1}, 0}\right)^{p^{l_{1}}} \equiv 0(\bmod p)$ pois $i_{1} p^{l_{1}}<i_{2} p^{l_{2}}<\cdots<i_{d} p^{l_{d}}$ e $w$ é escrito de modo único. Mas isto é um absurdo pois $p \nmid c_{1}$, logo não podemos ter $x^{i_{1} p^{l_{1}}} \neq 0$.

$\operatorname{Assim}\left\langle u_{i_{j}}\right\rangle \cap\left\langle u_{i_{1}}, u_{i_{2}}, \cdots, u_{i_{j-1}}, u_{i_{j+1}}, \cdots, u_{i_{d}}\right\rangle=\{1\}$ e, portanto, $\left\{u_{i} \mid i \in I\right\}$ é multiplicativamente independente. is

O nosso próximo objetivo é descrever o conjunto de geradores independentes de $U_{1}\left(\mathbb{Z}_{p} C_{p^{n}}\right)$.

Proposição 2.4. Seja $G$ um p-grupo finito e $K$ um corpo primo com p elementos. Então

$$
\left|U_{1}(K G)\right|=p^{|G|-1}
$$

\section{Prova:}

Seja $\epsilon$ a função de aumento $\epsilon: K G \rightarrow K$ e $\operatorname{ker}(\epsilon)=\Delta(G)$ o ideal de aumento. Um resultado bem conhecido é

$$
\Delta G=\langle\{g-1 \mid g \in G ; g \neq 1\}\rangle
$$

Em $U_{1}(K G)$, qualquer elemento que possui aumento 1 é unidade, logo $\langle 1+\Delta G\rangle \subseteq U_{1}(K G)$. Se restringirmos o domínio de $\epsilon$ ao subgrupo $U_{1}(K G)$, teremos um homomorfismo de grupos e pelo teorema do homomorfismo resulta $\frac{U_{1}(K G)}{\langle 1+\Delta G\rangle} \simeq\{1\}$, portanto, $\left|U_{1}(K G)\right|=p^{|G|-1}$. is

Como estamos interessados no grupo cíclico de ordem $p^{n}$, temos $\left|U_{1}\left(\mathbb{Z}_{p} C_{p^{n}}\right)\right|=p^{p^{n}-1}$.

Uma prova alternativa para teorema abaixo pode ser encontrada no livro de H. Bass [3] na página 602 e a prova é atribuída a Milnor. Iremos demonstrar de uma outra forma, que foi provada independentemente, pois encontramos a prova de Milnor posteriormente.

Teorema 2.5. Sejam $I=\left\{i \in \mathbb{N} \mid 0<i<p^{n}\right.$ e $\left.p \nmid i\right\}, C_{p^{n}}-\langle g\rangle$ um grupo cíclico de ordem $p^{n} e$ $x=g-1$. Então $U_{1}\left(\mathbb{Z}_{p} C_{p^{n}}\right)$ é produto direto de grupos cíclicos $B_{i}=\left\langle 1+x^{i}\right\rangle$, para $i \in I$. 


\section{Prova:}

Primeiramente observe que o elemento $1+x^{i}$ é um caso particular de $u_{i}$ que definimos anteriormente. Basta tomar $a_{i, 0}=1$ e $a_{i, j}=0$ para $j \neq 0$ em $u_{i}=1+a_{i, 0} x^{i}+a_{i, 1} x^{i+1}+a_{i, 2} x^{i+2}+\cdots+a_{i, t} x^{i+t}$. Sabemos que a ordem de $U_{1}\left(\mathbb{Z}_{p} C_{p^{n}}\right)$ é $p^{p^{n}-1}$. Provamos na Proposição 2.3 que o conjunto $\left\{u_{i} \mid i \in I\right\}$ é multiplicativamente independente, logo o produto $\prod_{i \in I} B_{i}$ é direto. Temos $B_{i} \subseteq U_{1}\left(\mathbb{Z}_{p} C_{p^{n}}\right)$, pois $1+x^{i}$ tem aumento 1 . Se mostrarmos que a ordem de $\prod_{i \in I} B_{i}$ é $p^{p^{n}-1}$, teremos concluído a nossa prova.

O inteiro $i$ nesta prova sempre será um elemento de $I$. Pela propriedade (iii) da Proposição 2.1, se $p^{r}<i<p^{r+1}$, então a ordem de $u_{i}$ é $p^{n-r}$, ou seja, $\left|B_{i}\right|=p^{n-r}$, para $p^{r}<i<p^{r+1}$.

Existem $\phi(p)$ inteiros $i$ entre 1 e $p$, ou seja, existem $\phi(p)$ unidades $u_{i}$ 's cuja ordem é $p^{n}$. Como $\left\{u_{i} \mid i \in I\right\}$ é multiplicativamente independente, temos $\left|\prod_{1 \leq i<p} B_{i}\right|=\left(p^{n}\right)^{\phi(p)}=p^{n \phi(p)}$.

Exitem $\phi\left(p^{2}\right)-\phi(p)$ inteiros $i \in\left\{j \in I \mid p<j<p^{2}\right\}$, logo existem $\phi\left(p^{2}\right)-\phi(p)$ unidades $u_{i}$ 's cuja ordem é $p^{n-1}$. Sendo assim, temos $\left|\prod_{p<i<p^{2}} B_{i}\right|=\left(p^{n-1}\right)^{\phi\left(p^{2}\right)-\phi(p)}$.

De modo geral, existem $\phi\left(p^{r+1}\right)-\phi\left(p^{r}\right)$ inteiros $i \in\left\{j \in I \mid p^{r}<i<p^{r+1}\right\}$, portanto existem $\phi\left(p^{r+1}\right)-\phi\left(p^{r}\right)$ unidades $u_{i}$ 's cuja ordem é $p^{n-r}$. Dai $\left|\prod_{p^{r}<i<p^{r+1}} B_{i}\right|=\left(p^{n-r}\right)^{\phi\left(p^{r+1}\right)-\phi\left(p^{r}\right)}$ e então

$$
\begin{aligned}
\left|\prod_{i \in I} B_{i}\right| & =p^{n \phi(p)+(n-1)\left(\phi\left(p^{2}\right)-\phi(p)\right)+(n-2)\left(\phi\left(p^{3}\right)-\phi\left(p^{2}\right)\right)+\cdots+2\left(\phi\left(p^{n-1}\right)-\phi\left(p^{n-2}\right)\right)+\left(\phi\left(p^{n}\right)-\phi\left(p^{n-1}\right)\right)} \\
& =p^{\phi(p)+\phi\left(p^{2}\right)+\phi\left(p^{3}\right)+\cdots+\phi\left(p^{n}\right)}
\end{aligned}
$$

Provaremos que $\phi(p)+\phi\left(p^{2}\right)+\phi\left(p^{3}\right)+\cdots+\phi\left(p^{n}\right)=p^{n}-1$ e, então teremos concluído a prova deste teorema.

Sabemos que $\sum_{d \mid m} \phi(d)=m$ e, portanto, $\sum_{d \mid p^{n}} \phi(d)=p^{n}$. Como $\sum_{d \mid p^{n}} \phi(d)=\sum_{0 \leq i \leq n} \phi\left(p^{i}\right)=p^{n}$, temos $\phi(p)+\phi\left(p^{2}\right)+\phi\left(p^{3}\right)+\cdots+\phi\left(p^{n}\right)=p^{n}-1$.

Deste modo, $\left|\prod_{i \in I} B_{i}\right|=p^{\left(p^{n}-1\right)} \mathrm{e}$, portanto, $U_{1}\left(\mathbb{Z}_{p} C_{p^{n}}\right)=\prod_{i \in I} B_{i}$. ¿े 


\section{CAPÍtulo 3}

\section{Unidades de $\mathbb{Z}[\theta]$}

Neste capítulo iremos provar alguns resultados a respeito de unidades do anel dos inteiros $\mathbb{Z}[\theta]$, sendo $\theta$ uma raiz $p^{n}$-ésima primitiva da unidade, onde $1 \leq n \in \mathbb{Z}$ e $p$ um número primo tal que o conjunto $\left\{ \pm \theta, \mu_{t} \mid 1<t<p^{n} / 2, m d c\left(p^{n}, t\right)=1\right\}$ gera $U(\mathbb{Z}[\theta])$.

Como foi dito anteriormente, este trabalho é uma extensão de resultados do artigo [15] de Ferraz, onde é considerado o caso $\mathbb{Z} C_{p}$, onde $p$ é um número primo ímpar entre 5 e 70.

Aqui os resultados a respeito das unidades de $\mathbb{Z}[\theta]$ são quase os mesmos do artigo [15], mas com algumas alterações. Iremos dividir este capítulo em 3 seções. Na primeira parte verificaremos algumas propriedades das unidades ciclotômicas e definiremos um homomorfismo auxiliar. Na segunda seção, restringiremos os resultados ao caso em que $p$ é um primo ímpar satisfazendo a condição $\phi\left(p^{n}\right) \leq 66$ e, na terceira seção, ao caso par.

\subsection{Unidades Ciclotômicas}

Iniciamos esta seção com algumas propriedades das unidades ciclotômicas, que serão utilizadas ao longo deste capítulo. Os resultados a seguir seguem para $\theta$ sendo uma raiz $p^{n}$-ésima primitiva da unidade, $p$ um número primo e $n$ um número natural diferente de zero. A condição $\phi\left(p^{n}\right) \leq 66$ será considerada mais adiante, pois os resultados a seguir valem de modo mais geral.

Sejam $i$ um inteiro positivo tal que $m d c\left(p^{n}, i\right)=1$ e $\mu_{i}=\frac{\theta^{i}-1}{\theta-1}$ uma unidade ciclotômica. Como $\theta^{i}-1=(\theta-1)\left(1+\theta+\theta^{2}+\cdots+\theta^{i-1}\right)$, podemos expressar a unidade ciclotômica como $\mu_{i}=1+\theta+\theta^{2}+\cdots+\theta^{i-1}$. Se $j$ é o inverso de $i$ módulo $p^{n}$, então $\mu_{i}^{-1}=1+\theta^{i}+\theta^{2 i}+\cdots+\theta^{i(j-1)}$.

Com estas notações verifiquemos as seguintes propriedades: 
P1. $1+\theta^{p}+\theta^{2 p}+\cdots+\theta^{\left(p^{n-1}-1\right) p}=0$.

P2. $i \equiv 0\left(\bmod p^{n}\right) \Longrightarrow \mu_{i}=0$.

P3. $1 \leq i \leq p^{n}-1 \Longrightarrow \mu_{i}=-\theta^{i} \mu_{p^{n}-i}$.

P4. $i+j \equiv 0\left(\bmod p^{n}\right) \Longrightarrow \mu_{i}=-\theta^{i} \mu_{j}$.

P5. $i \equiv j\left(\bmod p^{n}\right) \Longrightarrow \mu_{i}=\mu_{j}$.

\section{Prova:}

P1) Temos $\left(1-\theta^{p}\right)\left(1+\theta^{p}+\theta^{2 p}+\cdots+\theta^{\left(p^{n-1}-1\right) p}\right)=0$ e $\left(1-\theta^{p}\right) \neq 0$, pois $\theta$ é raiz $p^{n}$-ésima primitiva da unidade. Logo $1+\theta^{p}+\theta^{2 p}+\cdots+\theta^{\left(p^{n-1}-1\right) p}=0$.

P2) Como $\theta$ é raiz $p^{n}$-ésima primitiva da unidade, temos $1+\theta+\theta^{2}+\cdots+\theta^{p^{n}-1}=0$. Por hipótese, temos $i \equiv 0\left(\bmod p^{n}\right)$, então existe um $q \in \mathbb{Z}$ tal que $i=q p^{n}$. Deste modo

$$
\mu_{i}=1+\theta+\theta^{2}+\cdots+\theta^{p^{n}-1}+\theta^{p^{n}}+\theta^{p^{n}+1}+\cdots+\theta^{2 p^{n}-1}+\theta^{2 p^{n}}+\theta^{2 p^{n}+1}+\cdots+\theta^{q p^{n}-1},
$$

ou seja,

$$
\mu_{i}=\underbrace{\left(1+\theta+\theta^{2}+\cdots+\theta^{p^{n}-1}\right)+\left(1+\theta+\theta^{2}+\cdots+\theta^{p^{n}-1}\right)+\cdots+\left(1+\theta+\theta^{2}+\cdots+\theta^{p^{n}-1}\right)}_{\text {q vezes }}=0 .
$$

P3) Por (P2), temos $\mu_{p^{n}}=0$. Daí $\mu_{p^{n}}=1+\theta+\theta^{2}+\cdots+\theta^{i-1}+\theta^{i}+\theta^{i+1} \cdots+\theta^{p^{n}-1}=0$ e, portanto,

$\mu_{i}=1+\theta+\theta^{2}+\cdots+\theta^{i-1}=-\left(\theta^{i}+\theta^{i+1}+\cdots+\theta^{p^{n}-1}\right)=-\theta^{i}\left(1+\theta+\cdots+\theta^{p^{n}-i-1}\right)=-\theta^{i} \mu_{p^{n}-i}$.

P4) Novamente pela propriedade (P2) temos $\mu_{i+j}=0$, ou seja,

$$
1+\theta+\theta^{2}+\cdots+\theta^{i-1}+\theta^{i}+\theta^{i+1} \cdots+\theta^{i+j-1}=0
$$

Logo

$\mu_{i}=1+\theta+\theta^{2}+\cdots+\theta^{i-1}=-\left(\theta^{i}+\theta^{i+1}+\cdots+\theta^{i+j-1}\right)=-\theta^{i}\left(1+\theta+\theta^{2}+\cdots+\theta^{j-1}\right)=-\theta^{i} \mu_{j}$.

P5) Vamos supor, sem perda de generalidade, $i<j$. Como $i \equiv j\left(\bmod p^{n}\right)$, existe um $q \in \mathbb{Z}$ tal que $j=q p^{n}+i$. Então $\theta^{j}=\theta^{q p^{n}+i}=\theta^{q p^{n}} \theta^{i}=\theta^{i}$.

Ainda temos $j-i \equiv 0\left(\bmod p^{n}\right)$, assim pela propriedade (P2) e pela hipótese segue,

$$
\mu_{j-i}=1+\theta+\theta^{2}+\cdots+\theta^{i-1}+\theta^{i}+\theta^{i+1}+\cdots+\theta^{j-i-1}=0,
$$


isto é,

$$
\mu_{i}=1+\theta+\theta^{2}+\cdots+\theta^{i-1}=-\left(\theta^{i}+\theta^{i+1}+\cdots+\theta^{j-i-1}\right)=-\left(\theta^{j}+\theta^{j+1}+\cdots+\theta^{p^{n}-1}\right) .
$$

Lembrando que $\mu_{j}=1+\theta+\theta^{2}+\cdots \cdots+\theta^{j-1}=-\left(\theta^{j}+\theta^{j+1} \cdots+\theta^{p^{n}-1}\right)$, concluímos que $\mu_{i}=\mu_{j}$. ¿

Agora vamos definir um homomorfismo $\psi$ de grupos no qual o núcleo será importante no que segue. Para definí-lo utilizamos homomorfismos de anéis. Vejamos:

Considere o homomorfismo de anéis $\epsilon_{1}: \mathbb{Z}[x] \rightarrow \mathbb{Z}$ definido pela avaliação em $1: \epsilon_{1}(f(x))=f(1)$. Seja $\varpi: \mathbb{Z} \rightarrow \mathbb{Z}_{p}$ o homomorfismo canônico $\varpi(z)=\bar{z}$, para cada $z \in \mathbb{Z}$. Então temos a composição de homomorfismos de anéis:

$$
\begin{aligned}
& \mathbb{Z}[x] \stackrel{\epsilon_{1}}{\longrightarrow} \mathbb{Z} \quad \stackrel{\varpi}{\longrightarrow} \mathbb{Z}_{p} \\
& p(x) \quad \longmapsto p(1) \quad \longmapsto \overline{p(1)}
\end{aligned}
$$

Denotemos esta composta por $\rho: \mathbb{Z}[x] \rightarrow \mathbb{Z}_{p}$. Observe agora que o ideal gerado pelo $p^{n}$-ésimo polinômio ciclotômico $\Phi_{p}\left(x^{p^{n-1}}\right)=1+x^{p^{n-1}}+x^{2 p^{n-1}}+\cdots+x^{(p-1) p^{n-1}}$ está contido no núcleo de $\rho$ pois $\rho\left(\Phi_{p}\left(x^{p^{n-1}}\right)\right)=\bar{p}=\overline{0}$. Assim podemos definir o seguinte homomorfismo de anéis:

$$
\begin{aligned}
\bar{\rho}: \mathbb{Z}[x] /\left\langle\Phi_{p}\left(x^{p^{n-1}}\right)\right\rangle & \longrightarrow \mathbb{Z}_{p} \\
\overline{f(x)} & \longmapsto \bar{\rho}(f(x))=\rho(f(x)) .
\end{aligned}
$$

Facilmente se verifica que este homomorfismo está bem definido. Se $\overline{f_{1}(x)}=\overline{f_{2}(x)}$, então $f_{1}(x)-f_{2}(x) \in\left\langle\Phi_{p}\left(x^{p^{n-1}}\right)\right\rangle \subseteq \operatorname{ker}(\rho)$. Deste modo, $\rho\left(f_{1}(x)\right)-\rho\left(f_{2}(x)\right)=\rho\left(f_{1}(x)-f_{2}(x)\right)=0$, ou seja, $\rho\left(f_{1}(x)\right)=\rho\left(f_{2}(x)\right)$. Logo $\bar{\rho}\left(\overline{f_{1}(x)}\right)=\bar{\rho}\left(\overline{f_{2}(x)}\right)$ e daí $\bar{\rho}$ está bem definida. Como $\rho$ é homomorfismo de anéis temos que $\bar{\rho}$ é também um homomorfismo de anéis.

Seja

$$
\begin{aligned}
\epsilon_{\theta}: & \mathbb{Z}[x] \\
f(x) & \longmapsto \mathbb{Z}[\theta] \\
& \longmapsto f(\theta)
\end{aligned}
$$

a avaliação em $\theta$. Vimos no Capítulo 1 que $\Phi_{p^{n}}(x)$ é um polinômio minimal de $\theta$. Temos ainda 
$\Phi_{p^{n}}(x)=\Phi_{p}\left(x^{p^{n-1}}\right) \in \operatorname{ker}\left(\epsilon_{\theta}\right), \operatorname{logo} \operatorname{ker}\left(\epsilon_{\theta}\right)=\left\langle\Phi_{p^{n}}(x)\right\rangle$ e, pelo teorema do homomorfismo, temos

$$
\mathbb{Z}[x] /\left\langle\Phi_{p^{n}}(x)\right\rangle \cong \mathbb{Z}[\theta] .
$$

Portanto, existe o homomorfismo de anéis $\Psi: \mathbb{Z}[\theta] \rightarrow \mathbb{Z}_{p}$. Definindo como a composta dos homomorfismo que obtemos acima, temos $\Psi(\theta)=\overline{1}$ e $\Psi$ é sobrejetor.

Seja $\psi$ a restrição de $\Psi$ a $U(\mathbb{Z}[\theta])$, isto é, $\psi:=\left.\Psi\right|_{U(\mathbb{Z}[\theta])}: U(\mathbb{Z}[\theta]) \rightarrow U\left(\mathbb{Z}_{p}\right)$. Este homomorfismo de grupos é o que realmente nos interessará. Finalizaremos esta seção mostrando somente que $\psi$ é sobrejetor.

Seja $i \in U\left(\mathbb{Z}_{p}\right)$, então $1 \leq i \leq p-1 \operatorname{com}(i, p)=1$. Portanto, $\mu_{i}=1+\theta+\cdots+\theta^{i-1} \in U(\mathbb{Z}[\theta])$ e $\psi\left(\mu_{i}\right)=i$. Logo $\psi$ é sobrejetor.

Analisaremos o núcleo deste homomorfismo na próxima seção e, a partir dele, obteremos resultados importantes desta tese.

\subsection{Primo ímpar}

Considere $I_{p^{n}}=\left\{i \in \mathbb{Z} \mid 1<i<\frac{p^{n}}{2}\right.$ e $\left.m d c\left(i, p^{n}\right)=1\right\}$ e $S_{\theta}=\left\{-1, \theta, \mu_{i} \mid i \in I_{p^{n}}\right\}$, com $\theta$ sendo uma raiz $p^{n}$-ésima primitiva da unidade. Para $p^{n}$ tal que $\phi\left(p^{n}\right) \leq 66$, temos que $S_{\theta}$ gera $U(\mathbb{Z}[\theta])$. O nosso primeiro teorema mostrará um outro conjunto gerador para $U(\mathbb{Z}[\theta])$.

Neste trabalho $\kappa$ denotará sempre o número inteiro $\frac{\phi\left(p^{n}\right)}{2}-1$, onde $\phi$ é a função de Euler.

Pelo Teorema 1.2, no caso de $p$ ser primo ímpar, $U\left(\mathbb{Z}_{p^{n}}\right)$ é um grupo cíclico. Com estes dados, temos os primeiros resultados que são extensões aos do artigo [15].

Teorema 3.1. Seja p um primo impar tal que $\phi\left(p^{n}\right) \leq 66$. Se $t \in \mathbb{Z}$ tal que $\bar{t}$ gera $U\left(\mathbb{Z}_{p^{n}}\right)$, então

$$
S_{1}=\left\{-1, \theta, \mu_{t}, \mu_{t^{2}}, \mu_{t^{3}} \cdots, \mu_{t^{\kappa}}\right\}
$$

$\operatorname{gera} U(\mathbb{Z}[\theta])$.

\section{Prova:}

Primeiramente provaremos que $S_{1} \subseteq U(\mathbb{Z}[\theta])$. Como $-1, \theta \in U(\mathbb{Z}[\theta])$, precisamos mostrar que $\mu_{t^{i}} \in U(\mathbb{Z}[\theta])$, para $1 \leq i \leq \kappa$. 
Pela Proposição 1.10, basta mostrarmos a igualdade $m d c\left(t^{i}, p^{n}\right)=1$, para $1 \leq i \leq \kappa$. Isto é válido, pois $\bar{t}$ gera $U\left(\mathbb{Z}_{p^{n}}\right)$.

Vejamos agora a outra inclusão. Pelo Corolário 1.15 , temos $U(\mathbb{Z}[\theta])=\left\langle S_{\theta}\right\rangle$ e, como $-1, \theta \in$ $U(\mathbb{Z}[\theta]) \cap\left\langle S_{1}\right\rangle$, basta provarmos que $\mu_{i} \in\left\langle S_{1}\right\rangle$, com $i \in I_{p^{n}}$.

Observe que para $i \in I_{p^{n}}$, temos $i \in U\left(\mathbb{Z}_{p^{n}}\right)=\langle\bar{t}\rangle$. Logo $i \equiv t^{j}\left(\bmod p^{n}\right)$, para algum inteiro $j$ tal que $1 \leq j \leq \phi\left(p^{n}\right)$, pois $\left|U\left(\mathbb{Z}_{p^{n}}\right)\right|=\phi\left(p^{n}\right)$. Logo $\mu_{i}=\mu_{t^{j}}$ por P5.

Seja $\mu_{i} \in U(\mathbb{Z}[\theta])$, com $i \in I_{p^{n}}$. Pela observação anterior, devemos verificar que $\mu_{i}=\mu_{t^{j}} \in\left\langle S_{1}\right\rangle$ nos seguintes casos:

1) $1 \leq j \leq \frac{\phi\left(p^{n}\right)}{2}-1$

2) $\frac{\phi\left(p^{n}\right)}{2}<j<\phi\left(p^{n}\right)$;

3) $j=\frac{\phi\left(p^{n}\right)}{2}$;

4) $j=\phi\left(p^{n}\right)$.

1) É imediato pela definição de $S_{1}$.

2) Se $\frac{\phi\left(p^{n}\right)}{2}<j<\phi\left(p^{n}\right)$, seja $r=j-\frac{\phi\left(p^{n}\right)}{2}$. Logo $t^{j} \equiv t^{r+\frac{\phi\left(p^{n}\right)}{2}}\left(\bmod p^{n}\right)$.

Afirmação: $t^{\frac{\phi\left(p^{n}\right)}{2}} \equiv-1\left(\bmod p^{n}\right)$. De fato, como $\left|U\left(\mathbb{Z}_{p^{n}}\right)\right|=\phi\left(p^{n}\right)=p^{n-1}(p-1)$ é par, existe um único subgrupo de $U\left(\mathbb{Z}_{p^{n}}\right)$ de ordem 2 , que é $A=\{-1,1\}$. Por outro lado, como $\langle\bar{t}\rangle=U\left(\mathbb{Z}_{p^{n}}\right)$, temos $\left(t, p^{n}\right)=1 \mathrm{e}$, portanto, pelo Teorema de Euler, temos $t^{\phi\left(p^{n}\right)} \equiv 1\left(\bmod p^{n}\right)$. Como $t^{\phi\left(p^{n}\right)}=\left(t^{\frac{\phi\left(p^{n}\right)}{2}}\right)^{2}$, temos $\left(t^{\frac{\phi\left(p^{n}\right)}{2}}\right)^{2} \equiv 1\left(\bmod p^{n}\right)$. No entanto, não vale $\left(t^{\frac{\phi\left(p^{n}\right)}{2}}\right) \equiv 1\left(\bmod p^{n}\right)$ já que $\bar{t}$ gera $U\left(\mathbb{Z}_{p^{n}}\right)$ e $\left|U\left(\mathbb{Z}_{p^{n}}\right)\right|=\phi\left(p^{n}\right) \geq \frac{\phi\left(p^{n}\right)}{2}$. Decorre destes fatos que $\left\{1, t^{\frac{\phi\left(p^{n}\right)}{2}}\right\}$ é um subgrupo de $U\left(\mathbb{Z}_{p^{n}}\right)$ de ordem 2 , ou seja, $A=\{-1,1\}=\left\{1, t^{\frac{\phi\left(p^{n}\right)}{2}}\right\}$. Logo $\left(t^{\frac{\phi\left(p^{n}\right)}{2}}\right) \equiv-1\left(\bmod p^{n}\right)$, o que conclui a afirmação.

Tínhamos $i \equiv t^{j} \equiv t^{r+\frac{\phi\left(p^{n}\right)}{2}}\left(\bmod p^{n}\right)$, assim, pela afirmação segue $i \equiv-t^{r}\left(\bmod p^{n}\right)$, ou seja, $i+t^{r} \equiv 0\left(\bmod p^{n}\right)$. Então $\mu_{i}=-\theta^{i} \mu_{t^{r}}$.

Como $r=j-\frac{\phi\left(p^{n}\right)}{2}$ e $\frac{\phi\left(p^{n}\right)}{2}<j<\phi\left(p^{n}\right)$, temos $1 \leq r \leq \frac{\phi\left(p^{n}\right)}{2}-1$ e daí $\mu_{t^{r}} \in S_{1}$. Logo $\mu_{i}=-\theta^{i} \mu_{t^{r}} \in\left\langle S_{1}\right\rangle$.

3) Se $j=\frac{\phi\left(p^{n}\right)}{2}$, então $i \equiv t^{\frac{\phi\left(p^{n}\right)}{2}}\left(\bmod p^{n}\right) . \operatorname{Mas} t^{\frac{\phi\left(p^{n}\right)}{2}} \equiv-1\left(\bmod p^{n}\right)$ e $-1 \equiv\left(p^{n}-1\right)\left(\bmod p^{n}\right)$, $\operatorname{logo} i \equiv\left(p^{n}-1\right)\left(\bmod p^{n}\right)$. Portanto, $\mu_{i}=\mu_{p^{n}-1}=1+\theta+\theta^{2}+\cdots+\theta^{p^{n}-2}=-\theta^{p^{n}-1} \in\left\langle S_{1}\right\rangle$.

4) Se $j=\phi\left(p^{n}\right)$, temos $i \equiv t^{\phi\left(p^{n}\right)}\left(\bmod p^{n}\right)$. Como $t^{\phi\left(p^{n}\right)} \equiv 1\left(\bmod p^{n}\right)$, concluímos que $\mu_{i}=\mu_{1}=1 \in\left\langle S_{1}\right\rangle$, pois $-1 \in\left\langle S_{1}\right\rangle$.

Deste modo mostramos que $U(\mathbb{Z}[\theta]) \subseteq\left\langle S_{1}\right\rangle$. Logo $U(\mathbb{Z}[\theta])=\left\langle S_{1}\right\rangle$. ¿ $\mathbf{x}$ 
No teorema a seguir encontramos um outro conjunto gerador de $U(\mathbb{Z}[\theta])$. A partir deste novo conjunto, tomaremos um subconjunto gerador de $\operatorname{ker}(\psi)$.

Teorema 3.2. Seja $t \in \mathbb{Z}$ tal que $\langle\bar{t}\rangle=U\left(\mathbb{Z}_{p^{n}}\right)$ e $S_{2}=\left\{-1, \theta, \mu_{t}, \mu_{t}^{-2} \mu_{t^{2}}, \mu_{t}^{-3} \mu_{t^{3}}, \cdots, \mu_{t}^{-\kappa} \mu_{t^{\kappa}}\right\}$. Então $\left\langle S_{1}\right\rangle=\left\langle S_{2}\right\rangle$.

\section{Prova:}

Claro que $\left\langle S_{2}\right\rangle \subseteq\left\langle S_{1}\right\rangle$, pois $-1, \theta, \mu_{t^{j}} \in\left\langle S_{1}\right\rangle$, para todo $1 \leq j \leq \frac{\phi\left(p^{n}\right)}{2}-1$.

Agora seja $\mu_{t^{i}} \in S_{1}$. Então $\mu_{t^{i}}=\mu_{t}^{i}\left(\mu_{t}^{-i} \mu_{t^{i}}\right)$ e como $\mu_{t}^{i}$ e $\left(\mu_{t}^{-i} \mu_{t^{i}}\right)$ são elementos de $\left\langle S_{2}\right\rangle$, segue que $\mu_{t^{i}} \in\left\langle S_{2}\right\rangle$. Portanto, $\left\langle S_{1}\right\rangle=\left\langle S_{2}\right\rangle$. is

Um fato importante a se observar é que $S_{1} \backslash\{-1, \theta\}$ e $S_{2} \backslash\{-1, \theta\}$ são $\mathbb{Z}$-bases para o complemento da parte de torção de $U(\mathbb{Z}[\theta])$, pois $S_{1}$ e $S_{2}$ geram $U(\mathbb{Z}[\theta])$ e, pelo Corolário 1.9 , o posto do complemento da parte de torção de $U(\mathbb{Z}[\theta])$ é $\kappa=\frac{\phi\left(p^{n}\right)}{2}-1$.

Façamos uma observação antes de prosseguirmos com o próximo teorema.

$t$ é um inteiro tal que $\langle\bar{t}\rangle=U\left(\mathbb{Z}_{p^{n}}\right)$, então $\langle\tilde{t}\rangle=U\left(\mathbb{Z}_{p}\right)$. De fato, seja $a \in \mathbb{Z}$ tal que $a \leq p-1$, então $a \equiv t^{i}\left(\bmod p^{n}\right)$, para algum $i \in \mathbb{N}$. Logo $a \equiv t^{i}(\bmod p)$, para todo $1 \leq a \leq p-1$. Portanto, $t$ é uma raiz primitiva módulo $p^{n}$ e $p$. Por isso, abusaremos da notação dizendo que se $t$ gera $U\left(\mathbb{Z}_{p^{n}}\right)$, então $t$ gera $U\left(\mathbb{Z}_{p}\right)$.

Definição 3.1. Seja $U\left(\mathbb{Z}_{p^{n}}\right)=\langle t\rangle$. Definimos por $\mathcal{U}$ o seguinte subconjunto de $U(\mathbb{Z}[\theta])$ :

$$
\mathcal{U}:=\left\{\theta,-\left(\mu_{t}^{\frac{p-1}{2}}\right), \mu_{t}^{-2} \mu_{t^{2}}, \cdots, \mu_{t}^{-\kappa} \mu_{t^{\kappa}}\right\}
$$

Teorema 3.3. Seja $\psi: U(\mathbb{Z}[\theta]) \rightarrow U\left(\mathbb{Z}_{p}\right)$ o homomorfismo de grupos definido na Seção 3.1. Então $\operatorname{ker}(\psi)=\langle\mathcal{U}\rangle$.

\section{Prova:}

Seja $\mu_{i}=1+\theta+\theta^{2}+\cdots+\theta^{i-1}$, então $\psi\left(\mu_{i}\right)=i \in U\left(\mathbb{Z}_{p^{n}}\right)$. Considere $t \in \mathbb{Z}$ tal que $t$ gera $U\left(\mathbb{Z}_{p^{n}}\right)$ e $U\left(\mathbb{Z}_{p}\right)$

Aplicando $\psi$ aos elementos de $\mathcal{U}$, temos:

- $\psi(\theta)=1$

- $\psi\left(\mu_{t}^{-i} \mu_{t^{i}}\right)=(t)^{-i} t^{i}=1$, para todo $2 \leq i \leq \kappa=\frac{\phi\left(p^{n}\right)}{2}-1$

- $\psi\left(-\left(\mu_{t}^{\frac{p-1}{2}}\right)\right)=-\left(t^{\frac{p-1}{2}}\right) \equiv 1(\bmod p)$, pois $t^{\frac{p-1}{2}} \equiv-1(\bmod p)$ já que $U\left(\mathbb{Z}_{p}\right)=\langle\bar{t}\rangle$. 
Logo $\langle\mathcal{U}\rangle \subseteq \operatorname{ker}(\psi)$.

Mostraremos a inclusão $\operatorname{ker}(\psi) \subseteq\langle\mathcal{U}\rangle$. Seja $v \in \operatorname{ker}(\psi) \subseteq U(\mathbb{Z}[\theta])=\left\langle S_{2}\right\rangle$. Então podemos escrever $v$ como combinação dos elementos de $S_{2}$, ou seja, $v=(-1)^{\alpha} \theta^{s_{0}} \mu_{t}^{s_{1}}\left(\mu_{t}^{-2} \mu_{t^{2}}\right)^{s_{2}} \cdots\left(\mu_{t}^{-\kappa} \mu_{t^{\kappa}}\right)^{s_{\kappa}}$, com $0 \leq \alpha \leq 1,0 \leq s_{0} \leq p^{n}-1$ e $s_{j} \in \mathbb{Z}$, para todo $j \in\{1,2, \cdots, \kappa\}$. Como $\theta,\left(\mu_{t}^{-j} \mu_{t^{j}}\right) \in \operatorname{ker}(\psi)$, então

$$
v \in \operatorname{ker}(\psi) \Leftrightarrow\left\{\begin{array}{l}
\alpha=1 \quad \text { e } t^{s_{1}} \equiv-1(\bmod p) \\
\text { ou } \\
\alpha=0
\end{array}\right.
$$

$1^{o}$ caso $\alpha=1$ e $t^{s_{1}} \equiv-1(\bmod p)$ :

Temos $t^{\frac{p-1}{2}} \equiv-1(\bmod p), \operatorname{logo} t^{s_{1}+\frac{(p-1)}{2}} \equiv 1(\bmod p)$. Como $\bar{t}$ gera $U\left(\mathbb{Z}_{p}\right)$, devemos ter $s_{1}+\frac{(p-1)}{2}=(p-1) q$, para algum $q \in \mathbb{Z}$, ou seja, $s_{1}=\frac{(p-1)}{2}(2 q-1)$. Portanto, $\mu_{t}^{s_{1}}=\left(\mu_{t}^{\frac{p-1}{2}}\right)^{2 q-1}$. Como $2 q-1$ é ímpar, então se $v \in k e r(\psi)$, temos

$$
v=(-1)^{\alpha} \theta^{s_{0}} \mu_{t}^{s_{1}}\left(\mu_{t}^{-2} \mu_{t^{2}}\right)^{s_{2}} \cdots\left(\mu_{t}^{-\kappa} \mu_{t^{\kappa}}\right)^{s_{\kappa}}=(1) \theta^{s_{0}}\left(-\mu_{t}^{\frac{p-1}{2}}\right)^{2 q-1}\left(\mu_{t}^{-2} \mu_{t^{2}}\right)^{s_{2}} \cdots\left(\mu_{t}^{-\kappa} \mu_{t^{\kappa}}\right)^{s_{\kappa}} \in\langle\mathcal{U}\rangle .
$$

$2^{o}$ caso) $\alpha=0$ e $t^{s_{1}} \equiv 1(\bmod p)$ :

Podemos considerar $s_{1}=(p-1) q=\frac{(p-1)}{2} 2 q$, para algum $q \in \mathbb{Z}$, pois $\bar{t}$ gera $U\left(\mathbb{Z}_{p}\right)$. Então, $\mu_{t}^{s_{1}}=\left(\mu_{t}^{\frac{p-1}{2}}\right)^{2 q}=\left(-\mu_{t}^{\frac{p-1}{2}}\right)^{2 q}$. Como neste caso $\alpha=0$, então

$$
v=(-1)^{\alpha} \theta^{s_{0}} \mu_{t}^{s_{1}}\left(\mu_{t}^{-2} \mu_{t^{2}}\right)^{s_{2}} \cdots\left(\mu_{t}^{-\kappa} \mu_{t^{\kappa}}\right)^{s_{\kappa}}=\theta^{s_{0}}\left(-\mu_{t}^{\frac{p-1}{2}}\right)^{2 q}\left(\mu_{t}^{-2} \mu_{t^{2}}\right)^{s_{2}} \cdots\left(\mu_{t}^{-\kappa} \mu_{t^{\kappa}}\right)^{s_{\kappa}} \in\langle\mathcal{U}\rangle .
$$

Logo $\operatorname{ker}(\psi) \subseteq\langle\mathcal{U}\rangle$, e, portanto, $\operatorname{ker}(\psi)=\langle\mathcal{U}\rangle$. ¿̇

Agora vamos mudar um pouco o conjunto $\mathcal{U}$ e obter um subgrupo de $k e r(\psi)$ de índice finito.

Observe que $-\mu_{t}^{\frac{\phi\left(p^{n}\right)}{2}} \in \mathcal{U}$, pois $-\mu_{t}^{\frac{\phi\left(p^{n}\right)}{2}}=\left(-\mu_{t}^{\frac{p-1}{2}}\right)^{p^{n-1}} \in \mathcal{U}$.

Além disso temos $t^{\frac{\phi\left(p^{n}\right)}{2}} \equiv-1\left(\bmod p^{n}\right)$, ou seja, $t^{\frac{\phi\left(p^{n}\right)}{2}} \equiv\left(p^{n}-1\right)\left(\bmod p^{n}\right)$.

Logo $\mu_{t \frac{\phi\left(p^{n}\right)}{2}}=\mu_{p^{n}-1}=-\theta^{p^{n}-1} \in \mathcal{U}$ e destas igualdades obtemos $-1=\theta \mu_{t \frac{\phi\left(p^{n}\right)}{2}}$.

Assim $-\mu_{t}^{-\frac{\phi\left(p^{n}\right)}{2}}=\theta \mu_{t} \frac{\phi\left(p^{n}\right)}{2} \mu_{t}^{-\frac{\phi\left(p^{n}\right)}{2}} \in \mathcal{U}$. Como $\theta \in \mathcal{U}$, então $\mu_{t}{ }_{\frac{\phi\left(p^{n}\right)}{2}} \mu_{t}^{-\frac{\phi\left(p^{n}\right)}{2}} \in \mathcal{U}$. Obtemos deste modo o seguinte subconjunto de $\mathcal{U}$ :

$$
\mathcal{U}^{\prime}=\left\{\theta, \mu_{t}^{-2} \mu_{t^{2}}, \cdots, \mu_{t}^{-\kappa} \mu_{t^{\kappa}}, \mu_{t}^{-(\kappa+1)} \mu_{t^{\kappa+1}}\right\}
$$


lembrando que $\kappa=\frac{\phi\left(p^{n}\right)}{2}-1$.

O conjunto $\mathcal{U}^{\prime}$, ao contrário do que ocorre quando $n=1$, não gera $k e r(\psi)$ quando trabalhamos com as potências $p^{n}$, com $n \neq 1$.

Agora, de modo análogo à definição dada por Ferraz em [15], definimos a seguinte unidade:

Definição 3.2. Dado um inteiro positivo $q$ e um inteiro $s$ relativamente primo com $p^{n}$, definimos o seguinte elemento de $\mathbb{Z}[\theta]$ :

$$
\omega_{q, s}=\sum_{j=0}^{q-1} \theta^{j s}=1+\theta^{s}+\theta^{2 s}+\cdots+\theta^{(q-1) s} .
$$

Se $q$ é positivo, então $\mu_{q}$ é exatamente a unidade $\omega_{q, 1}$. Esta definição também é válida para $p=2$.

Proposição 3.4. O elemento $\omega_{q, s}$ definido em $(* * *)$ é uma unidade de $\mathbb{Z}[\theta]$.

\section{Prova:}

Seja $j \in \mathbb{Z}$ tal que $q s j \equiv 1\left(\bmod p^{n}\right)$. Então

$$
\mu_{s} \mu_{q s}^{-1}=\left(1+\theta+\theta^{2}+\cdots+\theta^{s-1}\right)\left(1+\theta^{q s}+\theta^{2 q s}+\cdots+\theta^{q s(j-1)}\right)
$$

é o inverso de $\omega_{q, s}$. De fato,

$$
\begin{aligned}
\omega_{q, s} \mu_{s} \mu_{q s}^{-1} & =\left(1+\theta^{s}+\cdots+\theta^{(q-1) s}\right)\left(1+\theta+\theta^{2}+\cdots+\theta^{s-1}\right)\left(1+\theta^{q s}+\theta^{2 q s}+\cdots+\theta^{q s(j-1)}\right) \\
& =\left(1+\theta+\theta^{2}+\cdots+\theta^{q s-1}\right)\left(1+\theta^{q s}+\theta^{2 q s}+\cdots+\theta^{q s(j-1)}\right) \\
& =1+\theta+\theta^{2}+\cdots+\theta^{q s j-1}=1,
\end{aligned}
$$

pois $q s j \equiv 1\left(\bmod p^{n}\right)$.

Proposição 3.5. Sejam q e s inteiros positivos e $p$ um primo tais que $m d c\left(q, p^{n}\right)=1$. Então

$$
\mu_{q^{s}}=\prod_{j=0}^{s-1} \omega_{q, q^{j}}=\omega_{q, 1} \omega_{q, q} \omega_{q, q^{2}} \cdots \omega_{q, q^{s-1}}
$$

\section{Prova:}

A prova desta proposição é análoga à prova feita por Ferraz, no artigo que tomamos como base desta tese. Faremos a prova por indução em $s$. Se $s=1$, o resultado segue da definição. Vamos 
supor que o resultado vale para $s=m-1$ e provaremos que vale para $s=m$. Logo devemos provar que $\mu_{q^{m}}=\mu_{q^{m-1}} \cdot \omega_{q, q^{m-1}}$. Temos

$$
\begin{aligned}
\mu_{q^{m-1}} \cdot \omega_{q, q^{m-1}} & =\left(1+\theta+\cdots+\theta^{q^{m-1}-1}\right)\left(1+\theta^{q^{m-1}}+\cdots+\theta^{(q-1) q^{m-1}}\right)= \\
& =\left(1+\theta+\theta^{2}+\cdots+\theta^{q^{m-1}-1}\right)+\left(1+\theta+\theta^{2}+\cdots+\theta^{q^{m-1}-1}\right) \theta^{q^{m-1}}+ \\
& +\left(1+\theta+\cdots+\theta^{q^{m-1}-1}\right) \theta^{2 q^{m-1}}+\cdots+\left(1+\theta+\cdots+\theta^{q^{m-1}-1}\right) \theta^{(q-1) q^{m-1}}= \\
& =\left(1+\theta+\theta^{2}+\cdots+\theta^{q^{m-1}-1}\right)+\left(\theta^{q^{m-1}}+\theta^{q^{m-1}+1} \cdots+\theta^{2 q^{m-1}-1}\right)+ \\
& +\left(\theta^{2 q^{m-1}}+\cdots+\theta^{3 q^{m-1}-1}\right)+\cdots+\left(\theta^{(q-1) q^{m-1}}+\cdots+\theta^{q q^{m-1}-1}\right)= \\
& =1+\theta+\theta^{2}+\cdots+\theta^{q^{m}-1}=\mu_{q^{m}}
\end{aligned}
$$

$\operatorname{Logo} \mu_{q^{m}}=\mu_{q^{m-1}} \cdot \omega_{q, q^{m-1}} . \mathbf{z}$

Com a unidade definida anteriormente iremos definir agora uma outra unidade de $U(\mathbb{Z}[\theta])$, que será a chave para encontrarmos as unidades de $\mathbb{Z} C_{p^{n}}$.

Definição 3.3. Seja $t$ um inteiro fixo tal que $t$ é gerador de $U\left(\mathbb{Z}_{p}\right)$ e de $U\left(\mathbb{Z}_{p^{n}}\right)$. Definimos $h_{i}$ como sendo a seguinte unidade de $U(\mathbb{Z}[\theta])$ :

$$
h_{i}=\omega_{t, 1}^{-1} \omega_{t, t^{i}}
$$

Teorema 3.6. Seja $\mathcal{U}_{0}=\left\{\theta, h_{1}, h_{2}, \cdots, h_{\kappa}\right\}$, com $\kappa=\frac{\phi\left(p^{n}\right)}{2}-1$. Então $\mathcal{U}_{0}$ gera $\left\langle\mathcal{U}^{\prime}\right\rangle$, onde $\mathcal{U}^{\prime}=\left\{\theta, \mu_{t}^{-2} \mu_{t^{2}}, \cdots, \mu_{t}^{-(\kappa+1)} \mu_{t^{\kappa+1}}\right\}$.

\section{Prova:}

Pela proposição anterior, temos

$$
\mu_{t^{i}}=\omega_{t, 1} \omega_{t, t} \omega_{t, t^{2}} \cdots \omega_{t, t^{i-1}}=\mu_{t} \omega_{t, t} \omega_{t, t^{2}} \cdots \omega_{t, t^{i-1}}
$$

ou seja, $\mu_{t^{i}}=\mu_{t} \omega_{t, t} \omega_{t, t^{2}} \cdots \omega_{t, t^{i-1}}$ e, multiplicando os dois lados desta igualdade por $\mu_{t}^{-i}$, temos

$$
\begin{aligned}
\mu_{t}^{-i} \mu_{t^{i}} & =\mu_{t}^{-i+1}\left(\omega_{t, t} \omega_{t, t^{2}} \cdots \omega_{t, t^{i-1}}\right) \\
& =\left(\mu_{t}^{-1} \omega_{t, t}\right)\left(\mu_{t}^{-1} \omega_{t, t^{2}}\right) \cdots\left(\mu_{t}^{-1} \omega_{t, t^{i-1}}\right) \\
& =h_{1} h_{2} \cdots h_{i-1} \in\left\langle\mathcal{U}_{0}\right\rangle
\end{aligned}
$$


para todo $i \in \mathbb{Z}$, com $2 \leq i \leq \kappa+1$. Logo $\mathcal{U}^{\prime} \subseteq\left\langle\mathcal{U}_{0}\right\rangle$.

Agora vamos mostrar a outra inclusão. Novamente pela proposição anterior temos

$$
\mu_{t^{i+1}}=\underbrace{\omega_{t, 1} \omega_{t, t} \omega_{t, t^{2}} \cdots \omega_{t, t^{i-1}}}_{\mu_{t^{i}}} \omega_{t, t^{i}}=\mu_{t^{i}} \omega_{t, t^{i}}
$$

Desta igualdade decorre que $\omega_{t, t^{i}}=\mu_{t^{i+1}} \mu_{t^{i}}^{-1}$. Logo

$$
\begin{aligned}
h_{i} & =\omega_{t, 1}^{-1} \omega_{t, t^{i}}=\omega_{t, 1}^{-1} \mu_{t^{i+1}} \mu_{t^{i}}^{-1}=\mu_{t}^{-1} \mu_{t}^{i} \mu_{t}^{-i} \mu_{t^{i+1}} \mu_{t^{i}}^{-1} \\
& =\left(\mu_{t}^{-(i+1)} \mu_{t^{i+1}}\right)\left(\mu_{t}^{-i} \mu_{t^{i}}\right)^{-1} \in\left\langle\mathcal{U}^{\prime}\right\rangle .
\end{aligned}
$$

Portanto, $h_{i} \in\left\langle\mathcal{U}^{\prime}\right\rangle$, para todo inteiro $i, 1 \leq i \leq \kappa$. Logo $\mathcal{U}_{0} \subseteq\left\langle\mathcal{U}^{\prime}\right\rangle$ e provamos o que queríamos. $\left.\boldsymbol{\star}\right\rangle$

\subsection{Primo 2}

Nesta seção iremos estudar o caso $p=2$ da condição $\phi\left(p^{n}\right) \leq 66$. O inteiro positivo $n$ das potências $2^{n}$ que satisfazem esta condição são 1,2,3,4,5,6 e 7. Excluiremos os casos 1, 2 e 3, pois os dois primeiros são os casos em que $U\left(\mathbb{Z} C_{2^{n}}\right)$ é trivial e $n=3$ pode ser encontrada no livro $A n$ Introduction to Group Rings dos autores C.Polcino Milies e S. K. Sehgal ou no livro Commutative Group Algebras de G. Karpilovsky.

No caso em que $p$ é um primo ímpar, temos que $U\left(\mathbb{Z}_{p^{n}}\right)$ é um grupo cíclico, mas quando $p=2$ vale o seguinte:

$$
U\left(\mathbb{Z}_{p^{n}}\right)=\left\langle 2^{n}-1\right\rangle \times\langle 3\rangle \cong C_{2} \times C_{2^{n-2}} .
$$

Os resultados nesta seção são bem parecidos com o da seção anterior, mas como $U\left(\mathbb{Z}_{2^{n}}\right)$ não é cíclico, fizemos modificações. Aqui $\theta$ é uma raiz primitiva $2^{n}$-ésima da unidade.

Inicialmente vejamos uma propriedade da Teoria dos Números a respeito das potências de 2 .

P7. Seja $n$ um número inteiro maior que 2 , então $a^{\frac{\phi\left(2^{n}\right)}{2}}=a^{2^{n-2}} \equiv 1\left(\bmod 2^{n}\right)$, para qualquer número ímpar $a$. 


\section{Prova:}

Vamos provar por indução. Como $a$ é ímpar, considere $a=2 x+1$. Para $n=3$, temos

$$
a^{2^{3-2}}=a^{2}=(2 x+1)^{2}=4 x^{2}+4 x+1=4 x(x+1)+1
$$

Como ou $x$ ou $x+1$ é par, segue que $4 x(x+1)=8 q$, para algum $q \in \mathbb{Z}$ e dái $a^{2^{3-2}}=8 q+1 \equiv$ $1\left(\bmod 2^{3}\right)$.

Agora suponha que a propriedade vale para $n=k$ e provaremos que vale para $n=k+1$. Como $a$ é ímpar, segue que $a^{2^{k-2}}+1=2 q$, para algum $q \in \mathbb{Z}$, ou seja, $a^{2^{k-2}}+1$ é par. Pela hipótese de indução tem-se $a^{2^{k-2}}-1=2^{k} m$, para algum $m \in \mathbb{Z}$. Logo

$$
\left(a^{2^{k+1-2}}-1\right)=\left(a^{2^{k-2}}-1\right)\left(a^{2^{k-2}}+1\right)=2^{k} m 2 q=2^{k+1} m q
$$

e, portanto, $a^{2^{k+1-2}} \equiv 1\left(\bmod 2^{k+1}\right)$.

Logo a propriedade vale para todo $n \in \mathbb{N}$. is

Proposição 3.7. Sejam $\theta$ uma raiz primitiva $2^{n}$-ésima da unidade e $i=\left(2^{n}-1\right)^{s} 3^{q}$, com $0 \leq s \leq 1$ e $0 \leq q \leq 2^{n-2}-1$. Então são válidos os seguintes itens:

(i) $s=0 \Longrightarrow \mu_{i}=\mu_{3 q}$;

(ii) $s=1 \Longrightarrow \mu_{i}=-\theta^{i} \mu_{3^{q}}$

\section{Prova:}

A prova de (i) é trivial. Verifiquemos (ii).

Como $s=1$ e $q \neq 0$, então $1 \leq i=\left(2^{n}-1\right) 3^{q} \leq 2^{n} 3^{q}$. Temos ainda $2^{n} 3^{q}=i+3^{q}$, portanto, $i+3^{q} \equiv 0\left(\bmod 2^{n}\right)$ e pela propriedade $(\mathbf{P} 4)$ segue $\mu_{i}=-\theta^{i} \mu_{3^{q}}$. ¿ $\mathbf{z}$

$\operatorname{Temos} U(\mathbb{Z}[\theta])=\left\langle\left\{\theta, \mu_{i} \mid i \in \mathcal{I}_{2^{n}}\right\}\right\rangle$, onde $\mathcal{I}_{2^{n}}=\left\{i \in \mathbb{Z} \mid 1<i<2^{n-1}\right.$ e $\left.m d c\left(i, 2^{n}\right)=1\right\}$. Da mesma maneira que na seção anterior, iremos exibir um novo conjunto gerador para $U(\mathbb{Z}[\theta])$.

Teorema 3.8. Seja $3<n \in \mathbb{Z}$ tal que $\phi\left(2^{n}\right) \leq 66$. Seja $S_{1}=\left\{\theta, \mu_{3}, \mu_{3^{2}}, \cdots, \mu_{3^{\kappa}}\right\}$, onde $\kappa=\frac{\phi\left(2^{n}\right)}{2}-1$. Então

$$
\left\langle S_{1}\right\rangle=U(\mathbb{Z}[\theta])
$$




\section{Prova:}

Primeiramente provaremos a inclusão $S_{1} \subseteq U(\mathbb{Z}[\theta])$. Como $\theta \in U(\mathbb{Z}[\theta])$, analisaremos $\mu_{3^{q}}$ nos seguintes casos:

1) $3^{q}=1$;

2) $1<3^{q}<2^{n-1}$;

3) $2^{n-1}<3^{q}<2^{n}$;

4) $3^{q}>2^{n}$.

1) $\mu_{3^{q}}=\mu_{1}=1$, por definição.

2) Se $1<3^{q}<2^{n-1}$, então $3^{q} \in \mathcal{I}_{2^{n}}$ e , portanto, $\mu_{3^{q}} \in U(\mathbb{Z}[\theta])$.

3) Se $2^{n-1}<3^{q}<2^{n}$, então $2^{n}-2^{n-1}=2^{n-1}>2^{n}-3^{q}>0$. Temos ainda $m d c\left(2^{n}-3^{q}, 2^{n}\right)=1$. Assim se $2^{n}-3^{q} \neq 1$, então $2^{n}-3^{q} \in \mathcal{I}_{2^{n}}$. Daí $\mu_{2^{n}-3^{q}} \in U(\mathbb{Z}[\theta])$. Como $\theta \in U(\mathbb{Z}[\theta])$, temos $\mu_{3^{q}}=$ $-\theta^{3^{q}} \mu_{2^{n}-3^{q}} \in U(\mathbb{Z}[\theta])$. Caso $2^{n}-3^{q}=1$, então $3^{q}=2^{n}-1$ e daí decorre $\mu_{3^{q}}=-\theta^{2^{n}-1} \in U(\mathbb{Z}[\theta])$.

4) Se $3^{q}>2^{n}$, então existe $x \in \mathbb{Z} \operatorname{com} 0<x<2^{n}$ tal que $3^{q} \equiv x\left(\bmod 2^{n}\right)$. Então $x$ recai num dos casos anteriores, ou seja, $x=1,1<x<2^{n-1}$ ou $2^{n-1}<x<2^{n}$. Logo $\mu_{3^{q}}=\mu_{x} \in U(\mathbb{Z}[\theta])$.

Observe que $m d c\left(x, 2^{n}\right)=1$, pois $3^{q} \equiv x\left(\bmod 2^{n}\right)$ e $3^{q}$ é ímpar. Portanto, $S_{1} \subseteq U(\mathbb{Z}[\theta])$.

Na outra inclusão, como $U(\mathbb{Z}[\theta])=\left\langle\left\{-1, \theta, \mu_{i} \mid i \in \mathcal{I}_{2^{n}}\right\}\right\rangle$, basta verificar que $\mu_{i} \in\left\langle S_{1}\right\rangle$, para $i \in \mathcal{I}_{2^{n}}$.

Se $i \in \mathcal{I}_{2^{n}}$, então $i \in U\left(\mathbb{Z}_{2^{n}}\right)=\left\langle 2^{n}-1\right\rangle \times\langle 3\rangle$. Assim, $i=\left(2^{n}-1\right)^{s} 3^{q}$, com $0 \leq s \leq 1 \mathrm{e}$ $0 \leq q \leq 2^{n-2}-1$. Neste caso, pela Proposição $3.7, \mu_{i}=\mu_{3^{q}} \in S_{1}$ ou $\mu_{i}=-\theta^{i} \mu_{3^{q}} \in\left\langle S_{1}\right\rangle$. Logo $\mu_{i} \in\left\langle S_{1}\right\rangle$, para todo $i \in \mathcal{I}_{2^{n}}$. \&

Observe que $S_{1} \backslash\{\theta\}$ é um conjunto multiplicativamente independente, pois o posto da parte livre de torção de $U(\mathbb{Z}[\theta])$ é $\kappa=\frac{\phi\left(2^{n}\right)}{2}-1$ e $U(\mathbb{Z}[\theta])=\left\langle S_{1}\right\rangle$.

Exemplo 3.1. Consideremos o caso $p^{n}=16$. Sabemos que $\mu_{3}, \mu_{5}$ e $\mu_{7}$ formam um sistema fundamental de $U(\mathbb{Z}[\theta])$ e $S_{1}=\left\{-1, \theta, \mu_{3}, \mu_{3^{2}}, \mu_{3^{3}}\right\}$. Temos então:

$\mu_{3^{2}}=\mu_{9}=-\theta^{9} \mu_{16-9}=-\theta^{9} \mu_{7} \Rightarrow \mu_{7}=-\theta^{7} \mu_{3^{2}} \in\left\langle S_{1}\right\rangle$;

$3^{3} \equiv 11(\bmod 16) \Rightarrow \mu_{3^{3}}=\mu_{11}=-\theta^{11} \mu_{16-11}=-\theta^{11} \mu_{5} \Rightarrow \mu_{5}=-\theta^{5} \mu_{3^{3}} \in\left\langle S_{1}\right\rangle$.

Como $\theta$ e $\mu_{3}$ pertencem a $S_{1}$, temos $U(\mathbb{Z}[\theta])=\left\langle S_{1}\right\rangle$.

Vamos agora encontrar um outro gerador de $U(\mathbb{Z}[\theta])$. 
Teorema 3.9. Sejam $3<n \in \mathbb{Z}$ tal que $\phi\left(2^{n}\right) \leq 66$ e $S_{2}=\left\{\theta, \mu_{3}, \mu_{3}^{-2} \mu_{3^{2}}, \mu_{3}^{-3} \mu_{3^{3}}, \cdots, \mu_{3}^{-\kappa} \mu_{3^{\kappa}}\right\}$, onde $\kappa=\frac{\phi\left(2^{n}\right)}{2}-1$. Então

$$
\left\langle S_{2}\right\rangle=\left\langle S_{1}\right\rangle
$$

\section{Prova:}

Como $\mu_{3^{i}} \in S_{1}$, para todo $1 \leq i \leq \kappa$, temos $\mu_{3}^{-i} \mu_{3^{i}} \in\left\langle S_{1}\right\rangle$. Ainda $-1, \theta \in\left\langle S_{1}\right\rangle \cap\left\langle S_{2}\right\rangle$. Logo $S_{2} \subseteq\left\langle S_{1}\right\rangle$.

Agora seja $\mu_{3^{i}} \in S_{1}$, com $1 \leq i \leq \kappa$. Temos $\mu_{3^{i}}=\mu_{3}^{i}\left(\mu_{3}^{-i} \mu_{3^{i}}\right) \in\left\langle S_{2}\right\rangle$, pois $\mu_{3}^{i}, \mu_{3}^{-i} \mu_{3^{i}} \in S_{2}$.

Portanto, $\left\langle S_{2}\right\rangle=\left\langle S_{1}\right\rangle$. is

No final da Seção 1 deste capítulo vimos que o homomorfismo de grupos $\psi: U(\mathbb{Z}[\theta]) \rightarrow U\left(\mathbb{Z}_{p}\right)$ é sobrejetor. Quando $p=2$, é claro que $U(\mathbb{Z}[\theta])=\operatorname{ker}(\psi)$ já que $\operatorname{Im}(\psi)=U\left(\mathbb{Z}_{2}\right)=\{1\}$. Logo temos as igualdades:

$$
U(\mathbb{Z}[\theta])=\operatorname{ker}(\psi)=\left\langle S_{1}\right\rangle=\left\langle S_{2}\right\rangle
$$

Proposição 3.10. Seja $\mathcal{U}^{\prime}=\left\{\theta, \mu_{3}^{-2} \mu_{3^{2}}, \mu_{3}^{-3} \mu_{3^{3}}, \cdots, \mu_{3}^{-\kappa} \mu_{3^{\kappa}}, \mu_{3}^{-(\kappa+1)}\right\}$ um subconjunto de $U(\mathbb{Z}[\theta])$. Então $\left\langle\mathcal{U}^{\prime}\right\rangle$ é um subgrupo de $U(\mathbb{Z}[\theta])$ de índice $\kappa+1=\frac{\phi\left(2^{n}\right)}{2}=2^{n-2}$.

\section{Prova:}

Observe que $2^{n-2}=\frac{\phi\left(2^{n}\right)}{2}=\kappa+1$ e por $(\mathbf{P} 7)$ temos $3^{\kappa+1} \equiv 1\left(\bmod 2^{n}\right)$. Então $\mu_{3^{\kappa+1}}=\mu_{1}=1 \mathrm{e}$, portanto, $\mu_{3}^{-(\kappa+1)} \mu_{3^{\kappa+1}}=\mu_{3}^{-(\kappa+1)}$. Como $U(\mathbb{Z}[\theta])=\left\langle S_{2}\right\rangle=\left\langle\left\{\theta, \mu_{3}, \mu_{3}^{-2} \mu_{3^{2}}, \mu_{3}^{-3} \mu_{3^{3}} \cdots, \mu_{3}^{-\kappa} \mu_{3^{\kappa}}\right\}\right\rangle$, então $\left|U_{1}(\mathbb{Z}[\theta]):\left\langle\mathcal{U}^{\prime}\right\rangle\right|=\kappa+1=\frac{\phi\left(2^{n}\right)}{2}$. is

Agora vamos utilizar uma unidade que foi definida na seção anterior,

$$
\omega_{q, s}=1+\theta^{s}+\theta^{2 s}+\cdots+\theta^{(q-1) s},
$$

para definir a seguinte unidade de $U(\mathbb{Z}[\theta])$ :

$$
h_{i}=\omega_{3,1}^{-1} \omega_{3,3^{i}}, \quad 1 \leq i \leq \kappa .
$$

Esta unidade será importante para posteriormente encontrarmos as unidades de $\mathbb{Z} C_{p^{n}}$. 
Teorema 3.11. Com a notação anterior, o conjunto $\mathcal{U}_{0}=\left\{\theta, h_{1}, h_{2}, \cdots, h_{\kappa}\right\}$ gera $\left\langle\mathcal{U}^{\prime}\right\rangle$.

\section{Prova:}

A prova deste teorema é análoga à prova do Teorema 3.6, substituindo $t$ por 3 . ¿s

É claro que $\mathcal{U}_{0} \backslash\{\theta\}$ é um conjunto multiplicativamente independente.

Para finalizar este capítulo, vejamos um exemplo:

Exemplo 3.2. Façamos novamente o exemplo para o caso $p^{n}=16$. Neste caso temos $\kappa=3$ e:

$$
\begin{aligned}
h_{1}=\omega_{3,1}^{-1} \omega_{3,3} & =\left(1+\theta+\theta^{2}\right)^{-1}\left(1+\theta^{3}+\theta^{2 \cdot 3}\right) \\
& =-\theta\left(1+\theta^{3}+\theta^{6}+\theta^{9}+\theta^{12}\right)\left(1+\theta^{3}+\theta^{6}\right) ; \\
h_{2}=\omega_{3,1}^{-1} \omega_{3,3^{2}} & =\left(1+\theta+\theta^{2}\right)^{-1}\left(1+\theta^{3^{2}}+\theta^{2 \cdot 3^{2}}\right) \\
& =-\theta\left(1+\theta^{3}+\theta^{6}+\theta^{9}+\theta^{12}\right)\left(1+\theta^{9}+\theta^{2}\right) ; \\
h_{3}=\omega_{3,1}^{-1} \omega_{3,3^{3}} & =\left(1+\theta+\theta^{2}\right)^{-1}\left(1+\theta^{3^{3}}+\theta^{2 \cdot 3^{3}}\right) \\
& =-\theta\left(1+\theta^{3}+\theta^{6}+\theta^{9}+\theta^{12}\right)\left(1+\theta^{11}+\theta^{6}\right) .
\end{aligned}
$$

Logo $\mathcal{U}_{0}=\left\{-\theta\left(1+\theta^{3}+\theta^{6}+\theta^{9}+\theta^{12}\right)\left(1+\theta^{3}+\theta^{6}\right),-\theta\left(1+\theta^{3}+\theta^{6}+\theta^{9}+\theta^{12}\right)\left(1+\theta^{2}+\theta^{9}\right)\right.$, $\left.-\theta\left(1+\theta^{3}+\theta^{6}+\theta^{9}+\theta^{12}\right)\left(1+\theta^{6}+\theta^{11}\right)\right\}$. 


\section{Capítulo 4}

\section{Unidades de $\mathbb{Z} C_{p^{n}}$}

Neste capítulo iremos encontrar um conjunto de geradores independentes de $U\left(\mathbb{Z} C_{p^{n}}\right)$ nas mesmas condições do capítulo anterior, ou seja, quando o conjunto $\left\{ \pm \theta, \mu_{t} \mid 1<t<p^{n} / 2, m d c\left(p^{n}, t\right)=\right.$ $1\}$ gera $U(\mathbb{Z}[\theta])$. Na verdade, neste capítulo, restringiremos aos primos $p$ tais que $\phi\left(p^{n}\right) \leq 66$. Esta restrição se deve ao Teorema 1.15, que nos dá uma condição suficiente para encontrarmos o sistema fundamental de $U(\mathbb{Z}[\theta])$. No entanto, esta não é uma condição necessária, podendo esta quantidade de números primos aumentar.

No capítulo anterior estendemos os resultados do artigo de Ferraz [15] mas, como no caso em que $n \geq 2$, nem sempre temos o isomorfismo entre $U_{1}\left(\mathbb{Z} C_{p^{n}}\right)$ e $k e r(\psi)$ como no caso $n=1$, encontramos um outro complemento para a parte de torção de $U_{1}\left(\mathbb{Z} C_{p^{n}}\right)$.

Usaremos as seguintes notações:

$C_{p^{n}}=\langle g\rangle$ e $C_{p^{n-1}}=\langle h\rangle$ serão grupos cíclicos de ordem $p^{n}$ e $p^{n-1}$, respectivamente, e $x=h-1$.

$\pi_{1}: \mathbb{Z} C_{p^{n}} \rightarrow \mathbb{Z}[\theta]$ e $\pi_{2}: \mathbb{Z} C_{p^{n}} \rightarrow \mathbb{Z} C_{p^{n-1}}$ denotarão os homomorfismos de anéis definidos por $\pi_{1}(g)=\theta$ e $\pi_{2}(g)=h$.

Alguns autores denotam por $U_{1}(\mathbb{Z}[\theta])$ o conjunto $\left\{\sum_{i=0}^{p^{n}-1} a_{i} \theta^{i} \mid \sum_{i=0}^{p^{n}-1} a_{i} \equiv 1(\bmod p)\right\}$, que é o núcleo do homomorfismo $\psi: U(\mathbb{Z}[\theta]) \rightarrow U\left(\mathbb{Z}_{p}\right)$. Assim utilizaremos $U_{1}(\mathbb{Z}[\theta])$ para indicar $k e r(\psi)$.

Sendo $\pi_{1}$ o homomorfismo de anéis usual que citamos acima, denotaremos por $\bar{\pi}_{1}$ o seguinte homomorfismo de grupos:

$$
\begin{array}{ccc}
\bar{\pi}_{1}: U_{1}\left(\mathbb{Z} C_{p^{n}}\right) & \longrightarrow & U_{1}(\mathbb{Z}[\theta]) \\
u & \longmapsto & \bar{\pi}_{1}(u)=\pi_{1}(u)
\end{array}
$$

Dividiremos este capítulo novamente em 3 seções. Primeiramente iremos decompor $U_{1}\left(\mathbb{Z} C_{p^{n}}\right)$ 
no seguinte produto direto:

$$
U_{1}\left(\mathbb{Z} C_{p^{n}}\right) \cong \operatorname{ker}\left(\bar{\pi}_{1}\right) \times \operatorname{Im}\left(\bar{\pi}_{1}\right)
$$

Na segunda seção encontramos os geradores independentes de $\operatorname{ker}\left(\bar{\pi}_{1}\right)$ e na terceira mostramos a validade de um importante resultado. Ressaltamos que nas duas últimas seções os resultados foram encontrados caso a caso, para cada uma das potências $p^{n}$ tal que $\phi\left(p^{n}\right) \leq 66$.

\subsection{Uma caracterização para $U_{1}\left(\mathbb{Z} C_{p^{n}}\right)$}

Vejamos agora quais relações existem entre a álgebra de grupo $\mathbb{Z} C_{p^{n}}$ e o anel dos inteiros $\mathbb{Z}[\theta]$, onde $\theta$ é uma raiz $p^{n}$-ésima primitiva da unidade. Em todos os teoremas e proposições a seguir, estaremos considerando $p$ um número primo tal que $\phi\left(p^{n}\right) \leq 66$, isto quando não citado na hipótese.

Proposição 4.1. Seja $\mathcal{U}_{0}=\left\{\theta, h_{1}, h_{2}, \cdots, h_{\kappa}\right\}$, onde $h_{i}$ é a unidade de $\mathbb{Z}[\theta]$ definida no capítulo anterior por

$$
h_{i}=\omega_{t, 1}^{-1} \omega_{t, t^{i}}=\left(1+\theta+\theta^{2}+\cdots+\theta^{t-1}\right)^{-1}\left(1+\theta^{t^{i}}+\theta^{2 t^{i}}+\cdots+\theta^{(t-1) t^{i}}\right),
$$

onde $t \in \mathbb{Z}$ é tal que $\bar{t}$ gera $U\left(\mathbb{Z}_{p}\right)$, se p é primo impar, e $t=3$, se $p=2$ e $\kappa=\frac{\phi\left(p^{n}\right)}{2}-1$. Então $\left\langle\mathcal{U}_{0}\right\rangle \subseteq \operatorname{Im}\left(\bar{\pi}_{1}\right)$.

\section{Prova:}

Claro que $\theta \in \operatorname{Im}\left(\bar{\pi}_{1}\right)$. Vejamos então que $h_{i} \in \operatorname{Im}\left(\bar{\pi}_{1}\right), 1 \leq i \leq \kappa$. Temos

$$
\begin{aligned}
h_{i} & =\omega_{t, 1}^{-1} \omega_{t, t^{i}}=\left(1+\theta+\theta^{2}+\cdots+\theta^{t-1}\right)^{-1}\left(1+\theta^{t^{i}}+\theta^{2 t^{i}}+\cdots+\theta^{(t-1) t^{i}}\right) \\
& =\left(1+\theta^{t}+\theta^{2 t}+\cdots+\theta^{(r-1) t}\right)\left(1+\theta^{t^{i}}+\theta^{2 t^{i}}+\cdots+\theta^{(t-1) t^{i}}\right),
\end{aligned}
$$

onde $t$ é um inteiro nas condições da hipótese e $r$ é o menor inteiro positivo tal que $t r \equiv 1\left(\bmod p^{n}\right)$.

Observe ainda que

$\vartheta_{i}=\left(1+g^{t}+g^{2 t}+\cdots+g^{(r-1) t}\right)\left(1+g^{t^{i}}+g^{2 t^{i}}+\cdots+g^{(t-1) t^{i}}\right)-\frac{(t r-1)}{p^{n}}\left(1+g+g^{2}+\cdots+g^{p^{n}-1}\right) \in U_{1}\left(\mathbb{Z} C_{p^{n}}\right)$,

pois esta é exatamente uma unidade de Hoechsmann.

Como $\bar{\pi}_{1}\left(\vartheta_{i}\right)=h_{i}$, então $\mathcal{U}_{0} \subseteq \operatorname{Im}\left(\bar{\pi}_{1}\right)$. ¿ t 
De agora em diante, $t$ será sempre um inteiro nas condições da Proposição 4.1:

1) Se $p$ é ímpar, então $t \in \mathbb{Z}$ tal que $\bar{t}$ gera $U\left(\mathbb{Z}_{p^{n}}\right)$;

2) Se $p=2$, então $t=3$.

E $\kappa$ denotará sempre o inteiro $\kappa=\frac{\phi\left(p^{n}\right)}{2}-1$, com $n \geq 2$.

Pelos teoremas 3.6 e 3.11 os conjuntos $\mathcal{U}_{0}=\left\{\theta, h_{1}, h_{2}, \cdots, \quad h_{\kappa}\right\} \quad$ e $\mathcal{U}^{\prime}=\left\{\theta, \mu_{t}^{-2} \mu_{t^{2}}, \cdots, \mu_{t}^{-(\kappa+1)} \mu_{t^{\kappa+1}}\right\}$ geram o mesmo subgrupo, portanto, tem-se também $\left\langle\mathcal{U}^{\prime}\right\rangle \subseteq \operatorname{Im}\left(\bar{\pi}_{1}\right)$. Segue deste fato, o seguinte lema:

Proposição 4.2. Se $(-1)^{p}\left(\mu_{t}\right)^{\frac{\kappa+1}{p}}=(-1)^{p}\left(\mu_{t}\right)^{\frac{\phi\left(p^{n}\right)}{2 p}} \notin \operatorname{Im}\left(\bar{\pi}_{1}\right)$, então

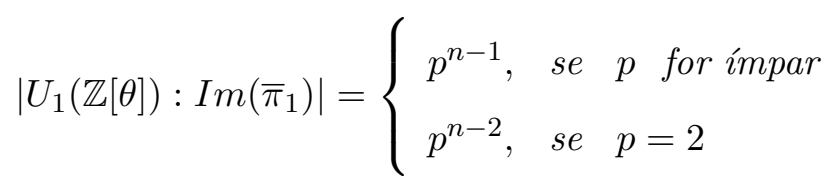

Mais ainda, $\operatorname{Im}\left(\bar{\pi}_{1}\right)=\left\langle\mathcal{U}_{0}\right\rangle$.

\section{Prova:}

Pela Proposição 4.1 e pela hipótese, temos a seguinte cadeia:

$$
\left\langle\mathcal{U}_{0}\right\rangle \subseteq \operatorname{Im}\left(\bar{\pi}_{1}\right) \subsetneq U_{1}(\mathbb{Z}[\theta])
$$

Quando $p$ é ímpar temos

$$
U_{1}(\mathbb{Z}[\theta])=\left\langle\left\{\theta,-\left(\mu_{t}^{\frac{p-1}{2}}\right), \mu_{t}^{-2} \mu_{t^{2}}, \cdots, \mu_{t}^{-\kappa} \mu_{t^{\kappa}}\right\}\right\rangle
$$

e

$$
\left\langle\mathcal{U}^{\prime}\right\rangle=\left\langle\left\{\theta, \mu_{t}^{-2} \mu_{t^{2}}, \cdots, \mu_{t}^{-(\kappa+1)} \mu_{t^{\kappa+1}}\right\}\right\rangle=\left\langle\mathcal{U}_{0}\right\rangle
$$

Como $\mu_{t}^{-(\kappa+1)} \mu_{t^{\kappa+1}}=\theta^{-1} \cdot\left(-\mu_{t}^{-\frac{p-1}{2}}\right)^{p^{n-1}}$ e $\theta^{-1}$ pertencem a $\operatorname{Im}\left(\bar{\pi}_{1}\right)$, então $\left(-\mu_{t}^{-\frac{p-1}{2}}\right)^{p^{n-1}} \in \operatorname{Im}\left(\bar{\pi}_{1}\right)$. Por hipótese, $(-1)^{p}\left(\mu_{t}\right)^{\frac{\kappa+1}{p}}=(-1)\left(\mu_{t}\right)^{\frac{(p-1)}{2} p^{n-2}} \notin \operatorname{Im}\left(\bar{\pi}_{1}\right)$, assim $\left|U_{1}(\mathbb{Z}[\theta]): \operatorname{Im}\left(\bar{\pi}_{1}\right)\right|=p^{n-1}$.

Como $\left|U_{1}(\mathbb{Z}[\theta]): \mathcal{U}_{0}\right|=p^{n-1}$ temos $\left|\operatorname{Im}\left(\bar{\pi}_{1}\right):\left\langle\mathcal{U}_{0}\right\rangle\right|=1$ e, portanto, $\operatorname{Im}\left(\bar{\pi}_{1}\right)=\left\langle\mathcal{U}_{0}\right\rangle$.

Quando $p=2$, pela Proposição 3.10, $\left|U_{1}(\mathbb{Z}[\theta]):\left\langle\mathcal{U}^{\prime}\right\rangle\right|=\left|U_{1}(\mathbb{Z}[\theta]):\left\langle\mathcal{U}_{0}\right\rangle\right|=$ $=\kappa+1=\frac{\phi\left(2^{n}\right)}{2}=2^{n-2}=p^{n-2}$. Como $\mu_{3}^{2^{n-2}}=\left(\mu_{3^{\kappa+1}} \mu_{3}^{-(\kappa+1)}\right)^{-1} \in \operatorname{Im}\left(\bar{\pi}_{1}\right)$ e, por hipótese, $\mu_{3}^{2^{n-3}} \notin \operatorname{Im}\left(\bar{\pi}_{1}\right)$, temos $\left|U_{1}(\mathbb{Z}[\theta]): \operatorname{Im}\left(\bar{\pi}_{1}\right)\right|=2^{n-2}, \operatorname{logo} \operatorname{Im}\left(\bar{\pi}_{1}\right)=\left\langle\mathcal{U}_{0}\right\rangle$. ¿ 
Provaremos mais adiante que para $p^{n}$ tal que $\phi\left(p^{n}\right) \leq 66$, a hipótese da proposição anterior é verdadeira. O resultado a seguir é muito importante no desenvolvimento do nosso trabalho, pois encontraremos um isomorfismo entre $\operatorname{Im}\left(\bar{\pi}_{1}\right)=\left\langle\mathcal{U}_{0}\right\rangle$ e um subgrupo de $U_{1}\left(\mathbb{Z} C_{p^{n}}\right)$ e veremos que este isomorfismo é que nos possibilitou encontrar os geradores de $U_{1}\left(\mathbb{Z} C_{p^{n}}\right)$.

Teorema 4.3. Seja $S=\left\{g, \vartheta_{1}, \vartheta_{2}, \vartheta_{3}, \cdots, \vartheta_{\kappa}\right\}$ um subconjunto de $U_{1}\left(\mathbb{Z} C_{p^{n}}\right)$, onde $\vartheta_{i}=\left(1+g^{t}+g^{2 t}+\cdots+g^{(r-1) t}\right)\left(1+g^{t^{i}}+g^{2 t^{i}}+\cdots+g^{(t-1) t^{i}}\right)-\frac{(t r-1)}{p^{n}}\left(1+g+g^{2}+\cdots+g^{p^{n}-1}\right) \in U_{1}\left(\mathbb{Z} C_{p^{n}}\right)$ é a unidade de Hoechsmann e suponha $(-1)^{p}\left(\mu_{t}\right)^{\frac{\phi\left(p^{n}\right)}{2 p}} \notin \operatorname{Im}\left(\bar{\pi}_{1}\right)$. Então vale o seguinte isomorfismo de grupos multiplicativos:

$$
\langle S\rangle \cong\left\langle\mathcal{U}_{0}\right\rangle
$$

\section{Prova:}

Temos o homomorfismo sobrejetor $\bar{\pi}_{1}: U_{1}\left(\mathbb{Z} C_{p^{n}}\right) \rightarrow \operatorname{Im}\left(\bar{\pi}_{1}\right)=\left\langle\mathcal{U}_{0}\right\rangle$, que foi definido no início do capítulo. Mais ainda, $\bar{\pi}_{1}(S)=\mathcal{U}_{0}$.

Verifiquemos então que $\left.\bar{\pi}_{1}\right|_{\langle S\rangle}:\langle S\rangle \rightarrow\left\langle\mathcal{U}_{0}\right\rangle$ é injetora, ou seja, $\operatorname{ker}\left(\left.\bar{\pi}_{1}\right|_{\langle S\rangle}\right)=\{1\}$.

Seja $\bar{\pi}_{1}\left(g^{i_{0}} \vartheta_{1}^{i_{1}} \vartheta_{2}^{i_{2}} \cdots \vartheta_{\kappa}^{i_{\kappa}}\right)=1$, com $0 \leq i_{0} \leq p^{n}-1$ e $i_{1}, \cdots, i_{\kappa} \in \mathbb{Z}$. Então $\theta^{i_{0}} h_{1}^{i_{1}} h_{2}^{i_{2}} \cdots h_{\kappa}^{i_{\kappa}}=1$ e, portanto, $1=\left(\theta^{i_{0}} h_{1}^{i_{1}} h_{2}^{i_{2}} \cdots h_{\kappa}^{i_{\kappa}}\right)^{p^{n}}=h_{1}^{i_{1} p^{n}} h_{2}^{i_{2} p^{n}} \cdots h_{\kappa}^{i_{\kappa} p^{n}}$. Como $h_{1}, h_{2}, \cdots, h_{\kappa}$ são multiplicativamente independentes, temos $i_{1}=i_{2}=\cdots=i_{\kappa}=0$. Logo $1=\bar{\pi}_{1}(x)=\bar{\pi}_{1}\left(g^{i_{0}}\right)=\theta^{i_{0}}$ e, portanto, $i_{0}=0$. Assim, $\operatorname{ker}\left(\left.\bar{\pi}_{1}\right|_{\langle S\rangle}\right)=\{1\}$, então $\langle S\rangle \cong\left\langle\mathcal{U}_{0}\right\rangle$. ¿

Com este resultado temos os seguintes isomorfismos:

$$
\langle S\rangle \cong \operatorname{Im}\left(\bar{\pi}_{1}\right) \cong \frac{U_{1}\left(\mathbb{Z} C_{p^{n}}\right)}{\operatorname{ker}\left(\bar{\pi}_{1}\right)}
$$

Vale ainda:

Teorema 4.4. $U_{1}\left(\mathbb{Z} C_{p^{n}}\right) \cong \operatorname{ker}\left(\bar{\pi}_{1}\right) \times \operatorname{Im}\left(\bar{\pi}_{1}\right)$.

\section{Prova:}

Seja $\iota: \operatorname{ker}\left(\bar{\pi}_{1}\right) \rightarrow U_{1}\left(\mathbb{Z} C_{p^{n}}\right)$ a inclusão. Temos a seguinte sequência exata curta:

$$
\{1\} \longrightarrow \operatorname{ker}\left(\bar{\pi}_{1}\right) \stackrel{\iota}{\longrightarrow} U_{1}\left(\mathbb{Z} C_{p^{n}}\right) \stackrel{\bar{\pi}_{1}}{\longrightarrow} \operatorname{Im}\left(\bar{\pi}_{1}\right) \longrightarrow\{1\}
$$

Se esta sequência cinde, então $U_{1}\left(\mathbb{Z} C_{p^{n}}\right) \simeq \operatorname{ker}\left(\bar{\pi}_{1}\right) \times \operatorname{Im}\left(\bar{\pi}_{1}\right)$ e assim teremos concluímos a nossa prova. Para isto mostraremos que existe um homomorfismo $\tau: \operatorname{Im}\left(\bar{\pi}_{1}\right) \rightarrow U_{1}\left(\mathbb{Z} C_{p^{n}}\right)$ tal que $\bar{\pi}_{1} \circ \tau=i d_{\operatorname{Im}\left(\bar{\pi}_{1}\right)}$. 
O Teorema das Unidades de Dirichlet nos diz que $U_{1}(\mathbb{Z}[\theta])=\langle\theta\rangle \times L$, onde $L$ é um grupo abeliano livre de posto finito. $\operatorname{Logo} \operatorname{Im}\left(\bar{\pi}_{1}\right)$ é um grupo abeliano finitamente gerado, digamos $\operatorname{Im}\left(\bar{\pi}_{1}\right)=T \times L_{1}$, onde $T$ é um grupo de torção (portanto, subgrupo de $\langle\theta\rangle$ ) e $L_{1}$ é um grupo abeliano livre. Agora, como $\langle\theta\rangle=\bar{\pi}_{1}(\langle g\rangle)$, temos $T=\langle\theta\rangle$ e, portanto, $\operatorname{Im}\left(\bar{\pi}_{1}\right)=\langle\theta\rangle \times L_{1}$.

Como $L_{1}$ é livre, então é projetivo. Logo existe $\tau_{1}: L_{1} \rightarrow U_{1}\left(\mathbb{Z} C_{p^{n}}\right)$ tal que $\bar{\pi}_{1} \circ \tau_{1}(u)=u$, para todo $u \in L_{1}$. Definimos $\tau: \operatorname{Im}\left(\bar{\pi}_{1}\right) \rightarrow U_{1}\left(\mathbb{Z} C_{p^{n}}\right)$ por $\tau\left(u \cdot \theta^{i}\right)=\tau_{1}(u) \cdot g^{i}$. Assim $\tau$ está bem definida e $\bar{\pi}_{1} \circ \tau=i d_{\operatorname{Im}\left(\bar{\pi}_{1}\right)}$.

Logo a sequência acima cinde, o que conclui a nossa prova. ¿

Como já sabemos, se $p$ é primo ímpar, então $U_{1}\left(\mathbb{Z} C_{p^{n}}\right)=C_{p^{n}} \times U_{*}\left(\mathbb{Z} C_{p^{n}}\right)$ e, se $p=2$, então $U_{1}\left(\mathbb{Z} C_{p^{n}}\right)=C_{p^{n}} \times U_{2}\left(\mathbb{Z} C_{p^{n}}\right)$, lembrando que $U_{*}\left(\mathbb{Z} C_{p^{n}}\right)$ é o conjunto das unidades simétricas normalizadas de $\mathbb{Z} C_{p^{n}}$ e $U_{2}\left(\mathbb{Z} C_{p^{n}}\right)=\left\{u \in U(\mathbb{Z} G): u \equiv 1 \bmod (\Delta G)^{2}\right\}$.

Ainda tem-se $U_{2}\left(\mathbb{Z} C_{p^{n}}\right) \subseteq U_{*}\left(\mathbb{Z} C_{p^{n}}\right)$. Então a unidade $w \in \operatorname{ker}\left(\bar{\pi}_{1}\right)$ pode ser decomposta da seguinte maneira: $w=g^{i} w^{\prime}$, para algum inteiro $i, 0 \leq i \leq p^{n}-1$ e $w^{\prime} \in U_{*}\left(\mathbb{Z} C_{p^{n}}\right)$. No caso $p=2$ podemos ter dois casos para $w^{\prime}$, portanto, devemos tomar o cuidado de $w^{\prime}$ pertencer a $U_{2}\left(\mathbb{Z} C_{p^{n}}\right)$. Se $(-1)^{p}\left(\mu_{t}\right)^{\frac{\phi\left(p^{n}\right)}{2 p}} \notin \operatorname{Im}\left(\bar{\pi}_{1}\right)$, então vamos identificar $\operatorname{Im}\left(\bar{\pi}_{1}\right)$ do seguinte modo:

$$
\operatorname{Im}\left(\bar{\pi}_{1}\right)=\langle\theta\rangle \times\left\langle\left\{h_{1}^{\prime}, h_{2}^{\prime}, h_{3}^{\prime} \cdots, h_{\kappa}^{\prime}\right\}\right\rangle \simeq\langle g\rangle \times\left\langle\left\{\vartheta_{1}^{\prime}, \vartheta_{2}^{\prime}, \vartheta_{3}^{\prime}, \cdots, \vartheta_{\kappa}^{\prime}\right\}\right\rangle
$$

onde $\vartheta_{i}=g^{n_{i}} \vartheta_{i}^{\prime}$, com $n_{i} \in \mathbb{Z}, \vartheta_{i}^{\prime} \in U_{*}\left(\mathbb{Z} C_{p^{n}}\right)$, no caso $p$ ímpar, e $\vartheta_{i}^{\prime} \in U_{2}\left(\mathbb{Z} C_{p^{n}}\right)$ no caso $p=2$. E ainda $h_{i}^{\prime}=\bar{\pi}_{1}\left(\vartheta_{i}^{\prime}\right)$.

Logo se $(-1)^{p}\left(\mu_{t}\right)^{\frac{\phi\left(p^{n}\right)}{2 p}} \notin \operatorname{Im}\left(\bar{\pi}_{1}\right)$, então $U_{1}\left(\mathbb{Z} C_{p^{n}}\right)=C_{p^{n}} \times k e r\left(\bar{\pi}_{1}\right) \times\left\langle\left\{\vartheta_{1}^{\prime}, \vartheta_{2}^{\prime}, \vartheta_{3}^{\prime}, \cdots, \vartheta_{\kappa}^{\prime}\right\}\right\rangle$. Utilizamos as unidades simétricas, pois elas são mais convenientes.

As unidades de $\mathbb{Z} C_{9}$ foram encontradas por Aleev e Panina no artigo [2], mas também o faremos no exemplo a seguir utilizando o nosso método.

Exemplo 4.1. Vamos encontrar $U_{1}\left(\mathbb{Z} C_{9}\right)$.

Mostraremos na última seção deste capítulo que $-\mu_{2} \notin \operatorname{Im}\left(\bar{\pi}_{1}\right)$.

$O$ posto de $U_{*}\left(\mathbb{Z} C_{3^{2}}\right)$ é $\frac{1}{2}\left(3^{2}-2(2+1)+1\right)=2, \kappa=\frac{\phi\left(3^{2}\right)}{2}-1=2, t=2, r=5 e$

$$
\vartheta_{i}=\left(1+g^{t}+g^{2 t}+\cdots+g^{(r-1) t}\right)\left(1+g^{t^{i}}+g^{2 t^{i}}+\cdots+g^{(t-1) t^{i}}\right)-\frac{(t r-1)}{p^{n}} \widehat{g},
$$


onde $\widehat{g}=\left(1+g+g^{2}+\cdots+g^{p^{n}-1}\right)$. Logo

$$
\begin{aligned}
S & =\left\{\vartheta_{1}=\left(1+g^{2}+g^{4}+g^{6}+g^{8}\right)\left(1+g^{2}\right)-\widehat{g}, \vartheta_{2}=\left(1+g^{2}+g^{4}+g^{6}+g^{8}\right)\left(1+g^{4}\right)-\widehat{g}\right\} \\
& =\left\{\vartheta_{1}=\left(g^{2}-g^{3}+g^{4}-g^{5}+g^{6}-g^{7}+g^{8}\right), \vartheta_{2}=\left(g^{4}-g^{5}+g^{6}-g^{7}+g^{8}\right)\right\} \\
& =\left\{\vartheta_{1}=g^{5}\left(-1+g-g^{2}+g^{3}+g^{6}-g^{7}+g^{8}\right), \vartheta_{2}=g^{6}\left(1-g+g^{2}+g^{7}-g^{8}\right)\right\} e
\end{aligned}
$$

$\vartheta_{1}^{\prime}=\left(-1+g-g^{2}+g^{3}+g^{6}-g^{7}+g^{8}\right) e \vartheta_{2}^{\prime}=\left(1-g+g^{2}+g^{7}-g^{8}\right)$ são unidades simétricas de $\mathbb{Z} C_{3^{2}}$. Neste caso temos $\operatorname{ker}\left(\bar{\pi}_{1}\right)=\{1\}$, pois o posto de $U_{*}\left(\mathbb{Z} C_{3^{2}}\right)$ é $\frac{1}{2}\left(3^{2}-2(2+1)+1\right)=2=\kappa$. Então $U\left(\mathbb{Z} C_{3^{2}}\right)= \pm U_{1}\left(\mathbb{Z} C_{3^{2}}\right)= \pm\langle S\rangle= \pm C_{3^{2}} \times\left\langle\left\{\vartheta_{1}^{\prime}, \vartheta_{2}^{\prime}\right\}\right\rangle$.

\subsection{Geradores Independentes para $\operatorname{ker}\left(\bar{\pi}_{1}\right)$}

Pelos Teoremas 4.3 e 4.4, se $(-1)^{p}\left(\mu_{t}\right)^{\frac{\phi\left(p^{n}\right)}{2 p}} \notin \operatorname{Im}\left(\bar{\pi}_{1}\right)$, então

$$
U_{1}\left(\mathbb{Z} C_{p^{n}}\right)=\operatorname{ker}\left(\bar{\pi}_{1}\right) \times\langle S\rangle,
$$

onde $S=\left\{g, \vartheta_{1}, \vartheta_{2}, \vartheta_{3}, \cdots, \vartheta_{\kappa}\right\}$ e $\vartheta_{i}$ é a unidade de Hoechsmann dada por

$\vartheta_{i}=\left(1+g^{t}+g^{2 t}+\cdots+g^{(r-1) t}\right)\left(1+g^{t^{i}}+g^{2 t^{i}}+\cdots+g^{(t-1) t^{i}}\right)-\frac{(t r-1)}{p^{n}}\left(1+g+g^{2}+\cdots+g^{p^{n}-1}\right)$.

Falta verificarmos quais são os elementos que geram $\operatorname{ker}\left(\bar{\pi}_{1}\right)$.

Primeiramente observe que as unidades $w \in \operatorname{ker}\left(\bar{\pi}_{1}\right)$ são simétricas.

Proposição 4.5. Se $w \in k e r\left(\bar{\pi}_{1}\right)$, então w é uma unidade simétrica.

\section{Prova:}

Seja $w \in \operatorname{ker}\left(\bar{\pi}_{1}\right)$ e seja $w=g^{i} \cdot w^{\prime}$, para algum inteiro $i, 0 \leq i \leq p^{n}-1$ e $w^{\prime} \in U_{*}\left(\mathbb{Z} C_{p^{n}}\right)$. Então $1=\bar{\pi}_{1}(w)=\theta^{i} \cdot \bar{\pi}_{1}\left(w^{\prime}\right)$ e, portanto, $\bar{\pi}_{1}\left(w^{\prime}\right)=\theta^{-i}$. Como $w^{\prime}$ é simétrica, $\bar{\pi}_{1}\left(w^{\prime}\right)=\theta^{-i}$ também deve ser simétrica.

Porém, no caso em que $p$ é primo ímpar, o único inteiro $i$ que torna $\theta^{-i}$ simétrico é $i=0$. Logo $w=g^{0} \cdot w^{\prime}=w^{\prime}$ e, portanto, $w$ é uma unidade simétrica. 
No caso em que $p=2$, temos que o elemento $\theta^{-i}$ é simétrico quando $i=0$ e $i=2^{n-1}$. Nestes casos, temos $w=g^{0} \cdot w^{\prime}=w^{\prime}$ ou $w=g^{2^{n-1}} \cdot w^{\prime}$. Como $g^{2^{n-1}}$ e $w^{\prime}$ são simétricos, então $w$ é uma unidade simétrica. is

Até o final desta seção, vamos provar os resultados para o caso $p$ ímpar e, portanto, $U_{*}\left(\mathbb{Z} C_{p^{n}}\right)$ será a componente livre de torção de $U_{1}\left(\mathbb{Z} C_{p^{n}}\right)$. Para o caso $p=2$, a prova é a mesma substituindo $U_{*}\left(\mathbb{Z} C_{p^{n}}\right)$ por $U_{2}\left(\mathbb{Z} C_{p^{n}}\right)$.

Como citamos na seção anterior,

$$
\operatorname{Im}\left(\bar{\pi}_{1}\right)=\langle\theta\rangle \times\left\langle\left\{h_{1}^{\prime}, h_{2}^{\prime}, h_{3}^{\prime} \cdots, h_{\kappa}^{\prime}\right\}\right\rangle \simeq\langle g\rangle \times\left\langle\left\{\vartheta_{1}^{\prime}, \vartheta_{2}^{\prime}, \vartheta_{3}^{\prime}, \cdots, \vartheta_{\kappa}^{\prime}\right\}\right\rangle
$$

Com estas notações provamos o seguinte resultado:

Proposição 4.6. Sejam $p$ um primo impar tal que $\phi\left(p^{n}\right) \leq 66$ e $\bar{\pi}_{1}: U_{1}\left(\mathbb{Z} C_{p^{n}}\right) \rightarrow U_{1}(\mathbb{Z}[\theta]) o$ homomorfismo de grupos definido anteriormente. Se $(-1)^{p}\left(\mu_{t}\right)^{\frac{\phi\left(p^{n}\right)}{2 p}} \notin \operatorname{Im}\left(\bar{\pi}_{1}\right)$, então o posto de $U_{*}\left(\mathbb{Z} C_{p^{n-1}}\right)$ é igual ao posto de $\operatorname{ker}\left(\bar{\pi}_{1}\right)$.

\section{Prova:}

Pelo Teorema 1.4, o posto de $U_{*}\left(\mathbb{Z} C_{p^{n}}\right)$ e de $\left.U_{*}\left(\mathbb{Z} C_{p^{n-1}}\right)\right)$ são $\left.\frac{1}{2}\left(p^{n}-2(n+1)+1\right)\right)=\frac{1}{2}\left(p^{n}-2 n-1\right)$ e $\left.\frac{1}{2}\left(p^{n-1}-2(n-1+1)+1\right)\right)=\frac{1}{2}\left(p^{n-1}-2 n+1\right)$, respectivamente.

Por hipótese, $(-1)^{p}\left(\mu_{t}\right)^{\frac{\phi\left(p^{n}\right)}{2 p}} \notin \operatorname{Im}\left(\bar{\pi}_{1}\right)$, então $\operatorname{Im}\left(\bar{\pi}_{1}\right)=\left\langle\mathcal{U}_{0}\right\rangle=\left\langle\left\{\theta, h_{1}, h_{2}, \cdots, h_{\kappa}\right\}\right\rangle$ e a componente livre de torção de $\operatorname{Im}\left(\bar{\pi}_{1}\right)$ é $H=\left\langle\left\{h_{1}^{\prime}, h_{2}^{\prime}, \cdots, h_{\kappa}^{\prime}\right\}\right\rangle$.

Temos ainda a seguinte sequência exata curta:

$$
\{1\} \longrightarrow \operatorname{ker}\left(\bar{\pi}_{1}\right) \stackrel{\iota}{\longrightarrow} U_{*}\left(\mathbb{Z} C_{p^{n}}\right) \stackrel{\bar{\pi}_{1}}{\longrightarrow} H \longrightarrow\{1\}
$$

onde $\iota: \operatorname{ker}\left(\bar{\pi}_{1}\right) \rightarrow U_{1}\left(\mathbb{Z} C_{p^{n}}\right)$ é a inclusão.

Analogamente, como mostramos no Teorema 4.4, prova-se que esta sequência exata cinde e, portanto, $U_{*}\left(\mathbb{Z} C_{p^{n}}\right) \simeq \operatorname{ker}\left(\bar{\pi}_{1}\right) \times H$. Sabemos que, para um grupo livre $G$ de posto $m$ vale o isomorfismo $G \simeq \underbrace{\mathbb{Z} \times \mathbb{Z} \times \cdots \times \mathbb{Z}}_{m \text { vezes }}, \operatorname{logo} U_{*}\left(\mathbb{Z} C_{p^{n}}\right) \simeq \underbrace{\mathbb{Z} \times \mathbb{Z} \times \cdots \times \mathbb{Z}}_{\frac{1}{2}\left(p^{n}-2 n-1\right) \text { vezes }}$ e $H \simeq \underbrace{\mathbb{Z} \times \mathbb{Z} \times \cdots \times \mathbb{Z}}_{\kappa \text { vezes }}$.

Assim,

$$
\underbrace{\mathbb{Z} \times \mathbb{Z} \times \cdots \times \mathbb{Z}}_{\frac{1}{2}\left(p^{n}-2 n-1\right) \text { vezes }} \simeq U_{*}\left(\mathbb{Z} C_{p^{n}}\right) \simeq \operatorname{ker}\left(\bar{\pi}_{1}\right) \times H \simeq \operatorname{ker}\left(\bar{\pi}_{1}\right) \times \underbrace{\mathbb{Z} \times \mathbb{Z} \times \cdots \times \mathbb{Z}}_{\kappa \text { vezes }} .
$$

Como o posto de um grupo livre é único, então o posto de $k e r\left(\bar{\pi}_{1}\right)$ é $\frac{1}{2}\left(p^{n}-2 n-1\right)-\kappa$. 
Agora,

$$
\begin{aligned}
\frac{1}{2}\left(p^{n}-2 n-1\right)-\kappa & =\frac{\left(p^{n}-2 n-1\right)}{2}-\left(\frac{p^{n-1}(p-1)}{2}-1\right) \\
& =\frac{1}{2}\left(p^{n-1}-2 n+1\right) .
\end{aligned}
$$

Como $\frac{1}{2}\left(p^{n-1}-2 n+1\right)$ é o posto de $U_{*}\left(\mathbb{Z} C_{p^{n-1}}\right)$, então concluimos a nossa prova. «

Sejam $f_{1}: \mathbb{Z} C_{p^{n-1}} \rightarrow \mathbb{Z}_{p} C_{p^{n-1}}$ o homomorfismo natural de anéis e $\bar{f}_{1}:=\left.f_{1}\right|_{U_{*}\left(\mathbb{Z} C_{p^{n-1}}\right)}$. Pelo teorema do homomorfismo para grupos, temos $\frac{U_{*}\left(\mathbb{Z} C_{p^{n-1}}\right)}{\operatorname{ker}\left(\bar{f}_{1}\right)} \simeq \operatorname{Im}\left(\bar{f}_{1}\right)$ e como $\operatorname{Im}\left(\bar{f}_{1}\right)$ é finito, então o posto de $\operatorname{ker}\left(\bar{f}_{1}\right)$ é igual ao de $U_{*}\left(\mathbb{Z} C_{p^{n-1}}\right)$. Com esta informação e com a Proposição 4.6 resulta que o posto de $\operatorname{ker}\left(\bar{\pi}_{1}\right)$ e de $\operatorname{ker}\left(\bar{f}_{1}\right)$ são iguais.

Sabemos que $\operatorname{ker}\left(\pi_{1}\right)$ é um ideal de $\mathbb{Z} C_{p^{n}}$ gerado por $\sum_{i=0}^{p-1} g^{i p^{n-1}}$ e denotaremos esta soma por $\left(\widehat{g^{p^{n-1}}}\right)$. Deste modo

$$
\operatorname{ker}\left(\pi_{1}\right)=\left\{a_{0}\left(\widehat{g^{p^{n-1}}}\right)+a_{1} g\left(\widehat{g^{p^{n-1}}}\right)+\cdots+a_{p^{n-1}-1} g^{p^{n-1}-1}\left(\widehat{g^{p^{n-1}}}\right) \mid a_{i} \in \mathbb{Z}\right\}
$$

e, portanto,

$$
\operatorname{ker}\left(\bar{\pi}_{1}\right)=\left\{1+a_{0}\left(\widehat{g^{p^{n-1}}}\right)+a_{1} g\left(\widehat{g^{p^{n-1}}}\right)+\cdots+a_{p^{n-1}-1} g^{p^{n-1}-1}\left(\widehat{g^{p^{n-1}}}\right) \in U\left(\mathbb{Z} C_{p^{n}}\right) \mid a_{i} \in \mathbb{Z}\right\}
$$

Vamos analisar melhor os elementos de $\operatorname{ker}\left(\bar{\pi}_{1}\right)$. O que sabemos é que se $w \in \operatorname{ker}\left(\bar{\pi}_{1}\right)$, então $w \in U_{*}\left(\mathbb{Z} C_{p^{n}}\right)$ e é da forma $w=1+a_{0}\left(\widehat{g^{p^{n-1}}}\right)+a_{1} g\left(\widehat{g^{p^{n-1}}}\right)+\cdots+a_{p^{n-1}-1} g^{p^{n-1}-1}\left(\widehat{g^{p^{n-1}}}\right)$. Mas não sabemos ainda quais inteiros $a_{0}, a_{1}, \cdots, a_{p^{n-1}-1}$ tornam $w$ um dos geradores de $\operatorname{ker}\left(\bar{\pi}_{1}\right)$. Então o que faremos a seguir é tentar encontrar estes inteiros.

Aplicando $\pi_{2}: \mathbb{Z} C_{p^{n}} \longrightarrow \mathbb{Z} C_{p^{n-1}}$ em $w$, temos $\pi_{2}(w) \in U_{*}\left(\mathbb{Z} C_{p^{n-1}}\right)$ e

$$
\pi_{2}(w)=1+p\left(a_{0}+a_{1} h+a_{2} h^{2}+\cdots+a_{p^{n-1}-2} h^{p^{n-1}-2}+a_{p^{n-1}-1} h^{p^{n-1}-1}\right),
$$

pois $1+h^{p^{n-1}}+h^{2 p^{n-1}}+\cdots+h^{(p-1) p^{n-1}}=p$ já que $h^{p^{n-1}}=1$.

Então devemos manipular os elementos de $U_{*}\left(\mathbb{Z} C_{p^{n-1}}\right)$ tais que tenham a forma $1+p v$, com $v \in \mathbb{Z} C_{p^{n-1}}$, para encontrar os inteiros $a_{i}$ 's.

Mas facilmente verifica-se que $\operatorname{ker}\left(\bar{f}_{1}\right)=\left\{1+p v \in U_{*}\left(\mathbb{Z} C_{p^{n-1}}\right) \mid v \in \mathbb{Z} C_{p^{n-1}}\right\}$. Logo, se encontrarmos os geradores de $\operatorname{ker}\left(\bar{f}_{1}\right)$, então encontraremos os geradores de $\operatorname{ker}\left(\bar{\pi}_{1}\right)$ do seguinte modo: 
Como o posto de $\operatorname{ker}\left(\bar{f}_{1}\right)$ e de $\operatorname{ker}\left(\bar{\pi}_{1}\right)$ são iguais então, para cada gerador $u_{i}=1+p v_{i}$ de $\operatorname{ker}\left(\bar{f}_{1}\right)$, fazemos a seguinte igualdade:

$$
u_{i}=1+p v_{i}=\pi_{2}(w)=1+p\left(a_{0}+a_{1} h+a_{2} h^{2}+\cdots+a_{p^{n-1}-2} h^{p^{n-1}-2}+a_{p^{n-1}-1} h^{p^{n-1}-1}\right) .
$$

Desta igualdade encontramos os coeficientes $a_{i}$ 's de $w=1+a_{0}\left(\widehat{g^{p^{n-1}}}\right)+\cdots+a_{p^{n-1}-1} g^{p^{n-1}-1}\left(\widehat{g^{p^{n-1}}}\right)$. A proposição a seguir mostra que $w$ formado deste modo é único para cada $u_{i}$ e o Teorema 1.27 garante que $w$ é uma unidade de $\mathbb{Z} C_{p^{n}}$. Portanto, estes elementos $w$ formam uma base para $\operatorname{ker}\left(\bar{\pi}_{1}\right)$.

Proposição 4.7. Seja $\pi_{2}: U_{1}\left(\mathbb{Z} C_{p^{n}}\right) \rightarrow U_{1}\left(\mathbb{Z} C_{p^{n-1}}\right)$ o homomorfismo que definimos inicialmente. Então $\left.\pi_{2}\right|_{\text {ker }\left(\bar{\pi}_{1}\right)}$ é injetora.

\section{Prova:}

Seja $w \in \operatorname{ker}\left(\bar{\pi}_{1}\right) \cap \operatorname{ker}\left(\pi_{2}\right)$. Então $w=1+a_{0}\left(\widehat{g^{p^{n-1}}}\right)+a_{1} g\left(\widehat{g^{p^{n-1}}}\right)+\cdots+a_{p^{n-1}-1} g^{p^{n-1}-1}\left(\widehat{g^{p^{n-1}}}\right)$ e $\pi_{2}(w)=1+p\left(a_{0}+a_{1} h+a_{2} h^{2}+\cdots+a_{p^{n-1}-2} h^{p^{n-1}-2}+a_{p^{n-1}-1} h^{p^{n-1}-1}\right)=1$. Deste modo $a_{0}=a_{1}=\cdots=a_{p^{n-1}-1}=0$, portanto, $w=1$. Logo $\left.\pi_{2}\right|_{k e r\left(\bar{\pi}_{1}\right)}$ é injetora. \&

Como o procedimento acima, o problema de encontrarmos uma base de $\operatorname{ker}\left(\bar{\pi}_{1}\right)$ se restringe agora a encontrarmos uma base de $\operatorname{ker}\left(\bar{f}_{1}\right)$.

Pelo teorema do homomorfismo para grupos, temos $\left|\operatorname{Im}\left(\bar{f}_{1}\right)\right|=\left|U_{*}\left(\mathbb{Z} C_{p^{n-1}}\right): \operatorname{ker}\left(\bar{f}_{1}\right)\right|$. Por outro lado, se $N$ é um subgrupo de $\operatorname{ker}\left(\bar{f}_{1}\right)$ tal que $\left|\operatorname{Im}\left(\bar{f}_{1}\right)\right|=\left|U_{*}\left(\mathbb{Z} C_{p^{n-1}}\right): N\right|$, então $N=\operatorname{ker}\left(\bar{f}_{1}\right)$. O que faremos é encontrar este $N$.

Suponha que se conheça uma base para $U_{*}\left(\mathbb{Z} C_{p^{n-1}}\right)$, digamos $B=\left\{u_{1}, u_{2}, \cdots, u_{r}\right\}$. Já conhecemos uma base para $U_{1}\left(\mathbb{Z} C_{p}\right)$ pelo artigo [15]. Então $\operatorname{Im}\left(\bar{f}_{1}\right)=\left\langle\bar{f}_{1}(B)\right\rangle=$ $\left\langle\left\{\bar{f}_{1}\left(u_{1}\right), \bar{f}_{1}\left(u_{2}\right), \cdots, \bar{f}_{1}\left(u_{r}\right)\right\}\right\rangle$. Como $\left\{\bar{f}_{1}\left(u_{1}\right), \bar{f}_{1}\left(u_{2}\right), \cdots, \bar{f}_{1}\left(u_{r}\right)\right\}$ não precisa ser multiplicativamente independente, é possível encontrar a partir de $B$ um conjunto multiplicativamente independente $B^{\prime}$ que gere $\left\langle\bar{f}_{1}(B)\right\rangle$. Deste conjunto multiplicativamente independente encontramos a ordem de $\operatorname{Im}\left(\bar{f}_{1}\right)$. Ainda por este procedimento de encontrar $B^{\prime}$, encontramos os elementos que compõem a base $\operatorname{ker}\left(\bar{f}_{1}\right)$. Na proposição a seguir encontramos alguns elementos do núcleo de $\bar{f}_{1}$.

Proposição 4.8. Seja $w=1+a_{0}\left(\widehat{g^{p^{m-1}}}\right)+a_{1} g\left(\widehat{g^{p^{m-1}}}\right)+\cdots+a_{p^{m-1}-1} g^{p^{m-1}-1}\left(\widehat{g^{p^{m-1}}}\right) \in U_{*}\left(\mathbb{Z} C_{p^{m}}\right)$ e sejam $f_{1}: \mathbb{Z} C_{p^{m}} \rightarrow \mathbb{Z}_{p} C_{p^{m}}$ o homomorfismo usual e $\bar{f}_{1}=\left.f_{1}\right|_{U_{*}\left(\mathbb{Z} C_{p^{m}}\right)}$. Então $w^{p} \in \operatorname{ker}\left(\bar{f}_{1}\right)$. 


\section{Prova:}

Primeiramente observe que

$$
\left(\widehat{g^{p^{m-1}}}\right)^{p}=\left(\overline{1}+g^{p \cdot p^{m-1}}+g^{p \cdot 2 \cdot p^{m-1}}+\cdots+g^{p \cdot(p-1) \cdot p^{m-1}}\right)=\bar{p}=\overline{0} \in \mathbb{Z}_{p} C_{p^{m}}
$$

e como $\bar{f}_{1}\left(w^{p}\right) \in \mathbb{Z}_{p} C_{p^{m}}$, temos:

$$
\begin{aligned}
\bar{f}_{1}\left(w^{p}\right) & =\left[\overline{1}+\bar{a}_{0}\left(\widehat{g^{p^{m-1}}}\right)+\bar{a}_{1} g\left(\widehat{g^{p^{m-1}}}\right)+\cdots+\bar{a}_{p^{m-1}-1} g^{p^{m-1}-1}\left(\widehat{g^{p^{m-1}}}\right)\right]^{p} \\
& =\overline{1}+\left(\bar{a}_{0}\right)^{p}\left(\widehat{g^{p^{m-1}}}\right)^{p}+\left(\bar{a}_{1}\right)^{p} g^{p}\left(\widehat{g^{p^{m-1}}}\right)^{p}+\cdots+\left(\bar{a}_{p^{m-1}-1}\right)^{p} g^{\left(p^{m-1}-1\right) \cdot p}\left(\widehat{g^{p^{m-1}}}\right)^{p} \\
& =\overline{1}+\bar{p} \cdot\left[\left(\bar{a}_{0}\right)^{p}+\left(\bar{a}_{1}\right)^{p} g^{p}+\cdots+\left(\bar{a}_{p^{m-1}-1}\right)^{p} g^{\left(p^{m-1}-1\right) \cdot p}\right] \\
& =\overline{1} .
\end{aligned}
$$

$\operatorname{Logo} w^{p} \in \operatorname{ker}\left(\bar{f}_{1}\right) . \boldsymbol{z}$

Antes de encontrarmos os geradores de $\operatorname{ker}\left(\bar{f}_{1}\right)$, façamos uma observação: sabe-se que a ordem de $U_{1}\left(\mathbb{Z}_{p} C_{p^{n}}\right)$ é $p^{p^{n}-1}, \operatorname{logo} U_{1}\left(\mathbb{Z}_{p} C_{p^{n}}\right)$ é um $p$-grupo. Usaremos este fato para calcular a ordem de $\operatorname{Im}\left(\bar{f}_{1}\right)$ nos exemplos a seguir.

Não conseguimos um método geral para encontrar uma base para $\operatorname{ker}\left(\bar{f}_{1}\right)$, apesar da ideia para encontrá-las ser parecida em todos os casos. Exibiremos agora as bases para $k e r\left(\bar{\pi}_{1}\right)$ nos casos que estamos interessados. Mais detalhes dos cálculos a seguir podem ser encontradas no apêndice.

Exemplo 4.2. Vamos encontrar $\operatorname{ker}\left(\bar{\pi}_{1}\right)$ no caso $\mathbb{Z} C_{3^{3}}$.

Sejam $C_{3^{3}}=\langle g\rangle, C_{9}=\langle h\rangle$ e $x=h-1$. Vamos analisar o diagrama abaixo:

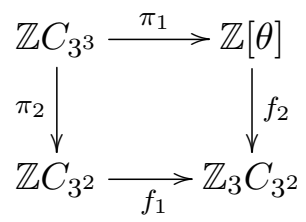

Para encontrar $\operatorname{ker}\left(\bar{\pi}_{1}\right)$, procuraremos um subgrupo $N$ de $\operatorname{ker}\left(\bar{f}_{1}\right)$ tal que

$$
\left|U_{*}\left(\mathbb{Z} C_{9}\right): N\right|=\left|\operatorname{Im}\left(\bar{f}_{1}\right)\right|
$$


Primeiramente iremos encontrar a ordem de $\operatorname{Im}\left(\bar{f}_{1}\right)$. Sabemos que

$$
U_{*}\left(\mathbb{Z} C_{9}\right)=\left\langle\left\{u_{1}=-1+h-h^{2}+h^{3}+h^{6}-h^{7}+h^{8}, u_{2}=1-h+h^{2}+h^{7}-h^{8}\right\}\right\rangle .
$$

Então $\operatorname{Im}\left(\bar{f}_{1}\right)=\left\langle\bar{f}_{1}\left(u_{1}\right), \bar{f}_{1}\left(u_{2}\right)\right\rangle$. Seja $x=h-1$ e então $h=x+1$ e:

$$
\begin{aligned}
& \bar{f}_{1}\left(u_{1}\right)=-\overline{1}+h-\overline{1} h^{2}+h^{3}+h^{6}-\overline{1} h^{7}+h^{8}=\overline{1}+\overline{2} x^{4}+\overline{2} x^{5}+x^{6}+x^{7}+x^{8}, \\
& \bar{f}_{1}\left(u_{2}\right)=\overline{1}-\overline{1} h+h^{2}+h^{7}-\overline{1} h^{8}=\overline{1}+x^{4}+x^{5}+\overline{2} x^{7}+\overline{2} x^{8} .
\end{aligned}
$$

Vamos encontrar um conjunto multiplicativamente independente que gera $\operatorname{Im}\left(\bar{f}_{1}\right)$ :

$$
\begin{aligned}
\bar{f}_{1}\left(u_{1}\right) & =\overline{1}+\overline{2} x^{4}+\overline{2} x^{5}+x^{6}+x^{7}+x^{8} \\
\bar{f}_{1}\left(u_{1}\right) \cdot \bar{f}_{1}\left(u_{2}\right) & =\overline{1}+x^{6}+\overline{2} x^{8} .
\end{aligned}
$$

Logo $\bar{f}_{1}\left(u_{1}\right)$ e $\bar{f}_{1}\left(u_{1}\right) \cdot \bar{f}_{1}\left(u_{2}\right)$ são independentes e $\operatorname{Im}\left(\bar{f}_{1}\right)=\left\langle\bar{f}_{1}\left(u_{1}\right)\right\rangle \times\left\langle\bar{f}_{1}\left(u_{1}\right) \cdot \bar{f}_{1}\left(u_{2}\right)\right\rangle$. Como a ordem de $\bar{f}_{1}\left(u_{1}\right)$ e de $\bar{f}_{1}\left(u_{1}\right) \cdot \bar{f}_{1}\left(u_{2}\right)$ é 3, então a ordem de $\operatorname{Im}\left(\bar{f}_{1}\right)$ é 9 .

Seja $N=\left\langle u_{1}^{3}, u_{2}^{3}\right\rangle$. Temos:

$$
\begin{aligned}
& \bar{f}_{1}\left(u_{1}^{3}\right)=\left(\overline{1}+\overline{2} x^{4}+\overline{2} x^{5}+x^{6}+x^{7}+x^{8}\right)^{3}=\overline{1}+\overline{2}^{3} x^{4 \cdot 3}+\overline{2}^{3} x^{5 \cdot 3}+x^{6 \cdot 3}+x^{7 \cdot 3}+x^{8 \cdot 3}=\overline{1} e \\
& \bar{f}_{1}\left(u_{2}^{3}\right)=\left(\overline{1}+x^{4}+x^{5}+\overline{2} x^{7}+\overline{2} x^{8}\right)^{3}=\overline{1}+x^{4 \cdot 3}+x^{5 \cdot 3}+\overline{2}^{3} x^{7 \cdot 3}+\overline{2}^{3} x^{8 \cdot 3}=\overline{1},
\end{aligned}
$$

pois $x^{j}=\overline{0}$ para $j \geq 9$. Logo $N \subseteq \operatorname{ker}\left(\bar{f}_{1}\right)$ e como $U_{*}\left(\mathbb{Z} C_{9}\right)=\left\langle u_{1}, u_{2}\right\rangle$, concluímos a igualdade $\left|U_{*}\left(\mathbb{Z} C_{9}\right): N\right|=3^{2}=\left|\operatorname{Im}\left(\bar{f}_{1}\right)\right|$. Portanto, $N=\operatorname{ker}\left(\bar{f}_{1}\right)$.

Agora se

$w=1+a_{0} \widehat{\left(g^{9}\right)}+a_{1} g \widehat{\left(g^{9}\right)}+a_{2} g^{2} \widehat{\left(g^{9}\right)}+a_{3} g^{3} \widehat{\left(g^{9}\right)}+a_{4} g^{4} \widehat{\left(g^{9}\right)}+a_{5} g^{5} \widehat{\left(g^{9}\right)}+a_{6} g^{6} \widehat{\left(g^{9}\right)}+a_{7} g^{7} \widehat{\left(g^{9}\right)}+a_{8} g^{8} \widehat{\left(g^{9}\right)} \in \operatorname{ker}\left(\bar{\pi}_{1}\right)$,

então $\pi_{1}(w)=1+3 \cdot\left(a_{0}+a_{1} h+a_{2} h^{2}+\cdots+a_{8} h^{8}\right)$. Igualamos $\pi_{2}(w)=u_{i}^{3}$ e encontramos uma base para $\operatorname{ker}\left(\bar{\pi}_{1}\right)$, a saber,

$$
\begin{aligned}
u_{1}^{3} & =1+3\left(-12+11 h-9 h^{2}+6 h^{3}-2 h^{4}-2 h^{5}+6 h^{6}-9 h^{7}+11 h^{8}\right)=\pi_{2}(w) \\
& =1+3\left(a_{0}+a_{1} h+a_{2} h^{2}+a_{3} h^{3}+\cdots+a_{7} h^{7}+a_{8} h^{8}\right) .
\end{aligned}
$$

Logo encontramos $a_{0}=-12, a_{1}=a_{8}=11, a_{2}=a_{7}=-9, a_{3}=a_{6}=6$ e $a_{4}=a_{5}=-2$ e deste 
modo

$w=1-12 \widehat{\left(g^{9}\right)}+11 g \widehat{\left(g^{9}\right)}-9 g^{2} \widehat{\left(g^{9}\right)}+6 g^{3} \widehat{\left(g^{9}\right)}-2 g^{4} \widehat{\left(g^{9}\right)}-2 g^{5} \widehat{\left(g^{9}\right)}+6 g^{6} \widehat{\left(g^{9}\right)}-9 g^{7} \widehat{\left(g^{9}\right)}+11 g^{8} \widehat{\left(g^{9}\right)} \in k e r\left(\bar{\pi}_{1}\right)$.

Ainda encontramos o outro gerador de $\operatorname{ker}\left(\bar{\pi}_{1}\right)$, fazendo

$$
\begin{aligned}
u_{2}^{3} & =1+3\left(6-6 h+5 h^{2}-3 h^{3}+h^{4}+h^{5}-3 h^{6}+5 h^{7}-6 h^{8}\right)=\pi_{2}(w) \\
& =1+3\left(a_{0}+a_{1} h+a_{2} h^{2}+a_{3} h^{3}+\cdots+a_{7} h^{7}+a_{8} h^{8}\right)
\end{aligned}
$$

obtemos $a_{0}=6, a_{1}=a_{8}=-6, a_{2}=a_{7}=5, a_{3}=a_{6}=-3$ e $a_{4}=a_{5}=1$, daí

$$
1+6 \widehat{\left(g^{9}\right)}-6 g \widehat{\left(g^{9}\right)}+5 g^{2} \widehat{\left(g^{9}\right)}-3 g^{3} \widehat{\left(g^{9}\right)}+1 g^{4} \widehat{\left(g^{9}\right)}+1 g^{5} \widehat{\left(g^{9}\right)}-3 g^{6} \widehat{\left(g^{9}\right)}+5 g^{7} \widehat{\left(g^{9}\right)}-6 g^{8} \widehat{\left(g^{9}\right)}
$$

é o outro gerador que procuramos. Logo

$$
\begin{aligned}
& \quad \operatorname{ker}\left(\bar{\pi}_{1}\right)=\left\langle\left\{v_{1}, v_{2}\right\}\right\rangle= \\
& =\left\langle\left\{ v_{1}=1-12 \widehat{\left(g^{9}\right)}+11 g \widehat{\left(g^{9}\right)}-9 g^{2} \widehat{\left(g^{9}\right)}+6 g^{3} \widehat{\left(g^{9}\right)}-2 g^{4} \widehat{\left(g^{9}\right)}-2 g^{5} \widehat{\left(g^{9}\right)}+6 g^{6} \widehat{\left(g^{9}\right)}-9 g^{7} \widehat{\left(g^{9}\right)}+11 g^{8} \widehat{\left(g^{9}\right)},\right.\right. \\
& \left.\left.v_{2}=1+6 \widehat{\left(g^{9}\right)}-6 g \widehat{\left(g^{9}\right)}+5 g^{2} \widehat{\left(g^{9}\right)}-3 g^{3} \widehat{\left(g^{9}\right)}+1 g^{4} \widehat{\left(g^{9}\right)}+1 g^{5} \widehat{\left(g^{9}\right)}-3 g^{6} \widehat{\left(g^{9}\right)}+5 g^{7} \widehat{\left(g^{9}\right)}-6 g^{8} \widehat{\left(g^{9}\right)}\right\}\right\rangle
\end{aligned}
$$

Exemplo 4.3. Caso $\mathbb{Z} C_{3^{4}}$.

$\operatorname{Sejam~} C_{3^{4}}=\langle g\rangle, C_{3^{3}}=\langle h\rangle$ e $x=h-1$.

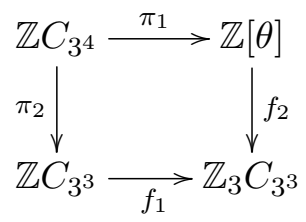

Vamos encontrar a ordem de $\operatorname{Im}\left(\bar{f}_{1}\right)$. Temos $U_{*}\left(\mathbb{Z} C_{3^{3}}\right)=\left\langle u_{1}^{\prime}, u_{2}^{\prime}, \cdots, u_{8}^{\prime}\right\rangle \times\left\langle u_{9}, u_{10}\right\rangle$, onde $u_{1}, u_{2}, \cdots, u_{8}$ são as unidades de Hoechsmann e $u_{9}, u_{10}$ são as unidades que encontramos no exemplo anterior. $u_{i}^{\prime}$ é a unidade simétrica que obtemos a partir de $u_{i}$.

$$
\text { Temos } u_{i}=\left(\sum_{j=0}^{13} h^{2 \cdot j}\right) \cdot\left(1+h^{2^{i}}\right)-\left(\sum_{j=0}^{26} h^{j}\right) \text { e } u_{i}^{\prime}=h^{m} \cdot\left(\sum_{j=0}^{13} h^{2 \cdot j}\right) \cdot\left(1+h^{2^{i}}\right)-\left(\sum_{j=0}^{26} h^{j}\right),
$$

onde $m$ é o inteiro que torna a unidade $u_{i}^{\prime}$ simétrica. 
Aplicando $\bar{f}_{1}$ e substituindo $h=x+1$ em cada uma das unidades acima, encontramos:

$$
\begin{aligned}
\bar{f}_{1}\left(u_{1}^{\prime}\right)= & 1+2 x^{4}+2 x^{5}+x^{6}+x^{7}+x^{8}+2 x^{9}+x^{10}+x^{11}+x^{12}+2\left(\sum_{i=15}^{26} x^{i}\right) \\
\bar{f}_{1}\left(u_{2}^{\prime}\right)= & 1+x^{4}+x^{5}+2 x^{7}+2 x^{8}+2 x^{10}+2 x^{11}+2 x^{12}+2 x^{15}+\left(\sum_{i=16}^{26} x^{i}\right) \\
\bar{f}_{1}\left(u_{3}^{\prime}\right)= & 1+2\left(x^{10}+x^{11}+x^{12}+x^{13}+x^{14}+x^{15}+x^{16}+x^{17}\right)+\left(\sum_{i=18}^{26} x^{i}\right) \\
\bar{f}_{1}\left(u_{4}^{\prime}\right)= & 1+2 x^{4}+2 x^{5}+x^{6}+x^{7}+x^{8}+2 x^{9}+x^{12}+2\left(\sum_{i=13}^{13} x^{i}\right)+x^{18}+2 x^{21}+\left(\sum_{i=22}^{26} x^{i}\right) \\
\bar{f}_{1}\left(u_{5}^{\prime}\right)= & 1+x^{4}+x^{5}+2 x^{7}+2 x^{8}+\left(\sum_{i=0}^{2} x^{10+6 i}+x^{11+6 i}+2\left(x^{13+6 i}+x^{14+6 i}\right)\right) \\
\bar{f}_{1}\left(u_{6}^{\prime}\right)= & 1+x^{10}+x^{11}+x^{12}+x^{13}+x^{14}+x^{15}+x^{16}+x^{17}+2\left(\sum_{i=19}^{26} x^{i}\right) \\
\bar{f}_{1}\left(u_{7}^{\prime}\right)= & 1+2 x^{4}+2 x^{5}+x^{6}+x^{7}+x^{8}+ \\
& +\left(\sum_{i=0}^{2} 2\left(x^{9+6 \cdot i}+x^{10+6 \cdot i}+x^{11+6 \cdot i}\right)+x^{12+6 \cdot i}+x^{13+6 \cdot i}+x^{14+6 \cdot i}\right) \\
\bar{f}_{1}\left(u_{10}\right)= & 1+x^{22}+x^{23}+2 x^{24}+2 x^{25} \\
\bar{f}_{1}\left(u_{8}^{\prime}\right)= & 1+x^{4}+x^{5}+2 x^{7}+2 x^{8}+\left(\sum_{i=12}^{17} x^{i}\right)+2\left(\sum_{i=21}^{26} x^{i}\right) \\
= & 1+2 x^{22}+2 x^{23}+2 x^{24}+x^{25}+2 x^{26} \\
= &
\end{aligned}
$$

Seja $H=\left\{\bar{f}_{1}\left(u_{1}^{\prime}\right), \bar{f}_{1}\left(u_{2}^{\prime}\right), \bar{f}_{1}\left(u_{3}^{\prime}\right), \cdots, \bar{f}_{1}\left(u_{10}\right)\right\}$. Em seguida reduzimos $H$ até encontrarmos um conjunto multiplicativamente independente $\left\{e_{1}, e_{2}, \cdots, e_{8}\right\}$ que gera o mesmo subgrupo que $\langle H\rangle=\operatorname{Im}\left(\bar{f}_{1}\right)$. Obtemos assim:

$$
\begin{aligned}
& e_{1}=\bar{f}_{1}\left(u_{1}^{\prime}\right)=1+2 x^{4}+2 x^{5}+x^{6}+x^{7}+x^{8}+2 x^{9}+x^{10}+x^{11}+x^{12}+2\left(\sum_{i=15}^{26} x^{i}\right) \\
& e_{2}=\bar{f}_{1}\left(u_{2}^{\prime} u_{1}^{\prime}\right)=1+x^{6}+2 x^{8}+x^{12}+2 x^{14}+x^{18}+2 x^{20}+x^{24}+2 x^{26} \\
& e_{3}=\bar{f}_{1}\left(u_{3}^{\prime}\right)=1+2\left(\sum_{i=10}^{17} x^{i}\right)+\left(\sum_{i=18}^{26} x^{i}\right) \\
& e_{4}=\bar{f}_{1}\left(u_{4}^{\prime}\left(u_{1}^{\prime}\right)^{2}\left(u_{3}^{\prime}\right)^{2}\right)=1+2 x^{14}+x^{15}+x^{16}+2 x^{17}+x^{18}+x^{20}+2 x^{21}+2 x^{23}+2 x^{25} \\
& e_{5}=\bar{f}_{1}\left(u_{5}^{\prime}\left(u_{1}^{\prime}\right)^{2}\left(u_{2}^{\prime}\right)^{2}\left(u_{3}^{\prime}\right) u_{4}^{\prime}\right)=1+2 x^{16}+2 x^{17}+x^{18}+x^{25}
\end{aligned}
$$




$$
\begin{aligned}
e_{6} & =\bar{f}_{1}\left(u_{6}^{\prime}\left(u_{1}^{\prime} u_{2}^{\prime}\right)^{6} u_{3}^{\prime}\right)=1+2 x^{20}+x^{21}+2 x^{23}+2 x^{24}+2 x^{26} \\
e_{7} & =\bar{f}_{1}\left(u_{7}^{\prime}\left(u_{1}^{\prime}\right)^{7}\left(u_{3}^{\prime}\right)^{2} u_{4}^{\prime}\left(u_{6}^{\prime}\right)^{2}\right)=1+2 x^{22}+2 x^{23}+2 x^{24}+x^{25}+2 x^{26} \\
e_{8} & =\bar{f}_{1}\left(u_{8}^{\prime}\left(u_{1}^{\prime}\right)^{4}\left(u_{2}^{\prime}\right)^{7}\left(u_{3}^{\prime}\right)\left(u_{4}^{\prime}\right)^{4} u_{5}^{\prime}\left(u_{6}^{\prime}\right) u_{7}^{\prime}\right)=1+x^{24}+2 x^{26} \\
e_{9} & =\bar{f}_{1}\left(u_{9}\right) e_{7}^{2}=\bar{f}_{1}\left(\left(u_{1}^{\prime}\right)^{5}\left(u_{3}^{\prime}\right)\left(u_{4}^{\prime}\right)^{2}\left(u_{6}^{\prime}\right)^{2}\left(u_{7}\right)^{2} u_{9}\right)=1 \\
e_{10} & =\bar{f}_{1}\left(u_{10}\right) e_{7} e_{8}^{2}=\bar{f}_{1}\left(\left(u_{1}^{\prime}\right)^{6}\left(u_{2}^{\prime}\right)^{5}\left(u_{3}^{\prime}\right)\left(u_{5}^{\prime}\right)^{2}\left(u_{6}^{\prime}\right)\left(u_{7}^{\prime}\right)^{3}\left(u_{8}^{\prime}\right)^{2}\left(u_{10}\right)\right)=1
\end{aligned}
$$

Então temos $\operatorname{Im}\left(\bar{f}_{1}\right)=\left\langle e_{1}\right\rangle \times\left\langle e_{2}\right\rangle \times \cdots\left\langle e_{7}\right\rangle \times\left\langle e_{8}\right\rangle$. Seja o $\left(e_{i}\right)$ a ordem de $e_{i}$. Assim:

$$
\begin{aligned}
o\left(e_{3}\right)=o\left(e_{4}\right)=o\left(e_{5}\right)=o\left(e_{6}\right)=o\left(e_{7}\right)=o\left(e_{8}\right) & =3 \\
o\left(e_{1}\right)=o\left(e_{2}\right) & =3^{2} .
\end{aligned}
$$

$\operatorname{Logo}\left|\operatorname{Im}\left(\bar{f}_{1}\right)\right|=o\left(e_{1}\right) \cdot o\left(e_{2}\right) \cdots o\left(e_{8}\right)=3^{10}$.

Seja $w_{i} \in U_{1}\left(\mathbb{Z} C_{27}\right)$ tal que $\bar{f}_{1}\left(w_{i}\right)=e_{i}$, que encontramos acima. Estes elementos escritos em função de $u_{1}^{\prime}, u_{2}^{\prime}, \cdots, u_{8}^{\prime}, u_{9}$ e $u_{10}$ fornecem a matriz

$$
\left(\begin{array}{llllllllll}
1 & 0 & 0 & 0 & 0 & 0 & 0 & 0 & 0 & 0 \\
1 & 1 & 0 & 0 & 0 & 0 & 0 & 0 & 0 & 0 \\
0 & 0 & 1 & 0 & 0 & 0 & 0 & 0 & 0 & 0 \\
2 & 0 & 2 & 1 & 0 & 0 & 0 & 0 & 0 & 0 \\
2 & 2 & 1 & 1 & 1 & 0 & 0 & 0 & 0 & 0 \\
6 & 6 & 1 & 0 & 0 & 1 & 0 & 0 & 0 & 0 \\
7 & 0 & 2 & 1 & 0 & 2 & 1 & 0 & 0 & 0 \\
4 & 7 & 1 & 4 & 1 & 1 & 1 & 1 & 0 & 0 \\
5 & 0 & 1 & 2 & 0 & 2 & 2 & 0 & 1 & 0 \\
6 & 5 & 1 & 0 & 2 & 1 & 3 & 2 & 0 & 1
\end{array}\right)
$$

que é unitriangular inferior e por isso é inversível.

$\operatorname{Logo}\left\langle w_{1}, w_{2}, \cdots, w_{9}, w_{10}\right\rangle=\left\langle u_{1}^{\prime}, u_{2}^{\prime}, \cdots, u_{8}^{\prime}, u_{9}, u_{10}\right\rangle=U_{*}\left(\mathbb{Z} C_{27}\right)$.

Como $\operatorname{ker}\left(\bar{f}_{1}\right) \subseteq U_{*}\left(\mathbb{Z} C_{27}\right)$, se $\alpha \in \operatorname{ker}\left(\bar{f}_{1}\right)$, então $\alpha=w_{1}^{r_{1}} \cdot w_{2}^{r_{2}} \cdots w_{10}^{r_{10}} \cdot$ Logo

$\alpha \in \operatorname{ker}\left(\bar{f}_{1}\right) \Leftrightarrow \bar{f}_{1}(\alpha)=\bar{f}_{1}\left(w_{1}^{r_{1}}\right) \cdots \bar{f}_{1}\left(w_{8}^{r_{8}}\right) \bar{f}_{1}\left(w_{9}^{r_{9}}\right) \cdot \bar{f}_{1}\left(w_{10}^{r_{10}}\right)=1 \Leftrightarrow \bar{f}_{1}(\alpha)=\bar{f}_{1}\left(w_{1}^{r_{1}}\right) \cdots \bar{f}_{1}\left(w_{8}^{r_{8}}\right)=1$. 
Temos $\left\{e_{1}=\bar{f}_{1}\left(w_{1}\right), e_{2}=\bar{f}_{1}\left(w_{2}\right), \cdots, e_{8}=\bar{f}_{1}\left(w_{8}\right)\right\}$ multiplicativamente independente, portanto,

$$
\bar{f}_{1}(\alpha)=1 \Leftrightarrow \bar{f}_{1}\left(w_{i}\right)^{r_{i}}=1 \Leftrightarrow o\left(e_{i}\right) \text { divide } r_{i} \text {, para todo } 1 \leq i \leq 10 .
$$

Como $w_{9}, w_{10} \in \operatorname{ker}\left(\bar{f}_{1}\right)$, temos o $\left(e_{9}\right)=o\left(e_{10}\right)=1$ e então

$$
\operatorname{ker}\left(\bar{f}_{1}\right)=\left\langle w_{1}^{o\left(e_{1}\right)}, w_{2}^{o\left(e_{2}\right)}, \cdots, w_{8}^{o\left(e_{8}\right)}, w_{9}, w_{10}\right\rangle
$$

ou seja,

$$
\begin{gathered}
\operatorname{ker}\left(\bar{f}_{1}\right)=\left\langle\left\{\left(u_{1}^{\prime}\right)^{9},\left(u_{2}^{\prime} u_{1}^{\prime}\right)^{9},\left(u_{3}^{\prime}\right)^{3},\left(u_{4}^{\prime}\left(u_{1}^{\prime}\right)^{2}\left(u_{3}^{\prime}\right)^{2}\right)^{3},\left(u_{5}^{\prime}\left(u_{1}^{\prime}\right)^{2}\left(u_{2}^{\prime}\right)^{2}\left(u_{3}^{\prime}\right) u_{4}^{\prime}\right)^{3},\left(u_{6}^{\prime}\left(u_{1}^{\prime} u_{2}^{\prime}\right)^{6} u_{3}^{\prime}\right)^{3},\right.\right. \\
\left(u_{7}^{\prime}\left(u_{1}^{\prime}\right)^{7}\left(u_{3}^{\prime}\right)^{2} u_{4}^{\prime}\left(u_{6}^{\prime}\right)^{2}\right)^{3},\left(u_{8}^{\prime}\left(u_{1}^{\prime}\right)^{4}\left(u_{2}^{\prime}\right)^{7}\left(u_{3}^{\prime}\right)\left(u_{4}^{\prime}\right)^{4} u_{5}^{\prime}\left(u_{6}^{\prime}\right) u_{7}^{\prime}\right)^{3},\left(u_{1}^{\prime}\right)^{5}\left(u_{3}^{\prime}\right)\left(u_{4}^{\prime}\right)^{2}\left(u_{6}^{\prime}\right)^{2}\left(u_{7}\right)^{2} u_{9}, \\
\left.\left.\left(u_{1}^{\prime}\right)^{6}\left(u_{2}^{\prime}\right)^{5}\left(u_{3}^{\prime}\right)\left(u_{5}^{\prime}\right)^{2}\left(u_{6}^{\prime}\right)\left(u_{7}^{\prime}\right)^{3}\left(u_{8}^{\prime}\right)^{2}\left(u_{10}\right)\right\}\right\rangle .
\end{gathered}
$$

Exemplo 4.4. Vamos encontrar $\operatorname{ker}\left(\bar{\pi}_{1}\right)$ no caso $\mathbb{Z} C_{5^{2}}$.

Este é um caso mais simples pois ker $\left(\bar{\pi}_{1}\right)$ tem posto 1 . Se

$$
w=1+a_{0} \widehat{\left(g^{5}\right)}+a_{1} g \widehat{\left(g^{5}\right)}+a_{2} g^{2} \widehat{\left(g^{5}\right)}+a_{3} g^{3} \widehat{\left(g^{5}\right)}+a_{4} g^{4} \widehat{\left(g^{5}\right)} \in \operatorname{ker}\left(\bar{\pi}_{1}\right)
$$

onde $\widehat{\left(g^{5}\right)}=1+g^{5}+g^{10}+g^{15}+g^{20}$, ent $\tilde{a} o \pi_{2}(w)=1+5\left(a_{0}+a_{1} h+a_{2} h^{2}+a_{3} h^{3}+a_{4} h^{4}\right)$.

Temos $U_{*}\left(\mathbb{Z} C_{5}\right)=\left\langle u_{1}=-1+h+h^{4}\right\rangle$ e se $x=h-1$, então $\bar{f}_{1}\left(u_{1}\right)=1+x^{2}+4 x^{3}+x^{4}$ e $\bar{f}_{1}\left(u_{1}^{5}\right)=1$. Logo $\operatorname{Im}\left(\bar{f}_{1}\right)=\left\langle 1+x^{2}+4 x^{3}+x^{4}\right\rangle$ e $\operatorname{ker}\left(\bar{f}_{1}\right)=\left\langle u_{1}^{5}\right\rangle$ onde $u_{1}^{5}=1+5\left(-10+8 h-3 h^{2}-3 h^{3}+8 h^{4}\right)$.

Igualamos $\pi_{2}(w)=u_{1}^{5}$ e encontramos $w \in \operatorname{ker}\left(\bar{\pi}_{1}\right)$.

$$
u_{1}^{5}=1+5\left(-10+8 h-3 h^{2}-3 h^{3}+8 h^{4}\right)=\pi_{2}(w)=1+5\left(a_{0}+a_{1} h+a_{2} h^{2}+a_{3} h^{3}+a_{4} h^{4}\right)
$$

Logo $a_{0}=-10, a_{1}=a_{4}=8$ e $a_{2}=a_{3}=-3$ e assim

$$
w=1-10 \widehat{\left(g^{5}\right)}+8 g \widehat{\left(g^{5}\right)}-3 g^{2} \widehat{\left(g^{5}\right)}-3 g^{3} \widehat{\left(g^{5}\right)}+8 g^{4} \widehat{\left(g^{5}\right)}
$$

Portanto, $\operatorname{ker}\left(\bar{\pi}_{1}\right)=\left\langle 1-10 \widehat{\left(g^{5}\right)}+8 g \widehat{\left(g^{5}\right)}-3 g^{2} \widehat{\left(g^{5}\right)}-3 g^{3} \widehat{\left(g^{5}\right)}+8 g^{4} \widehat{\left(g^{5}\right)}\right\rangle$. 
Exemplo 4.5. Caso $\mathbb{Z} C_{7^{2}}$.

Sejam $C_{7^{2}}=\langle g\rangle, C_{7}=\langle h\rangle$ e $x=h-1$.

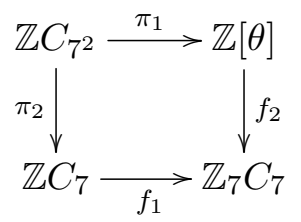

Vamos encontrar a ordem de $\operatorname{Im}\left(\bar{f}_{1}\right)$. Temos $U_{*}\left(\mathbb{Z} C_{7}\right)=\left\langle u_{1}, u_{2}\right\rangle$, onde $u_{1}=-1+h+h^{6} e$ $u_{2}=1-h^{2}+h^{3}+h^{4}-h^{5}$.

Aplicando $\bar{f}_{1}$ em $u_{1}$ e $u_{2}$ e depois substituindo $h=x+1$ em cada uma das imagens, obtemos:

$$
\begin{aligned}
& \bar{f}_{1}\left(u_{1}\right)=-\overline{1}+h+h^{6}=1+x^{2}+\overline{6} x^{3}+x^{4}+\overline{6} x^{5}+x^{6} \\
& \bar{f}_{1}\left(u_{2}\right)=\overline{1}-\overline{1} h^{2}+h^{3}+h^{4}-\overline{1} h^{5}=1+\overline{5} x^{2}+\overline{2} x^{3}+\overline{3} x^{4}+\overline{6} x^{5} .
\end{aligned}
$$

Vamos agora encontrar um conjunto multiplicativamente independente que gera $\operatorname{Im}\left(\bar{f}_{1}\right)$.

$$
\begin{aligned}
& e_{1}=\bar{f}_{1}\left(u_{1}\right)=\overline{1}+x^{2}+\overline{6} x^{3}+x^{4}+\overline{6} x^{5}+x^{6} \\
& e_{2}=\bar{f}_{1}\left(u_{2} \cdot u_{1}^{2}\right)=\overline{1}+\overline{2} x^{4}+\overline{3} x^{5}+x^{6}
\end{aligned}
$$

Logo $\operatorname{Im}\left(\bar{f}_{1}\right)=\left\langle e_{1}\right\rangle \times\left\langle e_{2}\right\rangle$. Como a ordem de $e_{1}$ e de e e são iguais a 7 , então $\left|\operatorname{Im}\left(\bar{f}_{1}\right)\right|=7^{2}$.

Da mesma maneira do exemplo 4.3, encontramos $\left\langle u_{1}, u_{1}^{2} \cdot u_{2}\right\rangle=\left\langle u_{1}, u_{2}\right\rangle=U_{*}\left(\mathbb{Z} C_{7}\right)$, $\operatorname{logo}$ $\operatorname{ker}\left(\bar{f}_{1}\right)=\left\langle u_{1}^{7},\left(u_{1}^{2} \cdot u_{2}\right)^{7}\right\rangle$.

Exemplo 4.6. Caso $\mathbb{Z} C_{2^{4}}$.

Sejam $C_{2^{4}}=\langle g\rangle$ e $C_{8}=\langle h\rangle$. Se $w \in \operatorname{ker}\left(\bar{\pi}_{1}\right)$, então

$$
w=1+a_{0}\left(1+g^{8}\right)+a_{1} g\left(1+g^{8}\right)+a_{2} g^{2}\left(1+g^{8}\right)+a_{3} g^{3}\left(1+g^{8}\right)+\cdots+a_{7} g^{7}\left(1+g^{8}\right),
$$

$e$

$$
\pi_{2}(w)=1+2\left(a_{0}+a_{1} h+a_{2} h^{2}+a_{3} h^{3}+a_{4} h^{4}+a_{5} h^{5}+a_{6} h^{6}+a_{7} h^{7}\right) .
$$

Temos $U_{1}\left(\mathbb{Z} C_{8}\right)=\left\langle\left\{h,-1-h-h^{2}+h^{4}+2 h^{5}+h^{6}\right\}\right\rangle$. Esta base para $U_{1}\left(\mathbb{Z} C_{8}\right)$ pode ser encontrada em [27]. 
Temos $u_{1}=-1-h-h^{2}+h^{4}+2 h^{5}+h^{6}=h\left(-1-h+h^{3}+2 h^{4}+h^{5}-h^{7}\right)=h u_{1}^{\prime}$. Observe que

$$
\begin{aligned}
u_{1}^{\prime} & =1+\left(-2+2 h^{4}\right)-\left(h+h^{7}-2\right)+\left(h^{3}+h^{5}-2\right) \\
& =1-\left(h^{4}-1\right)^{2}+\left((h-1) \cdot\left(h^{7}-1\right)\right)-\left(\left(h^{3}-1\right) \cdot\left(h^{5}-1\right)\right) \equiv 1\left(\bmod \left(\Delta C_{8}\right)^{2}\right) .
\end{aligned}
$$

Portanto, iremos utilizar a unidade $u_{1}^{\prime} \in U_{2}\left(\mathbb{Z} C_{8}\right)$ e, deste modo, $U_{1}\left(\mathbb{Z} C_{8}\right)=C_{8} \times\left\langle u_{1}^{\prime}\right\rangle$. Para encontrar os coeficientes $a_{0}, a_{1}, a_{2}, \cdots, a_{7}$ de $w$ basta fazer $\left(u_{1}^{\prime}\right)^{2}=1+2\left(4+3 h-3 h^{3}-4 h^{4}-3 h^{5}+3 h^{7}\right)=\pi_{2}(w)=1+2\left(a_{0}+a_{1} h+a_{2} h^{2}+\cdots+a_{6} h^{6}+a_{7} h^{7}\right)$.

Deste modo obtemos $a_{0}=4, a_{1}=a_{7}=3, a_{2}=a_{6}=0, a_{3}=a_{5}=-3$ e $a_{4}=-4$. Então

$$
w=1+4\left(1+g^{8}\right)+3 g\left(1+g^{8}\right)-3 g^{3}\left(1+g^{8}\right)-4 g^{4}\left(1+g^{8}\right)-3 g^{5}\left(1+g^{8}\right)+3 g^{7}\left(1+g^{8}\right) .
$$

Assim $w \in \operatorname{ker}\left(\bar{\pi}_{1}\right)$ e, portanto, $\operatorname{ker}\left(\bar{\pi}_{1}\right)=\langle w\rangle$.

Exemplo 4.7. Caso $\mathbb{Z} C_{2^{5}}$.

Sejam $C_{2^{5}}=\langle g\rangle, C_{2^{4}}=\langle h\rangle$ e $x=h-1$.

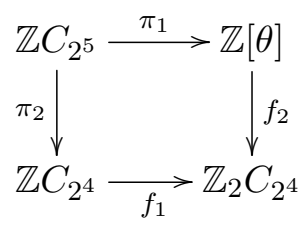

Vamos encontrar um conjunto multiplicativamente independente que gere $\operatorname{Im}\left(\bar{f}_{1}\right)$.

Temos $U_{2}\left(\mathbb{Z} C_{16}\right)=\left\langle u_{1}^{\prime}, u_{2}^{\prime}, u_{3}^{\prime}, u_{4}\right\rangle$ onde $u_{1}, u_{2}$ e $u_{3}$ são as unidades de Hoechsmann em $\mathbb{Z} C_{16}$ e $u_{4}$ é a unidade que encontramos no exemplo anterior. $u_{1}^{\prime}, u_{2}^{\prime}$ e $u_{3}^{\prime}$ são as unidades em $U_{2}\left(\mathbb{Z} C_{16}\right)$ que encontramos a partir de $u_{1}, u_{2}$ e $u_{3}$.

$$
\begin{aligned}
u_{1} & =\left(1+h^{3}+h^{2 \cdot 3}+\cdots+h^{(10) 3}\right)\left(1+h^{3}+h^{2 \cdot 3}\right)-2 \widehat{h} \\
& =h^{2}\left[1-\left(h^{2}+h^{14}\right)+\left(h^{3}+h^{13}\right)+\left(h^{4}+h^{12}\right)-2\left(h^{5}+h^{11}\right)+\left(h^{6}+h^{10}\right)+\left(h^{7}+h^{9}\right)-2 h^{8}\right]=h^{2} \cdot u_{1}^{\prime} \\
u_{2} & =\left(1+h^{3}+h^{2 \cdot 3}+\cdots+h^{(10) 3}\right)\left(1+h^{9}+h^{2 \cdot 9}\right)-2 \widehat{h} \\
& =h^{8}\left[1-\left(h^{2}+h^{14}\right)+\left(h^{3}+h^{13}\right)-\left(h^{5}+h^{11}\right)+\left(h^{6}+h^{10}\right)\right]=h^{8} \cdot u_{2}^{\prime}
\end{aligned}
$$




$$
\begin{aligned}
u_{3} & =\left(1+h^{3}+h^{2 \cdot 3}+\cdots+h^{(10) 3}\right)\left(1+h^{11}+h^{6}\right)-2 \widehat{h} \\
& =h^{10}\left[-1+\left(h+h^{15}\right)-\left(h^{3}+h^{13}\right)+\left(h^{4}+h^{12}\right)\right]=h^{10} \cdot u_{3}^{\prime} \\
u_{4} & =1+4\left(1+h^{8}\right)+3 h\left(1+h^{8}\right)-3 h^{3}\left(1+h^{8}\right)-4 h^{4}\left(1+h^{8}\right)-3 h^{5}\left(1+h^{8}\right)+3 h^{7}\left(1+h^{8}\right) \\
& =5+3\left(h+h^{15}\right)-3\left(h^{3}+h^{13}\right)-4\left(h^{4}+h^{12}\right)-3\left(h^{5}+h^{11}\right)+3\left(h^{7}+h^{9}\right)+4 h^{8} .
\end{aligned}
$$

Aplicando $\bar{f}_{1}$ em $u_{1}^{\prime}, u_{2}^{\prime}, u_{3}^{\prime}$ e $u_{4}$ e depois substituindo $h=x+1$ em cada uma das imagens, obtemos:

$$
\begin{aligned}
& \bar{f}_{1}\left(u_{1}^{\prime}\right)=1+x^{6}+x^{7}+x^{8}+x^{12}+x^{13}+x^{14} \\
& \bar{f}_{1}\left(u_{2}^{\prime}\right)=1+x^{10}+x^{11}+x^{13}+x^{14} \\
& \bar{f}_{1}\left(u_{3}^{\prime}\right)=1+x^{6}+x^{7}+x^{8}+x^{10}+x^{11}+x^{12}+x^{14}+x^{15} \\
& \bar{f}_{1}\left(u_{4}\right)=1+x^{14}+x^{15}
\end{aligned}
$$

Vamos encontrar um conjunto multiplicativamente independente que gera $\operatorname{Im}\left(\bar{f}_{1}\right)$. Temos o seguinte: $\bar{f}_{1}\left(\left(u_{1}^{\prime}\right)^{-1} u_{2}^{\prime} u_{3}^{\prime} u_{4}\right)=1$. Como $e_{1}=\bar{f}_{1}\left(u_{1}^{\prime}\right), e_{2}=\bar{f}_{1}\left(u_{2}^{\prime}\right)$ e $e_{3}=\bar{f}_{1}\left(u_{4}\right)$ são multiplicativamente independentes e $\bar{f}_{1}\left(\left(u_{1}^{\prime}\right)^{-1} u_{2}^{\prime} u_{3}^{\prime} u_{4}\right)=1$, então:

$$
\operatorname{Im}\left(\bar{f}_{1}\right)=\left\langle e_{1}\right\rangle \times\left\langle e_{2}\right\rangle \times\left\langle e_{3}\right\rangle .
$$

A ordem de $e_{1}$ é 4 e as ordens de $e_{2}$ e e e são iguais a 2, então $\left|\operatorname{Im}\left(\bar{f}_{1}\right)\right|=4 \cdot 2 \cdot 2=2^{4}$. Assim como no exemplo 4.3, encontramos $\left\langle u_{1}, u_{2}, u_{4},\left(u_{1}^{\prime}\right)^{-1} u_{2}^{\prime} u_{3}^{\prime} u_{4}\right\rangle=\left\langle u_{1}, u_{2}, u_{3}, u_{4}\right\rangle=U_{*} \mathbb{Z} C_{2^{4}}$ e então $\operatorname{ker}\left(\bar{f}_{1}\right)=\left\langle\left(u_{1}^{\prime}\right)^{4},\left(u_{2}^{\prime}\right)^{2},\left(u_{4}^{\prime}\right)^{2},\left(u_{1}^{\prime}\right)^{-1} u_{2}^{\prime} u_{3}^{\prime} u_{4}\right\rangle$.

Vamos agora procurar uma base para $\operatorname{ker}\left(\bar{\pi}_{1}\right)$. Se $v_{1}, v_{2}, v_{3}, v_{4} \in \operatorname{ker}\left(\bar{\pi}_{1}\right)$ são os geradores de $\operatorname{ker}\left(\bar{\pi}_{1}\right)$, então $\pi_{2}\left(v_{i}\right)=1+2 w_{i}$, com $w_{i} \in \mathbb{Z} C_{16}$. Igualamos cada um dos geradores de $\operatorname{ker}\left(\bar{f}_{1}\right)$ com $\pi_{2}\left(v_{i}\right)=1+2 w_{i}$, e deste modo encontramos $v_{i}$ 's.

$$
\begin{aligned}
\text { Seja } v_{i}=1+ & \left(1+g^{16}\right) \cdot\left(a_{0}+a_{1} g+a_{2} g^{2}+\cdots+a_{15} g^{15}\right) e \\
\left(u_{1}^{\prime}\right)^{4}= & 1+2\left(1428-570\left(h+h^{15}\right)-959\left(h^{2}+h^{14}\right)+1304\left(h^{3}+h^{13}\right)-72\left(h^{4}+h^{12}\right)+\right. \\
& \left.-1202\left(h^{5}+h^{11}\right)+959\left(h^{6}+h^{10}\right)+468\left(h^{7}+h^{9}\right)-1284 h^{8}\right) \\
\left(u_{1}^{\prime}\right)^{-4}= & 1+2\left(84+42\left(h+h^{15}\right)+7\left(h^{2}+h^{14}\right)-56\left(h^{3}+h^{13}\right)-72\left(h^{4}+h^{12}\right)+\right. \\
& \left.-46\left(h^{5}+h^{11}\right)-7\left(h^{6}+h^{10}\right)+60\left(h^{7}+h^{9}\right)+60 h^{8}\right) \\
= & \pi_{2}\left(v_{1}\right)=1+2\left(a_{0}+a_{1} h+a_{2} h^{2}+\cdots+a_{15} h^{15}\right)
\end{aligned}
$$




$$
\begin{aligned}
\left(u_{2}^{\prime}\right)^{2}= & 1+2\left(4-2\left(h+h^{15}\right)-2\left(h^{2}+h^{14}\right)+3\left(h^{3}+h^{13}\right)-3\left(h^{5}+h^{11}\right)+2\left(h^{6}+h^{10}\right)+\right. \\
& \left.+2\left(h^{7}+h^{9}\right)-4 h^{8}\right)=\pi_{2}\left(v_{2}\right)=1+2\left(a_{0}+a_{1} h+a_{2} h^{2}+\cdots+a_{15} h^{15}\right) \\
\left(u_{4}\right)^{2}= & 1+2\left(72+51\left(h+h^{15}\right)-51\left(h^{3}+h^{13}\right)-72\left(h^{4}+h^{12}\right)-51\left(h^{5}+h^{11}\right)+\right. \\
& \left.+51\left(h^{7}+h^{9}\right)+72 h^{8}\right)=\pi_{2}\left(v_{3}\right)=1+2\left(a_{0}+a_{1} h+\cdots+a_{15} h^{15}\right) \\
\left(u_{1}^{\prime}\right)^{-1} u_{2}^{\prime} u_{3}^{\prime} u_{4}= & 1+2\left(3\left(h+h^{15}\right)-\left(h^{2}+h^{14}\right)-\left(h^{3}+h^{13}\right)-2\left(h^{4}+h^{12}\right)+\right. \\
& \left.-2\left(h^{5}+h^{11}\right)+\left(h^{6}+h^{10}\right)+4 h^{8}\right)=\pi_{2}\left(v_{4}\right)=1+2\left(a_{0}+a_{1} h+\cdots+a_{15} h^{15}\right) .
\end{aligned}
$$

Logo

$$
\begin{aligned}
v_{1}= & 1+\left(1+g^{16}\right) \cdot\left[84+42\left(g+g^{15}\right)+7\left(g^{2}+g^{14}\right)-56\left(g^{3}+g^{13}\right)-72\left(g^{4}+g^{12}\right)+\right. \\
& \left.-46\left(g^{5}+g^{11}\right)-7\left(g^{6}+g^{10}\right)+60\left(g^{7}+g^{9}\right)+60 g^{8}\right] \\
v_{2}= & 1+\left(1+g^{16}\right) \cdot\left[4-2\left(g+g^{15}\right)-2\left(g^{2}+g^{14}\right)+3\left(g^{3}+g^{13}\right)-3\left(g^{5}+g^{11}\right)+2\left(g^{6}+g^{10}\right)+\right. \\
& \left.+2\left(g^{7}+g^{9}\right)-4 g^{8}\right] \\
v_{3}= & 1+\left(1+g^{16}\right) \cdot\left[72+51\left(g+g^{15}\right)-51\left(g^{3}+g^{13}\right)-72\left(g^{4}+g^{12}\right)-51\left(g^{5}+g^{11}\right)+\right. \\
& \left.+51\left(g^{7}+g^{9}\right)+72 g^{8}\right] \\
v_{4}= & 1+\left(1+g^{16}\right) \cdot\left[3\left(g+g^{15}\right)-\left(g^{2}+g^{14}\right)-\left(g^{3}+g^{13}\right)-2\left(g^{4}+g^{12}\right)-2\left(g^{5}+g^{11}\right)+\right. \\
& \left.+\left(g^{6}+g^{10}\right)+4 g^{8}\right]
\end{aligned}
$$

Portanto, $\operatorname{ker}\left(\bar{\pi}_{1}\right)=\left\langle v_{1}, v_{2}, v_{3}, v_{4}\right\rangle$.

Exemplo 4.8. Caso $\mathbb{Z} C_{2^{6}}$.

Sejam $C_{2^{6}}=\langle g\rangle, C_{2^{5}}=\langle h\rangle$ e $x=h-1$.

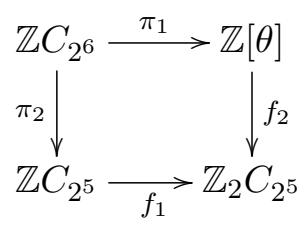

Vamos encontrar um conjunto multiplicativamente independente que gere $\operatorname{Im}\left(\bar{f}_{1}\right)$.

Temos $U_{1}\left(\mathbb{Z} C_{2^{5}}\right)=C_{2^{5}} \times\left\langle u_{1}^{\prime}, u_{2}^{\prime}, \cdots, u_{7}^{\prime}, u_{8}, u_{9}, u_{10}, u_{11}\right\rangle$, onde $u_{1}, u_{2}, \cdots u_{6}$ e $u_{7}$ são as unidades de Hoechsmann em $\mathbb{Z} C_{2^{5}}$ e $u_{8}, u_{9}, u_{10}$ e $u_{11}$ são as unidades que encontramos no exemplo anterior. 
$u_{1}^{\prime}, u_{2}^{\prime} \cdots, u_{6}^{\prime}$ e $u_{7}^{\prime}$ são as unidades de $U_{2}\left(\mathbb{Z} C_{32}\right)$ que encontramos a partir de $u_{1}, u_{2} \cdots, u_{6}$ e $u_{7}$.

$$
\begin{aligned}
& u_{1}=\left(1+h^{3}+h^{2 \cdot 3}+\cdots+h^{(10) 3}\right)\left(1+h^{3}+h^{2 \cdot 3}\right)-\widehat{h} \\
& =h^{2}\left[-1+2 h^{16}+\left(h+h^{31}\right)-\left(h^{3}+h^{29}\right)+2\left(h^{4}+h^{28}\right)-\left(h^{5}+h^{27}\right)-\left(h^{6}+h^{26}\right)+\right. \\
& +2\left(h^{7}+h^{25}\right)-\left(h^{8}+h^{24}\right)-\left(h^{9}+h^{23}\right)+2\left(h^{10}+h^{22}\right)-\left(h^{11}+h^{21}\right)-\left(h^{12}+h^{20}\right)+ \\
& \left.+2\left(h^{13}+h^{19}\right)-\left(h^{14}+h^{18}\right)-\left(h^{15}+h^{17}\right)\right]=h^{2} \cdot u_{1}^{\prime} \\
& u_{2}=\left(1+h^{3}+h^{2 \cdot 3}+\cdots+h^{(10) 3}\right)\left(1+h^{9}+h^{2 \cdot 9}\right)-\widehat{h} \\
& =h^{8}\left[-1+\left(h+h^{31}\right)-\left(h^{3}+h^{29}\right)+\left(h^{4}+h^{28}\right)-\left(h^{6}+h^{26}\right)+\left(h^{7}+h^{25}\right)-\left(h^{9}+h^{23}\right)+\right. \\
& \left.+2\left(h^{10}+h^{22}\right)-\left(h^{11}+h^{21}\right)-\left(h^{12}+h^{20}\right)+2\left(h^{13}+h^{19}\right)-\left(h^{14}+h^{18}\right)-\left(h^{15}+h^{17}\right)+2 h^{16}\right] \\
& =h^{8} \cdot u_{2}^{\prime} \\
& u_{3}=\left(1+h^{3}+h^{2 \cdot 3}+\cdots+h^{(10) 3}\right)\left(1+h^{27}+h^{22}\right)-\widehat{h} \\
& =h^{26}\left[-1+\left(h+h^{31}\right)-\left(h^{3}+h^{29}\right)+\left(h^{4}+h^{28}\right)\right]=h^{26} \cdot u_{3}^{\prime} \\
& u_{4}=\left(1+h^{3}+h^{2 \cdot 3}+\cdots+h^{(10) 3}\right)\left(1+h^{17}+h^{2}\right)-\widehat{h} \\
& =h^{16}\left[-1+\left(h+h^{31}\right)-\left(h^{3}+h^{29}\right)+\left(h^{4}+h^{28}\right)-\left(h^{6}+h^{26}\right)+\left(h^{7}+h^{25}\right)-\left(h^{9}+h^{23}\right)+\right. \\
& \left.+\left(h^{10}+h^{22}\right)-\left(h^{12}+h^{20}\right)+\left(h^{13}+h^{19}\right)-\left(h^{15}+h^{17}\right)+2 h^{16}\right]=h^{16} \cdot u_{4}^{\prime} \\
& u_{5}=\left(1+h^{3}+h^{2 \cdot 3}+\cdots+h^{(10) 3}\right)\left(1+h^{19}+h^{6}\right)-\widehat{h} \\
& =h^{18}\left[1-\left(h^{2}+h^{30}\right)+\left(h^{3}+h^{29}\right)-\left(h^{5}+h^{27}\right)+\left(h^{6}+h^{26}\right)-\left(h^{8}+h^{24}\right)+\left(h^{9}+h^{23}\right)+\right. \\
& \left.-\left(h^{11}+h^{21}\right)+\left(h^{12}+h^{20}\right)\right]=h^{18} \cdot u_{5}^{\prime} \\
& u_{6}=\left(1+h^{3}+h^{2 \cdot 3}+\cdots+h^{(10) 3}\right)\left(1+h^{25}+h^{18}\right)-\widehat{h} \\
& =h^{24}\left[1-\left(h^{2}+h^{30}\right)+\left(h^{3}+h^{29}\right)-\left(h^{5}+h^{27}\right)+\left(h^{6}+h^{26}\right)\right]=h^{24} \cdot u_{6}^{\prime} \\
& u_{7}=\left(1+h^{3}+h^{2 \cdot 3}+\cdots+h^{(10) 3}\right)\left(1+h^{11}+h^{22}\right)-\widehat{h} \\
& =h^{10}\left[-1+\left(h+h^{31}\right)-\left(h^{3}+h^{29}\right)+\left(h^{4}+h^{28}\right)-\left(h^{6}+h^{26}\right)+\left(h^{7}+h^{25}\right)-\left(h^{9}+h^{23}\right)+\right. \\
& \left.+\left(h^{10}+h^{22}\right)\right]=h^{10} \cdot u_{7}^{\prime}
\end{aligned}
$$

Vamos aplicar $\bar{f}_{1}$ em cada um dos elementos $u_{1}^{\prime}, u_{2}^{\prime}, \cdots, u_{9}, u_{10}, u_{11}$ e substituir $h=x+1$ em cada uma destas imagens. Neste caso $\bar{f}_{1}\left(u_{1}^{\prime}\right), \bar{f}_{1}\left(u_{2}^{\prime}\right), \cdots \bar{f}_{1}\left(u_{11}\right)$ não são multiplicativamente independentes, mas a partir destes elementos encontramos o conjunto $\left\{e_{1}, e_{2}, \cdots, e_{7}\right\}$ que é mul- 
tiplicativamente independente e que gera $\operatorname{Im}\left(\bar{f}_{1}\right)$, dado por:

$$
\begin{aligned}
& e_{1}=\bar{f}_{1}\left(u_{1}^{\prime}\right)=1+x^{6}+x^{7}+x^{8}+x^{12}+x^{13}+x^{14}+x^{18}+x^{19}+x^{20}+x^{24}+x^{25}+x^{26}+x^{30}+x^{31} \\
& e_{2}=\bar{f}_{1}\left(u_{2}^{\prime}\right)=1+x^{10}+x^{11}+x^{13}+x^{14}+x^{24}+x^{25}+x^{27}+x^{28}+x^{30}+x^{31} \\
& e_{3}=\bar{f}_{1}\left(u_{3}^{\prime}\left(u_{1}^{\prime}\right)^{3} u_{2}^{\prime}\right)=1+x^{14}+x^{15}+x^{24}+x^{29}+x^{30}+x^{31} \\
& e_{4}=\bar{f}_{1}\left(u_{4}^{\prime}\right)=1+x^{18}+x^{19}+x^{21}+x^{22}+x^{24}+x^{25}+x^{27}+x^{28}+x^{30}+x^{31} \\
& e_{5}=\bar{f}_{1}\left(u_{5}^{\prime}\left(u_{1}^{\prime}\right)^{3} u_{4}^{\prime}\right)=1+x^{22}+x^{23}+x^{24}+x^{28}+x^{29}+x^{31} \\
& e_{6}=\bar{f}_{1}\left(u_{6}^{\prime}\left(u_{2}^{\prime}\right)^{3} u_{4}^{\prime}\right)=1+x^{26}+x^{27}+x^{28}+x^{29} \\
& e_{7}=\bar{f}_{1}\left(u_{7}^{\prime}\left(u_{1}^{\prime}\right)^{3}\left(u_{2}^{\prime}\right)^{3}\left(u_{3}^{\prime}\right)^{3} u_{4}^{\prime} u_{5}^{\prime} u_{6}^{\prime}\right)=1+x^{30}+x^{31}
\end{aligned}
$$

Então $\operatorname{Im}\left(\bar{f}_{1}\right)=\left\langle e_{1}\right\rangle \times\left\langle e_{2}\right\rangle \times \cdots \times\left\langle e_{7}\right\rangle$ e verifica-se que $\left|\operatorname{Im}\left(\bar{f}_{1}\right)\right|=2^{11}$ pois

$$
\begin{aligned}
e_{1}^{8}=e_{2}^{4} & =e_{4}^{2}=1 \\
e_{3}^{4}=\bar{f}_{1}\left(\left(u_{3}^{\prime}\left(u_{1}^{\prime}\right)^{3} u_{2}^{\prime}\right)^{4}\right) & =\bar{f}_{1}\left(\left(u_{3}^{\prime} u_{1}^{\prime}\right)^{4}\right)=1 \\
e_{5}^{2}=\bar{f}_{1}\left(\left(u_{5}^{\prime}\left(u_{1}^{\prime}\right)^{3} u_{4}^{\prime}\right)^{2}\right) & =\bar{f}_{1}\left(\left(u_{5}^{\prime}\right)^{2}\left(u_{1}^{\prime}\right)^{-2}\right)=1 \\
e_{6}^{2}=\bar{f}_{1}\left(\left(u_{6}^{\prime}\left(u_{2}^{\prime}\right)^{3} u_{4}^{\prime}\right)^{2}\right) & =\bar{f}_{1}\left(\left(u_{6}^{\prime} u_{2}^{\prime}\right)^{2}\right)=1 \\
e_{7}^{2}=\bar{f}_{1}\left(\left(u_{7}^{\prime}\left(u_{1}^{\prime}\right)^{3}\left(u_{2}^{\prime}\right)^{3}\left(u_{3}^{\prime}\right)^{3} u_{4}^{\prime} u_{5}^{\prime} u_{6}^{\prime}\right)^{2}\right) & =f_{1}\left(\left(u_{7}^{\prime}\right)^{2}\left(u_{1}^{\prime}\right)^{-2}\left(u_{2}^{\prime}\right)^{2}\left(u_{3}^{\prime}\right)^{-2}\left(u_{5}^{\prime}\right)^{2}\left(u_{6}^{\prime}\right)^{2}\right)=1 \\
\bar{f}_{1}\left(\left(u_{1}^{\prime}\right)^{6}\left(u_{2}^{\prime}\right)^{2}\left(u_{3}^{\prime}\right)^{2} u_{8}\right) & =\bar{f}_{1}\left(\left(u_{1}^{\prime}\right)^{-2}\left(u_{2}^{\prime}\right)^{2}\left(u_{3}^{\prime}\right)^{2} u_{8}\right)=1 \\
\bar{f}_{1}\left(\left(u_{2}^{\prime}\right)^{3} u_{4}^{\prime} u_{6}^{\prime} u_{9}\right) & =1 \\
\bar{f}_{1}\left(\left(u_{1}^{\prime}\right)^{3}\left(u_{2}^{\prime}\right)^{-1}\left(u_{3}^{\prime}\right)^{3} u_{4}^{\prime} u_{5}^{\prime} u_{6}^{\prime} u_{7}^{\prime} u_{10}\right) & =1 \\
\bar{f}_{1}\left(\left(u_{1}^{\prime}\right)^{-1}\left(u_{2}^{\prime}\right)^{-1} u_{5}^{\prime} u_{6}^{\prime} u_{11}\right) & =1
\end{aligned}
$$

Usando o mesmo raciocínio do exemplo 4.3, temos

$$
\begin{aligned}
& \operatorname{ker}\left(\bar{f}_{1}\right)=\left\langle\left\{\left(u_{1}^{\prime}\right)^{8},\left(u_{2}^{\prime}\right)^{4},\left(u_{3}^{\prime}\left(u_{1}^{\prime}\right)\right)^{4},\left(u_{4}^{\prime}\right)^{2},\left(u_{5}^{\prime}\right)^{2}\left(u_{1}^{\prime}\right)^{-2},\left(u_{6}^{\prime} u_{2}^{\prime}\right)^{2},\left(u_{7}^{\prime}\right)^{2}\left(u_{1}^{\prime}\right)^{-2}\left(u_{2}^{\prime}\right)^{2}\left(u_{3}^{\prime}\right)^{-2}\left(u_{5}^{\prime}\right)^{2}\left(u_{6}^{\prime}\right)^{2}\right.\right. \\
& \left.\left.\quad\left(u_{1}^{\prime}\right)^{-2}\left(u_{2}^{\prime}\right)^{2}\left(u_{3}^{\prime}\right)^{2} u_{8},\left(u_{2}^{\prime}\right)^{3} u_{4}^{\prime} u_{6}^{\prime} u_{9},\left(u_{1}^{\prime}\right)^{3}\left(u_{2}^{\prime}\right)^{-1}\left(u_{3}^{\prime}\right)^{3} u_{4}^{\prime} u_{5}^{\prime} u_{6}^{\prime} u_{7}^{\prime} u_{10},\left(u_{1}^{\prime}\right)^{-1}\left(u_{2}^{\prime}\right)^{-1} u_{5}^{\prime} u_{6}^{\prime} u_{11}\right\}\right\rangle . \\
& \text { e } \operatorname{ker}\left(\bar{\pi}_{1}\right)=\pi_{2}^{-1}\left(\operatorname{ker}\left(\bar{f}_{1}\right)\right) .
\end{aligned}
$$


Exemplo 4.9. Caso $\mathbb{Z} C_{2^{7}}$.

Sejam $C_{2^{7}}=\langle g\rangle, C_{2^{6}}\langle h\rangle$ e $x=h-1$.

Vamos encontrar um conjunto multiplicativamente independente que gere $\operatorname{Im}\left(\bar{f}_{1}\right)$ para verificarmos a sua ordem. Sejam $U_{1}\left(\mathbb{Z} C_{2^{6}}\right)=C_{2^{6}} \times\left\langle u_{1}, u_{2}, \cdots, u_{25}, u_{26}\right\rangle$. Vamos considerar $u_{i}$ 's em $U_{2}\left(\mathbb{Z} C_{2^{6}}\right)$.

Como o mesmo procedimento dos exemplos anteriores temos os seguintes elementos independentes que geram $\operatorname{Im}\left(\bar{f}_{1}\right)$ :

$$
\begin{aligned}
e_{1} & =\bar{f}_{1}\left(u_{1}\right)=1+x^{6}+x^{7}+x^{8}+\cdots \\
e_{2} & =\bar{f}_{1}\left(u_{2}\right)=1+x^{10}+x^{11}+x^{13} \cdots \\
e_{3} & =\bar{f}_{1}\left(u_{3} u_{1}^{3} u_{2}\right)=1+x^{14}+x^{15}+x^{24}+\cdots \\
e_{4} & =\bar{f}_{1}\left(u_{4}\right)=1+x^{18}+x^{19}+x^{21}+\cdots \\
e_{5} & =\bar{f}_{1}\left(u_{5} u_{1}^{3} u_{4}\right)=1+x^{22}+x^{23}+x^{24}+\cdots \\
e_{6} & =\bar{f}_{1}\left(u_{6} u_{2}^{3} u_{4}\right)=1+x^{26}+x^{27}+x^{28}+\cdots \\
e_{7} & =\bar{f}_{1}\left(u_{7} u_{1}^{3} u_{2}^{-1} u_{3}^{3} u_{4}^{-1} u_{5} u_{6}\right)=1+x^{30}+x^{31}+x^{36} \cdots \\
e_{8} & =\bar{f}_{1}\left(u_{8}\right)=1+x^{34}+x^{35}+x^{37}+\cdots \\
e_{9} & =\bar{f}_{1}\left(u_{9} u_{1}^{7} u_{8}\right)=1+x^{38}+x^{39}+x^{45}+\cdots \\
e_{10} & =\bar{f}_{1}\left(u_{10} u_{2}^{7} u_{8}\right)=1+x^{42}+x^{43}+x^{44}+\cdots \\
e_{11} & =\bar{f}_{1}\left(u_{11} u_{1}^{3} u_{2}^{3} u_{3}^{3} u_{8} u_{9} u_{10}\right)=1+x^{46}+x^{47}+x^{48}+\cdots \\
e_{12} & =\bar{f}_{1}\left(u_{12} u_{4}^{3} u_{8}\right)=1+x^{50}+x^{51}+x^{52}+\cdots \\
e_{13} & =\bar{f}_{1}\left(u_{13} u_{1}^{-5} u_{4}^{3} u_{5}^{3} u_{8} u_{9} u_{12}\right)=1+x^{54}+x^{55}+x^{60}+\cdots \\
e_{14} & =\bar{f}_{1}\left(u_{14} u_{2}^{3} u_{4}^{3} u_{6}^{3} u_{8} u_{10} u_{12}\right)=1+x^{58}+x^{59}+x^{60}+x^{61} \\
e_{15} & =\bar{f}_{1}\left(u_{15} u_{1}^{-3} u_{2}^{-3} u_{3}^{3} u_{4} u_{5}^{7} u_{6}^{-1} u_{7}^{3} u_{8} u_{9}^{3} u_{10}^{3} u_{11} u_{12}^{-1} u_{13} u_{14}\right)=1+x^{62}+x^{63}
\end{aligned}
$$

Temos ainda as seguintes combinações:

$$
\begin{aligned}
e_{1}^{16} & =e_{2}^{8}=e_{4}^{4}=e_{8}^{2}=1 \\
e_{3}^{8} & =\bar{f}_{1}\left(u_{3}^{8} u_{1}^{8}\right)=1 \\
e_{5}^{4} & =\bar{f}_{1}\left(u_{5}^{4} u_{1}^{-4}\right)=1 \\
e_{6}^{4} & =\bar{f}_{1}\left(u_{6}^{4} u_{2}^{4}\right)=1 \\
e_{7}^{4} & =\bar{f}_{1}\left(u_{7}^{4} u_{1}^{-4} u_{2}^{-4} u_{3}^{-4} u_{5}^{4} u_{6}\right)=1
\end{aligned}
$$




$$
\begin{aligned}
& e_{9}^{2}=\bar{f}_{1}\left(u_{9}^{2} u_{1}^{-2}\right)=1 \\
& e_{10}^{2}=\bar{f}_{1}\left(u_{10}^{2} u_{2}^{6}\right)=1 \\
& e_{11}^{2}=\bar{f}_{1}\left(u_{11}^{2} u_{1}^{6} u_{2}^{-2} u_{3}^{6} u_{9}^{2} u_{10}^{2}\right)=1 \\
& e_{12}^{2}=\bar{f}_{1}\left(u_{12}^{2} u_{4}^{2}\right)=1 \\
& e_{13}^{2}=\bar{f}_{1}\left(u_{13}^{2} u_{1}^{6} u_{4}^{2} u_{5}^{6} u_{9}^{2} u_{12}^{2}\right)=1 \\
& e_{14}^{2}=\bar{f}_{1}\left(u_{14}^{2} u_{2}^{-2} u_{4}^{2} u_{6}^{-2} u_{10}^{2} u_{12}^{2}\right)=1 \\
& e_{15}^{2}=\bar{f}_{1}\left(u_{15}^{2} u_{1}^{-6} u_{2}^{2} u_{3}^{6} u_{4}^{2} u_{5}^{-2} u_{6}^{-2} u_{7}^{6} u_{9}^{6} u_{10}^{-2} u_{11}^{2} u_{12}^{-2} u_{13}^{2} u_{14}^{2}\right)=1 \\
& \bar{f}_{1}\left(u_{16} u_{1}^{-4} u_{2}^{4} u_{3}^{4}\right)=1 \\
& \bar{f}_{1}\left(u_{17} u_{2}^{-2} u_{4}^{2} u_{6}^{2}\right)=1 \\
& \bar{f}_{1}\left(u_{18} u_{1}^{6} u_{2}^{4} u_{3}^{6} u_{5}^{2} u_{6}^{4} u_{7}^{2}\right)=1 \\
& \bar{f}_{1}\left(u_{19} u_{4}^{-1} u_{8} u_{12}\right)=1 \\
& \bar{f}_{1}\left(u_{20} u_{1}^{-5} u_{4}^{2} u_{5}^{3} u_{9} u_{12}^{2} u_{13}\right)=1 \\
& \bar{f}_{1}\left(u_{21} u_{2} u_{6}^{-3} u_{10} u_{12}^{2} u_{14}\right)=1 \\
& \bar{f}_{1}\left(u_{22} u_{1}^{-3} u_{2}^{3} u_{3}^{3} u_{4}^{2} u_{5}^{7} u_{6} u_{7}^{3} u_{9}^{3} u_{10}^{3} u_{11} u_{13} u_{14} u_{15}\right)=1 \\
& \bar{f}_{1}\left(u_{23} u_{2}^{5} u_{3}^{7} u_{4}^{-1} u_{5}^{5} u_{6}^{2} u_{9}^{2} u_{10} u_{11} u_{12} u_{13}\right)=1 \\
& \bar{f}_{1}\left(u_{24} u_{6}^{5} u_{8} u_{10}^{2} u_{12}^{2} u_{14}\right)=1 \\
& \bar{f}_{1}\left(u_{25} u_{1} u_{2}^{2} u_{3}^{-7} u_{4}^{-1} u_{5}^{2} u_{6}^{-2} u_{7}^{2} u_{9} u_{10}^{2} u_{11} u_{12} u_{14}\right)=1 \\
& \bar{f}_{1}\left(u_{26} u_{1}^{-1} u_{2}^{2} u_{4}^{2} u_{6}^{3} u_{9} u_{10}^{2} u_{14}\right)=1
\end{aligned}
$$

Assim $\operatorname{Im}\left(\bar{f}_{1}\right)=\left\langle e_{1}\right\rangle \times\left\langle e_{2}\right\rangle \times \cdots \times\left\langle e_{15}\right\rangle$ e $\left|\operatorname{Im}\left(\bar{f}_{1}\right)\right|=2^{26}$. Usando o mesmo raciocínio do exemplo 4.3, concluímos

$\operatorname{ker}\left(\bar{f}_{1}\right)=\left\langle\left\{u_{1}^{16}, u_{2}^{8}, u_{3}^{8} u_{1}^{8}, u_{4}^{4}, u_{5}^{4} u_{1}^{-4}, u_{6}^{4} u_{2}^{4}, u_{7}^{4} u_{1}^{-4} u_{2}^{-4} u_{3}^{-4} u_{5}^{4} u_{6}, u_{8}^{2}, u_{9}^{2} u_{1}^{-2}, u_{10}^{2} u_{2}^{6}, u_{11}^{2} u_{1}^{6} u_{2}^{-2} u_{3}^{6} u_{9}^{2} u_{10}^{2}\right.\right.$,

$u_{12}^{2} u_{4}^{2}, u_{13}^{2} u_{1}^{6} u_{4}^{2} u_{5}^{6} u_{9}^{2} u_{12}^{2}, u_{14}^{2} u_{2}^{-2} u_{4}^{2} u_{6}^{-2} u_{10}^{2} u_{12}^{2}, u_{15}^{2} u_{1}^{-6} u_{2}^{2} u_{3}^{6} u_{4}^{2} u_{5}^{-2} u_{6}^{-2} u_{7}^{6} u_{9}^{6} u_{10}^{-2} u_{11}^{2} u_{12}^{-2} u_{13}^{2} u_{14}^{2}$, $u_{16} u_{1}^{-4} u_{2}^{4} u_{3}^{4}, u_{17} u_{2}^{-2} u_{4}^{2} u_{6}^{2}, u_{18} u_{1}^{6} u_{2}^{4} u_{3}^{6} u_{5}^{2} u_{6}^{4} u_{7}^{2}, u_{19} u_{4}^{-1} u_{8} u_{12}, u_{20} u_{1}^{-5} u_{4}^{2} u_{5}^{3} u_{9} u_{12}^{2} u_{13}$ $u_{21} u_{2} u_{6}^{-3} u_{10} u_{12}^{2} u_{14}, u_{22} u_{1}^{-3} u_{2}^{3} u_{3}^{3} u_{4}^{2} u_{5}^{7} u_{6} u_{7}^{3} u_{9}^{3} u_{10}^{3} u_{11} u_{13} u_{14} u_{15}, u_{23} u_{2}^{5} u_{3}^{7} u_{4}^{-1} u_{5}^{5} u_{6}^{2} u_{9}^{2} u_{10} u_{11} u_{12} u_{13}$, $\left.\left.u_{24} u_{6}^{5} u_{8} u_{10}^{2} u_{12}^{2} u_{14}, u_{25} u_{1} u_{2}^{2} u_{3}^{-7} u_{4}^{-1} u_{5}^{2} u_{6}^{-2} u_{7}^{2} u_{9} u_{10}^{2} u_{11} u_{12} u_{14}, u_{26} u_{1}^{-1} u_{2}^{2} u_{4}^{2} u_{6}^{3} u_{9} u_{10}^{2} u_{14}\right\}\right\rangle$

Encontramos deste modo, as bases para ker $\left(\bar{f}_{1}\right)$ e a partir desta base, é possivel encontrar uma base 
para $\operatorname{ker}\left(\bar{\pi}_{1}\right)$, como fizemos em alguns dos exemplos.

\subsection{Validade da hipótese da Proposição 4.2}

Verificaremos agora que a hipótese da Proposição 4.2 é verdadeira para $p^{n}$ tal que $\phi\left(p^{n}\right) \leq 66$, isto é, $(-1)^{p}\left(\mu_{t}\right)^{\frac{\phi\left(p^{n}\right)}{2 p}} \notin \operatorname{Im}\left(\bar{\pi}_{1}\right)$. Mais uma vez a demonstração é feita caso a caso, pois não conseguimos um método geral para provarmos este fato. Logo, caso o número de potências de primos tais que $\left\{ \pm \theta, \mu_{t} \mid 0<t<\frac{p^{n}}{2}, m d c\left(t, p^{n}\right)=1\right\}$ gere $U(\mathbb{Z}[\theta])$ aumente, a prova abaixo não contemplará estas novas potências de primos que vierem a ser encontradas. Fica aqui a dúvida se o resultado valerá de forma mais geral.

Proposição 4.9. Sejam p um primo e $C_{p^{n-1}}=\langle h\rangle$. Considere $U\left(\mathbb{Z}_{p^{n}}\right)=\langle t\rangle$ se p for ímpar e $t=3$ se $p=2$. Então

$$
\varpi=(-1)^{p}\left(1+h+h^{2}+\cdots+h^{t-1}\right)^{\frac{\phi\left(p^{n-1}\right)}{2}}-(-1)^{p} k \cdot \widehat{h} \in U_{1}\left(\mathbb{Z} C_{p^{n-1}}\right),
$$

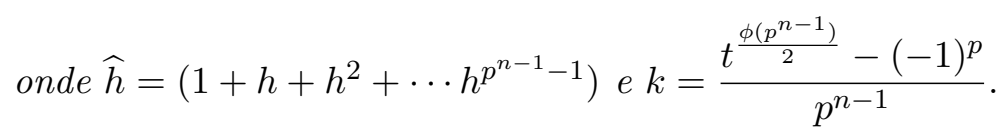

\section{Prova:}

Observe inicialmente que $\varpi$ é um elemento de $\mathbb{Z} C_{p^{n-1}}$. Como $t^{\frac{\phi\left(p^{n-1}\right)}{2}} \equiv(-1)^{p}\left(\bmod p^{n-1}\right)$, então $k=\frac{t^{\frac{\phi\left(p^{n-1}\right)}{2}}-(-1)^{p}}{p^{n-1}} \in \mathbb{Z}$. Logo $\varpi \in \mathbb{Z} C_{p^{n-1}}$.

Agora seja $\omega=(-1)^{p}\left(1+h^{t}+h^{2 t}+\cdots+h^{t(s-1)}\right)^{\frac{\phi\left(p^{n-1}\right)}{2}}-(-1)^{p} k^{\prime} \cdot \widehat{h}$, onde $s$ é o menor inteiro tal que $t s \equiv 1\left(\bmod p^{n-1}\right)$ e $k^{\prime}=\frac{s^{\frac{\phi\left(p^{n-1}\right)}{2}}-(-1)^{p}}{p^{n-1}}$. Provaremos que $\omega=\varpi^{-1}$.

Como $t s \equiv 1\left(\bmod p^{n-1}\right), \quad$ temos $t^{\frac{\phi\left(p^{n-1}\right)}{2}} \cdot s^{\frac{\phi\left(p^{n-1}\right)}{2}} \equiv 1\left(\bmod p^{n-1}\right)$. Como $t^{\frac{\phi\left(p^{n-1}\right)}{2}} \equiv(-1)^{p}\left(\bmod p^{n-1}\right)$, então $s^{\frac{\phi\left(p^{n-1}\right)}{2}} \equiv(-1)^{p}\left(\bmod p^{n-1}\right)$ e $\omega \in \mathbb{Z} C_{p^{n-1}}$.

Agora vamos provar a igualdade $\varpi \cdot \omega=1$.

$$
\begin{aligned}
& \varpi \cdot \omega= \\
& =\left[(-1)^{p}\left(1+h+\cdots+h^{t-1}\right)^{\frac{\phi\left(p^{n-1}\right)}{2}}-(-1)^{p} k \cdot \widehat{h}\right] \cdot\left[(-1)^{p}\left(1+h^{t}+\cdots+h^{t(s-1)}\right)^{\frac{\phi\left(p^{n-1}\right)}{2}}-(-1)^{p} k^{\prime} \cdot \widehat{h}\right]
\end{aligned}
$$


$=\left[(-1)^{p}\left(1+h+\cdots+h^{t-1}\right)^{\frac{\phi\left(p^{n-1}\right)}{2}}\right] \cdot\left[(-1)^{p}\left(1+h^{t}+\cdots+h^{t(s-1)}\right)^{\frac{\phi\left(p^{n-1}\right)}{2}}\right]+R \widehat{h}$

para algum $R \in \mathbb{Z}$

Assim, $\varpi \cdot \omega=\left(1+\frac{s t-1}{p^{n-1}} \widehat{h}\right)^{\frac{\phi\left(p^{n-1}\right)}{2}}+R \widehat{h}=1+R \widehat{h}$, para algum $R_{1} \in \mathbb{Z}$. Como $\varpi$ e $\omega$ tem aumento 1 então $\varpi \cdot \omega=1+R_{1} \widehat{h}$ também tem aumento 1 . Logo $R_{1}=0$ e, então, $\varpi \cdot \omega=1$. ¿

Proposição 4.10. Se $C_{p^{n}}=\langle g\rangle$ e $x=g-1$, então $1+g+g^{2}+\cdots+g^{p^{n}-1}=x^{p^{n}-1}$ em $\mathbb{Z}_{p} C_{p^{n}}$.

\section{Prova:}

Temos os seguintes resultados conhecidos da teoria de números:

1) $p \mid\left(\begin{array}{c}p^{n} \\ k\end{array}\right)$, para $1 \leq k \leq p^{n}-1$;

2) $\left(\begin{array}{c}p^{n}-1 \\ k-1\end{array}\right)+\left(\begin{array}{c}p^{n}-1 \\ k\end{array}\right)=\left(\begin{array}{c}p^{n} \\ k\end{array}\right)$;

Destes fatos podemos concluir que $\left(\begin{array}{c}p^{n}-1 \\ k-1\end{array}\right)+\left(\begin{array}{c}p^{n}-1 \\ k\end{array}\right)=\left(\begin{array}{c}p^{n} \\ k\end{array}\right) \equiv 0(\bmod p)$.

Logo $\left(\begin{array}{c}p^{n}-1 \\ k-1\end{array}\right) \equiv-1\left(\begin{array}{c}p^{n}-1 \\ k\end{array}\right)(\bmod p)$. Como $\left(\begin{array}{c}p^{n}-1 \\ 0\end{array}\right) \equiv 1(\bmod p)$ e $\left(\begin{array}{c}p^{n}-1 \\ 1\end{array}\right) \equiv-1(\bmod p)$, então, para $0 \leq i \leq p^{n}-1$ :

- $\left(\begin{array}{c}p^{n}-1 \\ i\end{array}\right) \equiv 1(\bmod p)$ se $i$ é par

- $\left(\begin{array}{c}p^{n}-1 \\ i\end{array}\right) \equiv-1(\bmod p)$ se $i$ é ímpar

$\operatorname{Assim} x^{p^{n}-1}=(g-1)^{p^{n}-1}=\sum_{i=0}^{p^{n}-1}\left(\begin{array}{c}p^{n}-1 \\ i\end{array}\right)(-1)^{i} g^{i}=1+g+g^{2}+\cdots+g^{p^{n}-1} \cdot \mathbf{z} \mathbf{x}$

Proposição 4.11. Sejam $p$ um primo e $n \in \mathbb{N}, n \geq 2$ tais que $\phi\left(p^{n}\right) \leq 66$, e $\theta$ uma raiz $p^{n}$-ésima primitiva da unidade. Considere $t \in \mathbb{Z}$ tal que $U\left(\mathbb{Z}_{p^{n}}\right)=\langle t\rangle$ se pé ímpar e $t=3$ se $p=2$, e $\mu_{t}=1+\theta+\theta^{2}+\cdots+\theta^{t-1}$ uma unidade ciclotômica de $\mathbb{Z}[\theta]$. Então $(-1)^{p}\left(\mu_{t}\right)^{\frac{\phi\left(p^{n}\right)}{2 p}} \notin \operatorname{Im}\left(\bar{\pi}_{1}\right)$.

\section{Prova:}

Suponha por absurdo que $v=(-1)^{p}\left(\mu_{t}\right)^{\frac{\phi\left(p^{n}\right)}{2 p}}=(-1)^{p}\left(\mu_{t}\right)^{\frac{\phi\left(p^{n-1}\right)}{2}} \in \operatorname{Im}\left(\bar{\pi}_{1}\right)$. Se isto ocorre, existe um elemento $u$ em $U_{1}\left(\mathbb{Z} C_{p^{n}}\right)$ tal que $\bar{\pi}_{1}(u)=(-1)^{p}\left(\mu_{t}\right)^{\frac{\phi\left(p^{n-1}\right)}{2}}=v$. O diagrama abaixo é comutativo e, portanto, $f_{2}(v)=f_{2}\left(\bar{\pi}_{1}(u)\right)=\bar{f}_{1}\left(\pi_{2}(u)\right) \in \operatorname{Im}\left(f_{2}\right) \cap \operatorname{Im}\left(\bar{f}_{1}\right)$.

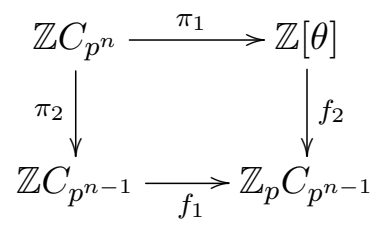


onde $f_{1}$ é o homomorfismo natural, $f_{2}(\theta)=h$, com $C_{p^{n-1}}=\langle h\rangle$ e $\bar{f}_{1}=\left.f_{1}\right|_{U_{1}\left(\mathbb{Z} C_{p^{n-1}}\right)}$.

Como $\varpi=(-1)^{p}\left(1+h+h^{2}+\cdots+h^{t-1}\right)^{\frac{\phi\left(p^{n-1}\right)}{2}}-(-1)^{p} k \cdot \widehat{h} \in U_{1}\left(\mathbb{Z} C_{p^{n-1}}\right)$, onde $k=\frac{t^{\frac{\phi\left(p^{n-1}\right)}{2}}-(-1)^{p}}{p^{n-1}}, \operatorname{assim}$

$$
\bar{f}_{1}(\varpi)=(\overline{-1})^{p}\left(\overline{1}+h+h^{2}+\cdots+h^{t-1}\right)^{\frac{\phi\left(p^{n-1}\right)}{2}}-(\overline{-1})^{p} \bar{k} \cdot \widehat{h}=f_{2}(v)-(\overline{-1})^{p} \bar{k} \cdot \widehat{h} .
$$

Temos ainda $f_{2}(v) \cdot(\bar{k} \cdot \widehat{h})=(\overline{-1})^{p} \frac{\phi\left(p^{n-1}\right)}{2} \cdot \bar{k} \cdot \widehat{h}=\bar{k} \cdot \widehat{h}$, pois $f_{2}(v) \cdot \widehat{h}=(\overline{-1})^{p} \bar{t}^{\frac{\phi\left(p^{n-1}\right)}{2}} \cdot \widehat{h}$ e $(-1)^{p} t^{\frac{\phi\left(p^{n-1}\right)}{2}} \equiv 1\left(\bmod p^{n-1}\right) . \log \mathrm{O}$

$$
\bar{f}_{1}(\varpi)=f_{2}(v)-(\overline{-1})^{p} \bar{k} \cdot \widehat{h}=f_{2}(v) \cdot\left[\overline{1}-(\overline{-1})^{p} \bar{k} \cdot \widehat{h}\right]
$$

Como $f_{2}(v)=\bar{f}_{1}\left(\pi_{2}(u)\right) \in \operatorname{Im}\left(\bar{f}_{1}\right)$ então devemos ter $\left(\overline{1}-(\overline{-1})^{p} \bar{k} \cdot \widehat{h}\right) \in \operatorname{Im}\left(\bar{f}_{1}\right)$.

Fazendo $x=h-1$ e, usando a Proposição 4.10, temos

$$
\overline{1}-(\overline{-1})^{p} \bar{k} \cdot \widehat{h}=\overline{1}-(\overline{-1})^{p} \bar{k} \cdot\left(1+h+\cdots+h^{p^{n-1}-1}\right)=\overline{1}-(\overline{-1})^{p} \bar{k} \cdot x^{p^{n-1}-1} \in \operatorname{Im}\left(\bar{f}_{1}\right) .
$$

Na seção anterior encontramos $\operatorname{Im}\left(\bar{f}_{1}\right)=\left\langle e_{1}\right\rangle \times \cdots\left\langle e_{l}\right\rangle$ em cada um dos casos e facilmente verifica-se que $\overline{1}-(\overline{-1})^{p} \bar{k} \cdot x^{p^{n-1}-1}$ é multiplicativamente independente com $\left\{e_{1}, e_{2}, \cdots, e_{l}\right\}$. O que é um absurdo, portanto, não podemos ter $v=(-1)^{p}\left(\mu_{t}\right)^{\frac{\phi\left(p^{n}\right)}{2 p}} \in \operatorname{Im}\left(\bar{\pi}_{1}\right)$. ¿̇

Na verdade, nas seções anteriores analisamos os casos: $2^{4}, 2^{5}, 2^{6}, 2^{7}, 3^{2}, 3^{3}, 3^{4}, 5^{2}$ e $7^{2}$. Não fizemos os casos $2^{2}$ e $2^{3}$, pois já são conhecidos. O primeiro é trivial e as unidades de $\mathbb{Z} C_{8}$ já foram descritas e uma demonstração pode ser encontrada em [39] na página 251.

Neste trabalho, ficam estabelecidas as bases de $U_{1}\left(\mathbb{Z} C_{p^{n}}\right)$, para $p^{n}$ tal que $\phi\left(p^{n}\right) \leq 66$.

Acreditamos que existe uma forma de provar que $(-1)^{p}\left(\mu_{t}\right)^{\frac{\phi\left(p^{n}\right)}{2 p}} \notin \operatorname{Im}\left(\bar{\pi}_{1}\right)$, mas no momento estes são os resultados que obtivemos. 


\section{Considerações finais}

O objetivo desta tese foi encontrar um conjunto de geradores multiplicativamente independentes para o grupo $U\left(\mathbb{Z} C_{p^{n}}\right)$, para os primos $p$ tais que $\phi\left(p^{n}\right) \leq 66$. Levando-se em conta o que foi observado no desenvolvimento da tese, decompomos o grupo das unidades normalizadas de $\mathbb{Z} C_{p^{n}}$ do seguinte modo:

$$
U_{1}\left(\mathbb{Z} C_{p^{n}}\right)=\operatorname{ker}\left(\bar{\pi}_{1}\right) \times\langle S\rangle,
$$

onde $S \simeq \operatorname{Im}\left(\bar{\pi}_{1}\right)$ e $\bar{\pi}_{1}: U_{1}\left(\mathbb{Z} C_{p^{n}}\right) \longrightarrow U_{1}(\mathbb{Z}[\theta])$ é um homomorfismo de grupos.

Assim, o problema se restringiu em encontrar $S \simeq \operatorname{Im}\left(\bar{\pi}_{1}\right)$ e um conjunto de geradores independentes de $\operatorname{ker}\left(\bar{\pi}_{1}\right)$. Descrevemos $S$ como sendo o conjunto formado pelas unidades de Hoechsmann

$\vartheta_{i}=\left(1+g^{t}+g^{2 t}+\cdots+g^{(r-1) t}\right)\left(1+g^{t^{i}}+g^{2 t^{i}}+\cdots+g^{(t-1) t^{i}}\right)-\frac{(t r-1)}{p^{n}}\left(1+g+g^{2}+\cdots+g^{p^{n}-1}\right) \in U_{1}\left(\mathbb{Z} C_{p^{n}}\right)$,

para $i=1,2, \cdots, \kappa=\frac{\phi\left(p^{n}\right)}{2}-1$.

A dificuldade está em encontrar um conjunto de geradores independentes de $\operatorname{ker}\left(\bar{\pi}_{1}\right)$, de modo mais geral. Exibimos tal conjunto, caso a caso, para cada potência de primo em questão.

Um problema em vista nesta linha de pesquisa é encontrar um conjunto de geradores multiplicativamente independentes para o grupo das unidades do anel de grupo $\mathbb{Z}\left(C_{p} \times C_{p}\right)$. 


\section{Apêndice}

Esta parte se reserva aos detalhes e comandos dos cálculos das seções 4.2 e 4.3.

Os programas utilizados foram MAPLE e GAP. Este último foi usado somente com o intuito de encontrar as unidades simétricas de $\mathbb{Z} C_{p^{n}}$, que foram coladas do GAP e depois copiadas no MAPLE.

No MAPLE encontramos os conjuntos independentes que geram $\operatorname{Im}\left(\bar{f}_{1}\right)$ e também verificamos que $\overline{1}-(\overline{-1})^{p} \bar{k} \cdot x^{p^{n-1}-1}$ não pertence a $\operatorname{Im}\left(\bar{f}_{1}\right)$.

As páginas a seguir são os anexos dos cálculos. 
Aqui estão os cálculos para encontrar um conjunto multiplicativamente independente que gera $\operatorname{Im}\left(f_{1}\right)$. Para não sobrecarregar a notação, $f_{1}$ já está restrito às unidades normalizadas de $\mathbb{Z} C_{p^{n-1}}$.

$u_{i}$ é unidade simétrica normalizada de $\mathbb{Z} C_{p^{n-1}}$ que foi encontrado no programa GAP.

$U_{1}\left(\mathbb{Z}_{p^{n-1}}\right)=\left\langle h, u_{1}, u_{2}, \ldots, u_{k}\right\rangle$

$v_{i}$ é a imagem de $u_{i}$ aplicado ao homomorfismo $f_{1}$, mas já substituindo $h$ por $x+1$. Não confundir o homomorfismo $f_{1}$ com os elementos $f 1, f 2, \ldots f m$ em $u_{i}$ que o programa GAP impôs.

Caso $p=3^{3}$ :

$u_{i}$ é a unidade simétrica de $\mathbb{Z} C_{9}$.

$$
\begin{aligned}
& u_{1}:=\operatorname{sort}\left(\operatorname { s u b s } \left(f 1=h, f 2=h^{3},-1+(1) * f 1+(1) * f 2+(-1) * f l^{\wedge} 2+(1) * f 2^{\wedge} 2+(-1) * f 1^{*} f 2^{\wedge} 2\right.\right. \\
& \left.\left.+(1) * f 1^{\wedge} 2 * f 2^{\wedge} 2\right)\right) \\
& h^{8}-h^{7}+h^{6}+h^{3}-h^{2}+h-1 \\
& v_{1}:=\operatorname{sort}\left(\operatorname{expand}\left(\left(\operatorname{subs}\left(h=x+1, u_{1}\right)\right)\right)\right) \bmod 3 \\
& x^{8}+x^{7}+x^{6}+2 x^{5}+2 x^{4}+1 \\
& u_{2}:=\operatorname{sort}\left(\operatorname{subs}\left(f 1=h, f 2=h^{3},(1)+(-1) * f 1+(1) * f 1^{\wedge} 2+(1) * f 1 * f 2^{\wedge} 2+(-1) * f l^{\wedge} 2 * f 2^{\wedge} 2\right)\right) \\
& -h^{8}+h^{7}+h^{2}-h+1 \\
& v_{2}:=\operatorname{sort}\left(\operatorname{expand}\left(\left(\operatorname{subs}\left(h=x+1, u_{2}\right)\right)\right)\right) \bmod 3 \\
& 2 x^{8}+2 x^{7}+x^{5}+x^{4}+1
\end{aligned}
$$

Vamos encontrar um conjunto multiplicativamente independente que gera $\operatorname{Im}\left(f_{1}\right)=\left\langle h, v_{1}, v_{2}\right\rangle$ $e_{1}:=v_{1}$

$$
x^{8}+x^{7}+x^{6}+2 x^{5}+2 x^{4}+1
$$

$e_{2}:=\operatorname{mtaylor}\left(\operatorname{sort}\left(\operatorname{expand}\left(v_{2} \cdot e_{1}\right)\right), x, 9\right) \bmod 3$

$$
1+x^{6}+2 x^{8}
$$

$\operatorname{Logo} \operatorname{Im}\left(f_{1}\right)=\left\langle h, v_{1}, v_{2}\right\rangle=\langle h\rangle \times\left\langle e_{1}\right\rangle \times\left\langle e_{2}\right\rangle$

$\operatorname{Caso} p=3^{4}:$

$u_{i}$ é a unidade simétrica de $\mathbb{Z} C_{27}$.

$$
\begin{aligned}
u_{1}: & =\operatorname{sort}\left(\operatorname { s u b s } \left(f 1=h, f 2=h^{3}, f 3=h^{9},(1)+(-1) * f 1+(-1) * f 2+(-1) * f 3+(1) * f l^{\wedge} 2+(1)\right.\right. \\
& * f 1 * f 2+(1) * f 1 * f 3+(1) * f 2^{\wedge} 2+(1) * f 2 * f 3+(-1) * f 3^{\wedge} 2+(-1) * f 1^{\wedge} 2 * f 2+(-1) * f 1 \\
& \wedge 2 * f 3+(-1) * f 1 * f 2^{\wedge} 2+(1) * f 1 * f 3^{\wedge} 2+(1) * f 2^{\wedge} 2 * f 3+(1) * f 2^{*} f 3^{\wedge} 2+(1) * f 1^{\wedge} 2 * f 2^{\wedge} 2
\end{aligned}
$$


$+(-1) * f 1^{\wedge} 2 * f 3^{\wedge} 2+(-1) * f 1^{*} f 2^{\wedge} 2 * f 3+(-1) * f l^{*} f 2^{*} f 3^{\wedge} 2+(-1) * f 2^{\wedge} 2 * f 3^{\wedge} 2+(1)$

$\left.\left.* f 1^{\wedge} 2 * f 2^{\wedge} 2 * f 3+(1) * f l^{\wedge} 2 * f 2 * f 3^{\wedge} 2+(1) * f l^{*} f 2^{\wedge} 2 * f 3^{\wedge} 2+(-1) * f l^{\wedge} 2 * f 2^{\wedge} 2 * f 3^{\wedge} 2\right)\right)$ $-h^{26}+h^{25}-h^{24}+h^{23}-h^{22}+h^{21}-h^{20}+h^{19}-h^{18}+h^{17}-h^{16}+h^{15}+h^{12}-h^{11}+h^{10}-h^{9}$

$+h^{8}-h^{7}+h^{6}-h^{5}+h^{4}-h^{3}+h^{2}-h+1$

$v_{1}:=\operatorname{sort}\left(\operatorname{expand}\left(\left(\operatorname{subs}\left(h=x+1, u_{1}\right)\right)\right)\right) \bmod 3$

$1+2 x^{4}+2 x^{5}+x^{6}+x^{7}+x^{8}+2 x^{9}+x^{10}+x^{11}+x^{12}+2 x^{15}+2 x^{16}+2 x^{17}+2 x^{18}+2 x^{19}$

$+2 x^{20}+2 x^{21}+2 x^{22}+2 x^{23}+2 x^{24}+2 x^{25}+2 x^{26}$

$u_{2}:=\operatorname{sort}\left(\operatorname{subs}\left(f l=h, f 2=h^{3}, f 3=h^{9},(-1)+(1) * f 1+(1) * f 2+(1) * f 3+(-1) * f l^{\wedge} 2+(-1)\right.\right.$

$* f 1 * f 2+(-1) * f 1^{*} f 3+(-1) * f 2^{\wedge} 2+(1) * f 3^{\wedge} 2+(1) * f l^{\wedge} 2 * f 2+(1) * f l^{\wedge} 2 * f 3+(1) * f 1$

$* f 2^{\wedge} 2+(-1) * f l^{*} f 3^{\wedge} 2+(-1) * f 2^{*} f 3^{\wedge} 2+(-1) * f l^{\wedge} 2 * f 2^{\wedge} 2+(1) * f l^{\wedge} 2 * f 3^{\wedge} 2+(1) * f 1$

$* f 2^{\wedge} 2 * f 3+(1) * f 1^{*} f 2^{*} f 3^{\wedge} 2+(1) * f 2^{\wedge} 2 * f 3^{\wedge} 2+(-1) * f 1^{\wedge} 2 * f 2^{\wedge} 2 * f 3+(-1) * f 1^{\wedge} 2 * f 2$

$\left.\left.* f 3^{\wedge} 2+(-1) * f 1^{*} f 2^{\wedge} 2 * f 3^{\wedge} 2+(1) * f 1^{\wedge} 2 * f 2^{\wedge} 2 * f 3^{\wedge} 2\right)\right)$

$h^{26}-h^{25}+h^{24}-h^{23}+h^{22}-h^{21}+h^{20}-h^{19}+h^{18}-h^{17}+h^{16}+h^{11}-h^{10}+h^{9}-h^{8}+h^{7}-h^{6}$

$+h^{5}-h^{4}+h^{3}-h^{2}+h-1$

$v_{2}:=\operatorname{sort}\left(\operatorname{expand}\left(\left(\operatorname{subs}\left(h=x+1, u_{2}\right)\right)\right)\right) \bmod 3$

$1+x^{4}+x^{5}+2 x^{7}+2 x^{8}+2 x^{10}+2 x^{11}+2 x^{12}+2 x^{15}+x^{16}+x^{17}+x^{18}+x^{19}+x^{20}+x^{21}+x^{22}$

$+x^{23}+x^{24}+x^{25}+x^{26}$

$u_{3}:=\operatorname{sort}\left(\operatorname{subs}\left(f l=h, f 2=h^{3}, f 3=h^{9},(-1)+(1) * f 1+(1) * f 2+(1) * f 3+(-1) * f l^{\wedge} 2+(-1)\right.\right.$

$* f 1 * f 2+(-1) * f 2^{\wedge} 2+(1) * f 3^{\wedge} 2+(1) * f 1^{\wedge} 2 * f 2+(1) * f 1^{*} f 2^{\wedge} 2+(-1) * f 1^{*} f 3^{\wedge} 2+(-1)$

$* f 2^{*} f 3^{\wedge} 2+(-1)^{*} f l^{\wedge} 2 * f 2^{\wedge} 2+(1) * f 1^{\wedge} 2 * f 3^{\wedge} 2+(1) * f l^{*} f 2^{*} f 3^{\wedge} 2+(1) * f 2^{\wedge} 2 * f 3^{\wedge} 2+($

$\left.\left.-1) * f l^{\wedge} 2 * f 2 * f 3^{\wedge} 2+(-1) * f 1^{*} f 2^{\wedge} 2 * f 3^{\wedge} 2+(1) * f l^{\wedge} 2 * f 2^{\wedge} 2 * f 3^{\wedge} 2\right)\right)$

$h^{26}-h^{25}+h^{24}-h^{23}+h^{22}-h^{21}+h^{20}-h^{19}+h^{18}+h^{9}-h^{8}+h^{7}-h^{6}+h^{5}-h^{4}+h^{3}-h^{2}+h$

$-1$

$v_{3}:=\operatorname{sort}\left(\operatorname{expand}\left(\left(\operatorname{subs}\left(h=x+1, u_{3}\right)\right)\right)\right) \bmod 3$

$1+2 x^{10}+2 x^{11}+2 x^{12}+2 x^{13}+2 x^{14}+2 x^{15}+2 x^{16}+2 x^{17}+x^{18}+x^{19}+x^{20}+x^{21}+x^{22}+x^{23}$

$+x^{24}+x^{25}+x^{26}$

$u_{4}:=\operatorname{sort}\left(\operatorname{subs}\left(f l=h, f 2=h^{3}, f 3=h^{9},(-1)+(1) * f 1+(1) * f 2+(-1) * f I^{\wedge} 2+(-1) * f 1 * f 2\right.\right.$

$+(1) * f l^{\wedge} 2 * f 2+(1) * f 1 * f 2^{*} f 3^{\wedge} 2+(1) * f 2^{\wedge} 2 * f 3^{\wedge} 2+(-1) * f l^{\wedge} 2 * f 2 * f 3^{\wedge} 2+(-1) * f 1$

$\left.\left.* f 2^{\wedge} 2 * f 3^{\wedge} 2+(1) * f 1^{\wedge} 2 * f 2^{\wedge} 2 * f 3^{\wedge} 2\right)\right)$

$$
h^{26}-h^{25}+h^{24}-h^{23}+h^{22}+h^{5}-h^{4}+h^{3}-h^{2}+h-1
$$

$v_{4}:=\operatorname{sort}\left(\operatorname{expand}\left(\left(\operatorname{subs}\left(h=x+1, u_{4}\right)\right)\right)\right) \bmod 3$

$1+2 x^{4}+2 x^{5}+x^{6}+x^{7}+x^{8}+2 x^{9}+x^{12}+2 x^{13}+2 x^{14}+2 x^{15}+2 x^{16}+2 x^{17}+x^{18}+2 x^{21}$

$+x^{22}+x^{23}+x^{24}+x^{25}+x^{26}$

$u_{5}:=\operatorname{sort}\left(\operatorname{subs}\left(f 1=h, f 2=h^{3}, f 3=h^{9},(1)+(-1) * f 1+(1) * f l^{\wedge} 2+(1) * f I^{*} f 2^{\wedge} 2 * f 3^{\wedge} 2+(-1)\right.\right.$

$\left.\left.* f 1^{\wedge} 2 * f 2^{\wedge} 2 * f 3^{\wedge} 2\right)\right)$

$$
-h^{26}+h^{25}+h^{2}-h+1
$$

$v_{5}:=\operatorname{sort}\left(\operatorname{expand}\left(\left(\operatorname{subs}\left(h=x+1, u_{5}\right)\right)\right)\right) \bmod 3$

$2 x^{26}+2 x^{25}+x^{23}+x^{22}+2 x^{20}+2 x^{19}+x^{17}+x^{16}+2 x^{14}+2 x^{13}+x^{11}+x^{10}+2 x^{8}+2 x^{7}+x^{5}$

$+x^{4}+1$

$u_{6}:=\operatorname{sort}\left(\operatorname{subs}\left(f l=h, f 2=h^{3}, f 3=h^{9},(1)+(-1) * f l+(-1) * f 2+(1) * f l^{\wedge} 2+(1) * f 1 * f 2+(1)\right.\right.$

$* f 2^{\wedge} 2+(-1) * f 1^{\wedge} 2 * f 2+(-1) * f 1 * f 2^{\wedge} 2+(1) * f 1^{*} f 3^{\wedge} 2+(1) * f 2 * f 3^{\wedge} 2+(1) * f 1^{\wedge} 2 * f 2^{\wedge} 2$ 
$+(-1) * f 1^{\wedge} 2 * f 3^{\wedge} 2+(-1) * f 1^{*} f 2^{*} f 3^{\wedge} 2+(-1) * f 2^{\wedge} 2 * f 3^{\wedge} 2+(1) * f 1^{\wedge} 2 * f 2^{*} f 3^{\wedge} 2+(1)$

$\left.\left.* f 1^{*} f 2^{\wedge} 2 * f 3^{\wedge} 2+(-1) * f 1^{\wedge} 2 * f 2^{\wedge} 2 * f 3^{\wedge} 2\right)\right)$

$-h^{26}+h^{25}-h^{24}+h^{23}-h^{22}+h^{21}-h^{20}+h^{19}+h^{8}-h^{7}+h^{6}-h^{5}+h^{4}-h^{3}+h^{2}-h+1$

(17)

$v_{6}:=\operatorname{sort}\left(\right.$ expand $\left.\left(\left(\operatorname{subs}\left(h=x+1, u_{6}\right)\right)\right)\right) \bmod 3$

$2 x^{26}+2 x^{25}+2 x^{24}+2 x^{23}+2 x^{22}+2 x^{21}+2 x^{20}+2 x^{19}+x^{17}+x^{16}+x^{15}+x^{14}+x^{13}+x^{12}$

$+x^{11}+x^{10}+1$

$u_{7}:=\operatorname{sort}\left(\operatorname{subs}\left(f 1=h, f 2=h^{3}, f 3=h^{9},(-1)+(1) * f 1+(1) * f 2+(-1) * f l^{\wedge} 2+(1) * f 2^{\wedge} 2 * f 3^{\wedge} 2\right.\right.$

$\left.\left.+(-1) * f l^{*} f 2^{\wedge} 2 * f 3^{\wedge} 2+(1) * f 1^{\wedge} 2 * f 2^{\wedge} 2 * f 3^{\wedge} 2\right)\right)$

$h^{26}-h^{25}+h^{24}+h^{3}-h^{2}+h-1$

$v_{7}:=\operatorname{sort}\left(\operatorname{expand}\left(\left(\operatorname{subs}\left(h=x+1, u_{7}\right)\right)\right)\right) \bmod 3$

$x^{26}+x^{25}+x^{24}+2 x^{23}+2 x^{22}+2 x^{21}+x^{20}+x^{19}+x^{18}+2 x^{17}+2 x^{16}+2 x^{15}+x^{14}+x^{13}+x^{12}$

$+2 x^{11}+2 x^{10}+2 x^{9}+x^{8}+x^{7}+x^{6}+2 x^{5}+2 x^{4}+1$

$u_{8}:=\operatorname{sort}\left(\operatorname{subs}\left(f l=h, f 2=h^{3}, f 3=h^{9},(1)+(-1) * f 1+(-1) * f 2+(1) * f l^{\wedge} 2+(1) * f 1 * f 2+(1)\right.\right.$

$* f 2^{\wedge} 2+(-1) * f 1^{\wedge} 2 * f 2+(1) * f 2 * f 3^{\wedge} 2+(-1) * f 1 * f 2 * f 3^{\wedge} 2+(-1) * f 2^{\wedge} 2 * f 3^{\wedge} 2+(1) * f 1$

$\left.\left.\wedge 2 * f 2 * f 3^{\wedge} 2+(1) * f 1^{*} f 2^{\wedge} 2 * f 3^{\wedge} 2+(-1) * f 1^{\wedge} 2 * f 2^{\wedge} 2 * f 3^{\wedge} 2\right)\right)$

$$
-h^{26}+h^{25}-h^{24}+h^{23}-h^{22}+h^{21}+h^{6}-h^{5}+h^{4}-h^{3}+h^{2}-h+1
$$

$v_{8}:=\operatorname{sort}\left(\right.$ expand $\left.\left(\left(\operatorname{subs}\left(h=x+1, u_{8}\right)\right)\right)\right) \bmod 3$

$2 x^{26}+2 x^{25}+2 x^{24}+2 x^{23}+2 x^{22}+2 x^{21}+x^{17}+x^{16}+x^{15}+x^{14}+x^{13}+x^{12}+2 x^{8}+2 x^{7}+x^{5}$

$+x^{4}+1$

$u_{9}:=\operatorname{sort}\left(\operatorname{subs}\left(f l=h, f 2=h^{3}, f 3=h^{9},(-11)+(11) * f 1+(6) * f 2+(-12) * f 3+(-9) * f l^{\wedge} 2+(\right.\right.$

$-2) * f 1 * f 2+(11) * f 1 * f 3+(6) * f 2^{\wedge} 2+(6) * f 2 * f 3+(-12) * f 3^{\wedge} 2+(-2) * f 1^{\wedge} 2 * f 2+($

$-9) * f l^{\wedge} 2 * f 3+(-9) * f 1^{*} f 2^{\wedge} 2+(-2) * f 1 * f 2 * f 3+(11) * f l^{*} f 3^{\wedge} 2+(6) * f 2^{\wedge} 2 * f 3+(6)$

$* f 2^{*} f 3^{\wedge} 2+(11)^{*} f 1^{\wedge} 2 * f 2^{\wedge} 2+(-2)^{*} f 1^{\wedge} 2 * f 2^{*} f 3+(-9) * f 1^{\wedge} 2 * f 3^{\wedge} 2+(-9) * f 1^{*} f 2^{\wedge} 2 * f 3$

$+(-2) * f l^{*} f 2^{*} f 3^{\wedge} 2+(6) * f 2^{\wedge} 2 * f 3^{\wedge} 2+(11) * f l^{\wedge} 2 * f 2^{\wedge} 2 * f 3+(-2) * f 1^{\wedge} 2 * f 2^{*} f 3^{\wedge} 2+($

$\left.\left.-9) * f 1^{*} f 2^{\wedge} 2 * f 3^{\wedge} 2+(11) * f 1^{\wedge} 2 * f 2^{\wedge} 2 * f 3^{\wedge} 2\right)\right)$

$11 h^{26}-9 h^{25}+6 h^{24}-2 h^{23}-2 h^{22}+6 h^{21}-9 h^{20}+11 h^{19}-12 h^{18}+11 h^{17}-9 h^{16}+6 h^{15}$

$-2 h^{14}-2 h^{13}+6 h^{12}-9 h^{11}+11 h^{10}-12 h^{9}+11 h^{8}-9 h^{7}+6 h^{6}-2 h^{5}-2 h^{4}+6 h^{3}$

$-9 h^{2}+11 h-11$

$v_{9}:=\operatorname{sort}\left(\operatorname{expand}\left(\left(\operatorname{subs}\left(h=x+1, u_{9}\right)\right)\right)\right) \bmod 3$

$$
2 x^{26}+x^{25}+2 x^{24}+2 x^{23}+2 x^{22}+1
$$

$u_{10}:=\operatorname{sort}\left(\operatorname{subs}\left(f l=h, f 2=h^{3}, f 3=h^{9},(7)+(-6) * f l+(-3) * f 2+(6) * f 3+(5) * f l^{\wedge} 2+(1)\right.\right.$

$* f 1 * f 2+(-6) * f 1 * f 3+(-3) * f 2^{\wedge} 2+(-3) * f 2 * f 3+(6) * f 3^{\wedge} 2+(1) * f 1^{\wedge} 2 * f 2+(5) * f 1$

$\wedge 2 * f 3+(5) * f l^{*} f 2^{\wedge} 2+(1) * f l^{*} f 2^{*} f 3+(-6) * f 1^{*} f 3^{\wedge} 2+(-3) * f 2^{\wedge} 2 * f 3+(-3) * f 2^{*} f 3^{\wedge} 2$

$+(-6) * f l^{\wedge} 2 * f 2^{\wedge} 2+(1) * f l^{\wedge} 2 * f 2 * f 3+(5) * f 1^{\wedge} 2 * f 3 \wedge 2+(5) * f 1^{*} f 2^{\wedge} 2 * f 3+(1) * f 1^{*} f 2$

$* f 3^{\wedge} 2+(-3) * f 2^{\wedge} 2 * f 3^{\wedge} 2+(-6) * f 1^{\wedge} 2 * f 2^{\wedge} 2 * f 3+(1) * f 1^{\wedge} 2 * f 2 * f 3^{\wedge} 2+(5) * f 1 * f 2^{\wedge} 2 * f 3$

$\left.\left.\wedge 2+(-6) * f l^{\wedge} 2 * f 2^{\wedge} 2 * f 3^{\wedge} 2\right)\right)$

$-6 h^{26}+5 h^{25}-3 h^{24}+h^{23}+h^{22}-3 h^{21}+5 h^{20}-6 h^{19}+6 h^{18}-6 h^{17}+5 h^{16}-3 h^{15}+h^{14}$

$+h^{13}-3 h^{12}+5 h^{11}-6 h^{10}+6 h^{9}-6 h^{8}+5 h^{7}-3 h^{6}+h^{5}+h^{4}-3 h^{3}+5 h^{2}-6 h+7$

$v_{10}:=\operatorname{sort}\left(\operatorname{expand}\left(\left(\operatorname{subs}\left(h=x+1, u_{10}\right)\right)\right)\right) \bmod 3$

$$
2 x^{25}+2 x^{24}+x^{23}+x^{22}+1
$$

Assim $\operatorname{Im}\left(f_{1}\right)=\left\langle h, v_{1}, v_{2}, \ldots, v_{10}\right\rangle$. Vamos encontrar um conjunto multiplicativamente independente que gera 


$$
\begin{aligned}
& \operatorname{Im}\left(f_{1}\right): \\
& e_{1}:=\text { mtaylor }\left(\operatorname{sort}\left(\text { expand }\left(v_{1}\right)\right), x, 27\right) \bmod 3 \\
& 2 x^{26}+2 x^{25}+2 x^{24}+2 x^{23}+2 x^{22}+2 x^{21}+2 x^{20}+2 x^{19}+2 x^{18}+2 x^{17}+2 x^{16}+2 x^{15}+x^{12} \\
& +x^{11}+x^{10}+2 x^{9}+x^{8}+x^{7}+x^{6}+2 x^{5}+2 x^{4}+1 \\
& e_{2}:=\operatorname{mtaylor}\left(\operatorname{sort}\left(\operatorname{expand}\left(v_{2} \cdot e_{1}\right)\right), x, 27\right) \bmod 3 \\
& 1+x^{6}+2 x^{8}+x^{12}+2 x^{14}+x^{18}+2 x^{20}+x^{24}+2 x^{26} \\
& e_{3}:=\operatorname{mtaylor}\left(\operatorname{sort}\left(\text { expand }\left(v_{3}\right)\right), x, 27\right) \bmod 3 \\
& x^{26}+x^{25}+x^{24}+x^{23}+x^{22}+x^{21}+x^{20}+x^{19}+x^{18}+2 x^{17}+2 x^{16}+2 x^{15}+2 x^{14}+2 x^{13}+2 x^{12} \\
& +2 x^{11}+2 x^{10}+1 \\
& e_{4}:=\text { mtaylor }\left(\operatorname{sort}\left(\text { expand }\left(v_{4} \cdot e_{1}^{2} \cdot e_{3}^{2}\right)\right), x, 27\right) \bmod 3 \\
& 1+2 x^{14}+x^{15}+x^{16}+2 x^{17}+x^{18}+x^{20}+2 x^{21}+2 x^{23}+2 x^{25} \\
& e_{5}:=\text { mtaylor }\left(\operatorname{sort}\left(\text { expand }\left(v_{5} \cdot e_{1}^{7} \cdot e_{2}^{2} \cdot e_{3}^{2} \cdot e_{4}\right)\right), x, 27\right) \bmod 3 \\
& 1+2 x^{16}+2 x^{17}+x^{18}+x^{25} \\
& e_{6}:=\text { mtaylor }\left(\operatorname{sort}\left(\operatorname{expand}\left(v_{6} \cdot e_{3} \cdot e_{2}^{6}\right)\right), x, 27\right) \bmod 3 \\
& 1+2 x^{20}+x^{21}+2 x^{23}+2 x^{24}+2 x^{26} \\
& e_{7}:=\text { mtaylor }\left(\operatorname{sort}\left(\text { expand }\left(v_{7} \cdot e_{1}^{5} \cdot e_{3} \cdot e_{4} \cdot e_{2}^{6} \cdot e_{6}^{2}\right)\right), x, 27\right) \bmod 3 \\
& 2 x^{26}+x^{25}+2 x^{24}+2 x^{23}+2 x^{22}+1 \\
& e_{8}:=\text { mtaylor }\left(\operatorname{sort}\left(\text { expand }\left(v_{8} \cdot e_{1}^{4} \cdot e_{2}^{2} \cdot e_{3} \cdot e_{4}^{2} \cdot e_{5} \cdot e_{6}^{2} \cdot e_{7}\right)\right), x, 27\right) \bmod 3 \\
& 1+x^{24}+2 x^{26} \\
& e_{9}:=\text { mtaylor }\left(\operatorname{sort}\left(\text { expand }\left(v_{9} \cdot e_{7}^{2}\right)\right), x, 27\right) \bmod 3 \quad \# \text { igual a } v_{1}^{5} v_{3} v_{4}^{2} v_{6} v_{7}^{2} \cdot v_{9} \\
& 1 \\
& e_{10}:=\text { mtaylor }\left(\operatorname{sort}\left(\operatorname{expand}\left(v_{10} \cdot e_{7} \cdot e_{8}^{2}\right)\right), x, 27\right) \bmod 3 \quad \# \text { igual } a v_{1}^{6} v_{2}^{5} v_{3} v_{5}^{2} v_{6} v_{7}^{3} v_{8}^{2} v_{10} \\
& 1
\end{aligned}
$$

Caso $p=5^{2}:$

$u_{1}=-1+h+h^{4}$ é a unidade simétrica normalizada de $\mathbb{Z} C_{5}$.

$$
\begin{array}{r}
v_{1}:=\operatorname{sort}\left(\operatorname{expand}\left(\left(\operatorname{subs}\left(h=x+1,-1+h+h^{4}\right)\right)\right)\right) \bmod 5 \\
x^{4}+4 x^{3}+x^{2}+1
\end{array}
$$

$\operatorname{sort}\left(\operatorname{expand}\left(v_{1}^{5}\right)\right) \bmod 5$

$$
x^{20}+4 x^{15}+x^{10}+1
$$


$v_{1}^{5}=1$ pois $x^{5}=0$ em $\mathbb{Z}_{5} C_{5}$.

Portanto, $\operatorname{Im}\left(f_{1}\right)=\left\langle h, v_{1}\right\rangle$.

\section{Caso $p=7^{2}$ :}

$u_{i}$ é a unidade simétrica normalizada de $\mathbb{Z} C_{7}$

$$
\begin{aligned}
& u_{1}:=-1+h+h^{6} \\
& -1+h+h^{6} \\
& v_{1}:=\operatorname{sort}\left(\operatorname{expand}\left(\left(\operatorname{subs}\left(h=x+1, u_{1}\right)\right)\right)\right) \bmod 7 \\
& x^{6}+6 x^{5}+x^{4}+6 x^{3}+x^{2}+1 \\
& u_{2}:=1-h^{2}+h^{3}+h^{4}-h^{5} \\
& 1-h^{2}+h^{3}+h^{4}-h^{5} \\
& v_{2}:=\operatorname{sort}\left(\operatorname{expand}\left(\left(\operatorname{subs}\left(h=x+1, u_{2}\right)\right)\right)\right) \bmod 7 \\
& 6 x^{5}+3 x^{4}+2 x^{3}+5 x^{2}+1
\end{aligned}
$$

Vamos encontrar um conjunto multiplicativamente independente que gera $\operatorname{Im}\left(f_{1}\right)=\left\langle h, v_{1}, v_{2}\right\rangle$

$$
\begin{aligned}
& e_{1}:=v_{1} \\
& x^{6}+6 x^{5}+x^{4}+6 x^{3}+x^{2}+1 \\
& e_{2}:=\operatorname{sort}\left(\text { mtaylor }\left(\left(\operatorname{expand}\left(v_{2} \cdot e_{1}^{2}\right)\right), x, 7\right)\right) \bmod 7 \\
& 1+2 x^{4}+3 x^{5}+x^{6} \\
& \operatorname{Logo} \operatorname{Im}\left(f_{1}\right)=\left\langle v_{1}, v_{2}\right\rangle=\langle h\rangle \times\left\langle e_{1}\right\rangle \times\left\langle e_{2}\right\rangle
\end{aligned}
$$

\section{Caso $p=2^{5}$ :}

$u_{i}$ é a unidade simétrica normalizada de $\mathbb{Z} C_{16}$.

$$
\begin{aligned}
& u_{1}:=\operatorname{sort}\left(\operatorname { s u b s } \left(f 1=h, f 2=h^{2}, f 3=h^{4}, f 4=h^{8},(1)+(-1) * f 2+(1) * f 3+(-2) * f 4+(1) * f 1 * f 2\right.\right. \\
& +(-2) * f 1 * f 3+(1) * f 1 * f 4+(1) * f 2 * f 3+(1) * f 2 * f 4+(1) * f 3 * f 4+(1) * f 1 * f 2 * f 3+( \\
& -2) * f 1 * f 2 * f 4+(1) * f 1 * f 3 * f 4+(-1) * f 2 * f 3 * f 4)) \\
& -h^{14}+h^{13}+h^{12}-2 h^{11}+h^{10}+h^{9}-2 h^{8}+h^{7}+h^{6}-2 h^{5}+h^{4}+h^{3}-h^{2}+1 \\
& v_{1}:=\operatorname{sort}\left(\operatorname{expand}\left(\left(\operatorname{subs}\left(h=x+1, u_{1}\right)\right)\right)\right) \bmod 2 \\
& x^{14}+x^{13}+x^{12}+x^{8}+x^{7}+x^{6}+1 \\
& u_{2}:=\operatorname{sort}\left(\operatorname { s u b s } \left(f l=h, f 2=h^{2}, f 3=h^{4}, f 4=h^{8},(1)+(-1) * f 2+(1) * f 1 * f 2+(-1) * f 1 * f 3+(1)\right.\right. \\
& * f 2 * f 3+(1) * f 2 * f 4+(-1) * f 1 * f 2 * f 4+(1) * f 1 * f 3 * f 4+(-1) * f 2 * f 3 * f 4)) \\
& -h^{14}+h^{13}-h^{11}+h^{10}+h^{6}-h^{5}+h^{3}-h^{2}+1 \\
& v_{2}:=\operatorname{sort}\left(\operatorname{expand}\left(\left(\operatorname{subs}\left(h=x+1, u_{2}\right)\right)\right)\right) \bmod 2 \\
& x^{14}+x^{13}+x^{11}+x^{10}+1
\end{aligned}
$$




$$
\begin{aligned}
& u_{3}:=\operatorname{sort}\left(\operatorname { s u b s } \left(f 1=h, f 2=h^{2}, f 3=h^{4}, f 4=h^{8},(-1)+(1) * f 1+(1) * f 3+(-1) * f 1 * f 2+(1) * f 3\right.\right. \\
& * f 4+(-1) * f 1 * f 3 * f 4+(1) * f 1 * f 2 * f 3 * f 4)) \\
& h^{15}-h^{13}+h^{12}+h^{4}-h^{3}+h-1 \\
& v_{3}:=\operatorname{sort}\left(\operatorname{expand}\left(\left(\operatorname{subs}\left(h=x+1, u_{3}\right)\right)\right)\right) \bmod 2 \\
& x^{15}+x^{14}+x^{12}+x^{11}+x^{10}+x^{8}+x^{7}+x^{6}+1 \\
& u_{4}:=\operatorname{sort}\left(\operatorname { s u b s } \left(f l=h, f 2=h^{2}, f 3=h^{4}, f 4=h^{8},(5)+(3) * f 1+(-4) * f 3+(4) * f 4+(-3) * f 1 * f 2\right.\right. \\
& +(-3) * f 1 * f 3+(3) * f 1 * f 4+(-4) * f 3 * f 4+(3) * f 1 * f 2 * f 3+(-3) * f 1 * f 2 * f 4+(-3) * f 1 \\
& * f 3 * f 4+(3) * f l * f 2 * f 3 * f 4)) \\
& 3 h^{15}-3 h^{13}-4 h^{12}-3 h^{11}+3 h^{9}+4 h^{8}+3 h^{7}-3 h^{5}-4 h^{4}-3 h^{3}+3 h+5 \\
& v_{4}:=\operatorname{sort}\left(\operatorname{expand}\left(\left(\operatorname{subs}\left(h=x+1, u_{4}\right)\right)\right)\right) \bmod 2 \\
& x^{15}+x^{14}+1
\end{aligned}
$$

Vamos encontrar um conjunto multiplicativamente independente que gera $\operatorname{Im}\left(f_{1}\right)=\left\langle h, v_{1}, v_{2}, v_{3}, v_{4}\right\rangle$

$$
\begin{aligned}
& e_{1}:=v_{1} \\
& e_{2}:=v_{2} \quad \begin{array}{r}
x^{14}+x^{13}+x^{12}+x^{8}+x^{7}+x^{6}+1 \\
x^{14}+x^{13}+x^{11}+x^{10}+1
\end{array} \\
& \left.e_{3}:=\operatorname{sort}\left(\text { mtaylor (expand }\left(v_{3} \cdot e_{1}^{3} \cdot e_{2}\right), x, 16\right)\right) \bmod 2 \# \text { igual } v_{4} \\
& x^{15}+x^{14}+1
\end{aligned}
$$

sort $\left(\right.$ mtaylor $\left.\left(\operatorname{expand}\left(e_{3} \cdot v_{4}\right), x, 16\right)\right) \bmod 2$

Logo $\operatorname{Im}\left(f_{1}\right)=\left\langle h, v_{1}, v_{2}\right\rangle=\langle h\rangle \times\left\langle e_{1}\right\rangle \times\left\langle e_{2}\right\rangle \times\left\langle e_{3}\right\rangle$

\section{Caso $2^{6}$ :}

$u_{i}$ é a unidade simétrica de $\mathbb{Z} C_{32}$

$$
\begin{aligned}
& u_{1}:=\operatorname{sort}\left(\operatorname { s u b s } \left(f 1=h, f 2=h^{2}, f 3=h^{4}, f 4=h^{8}, f 5=h^{16},-1+(1) * f 1+(2) * f 3+(-1) * f 4+(2) * f 5\right.\right. \\
& +(-1) * f 1 * f 2+(-1) * f 1 * f 3+(-1) * f 1 * f 4+(-1) * f 1 * f 5+(-1) * f 2 * f 3+(2) * f 2 * f 4 \\
& +(-1) * f 2 * f 5+(-1) * f 3 * f 4+(-1) * f 3 * f 5+(-1) * f 4 * f 5+(2) * f 1 * f 2 * f 3+(-1) * f 1 \\
& * f 2 * f 4+(2) * f 1 * f 2 * f 5+(2) * f 1 * f 3 * f 4+(-1) * f 1 * f 3 * f 5+(2) * f 1 * f 4 * f 5+(-1) * f 2 \\
& * f 3 * f 4+(2) * f 2 * f 3 * f 5+(-1) * f 2 * f 4 * f 5+(2) * f 3 * f 4 * f 5+(-1) * f 1 * f 2 * f 3 * f 4+(-1) \\
& * f 1 * f 2 * f 3 * f 5+(-1) * f 1 * f 2 * f 4 * f 5+(-1) * f 1 * f 3 * f 4 * f 5+(1) * f 1 * f 2 * f 3 * f 4 * f 5)) \\
& h^{31}-h^{29}+2 h^{28}-h^{27}-h^{26}+2 h^{25}-h^{24}-h^{23}+2 h^{22}-h^{21}-h^{20}+2 h^{19}-h^{18} \\
& -h^{17}+2 h^{16}-h^{15}-h^{14}+2 h^{13}-h^{12}-h^{11}+2 h^{10}-h^{9}-h^{8}+2 h^{7}-h^{6}-h^{5} \\
& +2 h^{4}-h^{3}+h-1 \\
& u_{2}:=\operatorname{sort}\left(\operatorname { s u b s } \left(f l=h, f 2=h^{2}, f 3=h^{4}, f 4=h^{8}, f 5=h^{16},(-1)+(1) * f 1+(1) * f 3+(2) * f 5+(-1)\right.\right. \\
& * f 1 * f 2+(-1) * f 1 * f 4+(-1) * f 1 * f 5+(-1) * f 2 * f 3+(2) * f 2 * f 4+(-1) * f 2 * f 5+(-1)
\end{aligned}
$$


$* f 3 * f 4+(-1) * f 3 * f 5+(1) * f 1 * f 2 * f 3+(-1) * f 1 * f 2 * f 4+(2) * f 1 * f 2 * f 5+(2) * f 1 * f 3$

$* f 4+(-1) * f 1 * f 3 * f 5+(1) * f 1 * f 4 * f 5+(-1) * f 2 * f 3 * f 4+(2) * f 2 * f 3 * f 5+(-1) * f 2 * f 4$

$* f 5+(1) * f 3 * f 4 * f 5+(-1) * f 1 * f 2 * f 3 * f 4+(-1) * f 1 * f 2 * f 3 * f 5+(-1) * f 1 * f 3 * f 4 * f 5$

$+(1) * f 1 * f 2 * f 3 * f 4 * f 5))$

$h^{31}-h^{29}+h^{28}-h^{26}+h^{25}-h^{23}+2 h^{22}-h^{21}-h^{20}+2 h^{19}-h^{18}-h^{17}+2 h^{16}$

(58)

$-h^{15}-h^{14}+2 h^{13}-h^{12}-h^{11}+2 h^{10}-h^{9}+h^{7}-h^{6}+h^{4}-h^{3}+h-1$

$u_{3}:=\operatorname{sort}\left(\operatorname{subs}\left(f l=h, f 2=h^{2}, f 3=h^{4}, f 4=h^{8}, f 5=h^{16},(-1)+(1) * f 1+(1) * f 3+(-1) * f l * f 2\right.\right.$

$+(1) * f 3 * f 4 * f 5+(-1) * f 1 * f 3 * f 4 * f 5+(1) * f 1 * f 2 * f 3 * f 4 * f 5))$

$$
h^{31}-h^{29}+h^{28}+h^{4}-h^{3}+h-1
$$

$u_{4}:=\operatorname{sort}\left(\operatorname{subs}\left(f l=h, f 2=h^{2}, f 3=h^{4}, f 4=h^{8}, f 5=h^{16},(-1)+(1) * f 1+(1) * f 3+(2) * f 5+(-1)\right.\right.$

$* f 1 * f 2+(-1) * f 1 * f 4+(-1) * f 1 * f 5+(-1) * f 2 * f 3+(1) * f 2 * f 4+(-1) * f 3 * f 4+(-1)$

$* f 3 * f 5+(1) * f 1 * f 2 * f 3+(1) * f 1 * f 2 * f 5+(1) * f 1 * f 3 * f 4+(1) * f 1 * f 4 * f 5+(1) * f 2 * f 3$

$* f 5+(-1) * f 2 * f 4 * f 5+(1) * f 3 * f 4 * f 5+(-1) * f 1 * f 2 * f 3 * f 4+(-1) * f 1 * f 2 * f 3 * f 5+(-1)$

$* f 1 * f 3 * f 4 * f 5+(1) * f 1 * f 2 * f 3 * f 4 * f 5))$

$h^{31}-h^{29}+h^{28}-h^{26}+h^{25}-h^{23}+h^{22}-h^{20}+h^{19}-h^{17}+2 h^{16}-h^{15}+h^{13}-h^{12}$

(60)

$+h^{10}-h^{9}+h^{7}-h^{6}+h^{4}-h^{3}+h-1$

$u_{5}:=\operatorname{sort}\left(\operatorname{subs}\left(f 1=h, f 2=h^{2}, f 3=h^{4}, f 4=h^{8}, f 5=h^{16},(1)+(-1) * f 2+(-1) * f 4+(1) * f 1 * f 2+(\right.\right.$

$-1) * f 1 * f 3+(1) * f 1 * f 4+(1) * f 2 * f 3+(1) * f 3 * f 4+(1) * f 3 * f 5+(-1) * f 4 * f 5+(-1) * f 1$

$* f 2 * f 4+(-1) * f 1 * f 3 * f 5+(1) * f 2 * f 4 * f 5+(1) * f 1 * f 2 * f 3 * f 5+(-1) * f 1 * f 2 * f 4 * f 5$

$+(1) * f 1 * f 3 * f 4 * f 5+(-1) * f 2 * f 3 * f 4 * f 5))$

$-h^{30}+h^{29}-h^{27}+h^{26}-h^{24}+h^{23}-h^{21}+h^{20}+h^{12}-h^{11}+h^{9}-h^{8}+h^{6}-h^{5}+h^{3}$

(61)

$-h^{2}+1$

$u_{6}:=\operatorname{sort}\left(\operatorname{subs}\left(f 1=h, f 2=h^{2}, f 3=h^{4}, f 4=h^{8}, f 5=h^{16},(1)+(-1) * f 2+(1) * f 1 * f 2+(-1) * f 1 * f 3\right.\right.$

$+(1) * f 2 * f 3+(1) * f 2 * f 4 * f 5+(-1) * f 1 * f 2 * f 4 * f 5+(1) * f 1 * f 3 * f 4 * f 5+(-1) * f 2 * f 3$

$* f 4 * f 5))$

$$
-h^{30}+h^{29}-h^{27}+h^{26}+h^{6}-h^{5}+h^{3}-h^{2}+1
$$

$u_{7}:=\operatorname{sort}\left(\operatorname{subs}\left(f 1=h, f 2=h^{2}, f 3=h^{4}, f 4=h^{8}, f 5=h^{16},(-1)+(1) * f 1+(1) * f 3+(-1) * f 1 * f 2+(\right.\right.$

$-1) * f 1 * f 4+(-1) * f 2 * f 3+(1) * f 2 * f 4+(1) * f 1 * f 2 * f 3+(1) * f 1 * f 4 * f 5+(1) * f 2 * f 3 * f 5$

$+(-1) * f 2 * f 4 * f 5+(1) * f 3 * f 4 * f 5+(-1) * f 1 * f 2 * f 3 * f 5+(-1) * f 1 * f 3 * f 4 * f 5+(1) * f 1$

$* f 2 * f 3 * f 4 * f 5))$

$h^{31}-h^{29}+h^{28}-h^{26}+h^{25}-h^{23}+h^{22}+h^{10}-h^{9}+h^{7}-h^{6}+h^{4}-h^{3}+h-1$

(63)

$u_{8}:=\operatorname{sort}\left(\operatorname{subs}\left(f l=h, f 2=h^{2}, f 3=h^{4}, f 4=h^{8}, f 5=h^{16},(85)+(-42) * f l+(7) * f 2+(-72) * f 3\right.\right.$

$+(60) * f 4+(84) * f 5+(-56) * f 1 * f 2+(-46) * f 1 * f 3+(60) * f 1 * f 4+(-42) * f 1 * f 5+($

$-7) * f 2 * f 3+(-7) * f 2 * f 4+(7) * f 2 * f 5+(-72) * f 3 * f 4+(-72) * f 3 * f 5+(60) * f 4 * f 5$

$+(60) * f 1 * f 2 * f 3+(-46) * f 1 * f 2 * f 4+(-56) * f 1 * f 2 * f 5+(-56) * f 1 * f 3 * f 4+(-46) * f 1$

$* f 3 * f 5+(60) * f 1 * f 4 * f 5+(7) * f 2 * f 3 * f 4+(-7) * f 2 * f 3 * f 5+(-7) * f 2 * f 4 * f 5+(-72)$

$* f 3 * f 4 * f 5+(-42) * f 1 * f 2 * f 3 * f 4+(60) * f 1 * f 2 * f 3 * f 5+(-46) * f 1 * f 2 * f 4 * f 5+(-56)$

$* f 1 * f 3 * f 4 * f 5+(7) * f 2 * f 3 * f 4 * f 5+(-42) * f 1 * f 2 * f 3 * f 4 * f 5))$

$-42 h^{31}+7 h^{30}-56 h^{29}-72 h^{28}-46 h^{27}-7 h^{26}+60 h^{25}+60 h^{24}+60 h^{23}-7 h^{22}$

(64)

$-46 h^{21}-72 h^{20}-56 h^{19}+7 h^{18}-42 h^{17}+84 h^{16}-42 h^{15}+7 h^{14}-56 h^{13}$ 
$-72 h^{12}-46 h^{11}-7 h^{10}+60 h^{9}+60 h^{8}+60 h^{7}-7 h^{6}-46 h^{5}-72 h^{4}-56 h^{3}$

$+7 h^{2}-42 h+85$

$u_{9}:=\operatorname{sort}\left(\operatorname{subs}\left(f 1=h, f 2=h^{2}, f 3=h^{4}, f 4=h^{8}, f 5=h^{16},(5)+(-2) * f 1+(-2) * f 2+(-4) * f 4+(4)\right.\right.$

$* f 5+(3) * f 1 * f 2+(-3) * f 1 * f 3+(2) * f 1 * f 4+(-2) * f 1 * f 5+(2) * f 2 * f 3+(2) * f 2 * f 4$

$+(-2) * f 2 * f 5+(-4) * f 4 * f 5+(2) * f 1 * f 2 * f 3+(-3) * f 1 * f 2 * f 4+(3) * f 1 * f 2 * f 5+(3)$

$* f 1 * f 3 * f 4+(-3) * f 1 * f 3 * f 5+(2) * f 1 * f 4 * f 5+(-2) * f 2 * f 3 * f 4+(2) * f 2 * f 3 * f 5+(2)$

$* f 2 * f 4 * f 5+(-2) * f 1 * f 2 * f 3 * f 4+(2) * f 1 * f 2 * f 3 * f 5+(-3) * f 1 * f 2 * f 4 * f 5+(3) * f 1 * f 3$

$* f 4 * f 5+(-2) * f 2 * f 3 * f 4 * f 5+(-2) * f 1 * f 2 * f 3 * f 4 * f 5))$

$-2 h^{31}-2 h^{30}+3 h^{29}-3 h^{27}+2 h^{26}+2 h^{25}-4 h^{24}+2 h^{23}+2 h^{22}-3 h^{21}+3 h^{19}$

(65)

$-2 h^{18}-2 h^{17}+4 h^{16}-2 h^{15}-2 h^{14}+3 h^{13}-3 h^{11}+2 h^{10}+2 h^{9}-4 h^{8}+2 h^{7}$

$+2 h^{6}-3 h^{5}+3 h^{3}-2 h^{2}-2 h+5$

$u_{10}:=\operatorname{sort}\left(\operatorname{subs}\left(f l=h, f 2=h^{2}, f 3=h^{4}, f 4=h^{8}, f 5=h^{16},(73)+(51) * f l+(-72) * f 3+(72) * f 4\right.\right.$

$+(72) * f 5+(-51) * f 1 * f 2+($

$-51) * f 1 * f 3+(51) * f 1 * f 4+(51) * f 1 * f 5+(-72) * f 3 * f 4+(-72) * f 3 * f 5+(72) * f 4 * f 5+($

$51) * f 1 * f 2 * f 3+(-51) * f 1 * f 2 * f 4+(-51) * f 1 * f 2 * f 5+(-51) * f 1 * f 3 * f 4+(-51) * f 1 * f 3 * f 5$

$+($

$51) * f 1 * f 4 * f 5+(-72) * f 3 * f 4 * f 5+(51) * f 1 * f 2 * f 3 * f 4+(51) * f 1 * f 2 * f 3 * f 5+($

$-51) * f 1 * f 2 * f 4 * f 5+(-51) * f 1 * f 3 * f 4 * f 5+(51) * f 1 * f 2 * f 3 * f 4 * f 5))$

$51 h^{31}-51 h^{29}-72 h^{28}-51 h^{27}+51 h^{25}+72 h^{24}+51 h^{23}-51 h^{21}-72 h^{20}-51 h^{19}$

(66)

$+51 h^{17}+72 h^{16}+51 h^{15}-51 h^{13}-72 h^{12}-51 h^{11}+51 h^{9}+72 h^{8}+51 h^{7}$

$-51 h^{5}-72 h^{4}-51 h^{3}+51 h+73$

$u_{11}:=\operatorname{sort}\left(\operatorname{subs}\left(f I=h, f 2=h^{2}, f 3=h^{4}, f 4=h^{8}, f 5=h^{16},(1)+(3) * f 1+(-1) * f 2+(-2) * f 3+(4)\right.\right.$

$* f 4+(-1) * f 1 * f 2+(-2) * f 1 * f 3+(3) * f 1 * f 5+(1) * f 2 * f 3+(1) * f 2 * f 4+(-1) * f 2 * f 5$

$+(-2) * f 3 * f 4+(-2) * f 3 * f 5+(4) * f 4 * f 5+(-2) * f 1 * f 2 * f 4+(-1) * f 1 * f 2 * f 5+(-1)$

$* f 1 * f 3 * f 4+(-2) * f 1 * f 3 * f 5+(-1) * f 2 * f 3 * f 4+(1) * f 2 * f 3 * f 5+(1) * f 2 * f 4 * f 5+(-2)$

$* f 3 * f 4 * f 5+(3) * f 1 * f 2 * f 3 * f 4+(-2) * f 1 * f 2 * f 4 * f 5+(-1) * f 1 * f 3 * f 4 * f 5+(-1) * f 2 * f 3$

$* f 4 * f 5+(3) * f 1 * f 2 * f 3 * f 4 * f 5))$

$3 h^{31}-h^{30}-h^{29}-2 h^{28}-2 h^{27}+h^{26}+4 h^{24}+h^{22}-2 h^{21}-2 h^{20}-h^{19}-h^{18}$

(67)

$+3 h^{17}+3 h^{15}-h^{14}-h^{13}-2 h^{12}-2 h^{11}+h^{10}+4 h^{8}+h^{6}-2 h^{5}-2 h^{4}-h^{3}$

$-h^{2}+3 h+1$

Vamos agora substituir $h$ por $x+1$, encontrando $v_{i}=f_{1}\left(u_{i}\right)$.

$v_{1}:=\operatorname{sort}\left(\operatorname{expand}\left(\operatorname{subs}\left(h=x+1, u_{1}\right)\right) \bmod 2\right)$

$x^{31}+x^{30}+x^{26}+x^{25}+x^{24}+x^{20}+x^{19}+x^{18}+x^{14}+x^{13}+x^{12}+x^{8}+x^{7}+x^{6}+1$

(68)

$v_{2}:=\operatorname{sort}\left(\operatorname{expand}\left(\operatorname{subs}\left(h=x+1, u_{2}\right)\right) \bmod 2\right)$

$$
x^{31}+x^{30}+x^{28}+x^{27}+x^{25}+x^{24}+x^{14}+x^{13}+x^{11}+x^{10}+1
$$

$v_{3}:=\operatorname{sort}\left(\operatorname{expand}\left(\operatorname{subs}\left(h=x+1, u_{3}\right)\right) \bmod 2\right)$

$x^{31}+x^{30}+x^{28}+x^{27}+x^{26}+x^{24}+x^{23}+x^{22}+x^{20}+x^{19}+x^{18}+x^{16}+x^{15}+x^{14}+x^{12}$ 


$$
\begin{aligned}
& +x^{11}+x^{10}+x^{8}+x^{7}+x^{6}+1 \\
& v_{4}:=\operatorname{sort}\left(\operatorname{expand}\left(\operatorname{subs}\left(h=x+1, u_{4}\right)\right) \bmod 2\right) \\
& x^{31}+x^{30}+x^{28}+x^{27}+x^{25}+x^{24}+x^{22}+x^{21}+x^{19}+x^{18}+1 \\
& v_{5}:=\operatorname{sort}\left(\text { expand }\left(\operatorname{subs}\left(h=x+1, u_{5}\right)\right) \bmod 2\right) \\
& x^{30}+x^{29}+x^{27}+x^{26}+x^{24}+x^{23}+x^{21}+x^{20}+x^{14}+x^{13}+x^{12}+x^{8}+x^{7}+x^{6}+1 \\
& v_{6}:=\operatorname{sort}\left(\operatorname{expand}\left(\operatorname{subs}\left(h=x+1, u_{6}\right)\right) \bmod 2\right) \\
& x^{30}+x^{29}+x^{27}+x^{26}+x^{22}+x^{21}+x^{19}+x^{18}+x^{14}+x^{13}+x^{11}+x^{10}+1 \\
& v_{7}:=\operatorname{sort}\left(\operatorname{expand}\left(\operatorname{subs}\left(h=x+1, u_{7}\right)\right) \bmod 2\right) \\
& x^{31}+x^{30}+x^{28}+x^{27}+x^{25}+x^{24}+x^{22}+x^{21}+x^{20}+x^{16}+x^{15}+x^{14}+x^{12}+x^{11}+x^{10} \\
& +x^{8}+x^{7}+x^{6}+1 \\
& v_{8}:=\operatorname{sort}\left(\operatorname{expand}\left(\operatorname{subs}\left(h=x+1, u_{8}\right)\right) \bmod 2\right) \\
& x^{30}+x^{28}+1 \\
& v_{9}:=\operatorname{sort}\left(\operatorname{expand}\left(\operatorname{subs}\left(h=x+1, u_{9}\right)\right) \bmod 2\right) \\
& x^{29}+x^{28}+x^{27}+x^{26}+1 \\
& v_{10}:=\operatorname{sort}\left(\operatorname{expand}\left(\operatorname{subs}\left(h=x+1, u_{10}\right)\right) \bmod 2\right) \\
& x^{31}+x^{30}+1 \\
& v_{11}:=\operatorname{sort}\left(\text { expand }\left(\operatorname{subs}\left(h=x+1, u_{11}\right)\right) \bmod 2\right) \\
& x^{31}+x^{28}+x^{27}+x^{26}+x^{23}+x^{22}+1
\end{aligned}
$$

Vamos encontrar um conjunto multiplicativamente independente que gera $\operatorname{Im}\left(f_{1}\right)=\left\langle h, v_{1}, v_{2}, v_{3}, \ldots, v_{11}\right\rangle$.

$$
\begin{aligned}
& e_{1}:=v_{1} \\
& x^{31}+x^{30}+x^{26}+x^{25}+x^{24}+x^{20}+x^{19}+x^{18}+x^{14}+x^{13}+x^{12}+x^{8}+x^{7}+x^{6}+1 \\
& e_{2}:=v_{2} \\
& x^{31}+x^{30}+x^{28}+x^{27}+x^{25}+x^{24}+x^{14}+x^{13}+x^{11}+x^{10}+1 \\
& e_{3}:=\operatorname{sort}\left(\text { mtaylor }\left(\text { expand }\left(v_{3} \cdot e_{1}^{3} \cdot e_{2}\right), x, 32\right) \bmod 2\right) \\
& x^{31}+x^{30}+x^{29}+x^{24}+x^{15}+x^{14}+1 \\
& e_{4}:=v_{4} \\
& x^{31}+x^{30}+x^{28}+x^{27}+x^{25}+x^{24}+x^{22}+x^{21}+x^{19}+x^{18}+1 \\
& e_{5}:=\operatorname{sort}\left(\text { mtaylor }\left(\text { expand }\left(v_{5} \cdot e_{1}^{3} \cdot e_{4}\right), x, 32\right) \bmod 2\right) \\
& x^{31}+x^{29}+x^{28}+x^{24}+x^{23}+x^{22}+1 \\
& e_{6}:=\operatorname{sort}\left(\text { mtaylor }\left(\text { expand }\left(v_{6} \cdot e_{2}^{3} \cdot e_{4}\right), x, 32\right) \bmod 2\right) \#=v_{9} \\
& x^{29}+x^{28}+x^{27}+x^{26}+1 \\
& e_{7}:=\operatorname{sort}\left(\text { mtaylor }\left(\text { expand }\left(v_{7} \cdot e_{1}^{7} \cdot e_{2} \cdot e_{3}^{3} \cdot e_{4} \cdot e_{5} \cdot e_{6}\right), x, 32\right) \bmod 2\right) \#=v_{10} \\
& x^{31}+x^{30}+1
\end{aligned}
$$


$\operatorname{sort}\left(\right.$ mtaylor $\left(\right.$ expand $\left.\left.\left(v_{8} \cdot e_{3}^{2}\right), x, 32\right) \bmod 2\right) \#=v_{1}^{6} v_{2}^{2} v_{3}^{2} v_{8}$

1

sort $\left(\right.$ mtaylor $\left(\right.$ expand $\left.\left.\left(v_{9} \cdot e_{6}\right), x, 32\right) \bmod 2\right) \#=v_{2}^{3} v_{4} v_{6} v_{9}$

1

$\operatorname{sort}\left(\right.$ mtaylor (expand $\left.\left.\left(v_{10} \cdot e_{7}\right), x, 32\right) \bmod 2\right) \#=v_{1}^{3} v_{2}^{-1} v_{3}^{3} v_{4} v_{5} v_{6} v_{7} v_{10}$

1

$\operatorname{sort}\left(\right.$ mtaylor $\left(\right.$ expand $\left.\left.\left(v_{11} \cdot e_{5} \cdot e_{1}^{4} \cdot e_{6}\right), x, 32\right) \bmod 2\right) \quad \#=v_{1}^{7} \cdot v_{2}^{3} \cdot v_{4}^{2} \cdot v_{5} \cdot v_{6} v_{11}=v_{1}^{-1} \cdot v_{2}^{-1} \cdot v_{5} \cdot v_{6} v_{11}$

Elementos do núcleo:

sort (mtaylor (expand $\left.\left.\left(v_{1}^{6} v_{2}^{2} v_{3}^{2} v_{8}\right), x, 32\right) \bmod 2\right)$

sort (mtaylor (expand $\left.\left.\left(v_{2}^{3} v_{4} v_{6} v_{9}\right), x, 32\right) \bmod 2\right)$

1

(91)

sort (mtaylor (expand $\left.\left.\left(v_{1}^{3} v_{2}^{-1} v_{3}^{3} v_{4} v_{5} v_{6} v_{7} v_{10}\right), x, 32\right) \bmod 2\right)$

$\operatorname{sort}\left(\right.$ mtaylor (expand $\left.\left.\left(v_{1}^{-1} \cdot v_{2}^{-1} \cdot v_{5} \cdot v_{6} \cdot v_{11}\right), x, 32\right) \bmod 2\right)$

$\operatorname{sort}\left(\operatorname{mtaylor}\left(\operatorname{expand}\left(v_{1}^{4} \cdot v_{3}^{4}\right), x, 32\right) \bmod 2\right)$

$\operatorname{sort}\left(\right.$ mtaylor $\left.\left(\operatorname{expand}\left(v_{5}^{2} \cdot v_{1}^{-2}\right), x, 32\right) \bmod 2\right)$

sort $\left(\right.$ mtaylor (expand $\left.\left.\left(v_{6}^{2} \cdot v_{2}^{2}\right), x, 32\right) \bmod 2\right)$

$\operatorname{sort}\left(\right.$ mtaylor $\left(\right.$ expand $\left.\left.\left(v_{1}^{-2} \cdot v_{2}^{2} \cdot v_{3}^{-2} \cdot v_{5}^{2} \cdot v_{6}^{2} \cdot v_{7}^{2}\right), x, 32\right) \bmod 2\right)$

1

$\operatorname{Logo} \operatorname{Im}\left(f_{1}\right)=\left\langle h, v_{1}, v_{2}, \ldots, v_{11}\right\rangle=\langle h\rangle \times\left\langle e_{1}\right\rangle \times\left\langle e_{2}\right\rangle \times \ldots \times\left\langle e_{7}\right\rangle$.

\section{Caso $2^{7}$ :}

$u_{i}$ é a unidade simétrica normalizada de $\mathbb{Z} C_{64}$.

$$
\begin{aligned}
u_{1}:= & \operatorname{sort}\left(\operatorname { s u b s } \left(f 1=h, f 2=h^{2}, f 3=h^{4}, f 4=h^{8}, f 5=h^{16}, f 6=h^{32},(1)+(-1) * f 2+(1) * f 3+(-2) * f 4\right.\right. \\
& +(1) * f 5+(-2) * f 6+(1) * f 1 * f 2+(-2) * f 1 * f 3+(1) * f 1 * f 4+(-2) * f 1 * f 5+(1) * f 1 \\
& * f 6+(1) * f 2 * f 3+(1) * f 2 * f 4+(1) * f 2 * f 5+(1) * f 2 * f 6+(1) * f 3 * f 4+(-2) * f 3 * f 5 \\
& +(1) * f 3 * f 6+(1) * f 4 * f 5+(1) * f 4 * f 6+(1) * f 5 * f 6+(1) * f 1 * f 2 * f 3+(-2) * f 1 * f 2 * f 4
\end{aligned}
$$


$+(1) * f 1 * f 2 * f 5+(-2) * f 1 * f 2 * f 6+(1) * f 1 * f 3 * f 4+(1) * f 1 * f 3 * f 5+(1) * f 1 * f 3 * f 6$ $+(1) * f 1 * f 4 * f 5+(-2) * f 1 * f 4 * f 6+(1) * f 1 * f 5 * f 6+(-2) * f 2 * f 3 * f 4+(1) * f 2 * f 3 * f 5$ $+(-2) * f 2 * f 3 * f 6+(-2) * f 2 * f 4 * f 5+(1) * f 2 * f 4 * f 6+(-2) * f 2 * f 5 * f 6+(1) * f 3 * f 4 * f 5$ $+(-2) * f 3 * f 4 * f 6+(1) * f 3 * f 5 * f 6+(-2) * f 4 * f 5 * f 6+(1) * f 1 * f 2 * f 3 * f 4+(-2) * f 1 * f 2$ $* f 3 * f 5+(1) * f 1 * f 2 * f 3 * f 6+(1) * f 1 * f 2 * f 4 * f 5+(1) * f 1 * f 2 * f 4 * f 6+(1) * f 1 * f 2 * f 5 * f 6$ $+(-2) * f 1 * f 3 * f 4 * f 5+(1) * f 1 * f 3 * f 4 * f 6+(-2) * f 1 * f 3 * f 5 * f 6+(1) * f 1 * f 4 * f 5 * f 6$ $+(1) * f 2 * f 3 * f 4 * f 5+(1) * f 2 * f 3 * f 4 * f 6+(1) * f 2 * f 3 * f 5 * f 6+(1) * f 2 * f 4 * f 5 * f 6+(1)$ $* f 3 * f 4 * f 5 * f 6+(1) * f 1 * f 2 * f 3 * f 4 * f 5+(-2) * f 1 * f 2 * f 3 * f 4 * f b+(1) * f 1 * f 2 * f 3 * f 5 * f b$ $+(-2) * f 1 * f 2 * f 4 * f 5 * f 6+(1) * f 1 * f 3 * f 4 * f 5 * f 6+(-1) * f 2 * f 3 * f 4 * f 5 * f 6))$ $-h^{62}+h^{61}+h^{60}-2 h^{59}+h^{58}+h^{57}-2 h^{56}+h^{55}+h^{54}-2 h^{53}+h^{52}+h^{51}-2 h^{50}$

$+h^{49}+h^{48}-2 h^{47}+h^{46}+h^{45}-2 h^{44}+h^{43}+h^{42}-2 h^{41}+h^{40}+h^{39}-2 h^{38}$

$+h^{37}+h^{36}-2 h^{35}+h^{34}+h^{33}-2 h^{32}+h^{31}+h^{30}-2 h^{29}+h^{28}+h^{27}-2 h^{26}$

$+h^{25}+h^{24}-2 h^{23}+h^{22}+h^{21}-2 h^{20}+h^{19}+h^{18}-2 h^{17}+h^{16}+h^{15}-2 h^{14}$

$+h^{13}+h^{12}-2 h^{11}+h^{10}+h^{9}-2 h^{8}+h^{7}+h^{6}-2 h^{5}+h^{4}+h^{3}-h^{2}+1$

$u_{2}:=\operatorname{sort}\left(\operatorname{subs}\left(f 1=h, f 2=h^{2}, f 3=h^{4}, f 4=h^{8}, f 5=h^{16}, f 6=h^{32},(1)+(-1) * f 2+(-1) * f 4+(1) * f 5\right.\right.$

$+(-2) * f 6+(1) * f 1 * f 2+(-1) * f 1 * f 3+(1) * f 1 * f 4+(-2) * f 1 * f 5+(1) * f 1 * f 6+(1)$ $* f 2 * f 3+(1) * f 2 * f 4+(1) * f 2 * f 5+(1) * f 2 * f 6+(1) * f 3 * f 4+(-2) * f 3 * f 5+(1) * f 3 * f 6$ $+(1) * f 4 * f 5+(1) * f 4 * f 6+(1) * f 5 * f 6+(-2) * f 1 * f 2 * f 4+(1) * f 1 * f 2 * f 5+(-2) * f 1 * f 2$ $* f 6+(1) * f 1 * f 3 * f 4+(1) * f 1 * f 3 * f 5+(1) * f 1 * f 3 * f 6+(1) * f 1 * f 4 * f 5+(-2) * f 1 * f 4 * f 6$ $+(1) * f 1 * f 5 * f 6+(-2) * f 2 * f 3 * f 4+(1) * f 2 * f 3 * f 5+(-2) * f 2 * f 3 * f 6+(-2) * f 2 * f 4 * f 5$ $+(1) * f 2 * f 4 * f 6+(-2) * f 2 * f 5 * f 6+(1) * f 3 * f 4 * f 5+(-2) * f 3 * f 4 * f 6+(1) * f 3 * f 5 * f 6$ $+(-1) * f 4 * f 5 * f 6+(1) * f 1 * f 2 * f 3 * f 4+(-2) * f 1 * f 2 * f 3 * f 5+(1) * f 1 * f 2 * f 3 * f 6+(1)$ $* f 1 * f 2 * f 4 * f 5+(1) * f 1 * f 2 * f 4 * f 6+(1) * f 1 * f 2 * f 5 * f 6+(-2) * f 1 * f 3 * f 4 * f 5+(1) * f 1 * f 3$ $* f 4 * f 6+(-2) * f 1 * f 3 * f 5 * f 6+(1) * f 2 * f 3 * f 4 * f 5+(1) * f 2 * f 3 * f 4 * f 6+(1) * f 2 * f 3 * f 5 * f 6$ $+(1) * f 2 * f 4 * f 5 * f 6+(1) * f 1 * f 2 * f 3 * f 4 * f 5+(-2) * f 1 * f 2 * f 3 * f 4 * f 6+(1) * f 1 * f 2 * f 3 * f 5$

$* f 6+(-1) * f 1 * f 2 * f 4 * f 5 * f 6+(1) * f 1 * f 3 * f 4 * f 5 * f 6+(-1) * f 2 * f 3 * f 4 * f 5 * f 6))$

$-h^{62}+h^{61}-h^{59}+h^{58}-h^{56}+h^{55}+h^{54}-2 h^{53}+h^{52}+h^{51}-2 h^{50}+h^{49}+h^{48}$

(99)

$-2 h^{47}+h^{46}+h^{45}-2 h^{44}+h^{43}+h^{42}-2 h^{41}+h^{40}+h^{39}-2 h^{38}+h^{37}+h^{36}$

$-2 h^{35}+h^{34}+h^{33}-2 h^{32}+h^{31}+h^{30}-2 h^{29}+h^{28}+h^{27}-2 h^{26}+h^{25}+h^{24}$

$-2 h^{23}+h^{22}+h^{21}-2 h^{20}+h^{19}+h^{18}-2 h^{17}+h^{16}+h^{15}-2 h^{14}+h^{13}+h^{12}$

$-2 h^{11}+h^{10}+h^{9}-h^{8}+h^{6}-h^{5}+h^{3}-h^{2}+1$

$u_{3}:=\operatorname{sort}\left(\operatorname{subs}\left(f l=h, f 2=h^{2}, f 3=h^{4}, f 4=h^{8}, f 5=h^{16}, f 6=h^{32},(1)+(-1) * f 2+(-1) * f 4+(-2)\right.\right.$

$* f 6+(1) * f 1 * f 2+(-1) * f 1 * f 3+(1) * f 1 * f 4+(-1) * f 1 * f 5+(1) * f 1 * f 6+(1) * f 2 * f 3$

$+(1) * f 2 * f 5+(1) * f 2 * f 6+(1) * f 3 * f 4+(-1) * f 3 * f 5+(1) * f 3 * f 6+(1) * f 4 * f 5+(1)$

$* f 4 * f 6+(-1) * f 1 * f 2 * f 4+(-2) * f 1 * f 2 * f 6+(1) * f 1 * f 3 * f 5+(1) * f 1 * f 3 * f 6+(-1) * f 1$

$* f 4 * f 6+(1) * f 1 * f 5 * f 6+(-1) * f 2 * f 3 * f 4+(-1) * f 2 * f 3 * f 6+(-1) * f 2 * f 4 * f 5+(-1) * f 2$

$* f 5 * f 6+(1) * f 3 * f 4 * f 5+(-1) * f 3 * f 4 * f 6+(1) * f 3 * f 5 * f 6+(-1) * f 4 * f 5 * f 6+(1) * f 1$

$* f 2 * f 3 * f 4+(-1) * f 1 * f 2 * f 3 * f 5+(1) * f 1 * f 2 * f 4 * f 5+(1) * f 1 * f 2 * f 4 * f 6+(-2) * f 1 * f 3$

$* f 4 * f 5+(-1) * f 1 * f 3 * f 5 * f 6+(1) * f 2 * f 3 * f 4 * f 5+(1) * f 2 * f 3 * f 4 * f b+(1) * f 2 * f 4 * f 5 * f b$ $+(1) * f 1 * f 2 * f 3 * f 4 * f 5+(-1) * f 1 * f 2 * f 3 * f 4 * f 6+(1) * f 1 * f 2 * f 3 * f 5 * f 6+(-1) * f 1 * f 2$

$* f 4 * f 5 * f 6+(1) * f 1 * f 3 * f 4 * f 5 * f 6+(-1) * f 2 * f 3 * f 4 * f 5 * f 6))$

$-h^{62}+h^{61}-h^{59}+h^{58}-h^{56}+h^{55}-h^{53}+h^{52}-h^{50}+h^{49}-h^{47}+h^{46}-h^{44}+h^{43}$

(100) 
$-h^{41}+h^{40}-h^{38}+h^{37}+h^{36}-2 h^{35}+h^{34}+h^{33}-2 h^{32}+h^{31}+h^{30}-2 h^{29}$

$+h^{28}+h^{27}-h^{26}+h^{24}-h^{23}+h^{21}-h^{20}+h^{18}-h^{17}+h^{15}-h^{14}+h^{12}-h^{11}$

$+h^{9}-h^{8}+h^{6}-h^{5}+h^{3}-h^{2}+1$

$u_{4}:=\operatorname{sort}\left(\operatorname{subs}\left(f 1=h, f 2=h^{2}, f 3=h^{4}, f 4=h^{8}, f 5=h^{16}, f 6=h^{32},(-1)+(1) * f 1+(1) * f 3+(1) * f 5\right.\right.$

$+(-1) * f 1 * f 2+(-1) * f 1 * f 4+(-1) * f 2 * f 3+(1) * f 2 * f 4+(-1) * f 3 * f 4+(1) * f 5 * f 6$

$+(1) * f 1 * f 2 * f 3+(1) * f 1 * f 3 * f 4+(-1) * f 1 * f 5 * f 6+(-1) * f 3 * f 5 * f 6+(-1) * f 1 * f 2 * f 3$

$* f 4+(1) * f 1 * f 2 * f 5 * f 6+(1) * f 1 * f 4 * f 5 * f 6+(1) * f 2 * f 3 * f 5 * f 6+(-1) * f 2 * f 4 * f 5 * f 6$

$+(1) * f 3 * f 4 * f 5 * f 6+(-1) * f 1 * f 2 * f 3 * f 5 * f b+(-1) * f 1 * f 3 * f 4 * f 5 * f 6+(1) * f 1 * f 2 * f 3$

$* f 4 * f 5 * f 6))$

$h^{63}-h^{61}+h^{60}-h^{58}+h^{57}-h^{55}+h^{54}-h^{52}+h^{51}-h^{49}+h^{48}+h^{16}-h^{15}+h^{13}$

(101)

$-h^{12}+h^{10}-h^{9}+h^{7}-h^{6}+h^{4}-h^{3}+h-1$

$u_{5}:=\operatorname{sort}\left(\operatorname{subs}\left(f 1=h, f 2=h^{2}, f 3=h^{4}, f 4=h^{8}, f 5=h^{16}, f 6=h^{32},(1)+(-1) * f 2+(-1) * f 4+(1) * f 1\right.\right.$

$* f 2+(-1) * f 1 * f 3+(1) * f 1 * f 4+(1) * f 2 * f 3+(1) * f 3 * f 4+(-1) * f 1 * f 2 * f 4+(1) * f 3 * f 5$

$* f b+(-1) * f 4 * f 5 * f b+(-1) * f 1 * f 3 * f 5 * f 6+(1) * f 2 * f 4 * f 5 * f 6+(1) * f 1 * f 2 * f 3 * f 5 * f b$

$+(-1) * f 1 * f 2 * f 4 * f 5 * f 6+(1) * f 1 * f 3 * f 4 * f 5 * f 6+(-1) * f 2 * f 3 * f 4 * f 5 * f 6))$

$-h^{62}+h^{61}-h^{59}+h^{58}-h^{56}+h^{55}-h^{53}+h^{52}+h^{12}-h^{11}+h^{9}-h^{8}+h^{6}-h^{5}+h^{3}$

(102)

$-h^{2}+1$

$u_{6}:=\operatorname{sort}\left(\operatorname{subs}\left(f l=h, f 2=h^{2}, f 3=h^{4}, f 4=h^{8}, f 5=h^{16}, f 6=h^{32},(1)+(-1) * f 2+(-1) * f 4+(1) * f 1\right.\right.$

$* f 2+(-1) * f 1 * f 3+(1) * f 1 * f 4+(-1) * f 1 * f 5+(1) * f 2 * f 3+(1) * f 2 * f 5+(1) * f 3 * f 4$

$+(-1) * f 3 * f 5+(1) * f 4 * f 5+(1) * f 4 * f 6+(-1) * f 1 * f 2 * f 4+(1) * f 1 * f 3 * f 5+(-1) * f 1$

$* f 4 * f 6+(1) * f 1 * f 5 * f b+(-1) * f 2 * f 3 * f 4+(-1) * f 2 * f 5 * f b+(-1) * f 3 * f 4 * f b+(1) * f 3$

$* f 5 * f 6+(-1) * f 4 * f 5 * f 6+(1) * f 1 * f 2 * f 3 * f 4+(-1) * f 1 * f 2 * f 3 * f 5+(1) * f 1 * f 2 * f 4 * f 6$

$+(-1) * f 1 * f 3 * f 5 * f 6+(1) * f 2 * f 3 * f 4 * f 6+(1) * f 2 * f 4 * f 5 * f 6+(-1) * f 1 * f 2 * f 3 * f 4 * f 6$

$+(1) * f 1 * f 2 * f 3 * f 5 * f 6+(-1) * f 1 * f 2 * f 4 * f 5 * f 6+(1) * f 1 * f 3 * f 4 * f 5 * f 6+(-1) * f 2 * f 3$

$* f 4 * f 5 * f b))$

$-h^{62}+h^{61}-h^{59}+h^{58}-h^{56}+h^{55}-h^{53}+h^{52}-h^{50}+h^{49}-h^{47}+h^{46}-h^{44}+h^{43}$

(103)

$-h^{41}+h^{40}+h^{24}-h^{23}+h^{21}-h^{20}+h^{18}-h^{17}+h^{15}-h^{14}+h^{12}-h^{11}+h^{9}$

$-h^{8}+h^{6}-h^{5}+h^{3}-h^{2}+1$

$u_{7}:=\operatorname{sort}\left(\operatorname{subs}\left(f l=h, f 2=h^{2}, f 3=h^{4}, f 4=h^{8}, f 5=h^{16}, f 6=h^{32},(-1)+(1) * f 1+(1) * f 3+(-1) * f 1\right.\right.$

$* f 2+(-1) * f 1 * f 4+(-1) * f 2 * f 3+(1) * f 2 * f 4+(1) * f 1 * f 2 * f 3+(1) * f 1 * f 4 * f 5 * f 6+(1)$

$* f 2 * f 3 * f 5 * f 6+(-1) * f 2 * f 4 * f 5 * f 6+(1) * f 3 * f 4 * f 5 * f 6+(-1) * f 1 * f 2 * f 3 * f 5 * f 6+(-1)$

$* f 1 * f 3 * f 4 * f 5 * f 6+(1) * f 1 * f 2 * f 3 * f 4 * f 5 * f 6))$

$h^{63}-h^{61}+h^{60}-h^{58}+h^{57}-h^{55}+h^{54}+h^{10}-h^{9}+h^{7}-h^{6}+h^{4}-h^{3}+h-1$

(104)

$u_{8}:=\operatorname{sort}\left(\operatorname{subs}\left(f 1=h, f 2=h^{2}, f 3=h^{4}, f 4=h^{8}, f 5=h^{16}, f 6=h^{32},(1)+(-1) * f 2+(-1) * f 4+(1) * f 1\right.\right.$

$* f 2+(-1) * f 1 * f 3+(1) * f 1 * f 4+(-1) * f 1 * f 5+(1) * f 2 * f 3+(1) * f 2 * f 5+(1) * f 2 * f 6$

$+(1) * f 3 * f 4+(-1) * f 3 * f 5+(1) * f 4 * f 5+(1) * f 4 * f 6+(-1) * f 1 * f 2 * f 4+(-1) * f 1 * f 2$

$* f 6+(1) * f 1 * f 3 * f 5+(1) * f 1 * f 3 * f 6+(-1) * f 1 * f 4 * f 6+(1) * f 1 * f 5 * f 6+(-1) * f 2 * f 3$

$* f 4+(-1) * f 2 * f 3 * f 6+(-1) * f 2 * f 4 * f 5+(-1) * f 2 * f 5 * f 6+(-1) * f 3 * f 4 * f 6+(1) * f 3 * f 5$

$* f 6+(-1) * f 4 * f 5 * f 6+(1) * f 1 * f 2 * f 3 * f 4+(-1) * f 1 * f 2 * f 3 * f 5+(1) * f 1 * f 2 * f 4 * f 5$

$+(1) * f 1 * f 2 * f 4 * f 6+(-1) * f 1 * f 3 * f 4 * f 5+(-1) * f 1 * f 3 * f 5 * f 6+(1) * f 2 * f 3 * f 4 * f 5$

$+(1) * f 2 * f 3 * f 4 * f 6+(1) * f 2 * f 4 * f 5 * f 6+(-1) * f 1 * f 2 * f 3 * f 4 * f 6+(1) * f 1 * f 2 * f 3 * f 5 * f 6$ 


$$
\begin{aligned}
& +(-1) * f 1 * f 2 * f 4 * f 5 * f 6+(1) * f 1 * f 3 * f 4 * f 5 * f 6+(-1) * f 2 * f 3 * f 4 * f 5 * f 6)) \\
& -h^{62}+h^{61}-h^{59}+h^{58}-h^{56}+h^{55}-h^{53}+h^{52}-h^{50}+h^{49}-h^{47}+h^{46}-h^{44}+h^{43} \\
& -h^{41}+h^{40}-h^{38}+h^{37}-h^{35}+h^{34}+h^{30}-h^{29}+h^{27}-h^{26}+h^{24}-h^{23}+h^{21} \\
& -h^{20}+h^{18}-h^{17}+h^{15}-h^{14}+h^{12}-h^{11}+h^{9}-h^{8}+h^{6}-h^{5}+h^{3}-h^{2}+1 \\
& u_{9}:=\operatorname{sort}\left(\operatorname { s u b s } \left(f 1=h, f 2=h^{2}, f 3=h^{4}, f 4=h^{8}, f 5=h^{16}, f 6=h^{32},(-1)+(1) * f 1+(1) * f 3+(1) * f 5\right.\right. \\
& +(-1) * f 1 * f 2+(-1) * f 1 * f 4+(-1) * f 2 * f 3+(1) * f 2 * f 4+(-1) * f 2 * f 5+(-1) * f 3 * f 4 \\
& +(1) * f 3 * f 6+(-1) * f 4 * f 5+(-1) * f 4 * f 6+(1) * f 5 * f 6+(1) * f 1 * f 2 * f 3+(1) * f 1 * f 2 * f 5 \\
& +(1) * f 1 * f 3 * f 4+(-1) * f 1 * f 3 * f 5+(-1) * f 1 * f 3 * f 6+(1) * f 1 * f 4 * f 5+(-1) * f 1 * f 5 * f 6 \\
& +(1) * f 2 * f 3 * f 5+(1) * f 2 * f 4 * f 6+(1) * f 3 * f 4 * f 5+(-1) * f 3 * f 5 * f 6+(-1) * f 1 * f 2 * f 3 \\
& * f 4+(1) * f 1 * f 2 * f 3 * f 6+(-1) * f 1 * f 2 * f 4 * f 5+(-1) * f 1 * f 2 * f 4 * f 6+(1) * f 1 * f 2 * f 5 * f 6 \\
& +(1) * f 1 * f 3 * f 4 * f 6+(1) * f 1 * f 4 * f 5 * f 6+(-1) * f 2 * f 3 * f 4 * f 6+(1) * f 2 * f 3 * f 5 * f 6+( \\
& -1) * f 2 * f 4 * f 5 * f 6+(1) * f 3 * f 4 * f 5 * f 6+(-1) * f 1 * f 2 * f 3 * f 5 * f 6+(-1) * f 1 * f 3 * f 4 * f 5 * f 6 \\
& +(1) * f 1 * f 2 * f 3 * f 4 * f 5 * f 6)) \\
& h^{63}-h^{61}+h^{60}-h^{58}+h^{57}-h^{55}+h^{54}-h^{52}+h^{51}-h^{49}+h^{48}-h^{46}+h^{45}-h^{43} \\
& +h^{42}-h^{40}+h^{39}-h^{37}+h^{36}+h^{28}-h^{27}+h^{25}-h^{24}+h^{22}-h^{21}+h^{19}-h^{18} \\
& +h^{16}-h^{15}+h^{13}-h^{12}+h^{10}-h^{9}+h^{7}-h^{6}+h^{4}-h^{3}+h-1 \\
& u_{10}:=\operatorname{sort}\left(\operatorname { s u b s } \left(f l=h, f 2=h^{2}, f 3=h^{4}, f 4=h^{8}, f 5=h^{16}, f 6=h^{32},(-1)+(1) * f 1+(1) * f 3+(1) * f 5\right.\right. \\
& +(-1) * f 1 * f 2+(-1) * f 1 * f 4+(-1) * f 2 * f 3+(1) * f 2 * f 4+(-1) * f 2 * f 5+(-1) * f 3 * f 4 \\
& +(1) * f 5 * f 6+(1) * f 1 * f 2 * f 3+(1) * f 1 * f 2 * f 5+(1) * f 1 * f 3 * f 4+(-1) * f 1 * f 3 * f 5+(-1) \\
& * f 1 * f 5 * f 6+(1) * f 2 * f 3 * f 5+(1) * f 2 * f 4 * f 6+(-1) * f 3 * f 5 * f 6+(-1) * f 1 * f 2 * f 3 * f 4+( \\
& -1) * f 1 * f 2 * f 4 * f 6+(1) * f 1 * f 2 * f 5 * f 6+(1) * f 1 * f 3 * f 4 * f 6+(1) * f 1 * f 4 * f 5 * f 6+(-1) \\
& * f 2 * f 3 * f 4 * f 6+(1) * f 2 * f 3 * f 5 * f 6+(-1) * f 2 * f 4 * f 5 * f 6+(1) * f 3 * f 4 * f 5 * f 6+(-1) * f 1 \\
& * f 2 * f 3 * f 5 * f 6+(-1) * f 1 * f 3 * f 4 * f 5 * f 6+(1) * f 1 * f 2 * f 3 * f 4 * f 5 * f \sigma)) \\
& h^{63}-h^{61}+h^{60}-h^{58}+h^{57}-h^{55}+h^{54}-h^{52}+h^{51}-h^{49}+h^{48}-h^{46}+h^{45}-h^{43} \\
& +h^{42}+h^{22}-h^{21}+h^{19}-h^{18}+h^{16}-h^{15}+h^{13}-h^{12}+h^{10}-h^{9}+h^{7}-h^{6}+h^{4} \\
& -h^{3}+h-1 \\
& u_{11}:=\operatorname{sort}\left(\operatorname { s u b s } \left(f l=h, f 2=h^{2}, f 3=h^{4}, f 4=h^{8}, f 5=h^{16}, f 6=h^{32},(-1)+(1) * f 1+(1) * f 3+(-1)\right.\right. \\
& * f 1 * f 2+(1) * f 3 * f 4 * f 5 * f 6+(-1) * f 1 * f 3 * f 4 * f 5 * f 6+(1) * f 1 * f 2 * f 3 * f 4 * f 5 * f 6)) \\
& h^{63}-h^{61}+h^{60}+h^{4}-h^{3}+h-1
\end{aligned}
$$




$$
\begin{aligned}
-h^{62} & +h^{61}-h^{59}+h^{58}-h^{56}+h^{55}-h^{53}+h^{52}-h^{50}+h^{49}+h^{48}-2 h^{47}+h^{46}+h^{45} \\
& -2 h^{44}+h^{43}+h^{42}-2 h^{41}+h^{40}+h^{39}-2 h^{38}+h^{37}+h^{36}-2 h^{35}+h^{34}+h^{33} \\
& -2 h^{32}+h^{31}+h^{30}-2 h^{29}+h^{28}+h^{27}-2 h^{26}+h^{25}+h^{24}-2 h^{23}+h^{22}+h^{21} \\
& -2 h^{20}+h^{19}+h^{18}-2 h^{17}+h^{16}+h^{15}-h^{14}+h^{12}-h^{11}+h^{9}-h^{8}+h^{6}-h^{5} \\
& +h^{3}-h^{2}+1 \\
u_{13}: & =\operatorname{sort}\left(\operatorname { s u b s } \left(f 1=h, f 2=h^{2}, f 3=h^{4}, f 4=h^{8}, f 5=h^{16}, f 6=h^{32},(1)+(-1) * f 2+(-1) * f 4+(1)\right.\right.
\end{aligned}
$$$$
* f 1 * f 2+(-1) * f 1 * f 3+(1) * f 1 * f 4+(-1) * f 1 * f 5+(1) * f 2 * f 3+(1) * f 2 * f 5+(1) * f 3 * f 4
$$$$
+(-1) * f 1 * f 2 * f 4+(1) * f 1 * f 5 * f 6+(-1) * f 2 * f 3 * f 4+(-1) * f 2 * f 5 * f 6+(1) * f 3 * f 5 * f 6
$$$$
+(-1) * f 4 * f 5 * f 6+(1) * f 1 * f 2 * f 3 * f 4+(-1) * f 1 * f 3 * f 5 * f 6+(1) * f 2 * f 3 * f 4 * f 6+(1)
$$$$
* f 2 * f 4 * f 5 * f 6+(-1) * f 1 * f 2 * f 3 * f 4 * f 6+(1) * f 1 * f 2 * f 3 * f 5 * f 6+(-1) * f 1 * f 2 * f 4 * f 5 * f 6
$$$$
+(1) * f 1 * f 3 * f 4 * f 5 * f 6+(-1) * f 2 * f 3 * f 4 * f 5 * f 6))
$$$$
-h^{62}+h^{61}-h^{59}+h^{58}-h^{56}+h^{55}-h^{53}+h^{52}-h^{50}+h^{49}-h^{47}+h^{46}+h^{18}-h^{17}
$$$$
+h^{15}-h^{14}+h^{12}-h^{11}+h^{9}-h^{8}+h^{6}-h^{5}+h^{3}-h^{2}+1
$$

$u_{14}:=\operatorname{sort}\left(\operatorname{subs}\left(f 1=h, f 2=h^{2}, f 3=h^{4}, f 4=h^{8}, f 5=h^{16}, f 6=h^{32},(1)+(-1) * f 2+(1) * f 1 * f 2+(\right.\right.$

$-1) * f 1 * f 3+(1) * f 2 * f 3+(1) * f 2 * f 4 * f 5 * f 6+(-1) * f 1 * f 2 * f 4 * f 5 * f 6+(1) * f 1 * f 3 * f 4$

$* f 5 * f 6+(-1) * f 2 * f 3 * f 4 * f 5 * f 6))$

$$
-h^{62}+h^{61}-h^{59}+h^{58}+h^{6}-h^{5}+h^{3}-h^{2}+1
$$

$u_{15}:=\operatorname{sort}\left(\operatorname{subs}\left(f l=h, f 2=h^{2}, f 3=h^{4}, f 4=h^{8}, f 5=h^{16}, f 6=h^{32},(1)+(-1) * f 2+(-1) * f 4+(-2)\right.\right.$

$* f 6+(1) * f 1 * f 2+(-1) * f 1 * f 3+(1) * f 1 * f 4+(-1) * f 1 * f 5+(1) * f 1 * f 6+(1) * f 2 * f 3$

$+(1) * f 2 * f 5+(1) * f 2 * f 6+(1) * f 3 * f 4+(-1) * f 3 * f 5+(1) * f 3 * f 6+(1) * f 4 * f 5+(1)$

$* f 4 * f 6+(-1) * f 1 * f 2 * f 4+(-2) * f 1 * f 2 * f 6+(1) * f 1 * f 3 * f 5+(1) * f 1 * f 3 * f 6+(1) * f 1$

$* f 4 * f 5+(-2) * f 1 * f 4 * f 6+(1) * f 1 * f 5 * f 6+(-1) * f 2 * f 3 * f 4+(1) * f 2 * f 3 * f 5+(-2) * f 2$

$* f 3 * f 6+(-2) * f 2 * f 4 * f 5+(1) * f 2 * f 4 * f 6+(-1) * f 2 * f 5 * f 6+(1) * f 3 * f 4 * f 5+(-1) * f 3$

$* f 4 * f 6+(1) * f 3 * f 5 * f 6+(-1) * f 4 * f 5 * f 6+(1) * f 1 * f 2 * f 3 * f 4+(-2) * f 1 * f 2 * f 3 * f 5$

$+(1) * f 1 * f 2 * f 3 * f 6+(1) * f 1 * f 2 * f 4 * f 5+(1) * f 1 * f 2 * f 4 * f 6+(-2) * f 1 * f 3 * f 4 * f 5+($

$-1) * f 1 * f 3 * f 5 * f 6+(1) * f 2 * f 3 * f 4 * f 5+(1) * f 2 * f 3 * f 4 * f 6+(1) * f 2 * f 4 * f 5 * f 6+(1) * f 1$

$* f 2 * f 3 * f 4 * f 5+(-1) * f 1 * f 2 * f 3 * f 4 * f 6+(1) * f 1 * f 2 * f 3 * f 5 * f 6+(-1) * f 1 * f 2 * f 4 * f 5 * f 6$

$+(1) * f 1 * f 3 * f 4 * f 5 * f 6+(-1) * f 2 * f 3 * f 4 * f 5 * f 6))$

$-h^{62}+h^{61}-h^{59}+h^{58}-h^{56}+h^{55}-h^{53}+h^{52}-h^{50}+h^{49}-h^{47}+h^{46}-h^{44}+h^{43}$

(112)

$+h^{42}-2 h^{41}+h^{40}+h^{39}-2 h^{38}+h^{37}+h^{36}-2 h^{35}+h^{34}+h^{33}-2 h^{32}+h^{31}$

$+h^{30}-2 h^{29}+h^{28}+h^{27}-2 h^{26}+h^{25}+h^{24}-2 h^{23}+h^{22}+h^{21}-h^{20}+h^{18}$

$-h^{17}+h^{15}-h^{14}+h^{12}-h^{11}+h^{9}-h^{8}+h^{6}-h^{5}+h^{3}-h^{2}+1$

$u_{16}:=\operatorname{sort}\left(\operatorname{subs}\left(f l=h, f 2=h^{2}, f 3=h^{4}, f 4=h^{8}, f 5=h^{16}, f 6=h^{32}\right.\right.$, expand $\left(1+(1+f 6) 2^{-1}\right.$

$\cdot((37545128)+(7370552) * f 1+(-34596492) * f 2+(26393294) * f 3+(81392) * f 4+($

$-37374984) * f 5+(-20868296) * f 1 * f 2+(31073760) * f l * f 3+(36797216) * f 1 * f 4+($

$-7256176) * f 1 * f 5+(-14341040) * f 2 * f 3+(14338500) * f 2 * f 4+(34599032) * f 2 * f 5+($

$-26559758) * f 3 * f 4+(-26559758) * f 3 * f 5+(81392) * f 4 * f 5+(-36676176) * f 1 * f 2 * f 3+($

$-31190040) * f 1 * f 2 * f 4+(20749160) * f 1 * f 2 * f 5+(20749160) * f 1 * f 3 * f 4+(-31190040)$

$* f 1 * f 3 * f 5+(-36676176) * f 1 * f 4 * f 5+(34599032) * f 2 * f 3 * f 4+(14338500) * f 2 * f 3 * f 5$

$+(-14341040) * f 2 * f 4 * f 5+(26393294) * f 3 * f 4 * f 5+(-7256176) * f 1 * f 2 * f 3 * f 4$

$+(36797216) * f 1 * f 2 * f 3 * f 5+(31073760) * f 1 * f 2 * f 4 * f 5+(-20868296) * f 1 * f 3 * f 4 * f 5$ 
$+(-34596492) * f 2 * f 3 * f 4 * f 5+(7370552) * f 1 * f 2 * f 3 * f 4 * f 5))))$

$3685276 h^{63}-17298246 h^{62}-10434148 h^{61}+13196647 h^{60}+15536880 h^{59}$

(113)

$-7170520 h^{58}-18338088 h^{57}+40696 h^{56}+18398608 h^{55}+7169250 h^{54}$

$-15595020 h^{53}-13279879 h^{52}+10374580 h^{51}+17299516 h^{50}-3628088 h^{49}$

$-18687492 h^{48}-3628088 h^{47}+17299516 h^{46}+10374580 h^{45}-13279879 h^{44}$

$-15595020 h^{43}+7169250 h^{42}+18398608 h^{41}+40696 h^{40}-18338088 h^{39}$

$-7170520 h^{38}+15536880 h^{37}+13196647 h^{36}-10434148 h^{35}-17298246 h^{34}$

$+3685276 h^{33}+18772564 h^{32}+3685276 h^{31}-17298246 h^{30}-10434148 h^{29}$

$+13196647 h^{28}+15536880 h^{27}-7170520 h^{26}-18338088 h^{25}+40696 h^{24}$

$+18398608 h^{23}+7169250 h^{22}-15595020 h^{21}-13279879 h^{20}+10374580 h^{19}$

$+17299516 h^{18}-3628088 h^{17}-18687492 h^{16}-3628088 h^{15}+17299516 h^{14}$

$+10374580 h^{13}-13279879 h^{12}-15595020 h^{11}+7169250 h^{10}+18398608 h^{9}$

$+40696 h^{8}-18338088 h^{7}-7170520 h^{6}+15536880 h^{5}+13196647 h^{4}-10434148 h^{3}$

$-17298246 h^{2}+3685276 h+18772565$

$u_{17}:=\operatorname{sort}\left(\operatorname{subs}\left(f 1=h, f 2=h^{2}, f 3=h^{4}, f 4=h^{8}, f 5=h^{16}, f 6=h^{32}\right.\right.$, expand $\left(1+(1+f 6) \cdot 2^{-1} \cdot((328)\right.$

$+(56) * f 1+(-76) * f 2+(-36) * f 3+(-208) * f 4+(88) * f 5+(-144) * f 1 * f 2+(280) * f 1$

$* f 3+(-140) * f 1 * f 4+(112) * f 1 * f 5+(82) * f 2 * f 3+(190) * f 2 * f 4+(-196) * f 2 * f 5$

$+(36) * f 3 * f 4+(36) * f 3 * f 5+(-208) * f 4 * f 5+(-28) * f 1 * f 2 * f 3+(84) * f 1 * f 2 * f 4+($

$-220) * f 1 * f 2 * f 5+(-220) * f 1 * f 3 * f 4+(84) * f 1 * f 3 * f 5+(-28) * f 1 * f 4 * f 5+(-196) * f 2$

$* f 3 * f 4+(190) * f 2 * f 3 * f 5+(82) * f 2 * f 4 * f 5+(-36) * f 3 * f 4 * f 5+(112) * f 1 * f 2 * f 3 * f 4$

$+(-140) * f 1 * f 2 * f 3 * f 5+(280) * f 1 * f 2 * f 4 * f 5+(-144) * f 1 * f 3 * f 4 * f 5+(-76) * f 2 * f 3$

$* f 4 * f 5+(56) * f 1 * f 2 * f 3 * f 4 * f 5))))$

$28 h^{63}-38 h^{62}-72 h^{61}-18 h^{60}+140 h^{59}+41 h^{58}-14 h^{57}-104 h^{56}-70 h^{55}$

(114)

$+95 h^{54}+42 h^{53}+18 h^{52}-110 h^{51}-98 h^{50}+56 h^{49}+44 h^{48}+56 h^{47}-98 h^{46}$

$-110 h^{45}+18 h^{44}+42 h^{43}+95 h^{42}-70 h^{41}-104 h^{40}-14 h^{39}+41 h^{38}+140 h^{37}$

$-18 h^{36}-72 h^{35}-38 h^{34}+28 h^{33}+164 h^{32}+28 h^{31}-38 h^{30}-72 h^{29}-18 h^{28}$

$+140 h^{27}+41 h^{26}-14 h^{25}-104 h^{24}-70 h^{23}+95 h^{22}+42 h^{21}+18 h^{20}-110 h^{19}$

$-98 h^{18}+56 h^{17}+44 h^{16}+56 h^{15}-98 h^{14}-110 h^{13}+18 h^{12}+42 h^{11}+95 h^{10}$

$-70 h^{9}-104 h^{8}-14 h^{7}+41 h^{6}+140 h^{5}-18 h^{4}-72 h^{3}-38 h^{2}+28 h+165$

$u_{18}:=\operatorname{sort}\left(\operatorname{subs}\left(f 1=h, f 2=h^{2}, f 3=h^{4}, f 4=h^{8}, f 5=h^{16}, f 6=h^{32}\right.\right.$, expand $\left(1+(1+f 6) \cdot 2^{-1} \cdot((20808)\right.$

$+(3836) * f 1+(-18894) * f 2+(14564) * f 3+(-208) * f 4+(-20392) * f 5+(-11528) * f 1$

$* f 2+(17212) * f 1 * f 3+(20384) * f 1 * f 4+(-4200) * f 1 * f 5+(-8020) * f 2 * f 3+(7748) * f 2$

$* f 4+(19166) * f 2 * f 5+(-14564) * f 3 * f 4+(-14564) * f 3 * f 5+(-208) * f 4 * f 5+(-20020)$

$* f 1 * f 2 * f 3+(-17044) * f 1 * f 2 * f 4+(11360) * f 1 * f 2 * f 5+(11360) * f 1 * f 3 * f 4+(-17044)$

$* f 1 * f 3 * f 5+(-20020) * f 1 * f 4 * f 5+(19166) * f 2 * f 3 * f 4+(7748) * f 2 * f 3 * f 5+(-8020) * f 2$

$* f 4 * f 5+(14564) * f 3 * f 4 * f 5+(-4200) * f 1 * f 2 * f 3 * f 4+(20384) * f 1 * f 2 * f 3 * f 5+(17212)$

$* f 1 * f 2 * f 4 * f 5+(-11528) * f 1 * f 3 * f 4 * f 5+(-18894) * f 2 * f 3 * f 4 * f 5+(3836) * f 1 * f 2 * f 3$ 
$* f 4 * f 5))))$

$1918 h^{63}-9447 h^{62}-5764 h^{61}+7282 h^{60}+8606 h^{59}-4010 h^{58}-10010 h^{57}-104 h^{56}$

$+10192 h^{55}+3874 h^{54}-8522 h^{53}-7282 h^{52}+5680 h^{51}+9583 h^{50}-2100 h^{49}$

$-10196 h^{48}-2100 h^{47}+9583 h^{46}+5680 h^{45}-7282 h^{44}-8522 h^{43}+3874 h^{42}$

$+10192 h^{41}-104 h^{40}-10010 h^{39}-4010 h^{38}+8606 h^{37}+7282 h^{36}-5764 h^{35}$

$-9447 h^{34}+1918 h^{33}+10404 h^{32}+1918 h^{31}-9447 h^{30}-5764 h^{29}+7282 h^{28}$

$+8606 h^{27}-4010 h^{26}-10010 h^{25}-104 h^{24}+10192 h^{23}+3874 h^{22}-8522 h^{21}$

$-7282 h^{20}+5680 h^{19}+9583 h^{18}-2100 h^{17}-10196 h^{16}-2100 h^{15}+9583 h^{14}$

$+5680 h^{13}-7282 h^{12}-8522 h^{11}+3874 h^{10}+10192 h^{9}-104 h^{8}-10010 h^{7}$

$-4010 h^{6}+8606 h^{5}+7282 h^{4}-5764 h^{3}-9447 h^{2}+1918 h+10405$

$u_{19}:=\operatorname{sort}\left(\operatorname{subs}\left(f 1=h, f 2=h^{2}, f 3=h^{4}, f 4=h^{8}, f 5=h^{16}, f 6=h^{32}\right.\right.$, expand $\left(1+(1+f 6) \cdot 2^{-1} \cdot((24)+(\right.$

$-14) * f 1+(-8) * f 2+(-16) * f 3+(-24) * f 5+(22) * f 1 * f 2+(-4) * f 1 * f 3+(18) * f 1 * f 4$

$+(14) * f 1 * f 5+(20) * f 2 * f 3+(-20) * f 2 * f 4+(8) * f 2 * f 5+(16) * f 3 * f 4+(16) * f 3 * f 5$

$+(-18) * f 1 * f 2 * f 3+(4) * f 1 * f 2 * f 4+(-22) * f 1 * f 2 * f 5+(-22) * f 1 * f 3 * f 4+(4) * f 1 * f 3$

$* f 5+(-18) * f 1 * f 4 * f 5+(8) * f 2 * f 3 * f 4+(-20) * f 2 * f 3 * f 5+(20) * f 2 * f 4 * f 5+(-16) * f 3$

$* f 4 * f 5+(14) * f 1 * f 2 * f 3 * f 4+(18) * f 1 * f 2 * f 3 * f 5+(-4) * f 1 * f 2 * f 4 * f 5+(22) * f 1 * f 3 * f 4$

$* f 5+(-8) * f 2 * f 3 * f 4 * f 5+(-14) * f 1 * f 2 * f 3 * f 4 * f 5))))$

$-7 h^{63}-4 h^{62}+11 h^{61}-8 h^{60}-2 h^{59}+10 h^{58}-9 h^{57}+9 h^{55}-10 h^{54}+2 h^{53}$

(116)

$+8 h^{52}-11 h^{51}+4 h^{50}+7 h^{49}-12 h^{48}+7 h^{47}+4 h^{46}-11 h^{45}+8 h^{44}+2 h^{43}$

$-10 h^{42}+9 h^{41}-9 h^{39}+10 h^{38}-2 h^{37}-8 h^{36}+11 h^{35}-4 h^{34}-7 h^{33}+12 h^{32}$

$-7 h^{31}-4 h^{30}+11 h^{29}-8 h^{28}-2 h^{27}+10 h^{26}-9 h^{25}+9 h^{23}-10 h^{22}+2 h^{21}$

$+8 h^{20}-11 h^{19}+4 h^{18}+7 h^{17}-12 h^{16}+7 h^{15}+4 h^{14}-11 h^{13}+8 h^{12}+2 h^{11}$

$-10 h^{10}+9 h^{9}-9 h^{7}+10 h^{6}-2 h^{5}-8 h^{4}+11 h^{3}-4 h^{2}-7 h+13$

$u_{20}:=\operatorname{sort}\left(\operatorname{subs}\left(f 1=h, f 2=h^{2}, f 3=h^{4}, f 4=h^{8}, f 5=h^{16}, f 6=h^{32}\right.\right.$, expand $\left(1+(1+f 6) \cdot 2^{-1} \cdot((24)\right.$

$+(4) * f 1+(-20) * f 2+(16) * f 3+(-24) * f 5+(-14) * f 1 * f 2+(18) * f 1 * f 3+(22) * f 1 * f 4$

$+(-4) * f 1 * f 5+(-8) * f 2 * f 3+(8) * f 2 * f 4+(20) * f 2 * f 5+(-16) * f 3 * f 4+(-16) * f 3 * f 5$

$+(-22) * f 1 * f 2 * f 3+(-18) * f 1 * f 2 * f 4+(14) * f 1 * f 2 * f 5+(14) * f 1 * f 3 * f 4+(-18) * f 1$

$* f 3 * f 5+(-22) * f 1 * f 4 * f 5+(20) * f 2 * f 3 * f 4+(8) * f 2 * f 3 * f 5+(-8) * f 2 * f 4 * f 5+(16)$

$* f 3 * f 4 * f 5+(-4) * f 1 * f 2 * f 3 * f 4+(22) * f 1 * f 2 * f 3 * f 5+(18) * f 1 * f 2 * f 4 * f 5+(-14) * f 1$

$* f 3 * f 4 * f 5+(-20) * f 2 * f 3 * f 4 * f 5+(4) * f 1 * f 2 * f 3 * f 4 * f 5))))$

$2 h^{63}-10 h^{62}-7 h^{61}+8 h^{60}+9 h^{59}-4 h^{58}-11 h^{57}+11 h^{55}+4 h^{54}-9 h^{53}-8 h^{52}$

(117)

$+7 h^{51}+10 h^{50}-2 h^{49}-12 h^{48}-2 h^{47}+10 h^{46}+7 h^{45}-8 h^{44}-9 h^{43}+4 h^{42}$

$+11 h^{41}-11 h^{39}-4 h^{38}+9 h^{37}+8 h^{36}-7 h^{35}-10 h^{34}+2 h^{33}+12 h^{32}+2 h^{31}$

$-10 h^{30}-7 h^{29}+8 h^{28}+9 h^{27}-4 h^{26}-11 h^{25}+11 h^{23}+4 h^{22}-9 h^{21}-8 h^{20}$

$+7 h^{19}+10 h^{18}-2 h^{17}-12 h^{16}-2 h^{15}+10 h^{14}+7 h^{13}-8 h^{12}-9 h^{11}+4 h^{10}$

$+11 h^{9}-11 h^{7}-4 h^{6}+9 h^{5}+8 h^{4}-7 h^{3}-10 h^{2}+2 h+13$

$u_{21}:=\operatorname{sort}\left(\operatorname{subs}\left(f 1=h, f 2=h^{2}, f 3=h^{4}, f 4=h^{8}, f 5=h^{16}, f 6=h^{32}\right.\right.$, expand $\left(1+(1+f 6) \cdot 2^{-1}((272)\right.$ 
$+(138) * f 1+(-108) * f 2+(-36) * f 3+(-208) * f 4+(144) * f 5+(-188) * f 1 * f 2+(130)$

$* f 1 * f 3+(-54) * f 1 * f 4+(30) * f 1 * f 5+(86) * f 2 * f 3+(186) * f 2 * f 4+(-164) * f 2 * f 5$

$+(36) * f 3 * f 4+(36) * f 3 * f 5+(-208) * f 4 * f 5+(-114) * f 1 * f 2 * f 3+(234) * f 1 * f 2 * f 4+($

$-176) * f 1 * f 2 * f 5+(-176) * f 1 * f 3 * f 4+(234) * f 1 * f 3 * f 5+(-114) * f 1 * f 4 * f 5+(-164)$

$* f 2 * f 3 * f 4+(186) * f 2 * f 3 * f 5+(86) * f 2 * f 4 * f 5+(-36) * f 3 * f 4 * f 5+(30) * f 1 * f 2 * f 3 * f 4$

$+(-54) * f 1 * f 2 * f 3 * f 5+(130) * f 1 * f 2 * f 4 * f 5+(-188) * f 1 * f 3 * f 4 * f 5+(-108) * f 2 * f 3$

$* f 4 * f 5+(138) * f 1 * f 2 * f 3 * f 4 * f 5))))$

$69 h^{63}-54 h^{62}-94 h^{61}-18 h^{60}+65 h^{59}+43 h^{58}-57 h^{57}-104 h^{56}-27 h^{55}+93 h^{54}$ (118)

$+117 h^{53}+18 h^{52}-88 h^{51}-82 h^{50}+15 h^{49}+72 h^{48}+15 h^{47}-82 h^{46}-88 h^{45}$

$+18 h^{44}+117 h^{43}+93 h^{42}-27 h^{41}-104 h^{40}-57 h^{39}+43 h^{38}+65 h^{37}-18 h^{36}$

$-94 h^{35}-54 h^{34}+69 h^{33}+136 h^{32}+69 h^{31}-54 h^{30}-94 h^{29}-18 h^{28}+65 h^{27}$

$+43 h^{26}-57 h^{25}-104 h^{24}-27 h^{23}+93 h^{22}+117 h^{21}+18 h^{20}-88 h^{19}-82 h^{18}$

$+15 h^{17}+72 h^{16}+15 h^{15}-82 h^{14}-88 h^{13}+18 h^{12}+117 h^{11}+93 h^{10}-27 h^{9}$

$-104 h^{8}-57 h^{7}+43 h^{6}+65 h^{5}-18 h^{4}-94 h^{3}-54 h^{2}+69 h+137$

$u_{22}:=\operatorname{sort}\left(\operatorname{subs}\left(f 1=h, f 2=h^{2}, f 3=h^{4}, f 4=h^{8}, f 5=h^{16}, f 6=h^{32}\right.\right.$, expand $\left(1+(1+f 6) \cdot 2^{-1} \cdot((1216)\right.$

$+(-772) * f 1+(-124) * f 2+(-276) * f 3+(-208) * f 4+(-800) * f 5+(612) * f 1 * f 2+($

$-486) * f 1 * f 3+(728) * f 1 * f 4+(604) * f 1 * f 5+(898) * f 2 * f 3+(-626) * f 2 * f 4+(-148) * f 2$

$* f 5+(276) * f 3 * f 4+(276) * f 3 * f 5+(-208) * f 4 * f 5+(-560) * f 1 * f 2 * f 3+(122) * f 1 * f 2$

$* f 4+(-248) * f 1 * f 2 * f 5+(-248) * f 1 * f 3 * f 4+(122) * f 1 * f 3 * f 5+(-560) * f 1 * f 4 * f 5+($

$-148) * f 2 * f 3 * f 4+(-626) * f 2 * f 3 * f 5+(898) * f 2 * f 4 * f 5+(-276) * f 3 * f 4 * f 5+(604) * f 1$

$* f 2 * f 3 * f 4+(728) * f 1 * f 2 * f 3 * f 5+(-486) * f 1 * f 2 * f 4 * f 5+(612) * f 1 * f 3 * f 4 * f 5+(-124)$

$* f 2 * f 3 * f 4 * f 5+(-772) * f 1 * f 2 * f 3 * f 4 * f 5))))$

$-386 h^{63}-62 h^{62}+306 h^{61}-138 h^{60}-243 h^{59}+449 h^{58}-280 h^{57}-104 h^{56}$

(119)

$+364 h^{55}-313 h^{54}+61 h^{53}+138 h^{52}-124 h^{51}-74 h^{50}+302 h^{49}-400 h^{48}$

$+302 h^{47}-74 h^{46}-124 h^{45}+138 h^{44}+61 h^{43}-313 h^{42}+364 h^{41}-104 h^{40}$

$-280 h^{39}+449 h^{38}-243 h^{37}-138 h^{36}+306 h^{35}-62 h^{34}-386 h^{33}+608 h^{32}$

$-386 h^{31}-62 h^{30}+306 h^{29}-138 h^{28}-243 h^{27}+449 h^{26}-280 h^{25}-104 h^{24}$

$+364 h^{23}-313 h^{22}+61 h^{21}+138 h^{20}-124 h^{19}-74 h^{18}+302 h^{17}-400 h^{16}$

$+302 h^{15}-74 h^{14}-124 h^{13}+138 h^{12}+61 h^{11}-313 h^{10}+364 h^{9}-104 h^{8}$

$-280 h^{7}+449 h^{6}-243 h^{5}-138 h^{4}+306 h^{3}-62 h^{2}-386 h+609$

$u_{23}:=\operatorname{sort}\left(\operatorname{subs}\left(f l=h, f 2=h^{2}, f 3=h^{4}, f 4=h^{8}, f 5=h^{16}, f 6=h^{32}\right.\right.$, expand $\left(1+(1+f 6) \cdot 2^{-1} \cdot((352)\right.$

$+(-148) * f 1+(-184) * f 2+(-70) * f 3+(-204) * f 4+(344) * f 5+(304) * f 1 * f 2+(-200)$

$* f 1 * f 3+(46) * f 1 * f 4+(-150) * f 1 * f 5+(188) * f 2 * f 3+(186) * f 2 * f 4+(-190) * f 2 * f 5$

$+(-74) * f 3 * f 4+(-74) * f 3 * f 5+(-204) * f 4 * f 5+(48) * f 1 * f 2 * f 3+(-202) * f 1 * f 2 * f 4$

$+(302) * f l * f 2 * f 5+(302) * f 1 * f 3 * f 4+(-202) * f l * f 3 * f 5+(48) * f 1 * f 4 * f 5+(-190) * f 2$

$* f 3 * f 4+(186) * f 2 * f 3 * f 5+(188) * f 2 * f 4 * f 5+(-70) * f 3 * f 4 * f 5+(-150) * f 1 * f 2 * f 3 * f 4$

$+(46) * f 1 * f 2 * f 3 * f 5+(-200) * f 1 * f 2 * f 4 * f 5+(304) * f 1 * f 3 * f 4 * f 5+(-184) * f 2 * f 3 * f 4$

$* f 5+(-148) * f 1 * f 2 * f 3 * f 4 * f 5))))$

$-74 h^{63}-92 h^{62}+152 h^{61}-35 h^{60}-100 h^{59}+94 h^{58}+24 h^{57}-102 h^{56}+23 h^{55}$

(120) 


$$
\begin{aligned}
& +93 h^{54}-101 h^{53}-37 h^{52}+151 h^{51}-95 h^{50}-75 h^{49}+172 h^{48}-75 h^{47}-95 h^{46} \\
& +151 h^{45}-37 h^{44}-101 h^{43}+93 h^{42}+23 h^{41}-102 h^{40}+24 h^{39}+94 h^{38} \\
& -100 h^{37}-35 h^{36}+152 h^{35}-92 h^{34}-74 h^{33}+176 h^{32}-74 h^{31}-92 h^{30} \\
& +152 h^{29}-35 h^{28}-100 h^{27}+94 h^{26}+24 h^{25}-102 h^{24}+23 h^{23}+93 h^{22} \\
& -101 h^{21}-37 h^{20}+151 h^{19}-95 h^{18}-75 h^{17}+172 h^{16}-75 h^{15}-95 h^{14} \\
& +151 h^{13}-37 h^{12}-101 h^{11}+93 h^{10}+23 h^{9}-102 h^{8}+24 h^{7}+94 h^{6}-100 h^{5} \\
& -35 h^{4}+152 h^{3}-92 h^{2}-74 h+177 \\
& u_{24}:=\operatorname{sort}\left(\operatorname { s u b s } \left(f l = h , f 2 = h ^ { 2 } , f 3 = h ^ { 4 } , f 4 = h ^ { 8 } , f 5 = h ^ { 1 6 } , f 6 = h ^ { 3 2 } \text { , expand } \left(1+(1+f 6) \cdot 2^{-1} \cdot((11424)\right.\right.\right. \\
& +(4402) * f 1+(-8004) * f 2+(-64) * f 3+(-11428) * f 4+(11432) * f 5+(-10512) * f 1 * f 2 \\
& +(10410) * f 1 * f 3+(-4432) * f 1 * f 4+(4442) * f 1 * f 5+(7952) * f 2 * f 3+(7956) * f 2 * f 4+( \\
& -7904) * f 2 * f 5+(64) * f 3 * f 4+(64) * f 3 * f 5+(-11428) * f 4 * f 5+(-4412) * f 1 * f 2 * f 3 \\
& +(10472) * f 1 * f 2 * f 4+(-10370) * f 1 * f 2 * f 5+(-10370) * f 1 * f 3 * f 4+(10472) * f 1 * f 3 * f 5 \\
& +(-4412) * f 1 * f 4 * f 5+(-7904) * f 2 * f 3 * f 4+(7956) * f 2 * f 3 * f 5+(7952) * f 2 * f 4 * f 5+( \\
& -64) * f 3 * f 4 * f 5+(4442) * f 1 * f 2 * f 3 * f 4+(-4432) * f 1 * f 2 * f 3 * f 5+(10410) * f 1 * f 2 * f 4 * f 5 \\
& +(-10512) * f 1 * f 3 * f 4 * f 5+(-8004) * f 2 * f 3 * f 4 * f 5+(4402) * f 1 * f 2 * f 3 * f 4 * f 5)))) \\
& 2201 h^{63}-4002 h^{62}-5256 h^{61}-32 h^{60}+5205 h^{59}+3976 h^{58}-2206 h^{57}-5714 h^{56} \\
& -2216 h^{55}+3978 h^{54}+5236 h^{53}+32 h^{52}-5185 h^{51}-3952 h^{50}+2221 h^{49} \\
& +5716 h^{48}+2221 h^{47}-3952 h^{46}-5185 h^{45}+32 h^{44}+5236 h^{43}+3978 h^{42} \\
& -2216 h^{41}-5714 h^{40}-2206 h^{39}+3976 h^{38}+5205 h^{37}-32 h^{36}-5256 h^{35} \\
& -4002 h^{34}+2201 h^{33}+5712 h^{32}+2201 h^{31}-4002 h^{30}-5256 h^{29}-32 h^{28} \\
& +5205 h^{27}+3976 h^{26}-2206 h^{25}-5714 h^{24}-2216 h^{23}+3978 h^{22}+5236 h^{21} \\
& +32 h^{20}-5185 h^{19}-3952 h^{18}+2221 h^{17}+5716 h^{16}+2221 h^{15}-3952 h^{14} \\
& -5185 h^{13}+32 h^{12}+5236 h^{11}+3978 h^{10}-2216 h^{9}-5714 h^{8}-2206 h^{7}+3976 h^{6} \\
& +5205 h^{5}-32 h^{4}-5256 h^{3}-4002 h^{2}+2201 h+5713 \\
& u_{25}:=\operatorname{sort}\left(\operatorname { s u b s } \left(f 1 = h , f 2 = h ^ { 2 } , f 3 = h ^ { 4 } , f 4 = h ^ { 8 } , f 5 = h ^ { 1 6 } , f 6 = h ^ { 3 2 } \text { , expand } \left(1+(1+f 6) \cdot 2^{-1} \cdot((-5520)\right.\right.\right. \\
& +(-3614) * f 1+(1052) * f 2+(10062) * f 3+(-9112) * f 4+(24032) * f 5+(6398) * f 1 * f 2 \\
& +(10524) * f 1 * f 3+(-16642) * f 1 * f 4+(20684) * f 1 * f 5+(6946) * f 2 * f 3+(-19934) * f 2 \\
& * f 4+(11936) * f 2 * f 5+(-10206) * f 3 * f 4+(-10206) * f 3 * f 5+(-9112) * f 4 * f 5+(-224) \\
& * f 1 * f 2 * f 3+(-17654) * f 1 * f 2 * f 4+(528) * f 1 * f 2 * f 5+(528) * f 1 * f 3 * f 4+(-17654) * f 1 \\
& * f 3 * f 5+(-224) * f 1 * f 4 * f 5+(11936) * f 2 * f 3 * f 4+(-19934) * f 2 * f 3 * f 5+(6946) * f 2 * f 4 \\
& * f 5+(10062) * f 3 * f 4 * f 5+(20684) * f 1 * f 2 * f 3 * f 4+(-16642) * f 1 * f 2 * f 3 * f 5+(10524) \\
& * f 1 * f 2 * f 4 * f 5+(6398) * f 1 * f 3 * f 4 * f 5+(1052) * f 2 * f 3 * f 4 * f 5+(-3614) * f 1 * f 2 * f 3 * f 4 \\
& * f 5))) \text { ) } \\
& -1807 h^{63}+526 h^{62}+3199 h^{61}+5031 h^{60}+5262 h^{59}+3473 h^{58}-112 h^{57}-4556 h^{56} \\
& -8321 h^{55}-9967 h^{54}-8827 h^{53}-5103 h^{52}+264 h^{51}+5968 h^{50}+10342 h^{49} \\
& +12016 h^{48}+10342 h^{47}+5968 h^{46}+264 h^{45}-5103 h^{44}-8827 h^{43}-9967 h^{42} \\
& -8321 h^{41}-4556 h^{40}-112 h^{39}+3473 h^{38}+5262 h^{37}+5031 h^{36}+3199 h^{35}
\end{aligned}
$$




$$
\begin{aligned}
& +526 h^{34}-1807 h^{33}-2760 h^{32}-1807 h^{31}+526 h^{30}+3199 h^{29}+5031 h^{28} \\
& +5262 h^{27}+3473 h^{26}-112 h^{25}-4556 h^{24}-8321 h^{23}-9967 h^{22}-8827 h^{21} \\
& -5103 h^{20}+264 h^{19}+5968 h^{18}+10342 h^{17}+12016 h^{16}+10342 h^{15}+5968 h^{14} \\
& +264 h^{13}-5103 h^{12}-8827 h^{11}-9967 h^{10}-8321 h^{9}-4556 h^{8}-112 h^{7}+3473 h^{6} \\
& +5262 h^{5}+5031 h^{4}+3199 h^{3}+526 h^{2}-1807 h-2759 \\
& u_{26}:=\operatorname{sort}\left(\operatorname { s u b s } \left(f 1 = h , f 2 = h ^ { 2 } , f 3 = h ^ { 4 } , f 4 = h ^ { 8 } , f 5 = h ^ { 1 6 } , f 6 = h ^ { 3 2 } \text { , expand } \left(1+(1+f 6) \cdot 2^{-1} \cdot((4) * f 1\right.\right.\right. \\
& +(-2) * f 2+(-2) * f 3+(4) * f 4+(-2) * f 1 * f 2+(-2) * f 1 * f 3+(2) * f 1 * f 5+(2) * f 2 * f 3 \\
& +(-2) * f 3 * f 4+(-2) * f 3 * f 5+(4) * f 4 * f 5+(-2) * f 1 * f 2 * f 4+(-2) * f 1 * f 3 * f 5+(2) * f 2 \\
& * f 4 * f 5+(-2) * f 3 * f 4 * f 5+(2) * f 1 * f 2 * f 3 * f 4+(-2) * f 1 * f 2 * f 4 * f 5+(-2) * f 1 * f 3 * f 4 * f 5 \\
& +(-2) * f 2 * f 3 * f 4 * f 5+(4) * f 1 * f 2 * f 3 * f 4 * f 5)))) \\
& 2 h^{63}-h^{62}-h^{61}-h^{60}-h^{59}+h^{58}+2 h^{56}-h^{53}-h^{52}+h^{49}+h^{47}-h^{44}-h^{43} \\
& +2 h^{40}+h^{38}-h^{37}-h^{36}-h^{35}-h^{34}+2 h^{33}+2 h^{31}-h^{30}-h^{29}-h^{28}-h^{27} \\
& +h^{26}+2 h^{24}-h^{21}-h^{20}+h^{17}+h^{15}-h^{12}-h^{11}+2 h^{8}+h^{6}-h^{5}-h^{4}-h^{3}-h^{2} \\
& +2 h+1
\end{aligned}
$$

Vamos agora aplicar $f_{1}$ em cada um $\operatorname{dos} u_{i}, i=1,2, \ldots 26$ e obtemos $v_{i}$ :

$$
\begin{aligned}
v_{1}:= & \operatorname{sort}\left(\text { expand }\left(\text { subs }\left(h=x+1, u_{1}\right)\right) \bmod 2\right) \\
x^{62} & +x^{61}+x^{60}+x^{56}+x^{55}+x^{54}+x^{50}+x^{49}+x^{48}+x^{44}+x^{43}+x^{42}+x^{38}+x^{37}+x^{36} \\
& +x^{32}+x^{31}+x^{30}+x^{26}+x^{25}+x^{24}+x^{20}+x^{19}+x^{18}+x^{14}+x^{13}+x^{12}+x^{8}+x^{7} \\
& +x^{6}+1 \\
v_{2}:= & \operatorname{sort}\left(\text { expand }\left(\operatorname{subs}\left(h=x+1, u_{2}\right)\right) \bmod 2\right) \\
x^{62} & +x^{61}+x^{59}+x^{58}+x^{56}+x^{55}+x^{54}+x^{52}+x^{51}+x^{49}+x^{48}+x^{38}+x^{37}+x^{35}+x^{34} \\
& +x^{32}+x^{31}+x^{30}+x^{28}+x^{27}+x^{25}+x^{24}+x^{14}+x^{13}+x^{11}+x^{10}+1 \\
v_{3}:= & \operatorname{sort}\left(\text { expand }\left(\operatorname{subs}^{30}\left(h=x+1, u_{3}\right)\right)^{\bmod 2}\right) \\
x^{62} & +x^{61}+x^{59}+x^{58}+x^{56}+x^{55}+x^{53}+x^{52}+x^{50}+x^{49}+x^{47}+x^{46}+x^{44}+x^{43}+x^{41} \\
& +x^{40}+x^{38}+x^{37}+x^{36}+x^{32}+x^{31}+x^{30}+x^{28}+x^{27}+x^{26}+x^{24}+x^{23}+x^{22}+x^{20} \\
& +x^{19}+x^{18}+x^{16}+x^{15}+x^{14}+x^{12}+x^{11}+x^{10}+x^{8}+x^{7}+x^{6}+1
\end{aligned}
$$$$
v_{4}:=\operatorname{sort}\left(\operatorname{expand}\left(\operatorname{subs}\left(h=x+1, u_{4}\right)\right) \bmod 2\right)
$$$$
x^{63}+x^{62}+x^{60}+x^{59}+x^{57}+x^{56}+x^{54}+x^{53}+x^{51}+x^{50}+x^{48}+x^{47}+x^{46}+x^{44}+x^{43}
$$$$
+x^{41}+x^{40}+x^{38}+x^{37}+x^{35}+x^{34}+x^{32}+x^{31}+x^{30}+x^{28}+x^{27}+x^{25}+x^{24}+x^{22}
$$$$
+x^{21}+x^{19}+x^{18}+1
$$$$
v_{5}:=\operatorname{sort}\left(\text { expand }\left(\operatorname{subs}\left(h=x+1, u_{5}\right)\right) \bmod 2\right)
$$$$
x^{62}+x^{61}+x^{59}+x^{58}+x^{56}+x^{55}+x^{53}+x^{52}+x^{46}+x^{45}+x^{43}+x^{42}+x^{40}+x^{39}+x^{37}
$$$$
+x^{36}+x^{30}+x^{29}+x^{27}+x^{26}+x^{24}+x^{23}+x^{21}+x^{20}+x^{14}+x^{13}+x^{12}+x^{8}+x^{7}
$$ 


$$
\begin{aligned}
& +x^{6}+1 \\
& v_{6}:=\operatorname{sort}\left(\text { expand }\left(\operatorname{subs}\left(h=x+1, u_{6}\right)\right) \bmod 2\right) \\
& x^{62}+x^{61}+x^{59}+x^{58}+x^{56}+x^{55}+x^{53}+x^{52}+x^{50}+x^{49}+x^{47}+x^{46}+x^{44}+x^{43}+x^{41} \\
& +x^{40}+x^{30}+x^{29}+x^{27}+x^{26}+x^{22}+x^{21}+x^{19}+x^{18}+x^{14}+x^{13}+x^{11}+x^{10}+1 \\
& v_{7}:=\operatorname{sort}\left(\operatorname{expand}\left(\operatorname{subs}\left(h=x+1, u_{7}\right)\right) \bmod 2\right) \\
& x^{63}+x^{62}+x^{60}+x^{59}+x^{57}+x^{56}+x^{54}+x^{53}+x^{52}+x^{48}+x^{47}+x^{46}+x^{44}+x^{43}+x^{41} \\
& +x^{40}+x^{38}+x^{37}+x^{36}+x^{32}+x^{31}+x^{30}+x^{28}+x^{27}+x^{25}+x^{24}+x^{22}+x^{21}+x^{20} \\
& +x^{16}+x^{15}+x^{14}+x^{12}+x^{11}+x^{10}+x^{8}+x^{7}+x^{6}+1 \\
& v_{8}:=\operatorname{sort}\left(\text { expand }\left(\operatorname{subs}\left(h=x+1, u_{8}\right)\right) \bmod 2\right) \\
& x^{62}+x^{61}+x^{59}+x^{58}+x^{56}+x^{55}+x^{53}+x^{52}+x^{50}+x^{49}+x^{47}+x^{46}+x^{44}+x^{43}+x^{41} \\
& +x^{40}+x^{38}+x^{37}+x^{35}+x^{34}+1 \\
& v_{9}:=\operatorname{sort}\left(\operatorname{expand}\left(\operatorname{subs}\left(h=x+1, u_{9}\right)\right) \bmod 2\right) \\
& x^{63}+x^{62}+x^{60}+x^{59}+x^{57}+x^{56}+x^{54}+x^{53}+x^{51}+x^{50}+x^{48}+x^{47}+x^{45}+x^{44}+x^{42} \\
& +x^{41}+x^{39}+x^{38}+x^{36}+x^{35}+x^{34}+x^{32}+x^{31}+x^{30}+x^{26}+x^{25}+x^{24}+x^{20}+x^{19} \\
& +x^{18}+x^{14}+x^{13}+x^{12}+x^{8}+x^{7}+x^{6}+1 \\
& v_{10}:=\operatorname{sort}\left(\text { expand }\left(\operatorname{subs}\left(h=x+1, u_{10}\right)\right) \bmod 2\right) \\
& x^{63}+x^{62}+x^{60}+x^{59}+x^{57}+x^{56}+x^{54}+x^{53}+x^{51}+x^{50}+x^{48}+x^{47}+x^{45}+x^{44}+x^{42} \\
& +x^{41}+x^{40}+x^{32}+x^{31}+x^{30}+x^{28}+x^{27}+x^{25}+x^{24}+x^{14}+x^{13}+x^{11}+x^{10}+1 \\
& v_{11}:=\operatorname{sort}\left(\operatorname{expand}\left(\operatorname{subs}\left(h=x+1, u_{11}\right)\right) \bmod 2\right) \\
& x^{63}+x^{62}+x^{60}+x^{59}+x^{58}+x^{56}+x^{55}+x^{54}+x^{52}+x^{51}+x^{50}+x^{48}+x^{47}+x^{46}+x^{44} \\
& +x^{43}+x^{42}+x^{40}+x^{39}+x^{38}+x^{36}+x^{35}+x^{34}+x^{32}+x^{31}+x^{30}+x^{28}+x^{27}+x^{26} \\
& +x^{24}+x^{23}+x^{22}+x^{20}+x^{19}+x^{18}+x^{16}+x^{15}+x^{14}+x^{12}+x^{11}+x^{10}+x^{8}+x^{7} \\
& +x^{6}+1
\end{aligned}
$$$$
v_{12}:=\operatorname{sort}\left(\operatorname{expand}\left(\operatorname{subs}\left(h=x+1, u_{12}\right)\right) \bmod 2\right)
$$$$
x^{62}+x^{61}+x^{59}+x^{58}+x^{56}+x^{55}+x^{53}+x^{52}+x^{50}+x^{49}+x^{48}+x^{32}+x^{31}+x^{30}+x^{28}
$$$$
+x^{27}+x^{25}+x^{24}+x^{22}+x^{21}+x^{19}+x^{18}+1
$$

$v_{13}:=\operatorname{sort}\left(\right.$ expand $\left.\left(\operatorname{subs}\left(h=x+1, u_{13}\right)\right) \bmod 2\right)$

$$
\begin{aligned}
x^{62} & +x^{61}+x^{59}+x^{58}+x^{56}+x^{55}+x^{53}+x^{52}+x^{50}+x^{49}+x^{47}+x^{46}+x^{42}+x^{41}+x^{40} \\
& +x^{36}+x^{35}+x^{34}+x^{30}+x^{29}+x^{27}+x^{26}+x^{24}+x^{23}+x^{21}+x^{20}+x^{14}+x^{13}+x^{12} \\
& +x^{8}+x^{7}+x^{6}+1
\end{aligned}
$$$$
v_{14}:=\operatorname{sort}\left(\text { expand }\left(\operatorname{subs}\left(h=x+1, u_{14}\right)\right) \bmod 2\right)
$$$$
x^{62}+x^{61}+x^{59}+x^{58}+x^{54}+x^{53}+x^{51}+x^{50}+x^{46}+x^{45}+x^{43}+x^{42}+x^{38}+x^{37}+x^{35}
$$ 


$$
\begin{aligned}
& +x^{34}+x^{30}+x^{29}+x^{27}+x^{26}+x^{22}+x^{21}+x^{19}+x^{18}+x^{14}+x^{13}+x^{11}+x^{10}+1 \\
& v_{15}:=\operatorname{sort}\left(\text { expand }\left(\operatorname{subs}\left(h=x+1, u_{15}\right)\right) \bmod 2\right) \\
& x^{62}+x^{61}+x^{59}+x^{58}+x^{56}+x^{55}+x^{53}+x^{52}+x^{50}+x^{49}+x^{47}+x^{46}+x^{44}+x^{43}+x^{42} \\
& +x^{40}+x^{39}+x^{38}+x^{36}+x^{35}+x^{34}+x^{32}+x^{31}+x^{30}+x^{28}+x^{27}+x^{25}+x^{24}+x^{22} \\
& +x^{21}+x^{20}+x^{16}+x^{15}+x^{14}+x^{12}+x^{11}+x^{10}+x^{8}+x^{7}+x^{6}+1 \\
& v_{16}:=\operatorname{sort}\left(\operatorname{expand}\left(\operatorname{subs}\left(h=x+1, u_{16}\right)\right) \bmod 2\right) \\
& x^{60}+x^{56}+1 \\
& v_{17}:=\operatorname{sort}\left(\operatorname{expand}\left(\operatorname{subs}\left(h=x+1, u_{17}\right)\right) \bmod 2\right) \\
& x^{58}+x^{56}+x^{54}+x^{52}+1 \\
& v_{18}:=\operatorname{sort}\left(\text { expand }\left(\operatorname{subs}\left(h=x+1, u_{18}\right)\right) \bmod 2\right) \\
& x^{62}+x^{60}+x^{58}+x^{56}+x^{54}+x^{52}+1 \\
& v_{19}:=\operatorname{sort}\left(\operatorname{expand}\left(\operatorname{subs}\left(h=x+1, u_{19}\right)\right) \bmod 2\right) \\
& x^{63}+x^{62}+x^{59}+x^{58}+x^{57}+x^{56}+x^{53}+x^{52}+x^{51}+x^{50}+1 \\
& v_{20}:=\operatorname{sort}\left(\text { expand }\left(\operatorname{subs}\left(h=x+1, u_{20}\right)\right) \bmod 2\right) \\
& x^{61}+x^{60}+x^{59}+x^{58}+x^{57}+x^{56}+x^{55}+x^{54}+x^{53}+x^{52}+x^{51}+x^{50}+1 \\
& v_{21}:=\operatorname{sort}\left(\operatorname{expand}\left(\operatorname{subs}\left(h=x+1, u_{21}\right)\right) \bmod 2\right) \\
& x^{63}+x^{62}+x^{61}+x^{60}+x^{58}+x^{57}+x^{54}+x^{53}+x^{51}+x^{50}+1 \\
& v_{22}:=\operatorname{sort}\left(\text { expand }\left(\operatorname{subs}\left(h=x+1, u_{22}\right)\right) \bmod 2\right) \\
& x^{59}+x^{57}+x^{54}+x^{53}+x^{51}+x^{50}+1 \\
& x^{60}+x^{56}+x^{55}+x^{52}+x^{47}+x^{44}+1 \\
& x^{63}+x^{62}+x^{61}+x^{60}+x^{55}+x^{54}+x^{53}+x^{52}+x^{51}+x^{50}+x^{45}+x^{44}+x^{43}+x^{42}+1 \\
& v_{25}:=\operatorname{sort}\left(\text { expand }\left(\operatorname{subs}\left(h=x+1, u_{25}\right)\right) \bmod 2\right) \\
& x^{63}+x^{62}+x^{60}+x^{59}+x^{54}+x^{52}+x^{47}+x^{46}+1 \\
& v_{26}:=\operatorname{sort}\left(\text { expand }\left(\operatorname{subs}\left(h=x+1, u_{26}\right)\right) \bmod 2\right) \\
& x^{62}+x^{61}+x^{60}+x^{59}+x^{58}+x^{56}+x^{54}+x^{52}+x^{51}+x^{50}+x^{47}+x^{44}+x^{43}+x^{42}+x^{39} \\
& +x^{38}+1
\end{aligned}
$$

Vamos agora encontrar um conjunto multiplicatvamente independente que gera $\operatorname{Im}\left(j_{1}\right)=\left\langle h, v_{1}, v_{2}, \ldots, v_{26}\right\rangle$.

$$
\begin{aligned}
& e_{1}:=v_{1} \\
& x^{62}+x^{61}+x^{60}+x^{56}+x^{55}+x^{54}+x^{50}+x^{49}+x^{48}+x^{44}+x^{43}+x^{42}+x^{38}+x^{37}+x^{36}
\end{aligned}
$$




$$
\begin{aligned}
& +x^{32}+x^{31}+x^{30}+x^{26}+x^{25}+x^{24}+x^{20}+x^{19}+x^{18}+x^{14}+x^{13}+x^{12}+x^{8}+x^{7} \\
& +x^{6}+1 \\
& e_{2}:=v_{2} \\
& x^{62}+x^{61}+x^{59}+x^{58}+x^{56}+x^{55}+x^{54}+x^{52}+x^{51}+x^{49}+x^{48}+x^{38}+x^{37}+x^{35}+x^{34} \\
& +x^{32}+x^{31}+x^{30}+x^{28}+x^{27}+x^{25}+x^{24}+x^{14}+x^{13}+x^{11}+x^{10}+1 \\
& e_{3}:=\operatorname{sort}\left(\text { mtaylor }\left(\text { expand }\left(v_{3} \cdot e_{1}^{3} \cdot e_{2}\right), x, 64\right) \bmod 2\right) \\
& x^{63}+x^{61}+x^{55}+x^{54}+x^{53}+x^{48}+x^{47}+x^{46}+x^{39}+x^{38}+x^{31}+x^{30}+x^{29}+x^{24}+x^{15} \\
& +x^{14}+1 \\
& e_{4}:=v_{4} \\
& x^{63}+x^{62}+x^{60}+x^{59}+x^{57}+x^{56}+x^{54}+x^{53}+x^{51}+x^{50}+x^{48}+x^{47}+x^{46}+x^{44}+x^{43} \\
& +x^{41}+x^{40}+x^{38}+x^{37}+x^{35}+x^{34}+x^{32}+x^{31}+x^{30}+x^{28}+x^{27}+x^{25}+x^{24}+x^{22} \\
& +x^{21}+x^{19}+x^{18}+1 \\
& e_{5}:=\operatorname{sort}\left(\text { mtaylor }\left(\text { expand }\left(v_{5} \cdot e_{1}^{3} \cdot e_{4}\right), x, 64\right) \bmod 2\right) \\
& x^{60}+x^{58}+x^{56}+x^{55}+x^{54}+x^{51}+x^{48}+x^{47}+x^{45}+x^{44}+x^{43}+x^{38}+x^{36}+x^{32}+x^{31} \\
& +x^{29}+x^{28}+x^{24}+x^{23}+x^{22}+1 \\
& e_{6}:=\operatorname{sort}\left(\text { mtaylor }\left(\text { expand }\left(v_{6} \cdot e_{2}^{3} \cdot e_{4}\right), x, 64\right) \bmod 2\right) \\
& x^{63}+x^{62}+x^{58}+x^{55}+x^{54}+x^{53}+x^{52}+x^{48}+x^{46}+x^{44}+x^{43}+x^{42}+x^{39}+x^{38}+x^{29} \\
& +x^{28}+x^{27}+x^{26}+1 \\
& e_{7}:=\operatorname{sort}\left(\text { mtaylor }\left(\text { expand }\left(v_{7} \cdot e_{1}^{7} \cdot e_{2} \cdot e_{3}^{3} \cdot e_{4} \cdot e_{5} \cdot e_{6}\right), x, 64\right) \bmod 2\right) \\
& x^{63}+x^{61}+x^{60}+x^{54}+x^{52}+x^{50}+x^{42}+x^{40}+x^{38}+x^{36}+x^{31}+x^{30}+1 \\
& e_{8}:=\operatorname{sort}\left(\text { mtaylor }\left(\text { expand }\left(v_{8}\right), x, 64\right) \bmod 2\right) \\
& x^{62}+x^{61}+x^{59}+x^{58}+x^{56}+x^{55}+x^{53}+x^{52}+x^{50}+x^{49}+x^{47}+x^{46}+x^{44}+x^{43}+x^{41} \\
& +x^{40}+x^{38}+x^{37}+x^{35}+x^{34}+1 \\
& e_{9}:=\operatorname{sort}\left(\text { mtaylor }\left(\text { expand }\left(v_{9} \cdot e_{1}^{7} \cdot e_{8}\right), x, 64\right) \bmod 2\right) \\
& x^{63}+x^{59}+x^{56}+x^{55}+x^{54}+x^{53}+x^{52}+x^{48}+x^{47}+x^{45}+x^{39}+x^{38}+1 \\
& e_{10}:=\operatorname{sort}\left(\text { mtaylor }\left(\text { expand }\left(v_{10} \cdot e_{2}^{7} \cdot e_{8}\right), x, 64\right) \bmod 2\right) \\
& x^{58}+x^{57}+x^{55}+x^{52}+x^{45}+x^{44}+x^{43}+x^{42}+1 \\
& e_{11}:=\operatorname{sort}\left(\text { mtaylor }\left(\text { expand }\left(v_{11} \cdot e_{1}^{3} \cdot e_{2} \cdot e_{3}^{3} \cdot e_{8} \cdot e_{9} \cdot e_{10}\right), x, 64\right) \bmod 2\right) \\
& x^{63}+x^{61}+x^{60}+x^{48}+x^{47}+x^{46}+1 \\
& e_{12}:=\operatorname{sort}\left(\text { mtaylor }\left(\text { expand }\left(v_{12} \cdot e_{4}^{3} \cdot e_{8}\right), x, 64\right) \bmod 2\right) \\
& x^{63}+x^{62}+x^{59}+x^{58}+x^{57}+x^{56}+x^{53}+x^{52}+x^{51}+x^{50}+1 \\
& e_{13}:=\operatorname{sort}\left(\text { mtaylor }\left(\text { expand }\left(v_{13} \cdot e_{1}^{11} \cdot e_{4} \cdot e_{5}^{3} \cdot e_{8} \cdot e_{9} \cdot e_{12}\right), x, 64\right) \bmod 2\right) \\
& x^{63}+x^{62}+x^{61}+x^{60}+x^{55}+x^{54}+1
\end{aligned}
$$




$$
\begin{aligned}
& e_{14}:=\operatorname{sort}\left(\text { mtaylor }\left(\text { expand }\left(v_{14} \cdot e_{2}^{3} \cdot e_{4} \cdot e_{6}^{3} \cdot e_{8} \cdot e_{10} \cdot e_{12}\right), x, 64\right) \bmod 2\right) \\
& x^{61}+x^{60}+x^{59}+x^{58}+1 \\
& e_{15}:=\operatorname{sort}\left(\text { mtaylor }\left(\text { expand }\left(v_{15} \cdot e_{1}^{7} \cdot e_{2} \cdot e_{3}^{7} \cdot e_{4} \cdot e_{5} \cdot e_{6} \cdot e_{7}^{3} \cdot e_{8} \cdot e_{9} \cdot e_{10} \cdot e_{11} \cdot e_{12} \cdot e_{13} \cdot e_{14}\right), x, 64\right) \bmod 2\right) \\
& x^{63}+x^{62}+1
\end{aligned}
$$

$\operatorname{sort}\left(\right.$ mtaylor $\left.\left(\operatorname{expand}\left(v_{16} \cdot e_{3}^{4}\right), x, 64\right) \bmod 2\right) \#=v_{1}^{12} v_{2}^{4} v_{3}^{4} v_{16}=v_{1}^{-4} v_{2}^{4} v_{3}^{4} v_{16}$

$\operatorname{sort}\left(\right.$ mtaylor $\left.\left(\operatorname{expand}\left(v_{17} \cdot e_{6}^{2}\right), x, 64\right) \bmod 2\right) \#=v_{2}^{6} v_{4}^{2} v_{6}^{2} v_{17}=v_{2}^{-2} v_{4}^{2} v_{6}^{2} v_{17}$

$\operatorname{sort}\left(\operatorname{mtaylor}\left(\operatorname{expand}\left(v_{18} \cdot e_{6}^{2} \cdot e_{7}^{2}\right), x, 64\right) \bmod 2\right) \#=v_{1}^{6} v_{2}^{4} v_{3}^{6} v_{5}^{2} v_{6}^{4} v_{7}^{2} v_{18}$

$\operatorname{sort}\left(\right.$ mtaylor $\left(\right.$ expand $\left.\left.\left(v_{19} \cdot e_{12}\right), x, 64\right) \bmod 2\right) \#=v_{4}^{3} v_{8} v_{12} v_{19}=v_{4}^{-1} v_{8} v_{12} v_{19}$

$\operatorname{sort}\left(\right.$ mtaylor $\left.\left(\operatorname{expand}\left(v_{20} \cdot e_{12} \cdot e_{13}\right), x, 64\right) \bmod 2\right) \#=v_{1}^{11} v_{4}^{2} v_{5}^{3} v_{8}^{4} v_{9} v_{12}^{2} v_{13} v_{20}=v_{1}^{-5} v_{4}^{2} v_{5}^{3} v_{9}$ $v_{12}^{2} v_{13} v_{20}$

(169)

$\operatorname{sort}\left(\right.$ mtaylor $\left.\left(\operatorname{expand}\left(v_{21} \cdot e_{12} \cdot e_{6}^{2} \cdot e_{14}\right), x, 64\right) \bmod 2\right) \#=v_{2} v_{6}^{5} v_{10} v_{12}^{2} v_{14} v_{21}=v_{2} v_{6}^{-3} v_{10} v_{12}^{2} v_{14} v_{21}$

$\operatorname{sort}\left(\right.$ mtaylor $\left.\left(\operatorname{expand}\left(v_{22} \cdot e_{12} \cdot e_{6}^{2} \cdot e_{15}\right), x, 64\right) \bmod 2\right) \#=v_{1}^{13} v_{2}^{3} v_{3}^{3} v_{4}^{2} v_{5}^{7} v_{6} v_{7}^{3} v_{9}^{3}$

$v_{10}^{3} v_{11} v_{13} v_{14} v_{15} v_{22}=v_{1}^{-3} v_{2}^{3} v_{3}^{3} v_{4}^{2} v_{5}^{7} v_{6} v_{7}^{3} v_{9}^{3} v_{10}^{3} v_{11} v_{13} v_{14} v_{15} v_{22}$

$\operatorname{sort}\left(\right.$ mtaylor $\left.\left(\operatorname{expand}\left(v_{23} \cdot e_{5}^{2} \cdot e_{11} \cdot e_{6}^{2} \cdot e_{13} \cdot e_{3}^{4}\right), x, 64\right) \bmod 2\right) \#=v_{2}^{5} v_{3}^{7} v_{4}^{3} v_{5}^{5} v_{6}^{2} v_{9}^{2} v_{10} v_{11} v_{12} v_{13} v_{23}=$ $v_{2}^{5} v_{3}^{7} v_{4}^{-1} v_{5}^{5} v_{6}^{2} v_{9}^{2} v_{10} v_{11} v_{12} v_{13} v_{23}$

$\operatorname{sort}\left(\right.$ mtaylor $\left(\right.$ expand $\left.\left.\left(v_{24} \cdot e_{10} \cdot e_{12} \cdot e_{6}^{2} \cdot e_{14}\right), x, 64\right) \bmod 2\right) \#=v_{6}^{5} v_{8} v_{10}^{2} v_{12}^{2} v_{14} v_{24}$

$\operatorname{sort}\left(\right.$ mtaylor $\left.\left(\operatorname{expand}\left(v_{25} \cdot e_{11} \cdot e_{1}^{8} \cdot e_{6}^{2} \cdot e_{14} \cdot e_{7}^{2}\right), x, 64\right) \bmod 2\right) \#=v_{1} v_{2}^{2} v_{3}^{9} v_{4}^{3} v_{5}^{2} v_{6}^{7} v_{7}^{2} v_{9}$

$v_{10}^{2} v_{11} v_{12} v_{14} v_{25}=v_{1} v_{2}^{2} v_{3}^{-7} v_{4}^{-1} v_{5}^{2} v_{6}^{-2} v_{7}^{2} v_{9} v_{10}^{2} v_{11} v_{12} v_{14} v_{25}$

$\operatorname{sort}\left(\right.$ mtaylor $\left.\left(\operatorname{expand}\left(v_{26} \cdot e_{9} \cdot e_{10} \cdot e_{1}^{8} \cdot e_{12} \cdot e_{14}\right), x, 64\right) \bmod 2\right) \#=v_{1}^{15} v_{2}^{2} v_{4}^{2} v_{6}^{3} v_{8}^{6} v_{9} v_{10}^{2} v_{12}^{2} v_{14} v_{26}=$ $v_{1}^{-1} v_{2}^{2} v_{4}^{2} v_{6}^{3} v_{9} v_{10}^{2} v_{12}^{2} v_{14} v_{26}$

$\operatorname{Im}\left(f_{1}\right)=\left\langle h, v_{1}, v_{2}, \ldots, v_{11}\right\rangle=\langle h\rangle \times\left\langle e_{1}\right\rangle \times\left\langle e_{2}\right\rangle \times \ldots \times\left\langle e_{15}\right\rangle$ 


\section{Referências Bibliográficas}

[1] R. Z. Aleev, Higman's Central Unit Theorem, Units of Integral Group Rings and Fibonacci Numbers, Int. J. of Alg. and Comp. 4, 3 (1994), 309-358.

[2] R. Z. Allev and Z. Panina, The Units of Cyclic Groups of Orders 7 and 9, Russ. Math. 43, (2000), 80-83.

[3] H. Bass, Algebraic K-Theory, W. A. Benjamin, Inc, New York, (1968).

[4] S. D. Berman and T. Zh. Mollov, On Group Rings of Abelian p-Groups of Any Cardinality, Mat. Zametki 6 (1969), 381-392.

[5] A. Bovdi and A. A. Sakach, The unitary subgroup of the multiplicative group of the modular group algebra of a finite abelian p-group. (Russian) Mat. Zametki 45 (1989), no. 6, 23-29, 110; translation in Math. Notes 45 (1989), no. 5-6, 445-450.

[6] A. Bovdi and A. Szakács, A basis for the unitary subgroup of the group of units in a finite commutative group algebra, Publ. Math. Debrecen 46 (1995), no. 1-2, 97-120.

[7] A. Bovdi and S. K. Sehgal, Unitary subgroup of integral group rings, Publicacions Matem., 36 (1992), $197-204$.

[8] A. Bovdi, The Group of Units of a Group Algebras of Characteristic p, Publ. Math. Debrecen, 52 (1998), $193-244$.

[9] A. Bovdi and C. Polcino Milies, Normal Subgroups of the Group of Units in Group Rings of Torsion Groups, Publ. Math. Debrecen, 59 (2001), 235-242.

[10] A. Bovdi and A. Szakács, Units of Commutative Group Algebra with Involution, Publ. Math. Debrecen, 69 (2006), 291-296.

[11] A. Cayley, On the Theory of Groups as Depending on the Symbolic Equation $\theta^{n}=1$, Phil. Mag. 7, (1854) 40-47.

[12] D. B. Coleman and D. S. Passman, Units in modular group rings, Proc. Am. Math. Soc. 25 (1970), $510-512$. 
[13] N. Endo, On The Unit Group of the Group Ring $\mathbb{Z}[G]$, Tokio J. Math,(2002), v. 25, No. 2, 335-351.

[14] R. A. Ferraz, Groups Generated by a Bass Cyclic Unit and a Bicyclic Unit in the Units of Z[G]. Journal of Group Theory (2004), v. 7, p. 421-430.

[15] R. A. Ferraz, Units of $\mathbb{Z} C_{p}$, Groups, Rings and Group Rings, Contemp. Math. - Amer. Math. Soc., Providence, RI, (2009), v. 499, 107-119.

[16] A. Giambruno and S. K. Sehgal, Generators of Large Subgroups of Units of Integral Group Rings of Nilpotent Groups, Jornal of Algebra, 174 (1995), 150-156.

[17] J. Z. Gonçalves and P. M. Veloso, Special Units, Unipotent Units and Free Groups in Group Algebras. Contemp. Math. - Amer. Math. Soc., (Print) (2009), v. 499, p. 127-140.

[18] J. Z. Gonçalves and D. S. Passam, Unitary Units in Group Algebras, Israel Journal of Mathematics (2001), v. 125, p. $131-155$.

[19] J. Z. Gonçalves and D. S. Passman, Free Unit Groups in Group Algebras, Journal of Algebra (2001), v. 246, p. 226-252.

[20] J. Z. Gonçalves and A. del Rio, Bicyclic Units, Bass Cyclic Units and Free Groups, Journal of Group Theory (2008), v. 11, p. 247-265.

[21] G. J. Janusz, Faithful Representations of p-groups at Characteristic p, I. J. Algebra 15 (1970) 335-351.

[22] G. Higman, Units of Group Rings, Proc. London Math. Soc. 46 (1971), 205-214.

[23] E. Jespers, Free Normal Complements and the Unit Group of Integral Group Rings, 122 (1994), 59-66.

[24] E. Jerpers, Biclyclic Unit in Some Integral Group Rings, Canad. Math. Bull. 38 (1995), 80-86.

[25] E. Jespers and G. Leal, Generators of Large Subgroups of the Unit Group of Integral Group Rings, Manuscripta Math. 78 (1993), 303-315.

[26] E. Jespers and C. Polcino Milies, Units of Group Rings, J. Pure Appl. Algebra 107 (1996), 233-251.

[27] G. Karpilovsky, Commutative Group Algebras, Marcel Dekker, Inc. (1983).

[28] G. Karpilovsky, Unit Groups of Classical Rings, Oxford University Press, (1988).

[29] G. Karpilovsky, Unit Groups of Group Rings, Longman Scientific \& Technical, (1989).

[30] J. Kurdics, On the group algebras with metabelian unit groups, Periodica Math. Hungarica 32 (1966), 57-64.

[31] S. Lang, Algebra, Addison-Wesley Publishing Company, (1027). 
[32] P. A. Martin, Introdução à Teoria dos Grupos e à Teoria de Galois, Publicações do Instituto de Matemática e Estatística da Universidade de São Paulo, (1998).

[33] I. Niven, H. S. Zuckerman and H. L. Montgomery, The Theory of Numbers, Jonh Wiley \& Sons, Inc. (1991).

[34] M. M. Parmenter, Units and Indecomposability of Ideals in Group Rings, Quest. Math. 8 (1985), 2-14.

[35] M. M. Parmenter, Torsion Free Normal Complements in the Unit Groups of Integral Group Rings, C. R. Math. Rep. Acad. Sci. Canada, 12 (1990), 113-118.

[36] M. M. Parmenter and C. Polcino Milies, Groups Rings whose Units Form a Nilpontent or FC Group, Proc. Amer. Math. Soc. 68, 2 (1978), 247-248.

[37] C. Polcino Milies, The Units of the Integral Group Ring $\mathbb{Z} D_{4}$, Bol. Soc. Brasileira de Mat. 4 (1972), 85-82.

[38] C. Polcino Milies, Integral Group Rings with Nilpotent Unit Groups, Canad. J. Math. 28 (1976), 954-960.

[39] C. Polcino Milies and S. K. Sehgal, An Introduction to Group Rings, Kluwer Academic Publishers, (2002).

[40] C. Polcino Milies and S. K. Sehgal, Central Units of Integral Group Rings, Comm. Algebra 27 (1999), 6233-6241.

[41] J. Ritter and S. K. Sehgal, Integral Group Rings with Trivial Central Units, Proc. Amer. Math. Soc. 108 (1990), 327-329.

[42] J. Ritter and S. K. Sehgal, Construction of Units in Integral Group Rings of Finite Nilpotent Groups, Trans. Amer. Math. Soc. 324 (1991), 602-621.

[43] J. Ritter and S. K. Sehgal, Construction of Units in Integral Group Rings of Monomial ans Symmetric Groups, J. Algebra 142 (1991), 511-526.

[44] S. K. Sehgal, Topics in Group Rings, Marcel Dekker, (1978).

[45] S. K. Sehgal, Units in Integral Group Rings, Longman Scientific \& Technical, (1993).

[46] S. K. Sehgal and H. J. Zassenhaus, Integral Group Rings with Nilpotent Unit Groups, Comm. Algebra 12 (1977), $101-111$.

[47] A. Shalev. Metabelian unit groups of group algebras are usually abelian, J. Pure Appl. Algebra 72 (1991), 295-302.

[48] L. C. Washington, Introduction to Cyclotomic Fields, Springer-Verlag (1997). 